[B] Lawrence Berkeley Laboratory

UNIVERSITY OF CALIFORNIA

CHEMICAL SCIENCES DIVISION

$361: 1 " 10$

\title{
Threshold Photodetachment Spectroscopy of Negative Ions
}

T.N. Kitsopoulos

(Ph.D. Thesis)

December 1991

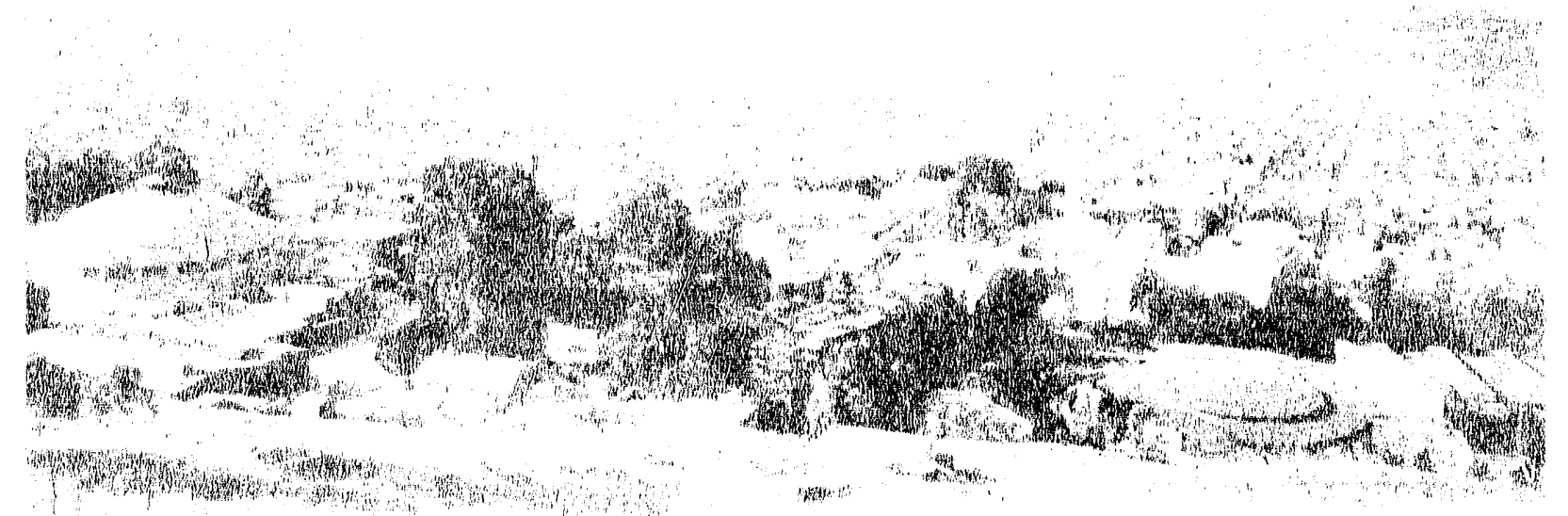

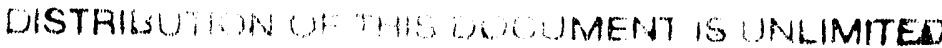

Prepared for the U.S. Department of Energy under Contract Number DE-A C03-76SF00098 


\section{DISCL.AIMER}

This document was prepared as an account of work sponsoned by the United States Government. Neither the United States Government nor any agency thereot, nor the Regents of the liniversity of California, nor any of their employees, makes any warrarty, express or implied, or assumes any legal liability or responsibility tor the accuracy, completeness, or usefulnes of any information, apparatus, product, or process disclosed, or represents that its use would not infringe privately owned rights. Reference herein to any specific commercial product, process, or service by its trade name, srademark, manufacturer, or otherwise, does not necessarily constitute or imply its en. dorsement, recommendation, or favoring by the Unifed States Covemment or any gency thereor, or The Regents of the University of Caifornia. The views and opinons of authors expressed herein do not necessarily state or reflect those of the United States Government or any agency thereot or The Regents of the University of California and shall not be used for advertising or product endorsement purposes.

Lawrence Berkeley Laboratory is an equal opportunity employer.

This report has been reproduced directly from the best available copy. 
LBL -3.1876

DE92 017090

\title{
Threshold Photodetachment Spectroscopy of Negative Ions
}

\author{
Theofanis N. Kitsopoulos \\ Ph.D. Thesis \\ Department of Chemistry \\ University of California \\ and \\ Chemical Sciences Division \\ Lawrence Berkeley Laboratory \\ University of California \\ Berkeley, CA 94720
}

December 1991

This report has been reproduced directly from the best available copy.

This work was supported in part by the Office of Naval Research under Grant No. N0014-87-K-(0495, and by the Director, Office of Energy Research, Office of Basic Energy Sciences, Chemical Sciences Division, of the U.S. Department of Energy under Contract No. DE-AC03-76SF00098. 


\title{
Threshold Photodetachment Spectroscopy of Negative lons
}

\author{
Copyright $\odot 1991$
}

by

\author{
Theofanis N. Kitsopoulos
}

\section{DISCLAIMER}

\begin{abstract}
This report was prepared as an account of work sponsored by an agency of tle United States Government. Neither the United States Government nor any agency thercof, , or any of their employces, makes any warranty, express or implied, or assumes any legal liabil ty or responsibility for the accuracy, completeness, or usefulness of any information, apparatus, product, or process disclosed, or represents that its use would not infringe privately owned rights. Reference herein to any specific commercial product, process, or service by trade name, trademark, manufacturer, or otherwise does not necessarily constitute or imply its endorsement, recommendation, of favoring by the United States Government or any agency thereof. The views and opinions of authors expressed herein do not necessarily state or retlect those of the United States Government or any agency thereof.
\end{abstract}

The U.S. Department of Energy has the right to use this thesis for any purpose whatsoever including the right to reproduce all or any part there of 


\title{
Threshold Photodetachment Spectroscopy \\ of Clusters and Radicals
}

by

\section{Theofanis N. Kitsopoulos}

\begin{abstract}
This thesis is concerned with the development and application of high resolution threshold photodetachment spectroscopy of negative ions. Chapter I deals with the principles of our photodetachment technique, and in chapter II a detailed description of the apparatus is presented. The threshold photodetachment spectra of $\mathrm{I}^{-}$, and $\mathrm{SH}^{-}$, presented in the last sections of chapter II, indicated that a resolution of $3 \mathrm{~cm}^{-1}$ can be achieved using our technique. In chapter III the threshold photodetachment spectroscopy study of the transition state region for the $\mathrm{I}+\mathrm{HI}$ and I + DI reactions is discussed. Our technique probes the transition state region directly, and the results of our study are the first unambiguous observations of reactive resonances in a chernical reaction. Chapters IV, V and VI are concerned with the spectroscopy of small silicon arid carbon clusters. From our spectra we were able to assign electronic state energies and vibrational frequencies for the low lying electronic states of $\mathrm{Si}_{n}(n=2,3,4), \mathrm{C}_{5}$ and their corresponding anions.
\end{abstract}


Table of Contents

Abstract $\ldots \ldots \ldots \ldots \ldots \ldots \ldots \ldots \ldots \ldots \ldots \ldots \ldots \ldots \ldots$

Dedication $\ldots \ldots \ldots \ldots \ldots \ldots \ldots \ldots \ldots \ldots \ldots \ldots \ldots$

Acknowledgements $\ldots \ldots \ldots \ldots \ldots \ldots \ldots \ldots \ldots \ldots \ldots$ vi

Chapter I. Threshold Photodetachment Spectroscopy

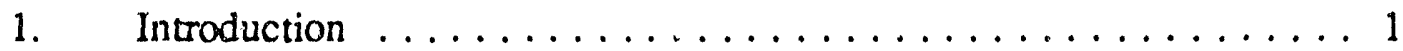

2. ZEKE Detection $\ldots \ldots \ldots \ldots \ldots \ldots \ldots \ldots$

3. Space Charge $\ldots \ldots \ldots \ldots \ldots \ldots \ldots \ldots \ldots$

4. Wigner's Law ...................... 14

Figure Captions $\ldots \ldots \ldots \ldots \ldots \ldots \ldots \ldots \ldots \ldots \ldots$

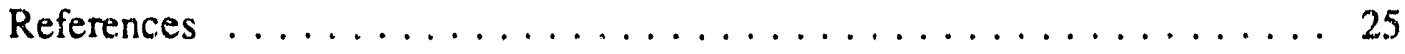

Chapter II. A Negative Ion

Threshold Photodetachment Spectrometer

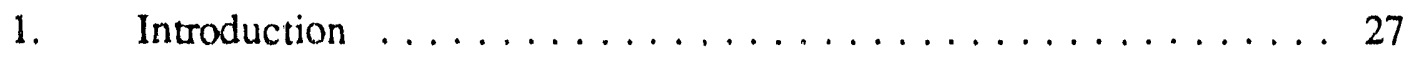

2. Source Region $\ldots \ldots \ldots \ldots \ldots \ldots \ldots \ldots$

a. Molecular Beam . . . . . . . . . . . . . . 28

b. Electron Gun/Negative Ion Production ............ . 34

c. Floating Scheme $\ldots \ldots \ldots \ldots \ldots \ldots \ldots$

d. Laser Vaporization Source . . . . . . . . . . . . 38

3. Mass Spectrometer ..................... 44

a. Ion Acceleration $\ldots \ldots \ldots \ldots \ldots \ldots \ldots$

b. Beam Modulation Time of Flight (BMTOF) . . . . . . . 46 
4. Detector Region $\ldots \ldots \ldots \ldots \ldots \ldots \ldots \ldots \ldots \ldots, 53$

5. Timing and Data Acquisition $\ldots \ldots \ldots \ldots \ldots \ldots \ldots \ldots$

6. Vacuum Interlock System $\ldots \ldots \ldots \ldots \ldots \ldots \ldots \ldots \ldots$

7. Photodetachment of $\mathrm{I}^{-} \ldots \ldots \ldots \ldots \ldots \ldots \ldots \ldots \ldots . \ldots \ldots$

8. Photodetachment of $\mathrm{SH}^{-} \ldots \ldots \ldots \ldots \ldots \ldots \ldots \ldots \ldots$

9. Conclusion $\ldots \ldots \ldots \ldots \ldots \ldots \ldots \ldots \ldots \ldots \ldots \ldots \ldots \ldots \ldots$

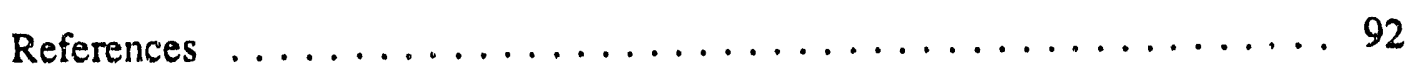

Chapter III. Threshold photodetachment study

of the transition state region for the

$$
\mathrm{I}+\mathrm{HI} \text { and } \mathrm{I}+\mathrm{DI} \text { reactions }
$$

1. Introduction $\ldots \ldots \ldots \ldots \ldots \ldots \ldots \ldots \ldots \ldots \ldots$

2. Experimental ..................... 94

3. Results and Discussion $\ldots \ldots \ldots \ldots \ldots \ldots \ldots \ldots . \ldots 5$

a. Photodetachment of $\mathrm{IHI}^{-} \ldots \ldots \ldots \ldots \ldots \ldots \ldots$

b. Photodetachment of $\mathrm{IDI}^{-} \ldots \ldots \ldots \ldots \ldots \ldots \ldots$

4. Conclusion $\ldots \ldots \ldots \ldots \ldots \ldots \ldots \ldots \ldots \ldots \ldots \ldots$

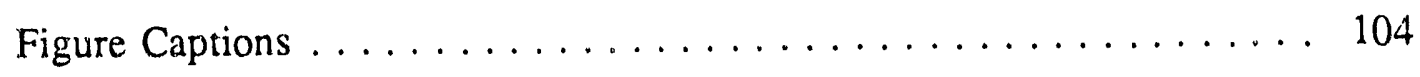

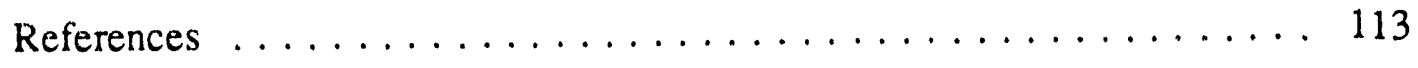

Chapter IV. Study of the low-lying electronic states of $\mathrm{Si}_{2}$

and $\mathrm{Si}_{2}-$ using negative ion photodetachment techniques

1. Introduction $\ldots \ldots \ldots \ldots \ldots \ldots \ldots \ldots \ldots \ldots \ldots \ldots$

2. Experimental $\ldots \ldots \ldots \ldots \ldots \ldots \ldots \ldots \ldots \ldots \ldots \ldots \ldots$ 


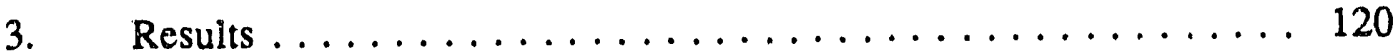

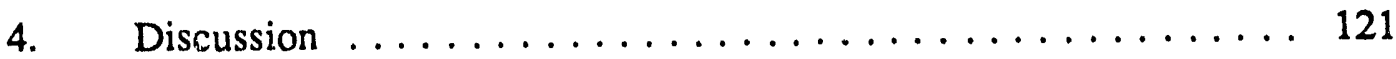

5. Conclusion $\ldots \ldots \ldots \ldots \ldots \ldots \ldots \ldots \ldots \ldots$

Figure Captions $\ldots \ldots \ldots \ldots \ldots \ldots \ldots \ldots \ldots \ldots \ldots \ldots \ldots \ldots$

Table Captions . . . . . . . . . . . . . . . . . . . . 133

References $\ldots \ldots \ldots \ldots \ldots \ldots \ldots \ldots \ldots \ldots \ldots \ldots \ldots$

Chapter V. Vibrationally resolved spectra of $\mathrm{Si}_{3}{ }^{-}$and $\mathrm{Si}_{4}{ }^{-}$

1. Introduction $\ldots \ldots \ldots \ldots \ldots \ldots \ldots \ldots \ldots$

2. Experimental $\ldots \ldots \ldots \ldots \ldots \ldots \ldots \ldots \ldots$

3. Results and Discussion . . . . . . . . . . . . . . . 150

4. Conclusion $\ldots \ldots \ldots \ldots \ldots \ldots \ldots \ldots \ldots \ldots$

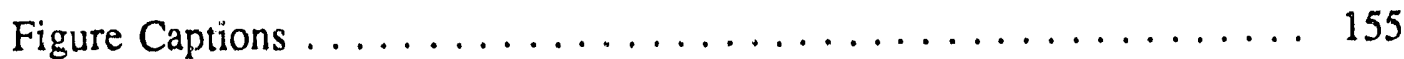

Table Captions . . . . . . . . . . . . . . . . . . . . 155

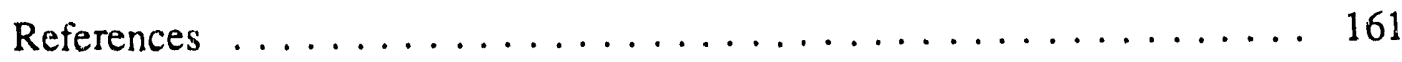

Chapter VI. Threshold Photodetachment Spectroscopy of $\mathrm{C}_{5}{ }^{-}$

1. Introduction $\ldots \ldots \ldots \ldots \ldots \ldots \ldots \ldots \ldots \ldots \ldots$

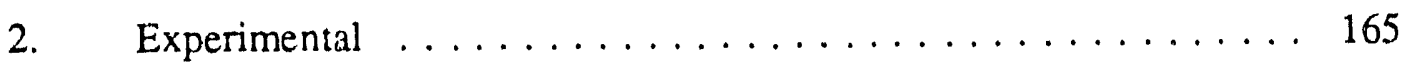

3. Results and Discussion . . . . . . . . . . . . . . 165

4. Conclusion $\ldots \ldots \ldots \ldots \ldots \ldots \ldots \ldots$

Figure Caption $\ldots \ldots \ldots \ldots \ldots \ldots \ldots \ldots \ldots \ldots \ldots \ldots \ldots$

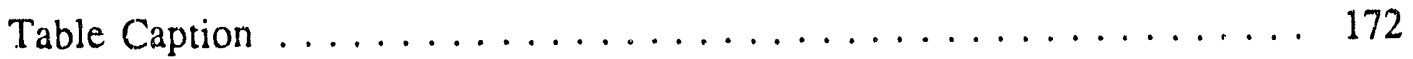

References $\ldots \ldots \ldots \ldots \ldots \ldots \ldots \ldots \ldots \ldots \ldots$ 
Dedicated to my parents

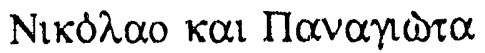




\section{Acknowledgments}

For the last five years I have been involved in some of the most interesting research projects in physical chemistry. I had the opportunity to work with some of the smartest people and learn from their wisdom and experience. I would like to thank my research advisor Prof. Dan Neumark for five years of scientific guidance and stimulation. I was fortunate enough to join the Neumark research team during its conception, and be a participant in its evolution into one of the "hottest", most intense, interesting and "fun to be in" groups in physical chemisiry. I would like to thank Dan for entrusting me with the construction and operation of a couple of state the art photoeiectron spectrometers. I would like to thank him for always showing both respect and support for my ideas.

Although Alex Weaver and I got off on the wrong foot, over the last five years we have become two very close friends. Our views differ on pretty much every aspect of life. We do agree on the laws of physics, but even then our approaches are completely out of phase. Alex keeps reminding me that there are alternative ways to certain things and I need not be so stubborn and hardheaded. The worse thing is that she might have actually convinced me of this. Thank you Alex.

I would like to thank Ricky Metz who has been the computer "guru" of our group from day one. I have sought his expertise on numerous occasions, and he has never failed me once. One amazing thing about Ricky is that he never seems to get very upset about almost anything, which to a hot tempered guy like me is beyond comprehension.

I would like to thank Jenny Loeser and Prof. Irene Waller (Univ. of British Columbia) for their help during the construction of the threshold spectrometer. The first 
two years of the construction of the "ACROPOLIS", were very difficult since we were developing some new technology and were uncertain about the outcome. As if this wasn't enough Jenny and Irene had to put up with my occasional bursts of temper, directed never to them but to the machine, and I know how ugly I can get on certain occasions. I admired Irene's forward "beyond b...h" character which made it easy for the two of us to communicate. Jenny is responsible for writing all the software which runs the experiment; to give an honest estimate, I think it would have taken me over a year to develop this myself. Jenny also lead the way in the construction and characterization of the original pulsed valve driver, and several data acquisition systems. She was always thorough (sometimes too much) in her work, and always provided excellent documentation for the future users.

A special thanks to two very good friends and colleagues Dr. Bob Continetti and Doug Cyr, for numerous discussions on subject matters that covered almost every topic both scientific and non-scientific. Any new idea, any controversial issue or any worthwhile observation I would make, I would immediately relay it to Doug or Bob, because somehow I knew they would be just as excited as I was. Bob's enthusiasm for "good science", and his "gung-ho" approach has inspired me through my graduate career. I greatly regret the fact the I was not able to collaborate directly with either the Dougmeister or the Bob-man on a particular experiment, and perhaps this is why I find myself hanging around their lab more than I do around my former one.

Steve Bradforth my fellow European, probably the only person in the group more stubborn than me, many thanks for numerous heated debates and discussions, which I 
don't think I ever won, but then again I probably won't admit losing any of them either. I also thank Don Arnold for improving the quality of our softball team and assistance with the Franck-Condon simulation program. To Caroline Chick, my colleague and trainee for the last two years, thanks and good luck. Thanks also to Yuexing Zhao and Eun-Ha Kim.

In building a new apparatus there is a great deal of mechanical design and construction involved. I thank George Weber (now Head of Physics Machine Shop) for many helpful suggestions on some of my designs and especially for the tremendously fast turn around time on almost all my jobs. A would also like to thank Ron Dal-Porto, Andy Anderson and machinists Larry Hull, Eric Granlund, Hans Graetsch, Dave Murai, Scott Bonilla and Chuck Souders. Also thanks to Norman Tom, Bob Dunn, Bob Koch, Carl Lamey, Mike Murffey and Brenda Jefferson. I would also like to thank the late Bill Wilke of the LBL ceramic shop.

I would like to thank the Lee group and Saykally group from which I have been borrowing equipment for the last five years. A special thanks to Prof. Rich Saykally, and Prof. Doros Theodorou for advice, recommendation letters and taking time to read my thesis. I would also like to thank Prof. Eric Gislason (Univ. of Illinois at Chicago) and Prof. Robert Gordon (Uriv. of Illinois at Chicago) for getting me interested in physical chemistry.

Last but not least I would like to thank my closest friends Antoni, Soula, Doreta and Kouli (partner in crime) who have put up with me during these five years, and were there when I needed them the most. Thanks also to my former and present roommates 
Nike and Dimimi for tolerating my existence. Above all I would like to thank my parents for their love, trust and support, and enormous bet'lef in education which has kept me going through school all these years.

This research was supported in pax: by the Director, Office of Energy Research, Office of Basic Energy' Sciences, Chemical Sciences Division of the U.S. Department of Energy under contract No. DE-AC03-76SF00098, and in part by the Office of Naval Research Grant No. N0014-87-K-0495. 


\section{Chapter I}

\section{Threshold Photodetachment Spectroscopy}

\section{Introduction}

Since the 1905 explanation of the photoelectric effect by Einstein, ${ }^{1}$ scientists have used it in all sorts of applications. One such application has been photoelectron spectroscopy (PES) which, over the years, has yielded an abundance of spectroscopic information for many atomic and moiecuiar systems. More recently, photoelectron spectroscopy of negative ions has emerged as a versatile and very powerful technique for the studies of a variety of exotic chemical species. These species include reactive free radicals, ${ }^{2}$ size selected clusters, ${ }^{3}$ and unstable collision complexes formed in chemical reactions. ${ }^{4}$ Photodetachment of riegative ions is appealing because it allows for mass selection prior to spectroscopic interrogation, thus eliminating any uncertainty surrounding the identity of the signal carrier. In a typical photoelectron spectioscopy experiment, a mass selected beam of negative ions is photodetached using the output of an appropriate munoihromatic light source, and the ejected photoelectrons are collected and energy analyzed. Spectroscopic information for both the anion precursor and the neutral product can obtained by these types of experiments. In addition, selection rules for photoelectron spectroscopy enable us to probe electronic states which are inaccessible to more conventional optical spectroscopic methods. ${ }^{5.6}$

Although a wide selection of electron energy analyzers are available to the photoelectron spectroscopist, rarely do they offer a resolution better than about $5 \mathrm{meV}$ (40 
$\left.\mathrm{cm}^{-1}\right) .^{7.8}$ Recently, Müller-Dethlefs and Schlag ${ }^{9}$ have developed a new photoelectron technique which employs a clever zero kinetic energy (ZEKE) electron analyzer, and offers resolution on the order of $1 \mathrm{~cm}^{-1}$. Neumark and coworkers ${ }^{10}$ have successfully applied a similar detection scheme to the photodetachment of negative ions, and have achieved a resolution of $3 \mathrm{~cm}^{-1}$. At this resolution, vibrational progressions in even the floppiest molecules can be resolved, thus addressing questions surrounding the inhomogeneity of brcad peaks which typically appear in various photoelectron spectra. As will be discussed in the sections that below, ZEKE photodetachment spectroscopy of a fast inn beam is constrained by the non-zero center of mass velocity (in the lab frame), which is not a factor in the photoionization of nearly stationary neutral molecules.

\section{ZEKE Detection}

In the ZEKE detection scheme, a tunable light source is used to photodetach the negative ion and only photoelectrons with near zero kinetic energy are then detected as a function of photon energy. This is accomplished by the introduction of a small time delay after photodetachment which allows energetic electrons to spatially separate from the ZEKE electrons; to extract the electrons, a weak pulsed field is then applied and, due to the initial separation, between the ZEKE and energetic electrons, the two sets of electrons will gain different energies in the electric field and will arrive at the detector at different times. This allows us to use gated detection in order to count only those electrons arriving at the detector at times appropriate for ZEKE electrons.

In this section, we will explain how the spatial and temporal separations for the various photoelectrons is achieved, using simple kinematic considerations. Consider a 
bunch of negative ions of mass $m_{i 0 n}$, moving along the $x$-axis of Fig.1 with laboratory energy $E_{i o n}$. At some point along the axis, the ion bunch is intersected by a monochromatic photon beam from a turable light source. If $E_{b b}$ is defined as the minimum energy (threshold) required to detach an electron, and the photon energy is hv, then the energy of an ejected photoelectron is given by the equation :

$$
E_{a}(C M)=h v-E_{t h}
$$

where $E_{e}(C M)$ refers to the center of mass $(C M)$ kinetic energy of the photoelectron. Shown in Fig. 1 are $\underline{v}_{-}(\mathrm{CM})$, the photoelectron velocity in the center of mass frame, and $\underline{V}_{C M}$, the center of mass velocity for the system photoelectron-neutral, which coincides with the anion beam velocity in the laboratory frame. After photodetachment has occurred, the laboratory velocity $\underline{v}_{e}(\mathrm{Lab})$ of the ejected photoelectron is given by the vector sum :

$$
v_{e}(L a b)=y_{e}(C M)+Y_{C M}
$$

which we can rewrite as

$$
E_{a}(I a b)=E_{a}(C M)+E_{\text {lon }} \cdot \frac{m_{e}}{m_{\text {ion }}}+2 \cdot \sqrt{E_{e}(C M) \cdot E_{\text {ion }} \frac{m_{e}}{m_{\text {ion }}}} \cdot \cos \theta
$$

where $E_{e}(L a b)$ and $m_{e}$ are the laboratory kinetic energy and mass of the photoelectron, and $\theta$ is the angle between the $\underline{v}_{e}(\mathrm{CM})$ and $\underline{\mathrm{V}}_{\mathrm{CM}}$.

Let $\tau$ be the time delay introduced after the photodetachment of the ion packet. Although photoelectrons are ejected in a $4 \pi$ solid angle, due to the azimuthal symmetry of the process with respect to the anion beam direction, we will only calculate the final 
positions of the various photoelectrons in the xy plane. These are given by the equations

$$
y=\left|x_{e}(C M)\right| \cdot \tau \cdot \sin \theta=\sqrt{\frac{2 E_{e}(C M)}{m_{e}}} \cdot \tau \cdot \sin \theta
$$

and

$$
x=\left(\left|y_{e}(C M)\right| \cdot \cos \theta+\left|Y_{C M}\right|\right) \cdot \tau=\left(\sqrt{\frac{2 E_{e}(C M)}{m_{e}}} \cdot \cos \theta+\sqrt{\frac{2 E_{\text {lon }}}{m_{\text {bon }}}}\right) \cdot \tau
$$

where $x$ and $y$ are the distance travelled parallel and perpendicular to the $x$-axis. For ZEKE electrons for which $\underline{v}_{e}(C M) \rightarrow 0$, eq. 4 implies that $y(Z E K E) \rightarrow 0$, thus all the ZEKE photoelectrons will follow paraxial trajectories during the delay $\tau$; we define a paraxial trajectory as the one where $\alpha=0$, and $\alpha$ is the angie between $\underline{v}_{\ell}(\mathrm{Lab})$ and $\underline{\mathrm{V}}_{\mathrm{CM}}$. For an energetic electron with energy $E_{e}(C M)=15 \mathrm{~cm}^{-1}$, we have listed in the table below the various displacements y for a delay $\tau=500 \mathrm{nsec}$.

\begin{tabular}{|c|c|c|c|}
\hline$E_{e}(\mathrm{CM})$ & $\theta$ & $y(\mathrm{~cm})$ & $x(\mathrm{~cm})$ \\
\hline \multirow{1}{*}{$15 \mathrm{~cm}^{-1}$} & $30^{\circ}$ & 0.67 & 3.41 \\
\cline { 2 - 4 } & $90^{\circ}$ & 1.16 & 2.94 \\
\cline { 2 - 4 } & $120^{\circ}$ & 1.16 & 1.62 \\
\cline { 2 - 4 } & $150^{\circ}$ & 0.67 & 1.15 \\
\hline
\end{tabular}


In order to calculate the $\mathrm{x}$-displacements in the table above, we assumed an ion beam energy of $1 \mathrm{keV}$, and an ion mass of $100 \mathrm{amu}$. From the table it is clear that some of the energetic electrons will be displaced substantially off-axis. Hence we can now discriminate between the paraxial ZEKE and the off axis energetic electrons using an array of slits centered about the ion beam axis similar to the illustartion in Fig. 2.

As is obvious from Fig. 2, not all the energetic photoelectrons have been discriminated against by the slits introduced in the previous paragraph. There remain those energetic electrons, which like the ZEKE electrons, followed paraxial trajectories i.e. the backward $\left(\theta=180^{\circ}\right)$ and forward scattered electrons $\left(\theta=0^{\circ}\right)$. Rewriting eq. 5 in terms of the electron energies we obtain

$$
x=\tau \cdot\left(\sqrt{\frac{2 \cdot E_{\text {ton }}}{m_{i o n}}} \pm \sqrt{\left.\frac{2 E_{e}(C M)}{m_{e}}\right)}\right.
$$

where + and - refer to the forward and backward scattered electrons respectively. Since $E_{c}^{Z E K E}(C M) \rightarrow 0$ we rewrite eq. 6 to obtain the approximate expression

$$
|x(B K W D)-x(Z E K E)|=|x(F R W D)-x(Z E K E)|=\tau \cdot \sqrt{\frac{2 E_{e}(C M)}{m_{e}}}
$$

We note that the relative distances travelled are independent of $\underline{V}_{C M}$ which is intuitively correct since all the photoelectrons belong to the same center of mass frame, hence any motion within this frame is independent of the center of mass velocity. Separation of these electrons can now accomplished by applying a uniform electric field whose 
direction is parallel to $\underline{V}_{C M}$, as shown in Fig. 3. For illustration purposes we will only consider the forward (FRWD) scattered electrons while the same derivations presented bellow can be trivially applied to the backward scattered electrons.

Let $\mathrm{F}$ be the electric field, and $\mathrm{S}(\mathrm{ZEKE})$ and $\mathrm{S}$ (FRWD) the distances travelled by the ZEKE and FRWD scattered electrons in the electric field as shown in Fig. 3. Although $y(Z E K E) \rightarrow 0$, it is obvious from eq. 5 that $x(Z E K E)$ is non-zero. Actually for the $1 \mathrm{keV}$ and $100 \mathrm{amu}$ ion beam mentioned above $x($ ZEKE) $=2.28 \mathrm{~cm}$, for a delay of 500 nsec. Thus it is imperative that the extraction field of fig. 3 be an extendended field such that all the ZEKE electrons are extracted. Since $S(Z E K E) \geq S$ (FRWD), after some time $T_{f}$, the ZEKE electrons will gain more energy than the FRWD electrons and hence catch up with them at some point $X_{f}, t_{t}$ and $X_{t}$ are related by the equations :

$$
\varepsilon_{f}=\frac{\sqrt{2 m_{e}}}{e . F} \cdot\left(\left(\sqrt{U_{F R W D}+e . S(F R W D) \cdot F}-\sqrt{U_{F R W D}}\right)+\frac{X_{f} e . F}{2 \sqrt{U_{F R W D}+e . S(F R W D) \cdot F}}\right)
$$

and

$$
\left.t_{f}=\frac{\sqrt{2 m_{e}}}{e . F}, i\left(\sqrt{U_{Z E K E}+e . S(Z E K E) \cdot F}-\sqrt{U_{Z E K E}}\right)+\frac{X_{f} e . F}{2 \cdot \sqrt{U_{Z E K E}+e . S(Z E K E) \cdot F}}\right\}
$$

where $U_{\text {FRWD }}$ and $U_{\text {ZEKE }}$ are the initial lab kinetic energies of the FRWD and ZEKE photoelectrons before the field $\mathrm{F}$ is applied, and $\mathrm{F}$ is the magnitude of the electric field E. From equations 8 and 9 we can derive the expression for $X_{f}$ which is 


$$
\begin{gathered}
X_{f}=\frac{2 \cdot \sqrt{U_{Z E R E}+e . S(Z E K E) \cdot F} \cdot \sqrt{U_{F R H D}+e . S(F R W D) \cdot F}}{e . F} . \\
\left(1+\frac{\sqrt{U_{F R H D}}-\sqrt{U_{Z E R E}}}{\sqrt{U_{\mathrm{ZERE}}+e . S(Z E K E) \cdot \bar{F}}-\sqrt{U_{F R W D}+e . S(F R W D) \cdot F}}\right) .
\end{gathered}
$$

Since both $U_{\text {FRWD }}$ and $U_{\text {ZEKE }}$, whose values are typically about $10 \mathrm{meV}$, are much smaller than the e.S(ZEKE or FRWD).F energy term (that is, the energy the electrons acquire from the extraction field), equation 10 becomes:

$$
\begin{gathered}
X_{f}=2 \cdot \sqrt{S(Z E K E) \cdot S(F R W D)} \\
\text { (when } \left.U_{Z E K E}-0, U_{F R W D}-0\right)
\end{gathered}
$$

The distance at which the two electron packets focus is independent of the magnitude of the applied electric field, and is only dependent on the initial positions of the electron packets within the electric field. This situation is called geometric focusing, ${ }^{11}$ and occurs only when the initial velocities of the electron clouds are small relative to the velocities imparted by the extraction field. Similarly the geometric focusing point $X_{f}^{\prime}$ for the BKWD and ZEKE electrons is given by

$$
X_{f}^{\prime}=2 \sqrt{S(Z E K E) \cdot S(B K W D)}
$$

where S(BKWD) is the initial position of the BKWD electrons in the electric field.

Beyond the respective focus points, the separation of the ZEKE electrons from the FRWD and BWRD electrons will increase linearly as a function of time. Hence it is desirable to place the electron detector at a distance $L$ from the exit of the electric field, such that $L \geq X_{r}$ and $X_{r}^{\prime}$. In addition, to maximize the "spreading out time", one wants 
to minimize the $\mathrm{X}_{\mathrm{f}}{ }_{\mathrm{f}}$ focusing distance. This can be acheived by extracting the electrons when S(ZEKE) is very small i.e. when the threshold electrons are near the edge of the field. By doing this, one achieves excellent discrimination against the FRWD electrons which will be located outside the field during the extraction and thus not accelerated. The disadvantage is that near the edge, fringe fields become very pronounced and might influence the electron trajectories such that some electrons will not reach the detector, thus causing a decrease in the collection efficiency. Experimentally, we find that $\mathrm{S}(\mathrm{ZEKE})$ distances between 0.5 and $1 \mathrm{~cm}$ offer the best results when $\mathrm{L}=60 \mathrm{~cm}$. Finally, the photoelectrons can now be counted using a gated detection scheme, and collecting only electrons with the appropriate arrival times for threshold electrons.

In light of the previous discussion, it is clear that any stray electric or magnetic fields near the photodetachment region during the time delay $\tau$ will inhibit the proper function of the ZEKE analyzer. Two additional factors which limit the success of the negative ion photodetachment technique are: space charge (an experimental limitation), and Wigner's Law which is concerned with the photodetachment cross section near threshold and is a natural limitation.

\section{Space Charge}

Space charge is defined as the energy gained or lost by a photoelectron due to the influence of surrounding charged particles which, in our situation, are the precursor negative ions. Because the energy of the threshold photoelectrons is very small (about 3 to $10 \mathrm{~cm}^{-1}$ ), these electrons are especially vulnerable to space charge effects.

Lets consider the situation where $\mathrm{N}$ singly charged negative ions are confined in 
a sphere with radius $R$. If a photoelectron is generated in the center of such a cloud we can calculate the energy gained by the electron as it moves from the sphere center $(r=0)$ to the detector $(r=\infty)$. If we assume that the anions are uniformly spread within the sphere then the charge density of the sphere $Q$ can be approximated by

$$
\rho=-\frac{3 \cdot e \cdot N}{4 \cdot \pi \cdot R^{3}}
$$

and we can calculate the electric field inside the ion cloud using Gauss' law:

$$
\begin{gathered}
\int_{S} E \cdot d d=\frac{1}{\epsilon_{0}} \cdot \int_{V} \rho \cdot d V \\
E_{r}\left(4 \cdot \pi \cdot r^{2}\right)=-\frac{3 \cdot e \cdot N}{\epsilon_{0} \cdot 4 \cdot \pi \cdot R^{3}} \cdot\left(\frac{4}{3} \cdot \pi \cdot r^{3}\right) \\
E_{r}=-\frac{e \cdot N \cdot r}{\epsilon_{0} \cdot 4 \cdot \pi \cdot R^{3}}
\end{gathered}
$$

where $S$ and $V$ represent the surface and volume of a sphere of radius $r$, which is concentric with the ion cloud, $E$ is the electric field at the surface of this sphere, and $E_{r}$ is its magnitude in the radial direction.

If $\phi_{\text {in }}$ is the electric potential inside the ion sphere then

$$
\begin{aligned}
\nabla \phi_{i n}=-E & \Rightarrow \int \nabla \phi_{i n} \cdot d r=-\int E \cdot d L=-\int_{0}^{R} E_{r} \cdot d r \Rightarrow \\
& \phi_{i n}(R)-\phi_{i n}(0)=\frac{e \cdot N}{8 \cdot \pi \cdot \varepsilon_{0} \cdot R}
\end{aligned}
$$

Hence the work $W_{\text {in }}$ necessary to move the electron from the center of the anion cloud to the surface is given by 


$$
W_{L n}=-e \cdot\left(\phi_{L n}(R)-\phi_{L n}(0)\right)=-\frac{e^{2} \cdot N}{8 \cdot \pi \cdot \varepsilon_{0} \cdot R} .
$$

In addition the work needed to move the electron from surface of the sphere to infinity is given by

$$
\begin{gathered}
W_{\text {out }}=-e .(\phi(\infty)-\phi(R))=-\frac{e^{2} \cdot N}{4 \cdot \pi \cdot \epsilon_{0} \cdot R} \\
\text { where } \phi(\infty) \rightarrow 0 \text { and } \phi(R)=-\frac{e \cdot N}{4 \cdot \pi \cdot \varepsilon_{0} \cdot R} .
\end{gathered}
$$

The total work done by the photoelectron due to space charge now becomes

$$
W_{\text {tot }}=W_{\text {in }}+W_{\text {out }}
$$

which upon substitution yields

$$
W_{t o t}=-\frac{3 \cdot e^{2} \cdot N}{8 \cdot \pi \cdot \varepsilon_{0} \cdot R}
$$

and the negative sign indicates that the photoelectron gains energy due to the space charge effect. Hence the final energy of the electron $E_{\text {final }}$ is given by

$$
E_{\text {final }}=E_{e}(C M)-W_{\text {tor }}
$$

If the threshold electrons gain sufficient energy they will no longer be ZEKE electrons and will be discriminated against by virtue of our detection scheme. Thus, although in conventional photoelectron spectroscopy, space charge results only in an energy shift of the spectrum transitions, in ZEKE spectroscopy space charge will actually cause a decrease in the observed signal. To quantify the space charge limits, consider $\mathrm{N}=1000$ ions within an interaction sphere of $\mathrm{R}=2 \mathrm{~mm}$; for this situation using eq. 20 we 
find that $W_{t o t}=-9 \mathrm{~cm}^{-1}$. If we assume that $E_{e}(C M)=0$, then the the final energy of the electron is $E_{\text {[inal }}=9 \mathrm{~cm}^{-1}$. Thus if the electron detector is gated such that it collects only electrons with $E_{e} \leq 5 \mathrm{~cm}^{-1}$, then any threshold electrons generated in the situation just described will not be detected. Furthermore, due to the finite spatial extent of the photodetachment laser, ZEKE electrons will be produced at various positions within the ion cloud. Since the energy gained by the individual electrons due to space charge will depend on its initial position, this results in an energy spread of the ZEKE cloud. It is thus obvious now that increa.ng the amount of ions in the interaction region does not necessarily produce more ZEKE electrons.

There are two ideal situations which minimize space charge effects : one where the ion packet consists of a single ion and the other where the photon flux is sufficient to photodetach nearly all of the initial anions. The former situation is experimentally a near impossibility since mass selecting single particles is far from routine; as will be mentioned in the experimental section, we typically mass select an ion bunch containing $10^{3}$ to $10^{4}$ ions. The second situation is not really a space charge free condition because if one starts with $10^{3}$ anions and photodetaches all of them, the $10^{3}$ electrons produced within the same volume as the anion cloud, will cause a "secondary" space charge effect, due to the repulsion between the photoelectrons. However, since all the electrons posses very low energies, the net effect will be a spatial divergence of the electron cloud rather than severe energy gains such as those described by eq. 20 ; this type of divergence can be corrected trivially using an electrostatic einzel lens (see experimental section).

To determine if complete neutralization of an ion cloud is possible, consider the 
relationship between the phenomenological photodetachment cross section $\sigma_{d}$, and the photon absorption, which is given by Beer's Law :

$$
N=N_{0} \cdot \exp \left(-\sigma_{d} \frac{n_{\text {lons }}}{V} x\right)
$$

where $N_{0}$ and $N$ are the initial and final number of photons entering and leaving an interaction volume containing $n_{\text {ions }}$ number of ions, $V$ and $x$ are the volume and length of the interaction region. Rewriting eq. 21 we find

$$
\begin{gathered}
\ln (N)=\ln \left(N_{0} \cdot \exp \left(-\sigma_{d} \frac{n_{\text {ions }}}{V} x\right)=\ln \left(N_{0}\right)-\sigma_{d} \frac{n_{\text {ions }}}{V} x-\right. \\
\frac{d(\ln (N))}{d x}=-\sigma_{d} \frac{n_{\text {bons }}}{V}-\frac{1}{N} \cdot \frac{d N}{d x}=-\sigma_{d} \frac{n_{\text {ions }}}{V}- \\
d N=-\sigma_{d} N \cdot \frac{n_{\text {ions }}}{V} \cdot d x
\end{gathered}
$$

If we assume that the interaction region is rectangular with a cross sectional area $\mathrm{A}$, then $\mathrm{V}=\mathrm{A} . \mathrm{dx}$, and so from eq. 22 we can calculate the rate of photon absorption which is

$$
\frac{d N}{d t}=-\sigma_{d} \frac{N}{A d t} \cdot n_{\text {ions }} \text { or } \frac{d N}{d t}=-\sigma_{d} F \cdot n_{\text {lons }}
$$

where $\mathbf{F}$ is the photon flux and is defined as the number of photons crossing per unit time per unit area perpendicular to the photon beam direction. In addition, the rate of change in the photon absorption, must be equal (in absolute magnitude), to the rate of neutral formation $\mathrm{N}_{\text {neutrals, }}$ (i.e. $d N / d t=-N_{\text {neutrals }}$ ). Hence we can rewrite eq. 21 to obtain an alternative form of Beer's law which is ${ }^{7}$ : 


$$
N_{\text {newtrals }}=0_{d} F \cdot n_{\text {ions }} \text { or } \frac{N_{\text {neusrals }}}{n_{\text {bons }}}=\sigma_{d} F \text {. }
$$

The interaction time between a photon beam and an ion beam which cross at $90^{\circ}$, is often equivalent the transit time of the ion beam $t=l / N_{\text {ions }}, l$ being the width of the photon beam and $V_{\text {ions }}$ the speed of the ions. However, when pulsed lasers are involved, if this time $t$ is longer than the pulse length of the laser $\left(\Delta t_{\text {laser }}\right)$, then the interaction time is $t=\Delta t_{\text {laser }}$. In this case the total number of neutrals produced is

$$
n_{\text {tor }}=N_{\text {neurrals }} \cdot \Delta t_{\text {laser }} \text { or } n_{\text {too }}=\sigma_{d} F \cdot n_{\text {ions }} \cdot \Delta t_{\text {laser }}
$$

and if one were to completely neutralize the anion bunch ( $n_{-}$tot $=n_{-}$ions) this equation yields the

$$
F=\frac{1}{\sigma_{d} \Delta t_{\text {laser }}}
$$

Since the total number of photons per unit area $\left(\mathrm{N}_{\text {photons }}\right)$ is given by $N_{\text {photons }}=F \cdot \Delta t_{\text {laser }}$, eq 26 becomes

$$
N_{\text {photons }}=\frac{1}{a_{d}} \text {. }
$$

Assuming that $\sigma_{\mathrm{d}}$ is on the order of $10^{-18} \mathrm{~cm}^{2}$, then the total number of photons necessary for $100 \%$ photodetachment is $10^{18}$ photons $/ \mathrm{cm}^{2}$ per pulse. For a $3 \mathrm{eV}$ photon $(413 \mathrm{~nm})$ this corresponds to a per pulse photon energy of $\mathrm{I}_{\text {photon }}=0.5 \mathrm{~J} / \mathrm{cm}^{2}$ per pulse, which for a sqaure $2 \mathrm{~mm}$ beam corresponds to $\mathrm{I}_{\text {photon }}=5 \mathrm{~mJ} /$ pulse. These photon intensities can be acheived using pulsed dye lasers. In the situation where $\sigma_{d} \leq 10^{-19} \mathrm{~cm}^{2}$, the photon intensities required are $I_{\text {photon }} \geq 50 \mathrm{~mJ} /$ pulse, which can also be acheived, although not 
readily sustained (dye bleaching).

In light of the above it becomes obvious that space charge effects can be minimized if :

i. The width of the mass selected ion bunch $\Delta t_{i o n s}$ should be not exceed the width of the laser pulse :

$$
\Delta t_{\text {bons }}=\Delta t_{\text {laser }}
$$

ii. The ion density must be low enough so that the energy gains described by eq. 20 are less than $3 \mathrm{~cm}^{.1}$ (density ions $\leq 3 \times 10^{4}$ ions $\left./ \mathrm{cm}^{3}\right)$.

Finally we conclude that the ability to collect all the threshold electrons is the major factor in the success of ZEKE spectruscopy, because for systems with very small photodetachment cross sections. increasing the ion current will not increase the electron signal.

\section{Wigner's Law}

A very old quarrel between engineers and scientists is whether science causes technological advances or do technological advances result in scientific triumphs. In the previous paragraph we see an example where the perfection of an experiment is limited by the unavalability of certain high power lasers. Since this is a technological "bottleneck" there is always the possibility that it might be overcome with the help of science. However, when the bottleneck is a Physical Law, then we must either live with it, take advantage of it or work around it. In particular we are concerned with the threshold laws for photodetachment, that is the energy dependence of the photodetachment 
cross section when the photon energy is just above the minimum energy necessary to remove an electron.

Threshold laws in general are of great interest in several physical processes and have been addressed and formulated by Wigner ${ }^{12}$ in his landmark (bitchin) paper. For the photodetachment of an atomic negative ion, he found that

$$
\sigma \propto\left(E-E_{t h}\right)^{l+1 / 2}
$$

where $E_{\mathrm{th}}$ is the threshold energy, and $l$ is the angular momentum of the ejected photoelectron. This law was derived for atoms and it was assumed that long range forces govern the shape of the cross section near threshold. Wigner's approach was such that he ignored the details of the reaction zone, this being the region where the photoelectron is very close to the neutral product, and was concerned only with the final electronneutral configuration where the electron has sufficient energy to reach infinity; he then calculated the probability that subsequent evolution of the system will lead to ejection of an electron.

In deriving eq. 25 , Wigner assumed that the only long range force acting on the electron is the centrifugal repulsion $V=l(l+1) / r^{2}$. Following a review by $R a u,{ }^{13}$ we can gain sorne insight about eq. 25 from Fig. 4 where we have drawn the attractive short range potential (approximated by a square well) and the repulsive long range centrifugal potential. The effective potential $\left(\mathrm{V}_{\mathrm{eff}}\right)$ resulting from adding the two potential curves results in a barrier at energies just above threshold, which the electron has to tunnel through in order to escape. Fig. 4 illustrates that large values of angular momentum $l$ result in large barriers for which the probability of escape is reduced. By invoking 
symmetry arguments, the seiection rules for the photodetachment of atoms are $l=l_{i} \pm 1$, where $l_{i}$ is the orbital angular momenturn of the state from which the electron was ejected. For example if $l_{i}=0$ (s-orbital) then $l=1$ (p-wave detachment), while if $l_{i}=1$ (p-orbital), $l$ can take the values of 0 (s-wave process) or 2 (d-wave process).

In Fig. 5, the photodetachment cross section $\sigma_{d}$ vs the photoelectron kinetic energy $E_{c}$ is plotted, for $l=0$ and 1 ; it is obvious that near threshold $\left(E_{e} \leq 5 \mathrm{~cm}^{-1}\right)$, only the s-wave processes have a non-vanishing cross section. Since by virtue of our detection scheme we collect only ZEKE electrons, threshold photodetachment spectroscopy will only be successful if the species under investigation can undergo s-wave photodetachment. It is thus apparant that being able to predict the nature of the photodetachment process for an arbitrary system is important. For the case of atoms this is trivial and was described in the previous paragraph, we will address the molecular situations in the remainder of this chapter.

Geltman ${ }^{14}$ has shown that within the Born-Oppenheimer (B.O.) approximation, the photodetachment cross section near threshold for diatomics is given by

$$
\begin{gathered}
\sigma_{d} \propto k^{l+1} \\
\text { where } k=\frac{\sqrt{2 \cdot m_{e} \cdot E}}{\hbar}
\end{gathered}
$$

and so Wigner's law remains valid. The B.O. approximation postulates the separability between fast electronic motion and that of the slow moving nuclei. However, a ZEKE electron with $E_{e}=5 \mathrm{~cm}^{-1}$ will travel $1 \AA$ in $7 \times 10^{-15} \mathrm{sec}$, while the typical period for a nuclear vibration is $10^{-14} \mathrm{sec}$; it is obvious that the prerequisites of the B.O. approximation 
are not completely fulfilled near threshold. Nontheless, the validity of Wigner's law for diatomics is supported by our experimental results and will be presented in the following chapters. As far as selection rules, Geltman found that for homonuclear diatomics $\left(D_{m b}\right)$, photodetachment from a $u$ type orbital can give rise to an s-wave process, while for heteronuclear diatomics $\left(\mathrm{C}_{\mathrm{wv}}\right)$ the same is true when the elestron originates in a $\sigma^{+}$or $\pi$ orbital. Geltman treated the photodetachment of molecular negative ions in a similar way to the motion of an electron in a cylindrically electric field; hence we expect that similar selection rules should apply to linear polyatomics. However extending his analysis to less symmetric systems is very difficult and it is not surprising that this task was left as an exercise for the interested reader.

The behavior of the photodetachment cross section for polyatomic negative ions near threshold has been studied by Reed et al ${ }^{15}$ using an alterative approach to that of Geltman's. The authors showed that using symmetry arguments and making some reasonable assumptions, Wigner's Law can be apllied even to polyatomics. The most important assumptions made by Reed et al. were :

a. The validity of the Born-Oppenheimer approximation and absence of long range forces. b. The electron is ejected from the HOMO and can be treated using a one-electron approximation.

c. The photodetachment process does not disturb the symmetry of the wavefunctions which are not involved in the photodetachment.

d. The wavefunctions describing both the HOMO and the ejected photoelectron belong to an irreducible representation of the symmetry group of the molecule. 
e. The HOMO can be adequately described using a linear combination of atomic orbitals. Given the above assumptions, the selection rules for photodetachment of polyatomics are very similar to those of atoms: if the HOMO transforms like $x, y$ or $z$ (p-type orbital), then an s-wave or d-wave process can occur. Since the assumptions a-e are only approximately true, Reed concluded, as did Geltman, that Wigner's law is only approximately valid, and that a better description of the cross section is given by

$$
\sigma_{d} \propto k^{l+1} \cdot\left(1+O\left(k^{2}\right)+\ldots\right)
$$

The relative magnitudes of these higher order $k$ terms are generally not predicted by the Reed model, although we can expect their contributions to the cross section increase as assumptions a-e are increasingly violated.

The main problems with symmetry arguments is that they only allow or forbid certain physical process, but say nothing about its magnitude. Hence it is very difficult, and maybe even impossible to accurately predict the behavior of the photodetachment cross section near threshold for polyatomics with low symmetry, and one might only be able to correctly determine this, through detailed experimentation. The following chapters will be concerned with the results of photodetachment experiments we have performed on systems that do obey Wigner's law, and undergo s-wave photodetachment. 


\section{Figure Captions}

1. Velocity vector diagram for a photoelectron indicating the relationship between its velocity in the center of mass $\underline{v}_{e}$ and $\underline{v}_{e}(\mathrm{LAB})$ and laboratory frame.

2. Spatial discrimination between the ZEKE and energetic photoelectrons electrons.

3. Spatial focusing between the forward scattered and the ZEKE electrons.

4. Effective potential for Wigner's Law.

5. Photodetachment cross section vs photon near threshold for an s-wave and p-wave process. 


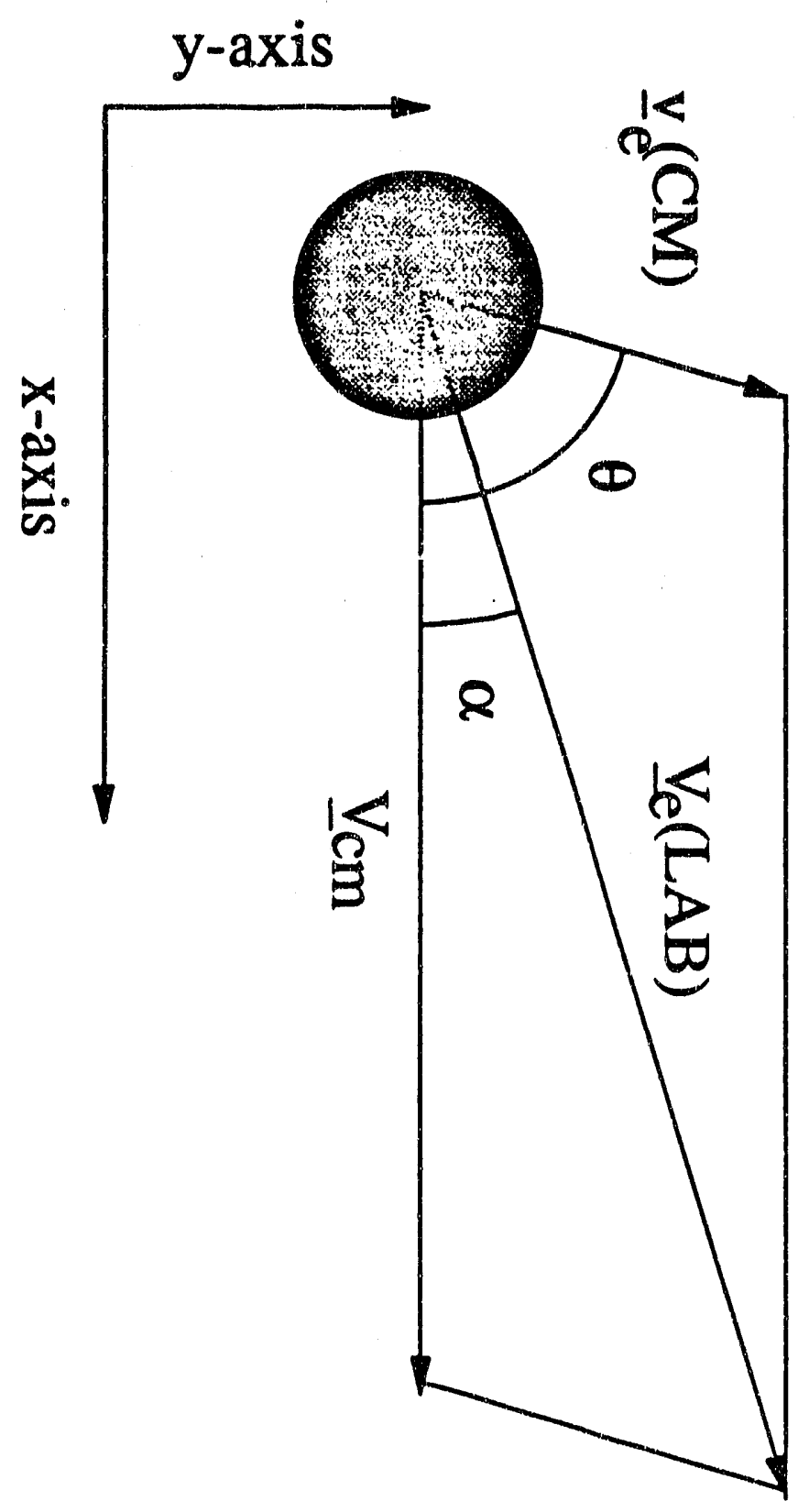

Figure 1 
High energy electrons

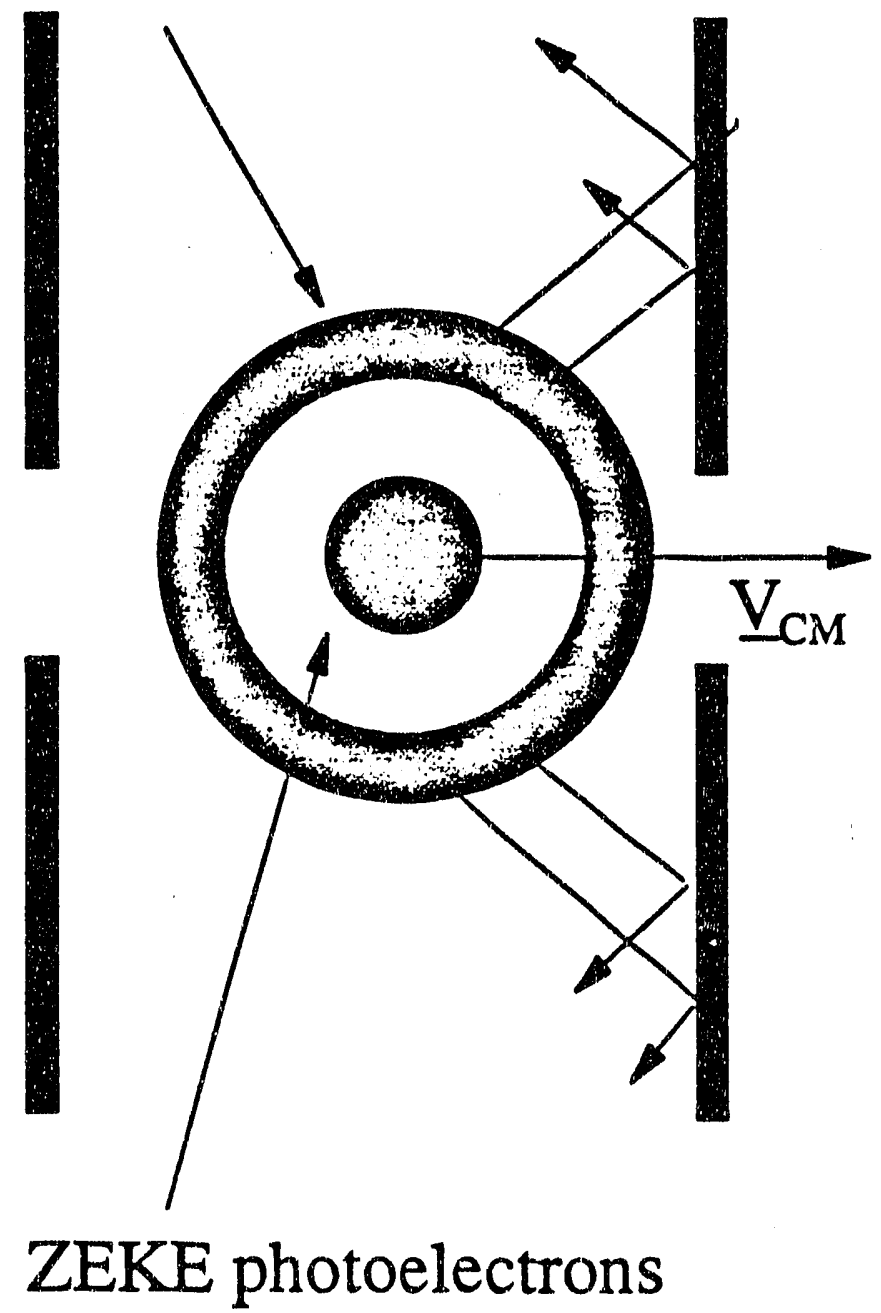

Figure 2 

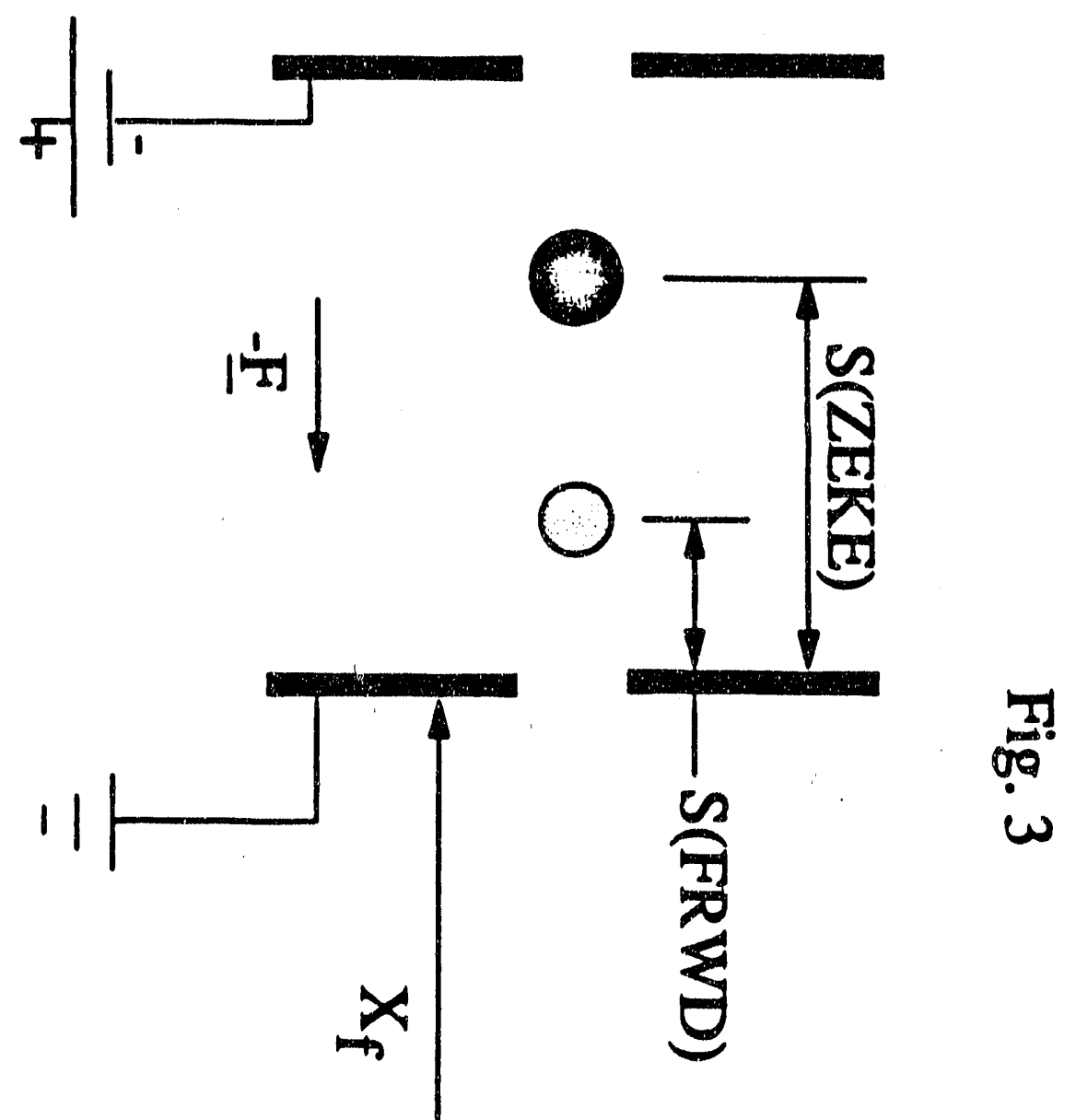


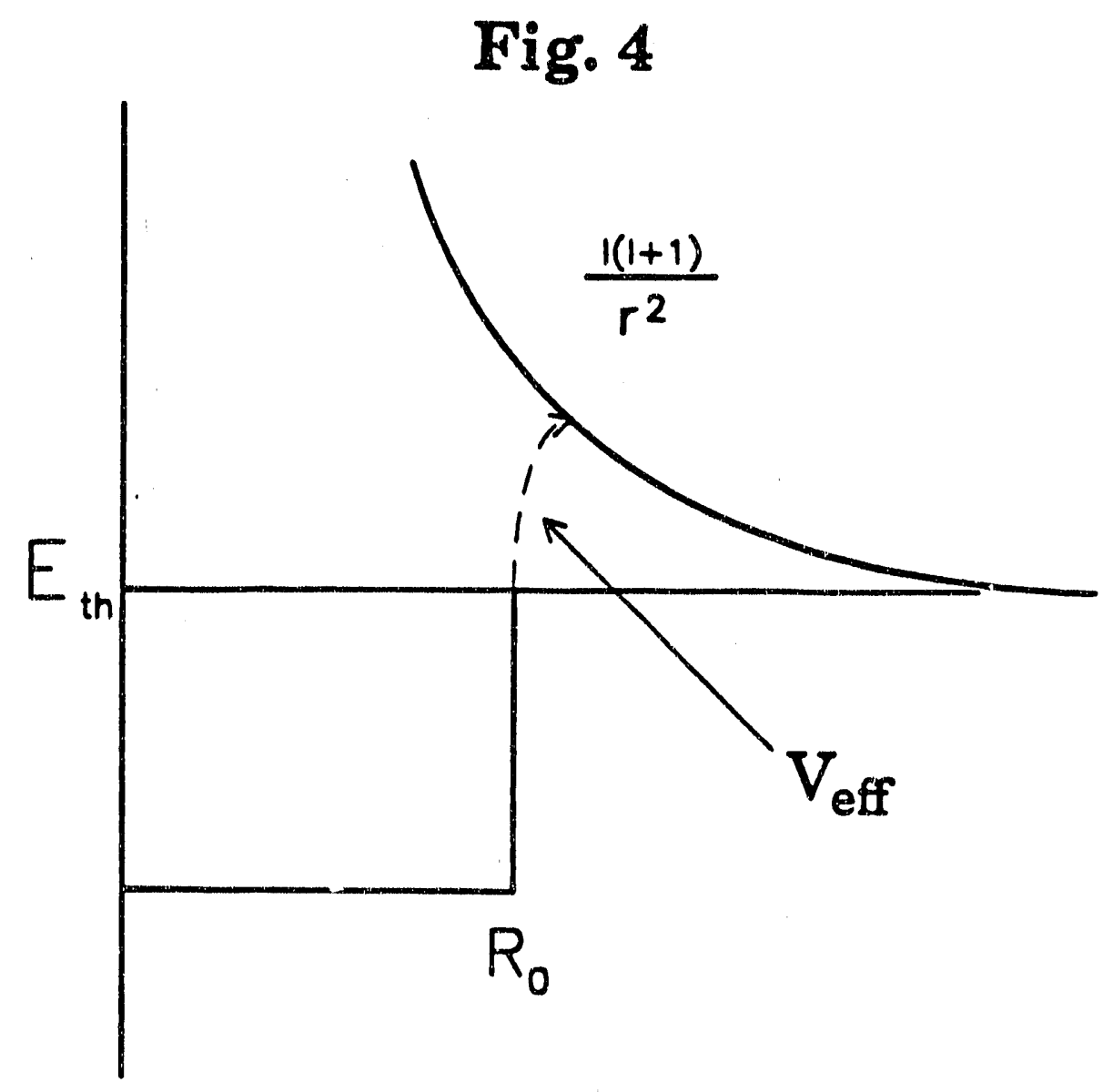




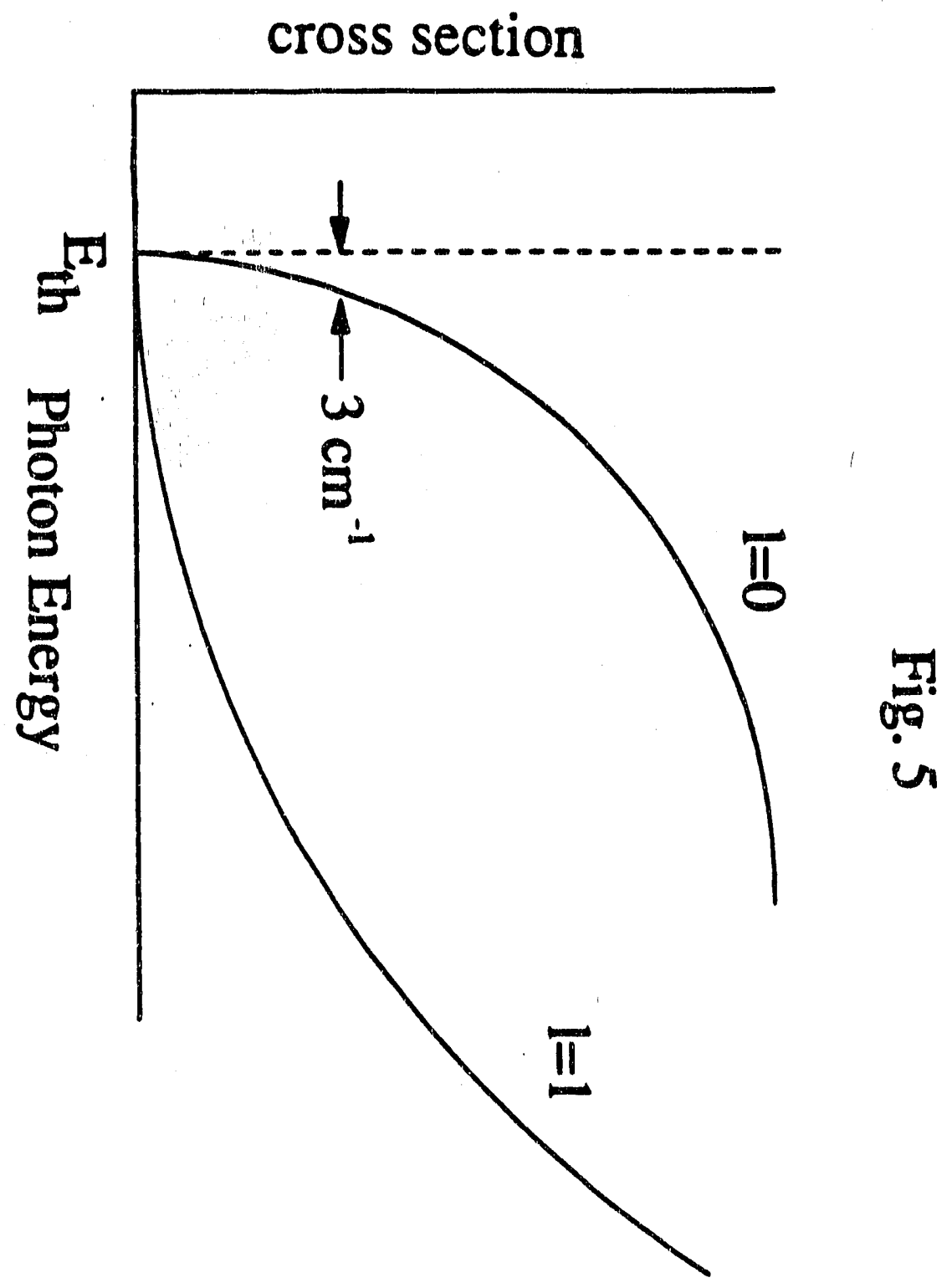




\section{References}

1. A. Einstein, Ann. d. Phys. (17), 132 (1905).

2. H.B. Ellis and G.B. Ellison Jr., J. Chem. Phys. 78, 6541 (1983); D.G. Leopoldm K.K. Murray, and W.C. Lineberger, J. Chem. Phys. 81, 1048 (1984); R.D. Mead, A.E. Stevens, and W.C. Lineberger, Gas phase ion chemistry, Vol. 3, ed. M.T. Bowers (Academic Press, New York), p. 213 (1984).

3. J.V. Coe, J.T. Snodgrass, C.D. Friedhoff, K.M. Mchugh and K.H. Bowen, Chem. Phys. Lett. 124, 274 (1986); D.G. Leopold, J. Ho, and W.C. Lineberger, J. Chem. Phys. 86, 1715 (1987); O. Chesnovsky, S.H. Yang, C.L. Pettiette, M.J. Craycraft, Y. Liu, and R.E. Smalley, Chem. Phys. Lett. 138, 119 (1987); M.J. DeLuca, B. Niu, and M.A. Johnson, J. Chem. Phys. 88, 5857 (1988).

4. R.B. Metz, T.N. Kitsopoulos, A. Weaver, and D.M. Neumark, J. Chem. Phys. 88, 1463 (1988); A. Weaver, R.B. Metz, S.E. Bradforth, and D.M. Neumark; R.B. Metz, A. Weaver, S.E. Bradforth, T.N. Kitsopoulos, and D.M. Neumark, J. Chem. Phys. 94, 1377 (1990).

5. A. Weaver, R.B. Metz, S.E. Bradforth, and D.M. Neumark, J. Chem. Phys. 90, 2070 (1988).

6. D.G. Leopold, K.K. Murray, A.E. Stevens Miller, and W.C. Lineberger, J. Chem. Phys. 83, 4849 (1985).

7. H. Hotop and W.C. Lineberger, J. Phys. Chem. Ref. Data 14, 731 (1985).

8. A $2 \mathrm{meV}$ resolution was reported by S.W. Allendorf, D.J. Leahy, Dennis.C. Jacobs, and R.N. Zare, J. Chem. Phys. 91, 2216 (1989). 
9. K. Müller-Dethlefs, M. Sander, and E.W. Schlag, Z. Naturforsch. 39, 1089 (1984); K. Müller-Dethlefs, M. Sander, aysd z.W. Schlag, Chem. Phys. Lett. 12, 291 (1984); M. Sander, L.A. Chewter,K. Müller-Dethlefs, and E.W. Schlag, Phys. Rev. A 36, 4543 (1987).

10. T.N. Kitsopoulos, I.M. Waller, J.G. Loeser, and D.M. Neumark, Chem. Phys. Lett. 159, 300 (1989).

11. W.C. Wiley and I.H. McLaren, Rev. Sci. Instr. 26, 1150 (1955).

12. E.P. Wigner, Phys. Rev. 73, 1002 (1948).

13. A.R.P. Rau, Comments At. Mol. Phys. (Gordon and Breach Science Publishers Inc. 1984), Vol. 14, pp. 285-306.

14. S. Geltman, Phys. Rev. 112, 176 (1958); L.M. Branscomb, D.S. Burch, S.J. Smith, and S. Geltman, Phys. Rev. 111, 504 (1958).

15. K.J. Reed, A.H. Zimmerman, H.C. Anderson, and J.I. Brauman, J. Chem. Phys. 64, 1368 (1976). 


\section{Chapter II}

\section{A Negative Ion Threshold Photodetachment Spectrometer}

\section{Introduction}

This chapter will be concerned with the experimental details of the negative ion threshold photodetachment spectrometer used in our work. ${ }^{1}$ At the end of the chapter, we will demonstrate the performance of the instrument by presenting the threshold photodetachment spectra of $\mathrm{I}^{-}$and $\mathrm{SH}^{-}$. In the latter spectrum, individual ion $\rightarrow$ neutral rotational transitions were resolved as peaks in the spectrum; in effect we have obtained the rotational resolved photodetachment spectrum of a negative ion

A schematic of the apparatus is shown in fig. 1, and consists of three regions: the source, the mass spectrometer, and the detector. In the source region the negative ions are produced by electron bombardment of a neutral expansion or by laser vaporization. The beam of negative ions passes through a skimmer in order to select out the central, coldest part of the expansion, and then enters the mass spectrometer region.

As we mentioned in chapter I, one of the big advantages of negative ion techniques, is that they allow for mass selection prior to spectroscopic interrogation. In this apparatus, we use a collinear time of flight spectrometer to mass select our negative ion beam. The term collinear comes from the fact that the ion beam is accelerated in a direction parallel to the direction of the initial neutral/ion expansion.

Following mass selection, ions enter the detector region where their threshold photodetachment spectra are obtained. The design of the detector region is based on the principles of threshold photodetachment spectroscopy discussed in chapter I. In brief, the 
negative ions are phototetached using a tunable dye laser and ZEKE (threshold) electrons are then collected as a function of photon energy. A complete description of the source, the mass spectrometer and the detector region will be presented in the following sections.

\section{Source region}

The source region is a rectangular chamber ( $\left.24^{\prime \prime} \times 24 " \times 16^{\prime \prime}\right)$ made of aluminum and is pumped by a $10^{\prime \prime}$ Edwards diffstak which maintains a background chamber pressure of $4 \times 10^{-6}$ Torr. Located in the source are: a pulsed molecular beam, an electron gun, an optional laser vaporization source which mounts on the pulsed beam, a Faraday cup, and electrostatic shielding. All the elements in the source region can be "floated" at some negative potential with respect to the earths ground; this allows us to generate anions at some desired potential and accelerate them up to ground.

\section{a. Molecular Beam}

The pulsed molecular beam used in our experiments is a single solenoid valve made by General Valve Corp. (series 9). The operation principles of the valve aie based the properties of solenoids and magnetic circuits. ${ }^{2}$ For a solenoid whose length is much larger than its radius, the magnetic induction B within the solenoid is given (approximately) by the equation:

$$
B=\frac{\mu_{0} \cdot N . I}{L}
$$

where $N$ is the number of coil turns, $I$ is the current, $L$ is the solenoid length and $\mu_{0}$ is the magnetic permeability of vacuum. In fig. 2 a magnetic rod with a constant magnetic permeability $\mu$, and cross sectional area $A$, is positioned such that only half of its length 
remains in a solenoid. In this configuration, the rod will feel force tending to pull it back into the solenoid. We can calculate this by determining the magnetic energy of the rod. This is given by

$$
U_{s}=\frac{1}{2} \int_{V} \mu \cdot H^{2} d V
$$

where $\mathrm{H}$ is the magnetic field and $\mathrm{V}$ is the volume of the rod within the field. Using this equation, the relation $B=\mu . H$ and eq.1, we find that the energy change for a small displacement of the rod $\Delta x$ is given by

$$
\Delta U=\frac{1}{2} \int_{A \Delta x}\left(\mu-\mu_{0}\right) \cdot H^{2} \cdot d V
$$

which becomes

$$
\Delta U=\frac{1}{2}\left(\mu-\mu_{0}\right) \cdot \frac{N^{2} \cdot I^{2}}{L^{2}} A \cdot \Delta x
$$

. The force on the rod is then given $b y^{2}$

$$
\begin{gathered}
F_{s}=\frac{\Delta U}{\Delta x}- \\
F_{s}=\frac{1}{2}\left(\mu-\mu_{0}\right) \cdot \frac{N^{2} \cdot I^{2} \cdot A}{L^{2}}
\end{gathered}
$$

and its direction is towards the center of the solenoid, shown in fig. 2 .

A schematic of the yalve is shown in fig. 3 and consists of a coil, a magnetic armature, two springs and a poppet. A driver circuit (DRVR) for the valve introduced to us by Prof. Mark Smith (Univ. of Arizona), is shown in fig. 4. The valve is normally shut, and in this position spring-A pushes the poppet against the face plate orifice, while 
spring-B prevents the armature from overriding the poppet. The valve opens when a current passes through the valves coil creating a magnetic field which pulls the armature/poppet away from the face plate, thus allowing gas to flow through the valve orifice. How long the valve remains open is determined by the length of the TTL openpulse generated by a 74123 monostable, and can be adjusted externally using a 10-turn trim pot; this open-pulse turns $O N$ transistor $Q_{2}$, which in turn drives transistor $Q_{1}$ into conduction, thus establishing a current through the valve solenoid.

Their are two stages during the operation of the driver circuit: the steady state stage and the "transient" stage. During the steady state stage the current $I_{d c}$ is constant, and coincides with the current required to maintain the valve open. The manufacturers recommended current $\left(I_{d c}\right)$ for the series $\#$ General Valve is 0.15 Amps, and hence we chose resistor $R_{1}$ from Ohm's Law

$$
I_{d c} \approx \frac{300 V}{R_{1}}=0.15 \text { Amps }-R_{1}=2 \mathrm{~K} \Omega .
$$

The "transient" stage is concerned with the period during which the capacitor $C_{1}$ is discharging, which occurs durning the initial turning $O N$ of $Q_{1}$. The risetime of this stage is identical to the time required to establish a current equal to $I_{d c}$. Typical (as well as desirable) transient risetimes for our driver circuit are on the order of $100 \mu \mathrm{sec}$, which means that the total amount of charge delivered by the capacitor $C_{1}$ will be

$$
Q_{\text {roc }} 2 I_{d c^{\prime}}(100 \mu \mathrm{sec})=15 \times 10^{6} \mathrm{C} .
$$

A lower limit on the capacitance $C_{1}$ is then given by 


$$
C_{1} \geq \frac{Q_{\text {tot }}}{300 V}=0.05 \mu F
$$

An ideal pulsed molecular beam is that which generates intense pulses of gas in a very short time. For the General Valve the limiting factors are how fast can the armature/poppet move away from the face plate, and how fast can the spring-A return the system to it's shut position. If we assume that spring-A is always operating in its $\underline{\text { linear }}$ regime, then for any given armature displacement (typically .001" to $.005 ")$, both the opening and closing times will be depend heavily on the natural constants of the valve (mass of armature/poppet, friction, spring force constant etc.). In addition, since the force electromagnetic force on the armature is directly proportional to the current (eq. 5), the valve opening time will also dependent critically on how fast the steady state current $I_{d c}$ established. Since our driver is essentially an $R-L-C$ circuit, the transient current $I(t)$ will be given $b^{2}$

$$
\begin{gathered}
I(t)=\sqrt{\frac{V}{\omega_{n} \cdot L_{c}}} \cdot \exp \left(-\frac{R \cdot t}{2 L_{c}}\right) \cdot \sin \left(\omega_{n} \cdot t\right) \\
\text { where } \quad \omega_{n}=\sqrt{\frac{1}{L_{c} \cdot C_{1}}-\frac{R^{2}}{4 L_{c}^{2}}}
\end{gathered}
$$

where $L_{c}$ is the inductance of the valve solenoid and $\omega_{\mathrm{n}}$ is called the natural frequency of the circuit.

From the appearance of eq. 9 , one observes that large values of $C_{1}$ will produce large currents and perhaps more efficient (faster) valve opening, but they also cause extensive heating of the valve body. The reason for this is that once the minimum 
required current $(0.15 \mathrm{~A})$ for complete opening of the valve has been established, any excess current will resistively heat up the valve. The heat $(Q)$ absorbed by the valve is

$$
Q=\int_{0}^{T_{\operatorname{son}}} I(t)^{2} \cdot R d t
$$

where $I(t)$ and $R$ are the current and resistance across the valve coil, and $T_{\text {open }}$ the openpulse length. Small $C_{1}$ capacitors discharge quickly, so most of the heat will be dissipated during the steady state stage. We can approximate eq. 10 by

$$
Q \propto I_{d c}^{2} \cdot R \cdot T_{\text {open }}
$$

and so for a repetition rate of $f_{R e p .}$. the total power dissipation is

$$
P_{\text {sot }}=Q \cdot f_{\text {Rep }} \alpha I_{d c}^{2}, R \cdot T_{\text {open }} \cdot f_{R e p} \text {. }
$$

For the minimum required steady state current $I_{d c}=0.15 \mathrm{~A}$, a $70 \Omega$ valve coil, a repetition rate of $100 \mathrm{~Hz}$, and an open pulse duration of $250 \mu \mathrm{sec}$, we find

$$
P_{\text {tot }}=0.04 \text { Watts }
$$

which results in negligible heating. Large capacitors $\left(C_{1} \geq 0.5 \mu F\right)$ never discharge completely, and so average currents of $I_{d c} \geq 1 \mathrm{Amp}$ can be delivered, which for the same conditions described above results in a heating rate of

$$
\mathrm{P}_{\mathrm{tot}}=1.75 \text { Watts }
$$

which results in excessive heating of the valve. Experimentally we have established that a value of $C_{1}=0.1 \mu F$, allows adequate gas throughput, and negligible heating.

The force exerted on the armature is described by eq. 5 . In deriving this equation we assumed a constant current situation which is not entirely true during the transient 
period; by simply substituting $I$ by $I(t)$ in eq. 5 , one obtains:

$$
F_{s}(t)=\frac{1}{2}\left(\mu-\mu_{0}\right) \cdot \frac{N^{2} \cdot A}{L^{2}} \cdot I^{2}(t)
$$

which is the appropriate expression for the "transient" stage. This time dependent force appears to be independent of the extent of the armature within the coil. However, changing this extent will change the inductance $\left(L_{c}\right)$ of the valve solenoid, which in turn will affect $I(t)$. In general, because the armature is a ferromagnetic material, the larger the armature extent the larger the inductance. In addition, since the magnetic permeability of the armature is at least $10^{3}$ times larger than that of air, even the smallest variations of the armature extent will affect the coil inductance drastically. Small $L_{c}$ values will result is slow transient current rise times $\left(\mathrm{R} / 2 L_{c}\right.$, eq. 10), and hence an inefficient valve opening. Experimentally when face plate of the valve is loose, which implies that the armature extent is very small, the valve does not open (minimal throughput).

The optimum initial position (i.e. fastest rise time and maximum gas throughput) of the armature can be experimentally determined as follows:

a. Set the open-pulse duration to the desired length.(Recommended value is $250 \mu \mathrm{sec}$ ). b. Set the backing pressure to the desired operating value.

c. Maximize the gas throughput by tightening the face plate onto the threaded body of the valve.

Some additional valve operating tips are:

1. Poppets are made by either KEL-F or TEFLON. Although Teflon poppets are soft and thus do not leak, their tips do deform with relative ease resulting in very short life 
times. KEL-F is a very hard material and hence has the tendency to leak if the face plate orifice is not completely smooth. Because of its durability however and consequently its long life time, it is the recommended poppet.

2. Because the poppets are tapered, they extend within the valve orifice substantially, and thus resulting in an "effective" nozzle diameter that is smaller than the diameter of the orifice. It is not uncommon for a 0.010 " orifice to have more gas throughput than a $0.020^{\prime \prime}$ orifice given the same operating conditions, mainly because the effective nozzle for the former can actually be greater than the later. Beyond 0.020 " the throughput does appear to increase as the nozzle size gets bigger.

3. Fluctuations in the gas throughput are often caused by friction between the armature and the valve body. Most armatures come with a protective teflon coating which serves as a lubricant and prevents galling. We find that applying a small layer of KRYTOX grease to the armature also facilitates the armature motion, thus contributing to the shot-to-shot stability of the valve.

4. The magnetic properties of the armature do deteriorate with time and can cause a substantial decrease in the throughput of the valve.

5. Fixing the position of the faceplate by either a set screw or, preferably, using shims, increases the longevity and stability of the valve. This procedure is mandatory when accessories are supported by the valve (i.e. laser vaporization sources, expansion/mixing channels etc.).

\section{b. Electron Gun/Negative Ion Production}

Negative ions in our laboratory are generated with one of two methods: Electron 
bombardment of a neutral expansion $^{3}$ and laser vaporization. In this section we will concentrate on the former. Electrons used in the electron bombardment are generated by a modified TEKTRONIX electron gun (e-gun) shown in fig. 6. The e-gun is capable of producing a collimated continuous beam of electrons with energies up to $1 \mathrm{keV}$ and typical current yields between 100 and $500 \mu \mathrm{A}$. The operation principles, of the e-gun are: 1. Electrons are generated by resistively heating a Thoria coated Iridium filament. Typical filament operating currents are 4-6 Amps at 7-10 V, and the current is measured using a Faraday cup made of copper.

2. Generated electrons are then accelerated by the capacitance type field, formed between the anode and the first element of the e-gun einzel lens. This is accomplished by floating both the filament and the anode at a desired potential (typically $-800 \mathrm{eV}$ to $1000 \mathrm{eV}$ ) with respect to the first einzel lens element which is at $V_{\text {four. }}$ Because the electrons generated by the filament are ejected at random directions, a small bias voltage on the anode with respect to the filament potential, typically $\pm 0-100 \mathrm{~V}$, is used to guide as many electrons as possible into the "acceleration region", thus increasing the current yield of the e-gun. When the filament tip sits low (retracted) with respect to the anode face, fig. 7(a), a positive bias voltage is used in order to attract the electrons towards the anode orifice. Conversely a negative bias voltage is used when the filament tip sits high (protrudes) with respect to the anode face, fig. 7(b), because the electrons need to be repelled away from the anode orifice. For the low filament situation, the importance of the anode biasing is demonstrated in fig. $7 c$, where one observes that failing to bias the anode results in poor electron beam quality. These electron trajectory 
imulations were obtained using the program SIMION ${ }^{4}$; since this program runs single particle trajectories, it does not take into acount "space charge" effects, due to the electrostatic repulsions between the charged particles of the electron (or ion) beam.

3. The electron beam is collimated using an series of apertures which are part of elements of the e-gun einzel lens. The beam can the be stirred using two sets of deflectors and spatially focused using the decelerating einzel lens just mentioned.

The electron gun is mounted in a direction perpendicular tc the molecular beam direction as shown in fig. 5. The electron beam crosses the neutral expansion produced from the pulsed valve at a distance very close to the valve nozzle. This is desired so that the negative ions are generated in the continuum flow region of the expansion and hence will themselves undergo supersonic cooling. The dependence of anion internal temperature on the position of the electron-neutral beam crossing point (with respect to the nozzle) has not been established experimentally, and varies from system to system. Typical crossing distances lie between $1 \mathrm{~mm}$ and $15 \mathrm{~mm}$.

The crossing of the electron beam with the neutral expansion described above can produce electrons via several mechanisms:

a) Dissociative electron attachment

$$
e+A B \rightarrow A^{-}+B
$$

b) Electron attachment

$$
\begin{gathered}
e+A B \rightarrow(A B)^{\circ *} \\
(A B-)^{* *}+M \rightarrow A B^{-}+M
\end{gathered}
$$

The electrons involved in both mechanisms are slow electrons generated from the 
collision of the electron beam with the molecular beam. Excellent reviews on production of negative ions have written by H.S.W. Massey, ${ }^{5}$ B.M. Smirnov ${ }^{6}$ and L.G. Christophorou. $^{7}$ Details concerning the production of specific negative ions will be described in the appropriate chapters that follow.

\section{c. Floating Scheme}

As we mentioned earlier, in order to generate a fast ion beam, we need to produce the anions at some negative voltage and then accelerate them to ground. We accomplish this by floating the source region, that is, making sure that the electrical potentials for every component of the source chamber are at some negative voltage with respect to the earths ground. Hence one must float both the chassis (bodies) of the source components hardware, as well as their corresponding electrical and electronic circuitry. Since the walls of the source chamber are themselves grounded, components are supported by insulating materials such as DELRIN or VESPEL, using clever mechanical designs. DELRIN has poor outgassing properties ${ }^{8}$ but is inexpensive and is the preferred material when one is dealing with low vacuum $\left(10^{-4}\right.$ to $10^{-7}$ Torr); VESPEL which has excellent outgassing properties should be exclusively used for UHV applications (in spite of its cost). To maintain a field free environment (at the float equipotential) within the source region, we use a "double wall" configuration like the one shown in fig. 5; this "inside" wall is comprized of solid metal sheets or grids which are floating at the desired source potential $\left(V_{\text {noul }}\right)$, and thus shield the source components from the grounded chamber walls.

Electrical isolation of the filament and electron gun power supplies is achieved through using isolation transformers. A generic transformer consists of two coils, 
electrically isolateci, but situated such that an ac-current in one will electromagnetically induce a potential $\left(\mathrm{EMF}_{2}\right)$ on the other (see fig. 8). One coil is referred to as the primary and the other as the secondary. Consider next a generic 1:1 transformer shown in fig. 8a, where we have connected the primary side to an $110 \mathrm{~V}$ ac power supply. The primary is also grounded ( $\mathrm{dc}$ bias) through a pair of equal large resistors $(\geq 1 \mathrm{M} \Omega$, using a large resistor minimizes the dc current). Any device powered by this primary side will be operating a $110 \mathrm{~V}$ ac, and $0 \mathrm{~V}$ dc i.e. the device will be grounded. The induced EMF on the secondary side will also be $110 \mathrm{~V}$ ac, however its dc potential is floating, in other words has NO reference. If we connect the outputs of the secondary side to a dc power supply as shown in fig. $8 \mathrm{~b}$, then the any device connected to the secondary side will receive $110 \mathrm{~V}$ ac, however its dc bias will be determined by the float voltage $\left(\mathrm{V}_{\text {now }}\right)$. The maximum allowed value of $\mathrm{V}_{\text {float }}$ is determined by the transformer rating.

Fig. 9 illustrates the floating scheme of our source region. Two isolation transformers were used (highlighted in grey circles), since it was necessary to float the electron filament/anode with respect to the float voltage (section 1.b.). In the experiments that will be described in the following chapters float voltages of $-500 \mathrm{~V}$ and $-1000 \mathrm{~V}$ wwere used.

\section{d. Laser Vaporization Source}

An alternative method for generating negative ions, particularly cluster anions, is the laser vaporization method. ${ }^{9}$ In this situation, a laser beam if focused onto the surface of a rotating and translating sample rod. This causes intense local heating which vaporizes part of the surface creating a plasma consisting of neutral and charged particles 
(both positive and negative ions). ${ }^{10}$ The plasma is then entrained in a pulse of He from a molecular beam and expanded through a channel into a vacuum chamber.

A typical laser vaporization source (LAVAS) is illustrated in fig. 10, where one observes two main regions: the clustering region located inside the LAVAS, and cooling region located ouside the LAVAS. Following vaporization the atomic vapor undergoes many collisions with neighboring atoms to form clusters. The longer the clustering channel the more collisions will occur and hence the distribution of clusters will shift to higher masses. During the formation of a cluster there is a substantial amount of energy released which must be dissipated through a third body :

$$
\begin{aligned}
M+M_{n} & \rightarrow\left(M_{n+1}\right)^{*} \\
\left(M_{n+1}\right)^{*}+\mathrm{He} & \rightarrow M_{n+1}+\mathrm{He} .
\end{aligned}
$$

To achieve efficient cooling it is often necessary to use intense pulses of He, and consequently the backing pressures behind the valve nozzle as high as 5-10 atm are used. As we will discuss in later chapters, we find that the electronic and vibrationally coldest anion clusters are generated if the vaporization occurs when the density of He pulse over the sample rod is the greatest.

Although a great deal of cooling takes place within the clustering channel, the lowest "internal" temperature one can acheived would be room temeprature. Supersonic cooling $(0-100 \mathrm{~K})$, is achieved in the region immediately after the LAVAS nozzle, where the cluster cloud is expanded into a vacuum. The internal temperature of the clusters produced is critically dependent on the expansion quality. Although some clustering can also occur in this region, as we mentioned before the size distribution is almost 
exclusively determined by the length of the clustering channel." Using argon or neon as a carrier gas does seem to cool the clusters more efficiently, but He expansions seem to produce sufficiently cold anion clusters in a much greater abundance and are thus preferred.

For the laser vaporization we have used both the second harmonic of Nd:YAG $(532 \mathrm{~nm}, 20 \mathrm{~Hz})$ and the output of a XeCl excimer laser $(308 \mathrm{~nm}, 20-60 \mathrm{~Hz})$. The laser beam was focused using a $1 \mathrm{~m}$ or $50 \mathrm{~cm}$ focal length lens; we find the $1 \mathrm{~m}$ lens to be experimentally advantageous, since its beam "waist" near the focus point is considerably longer (in the direction of beam propagation), than for the $50 \mathrm{~cm}$ one. Thus the position of lens relative to the sample rod is not as critical, and so alignment of the vaporization beam is greatly facilitated. Because we are focusing the laser beam down to a $1 \mathrm{~mm}$ spot, unstable resonator optics must be used with the $\mathrm{XeCl}$ excimer laser, otherwise the output beam quality is not suitable ior tight focusing.

Laser intensities necessary for efficient vaporization are typically low, ranging from 3 to $25 \mathrm{~mJ} / \mathrm{pulse}$, and depend on the nature material that is being vaporized. We find that when excessive laser fluences are used, the vibrational and electronic temperatures of the cluster anions is considerably high. In addition, very hot clusters will undergo dissociation and so the cluster yield and shot to shot stability of the LAVAS are also reduced when the laser power is too high.

It became apparent that being able to control the laser fluence is very critical. In the case of the $\mathrm{XeCl}$ excimer, where the laser beam is randomly polarized, this was done by using a series of attenuators made from fine stainless still mesh. Although this is a 
very "coarse" adjustment it was successful. In the case of the Nd:YAG, since the output is plane polarized, we used a polarizer in series with a half wave plate to adjust the power output and this scheme is shown in fig. 11. The laser passes through the half wave plate which rotates its polarization; the laser beam subsequently passes through a polarizer which allows only the horizontal component of the laser beam through, and reflects the rest. $^{12}$ By rotating the half wave plate one can get a fine, continuous adjustment of the laser fluence and the intensity of the output beam is given by Malus's Law :

$$
I_{\text {out }}=I_{\text {in }} \cdot \cos ^{2}(\theta)
$$

which was first published in 1809 (!) by Etienne Malus, ${ }^{12}$ military engineer and captain in the army of Napoleon.

The ability to adjust the laser intensity in a very controlled fashion using the Nd:YAG laser has made it the preferred vaporization system in our laboratory. One of its limitations is the low repetition rate, limited to only $20 \mathrm{~Hz}$ as opposed to the $100 \mathrm{~Hz}$ offered by the excimer. However when using the LAVAS the gas throughput is often so intense that for our source chamber pumping speed, it is very difficult to run the pulsed valve at repetition rates much higher then $30 \mathrm{~Hz}$.

Although there has been a fair amount of discussion concerning the wavelength dependence of the LAVAs's ionic cluster yields, at this point there has been no conclusive experimental eviderce which seems to support any such dependence. The vaporization laser is purely a "heat source" for the rod surface, and since the radiation used is far above the infrared (where we would be exciting specific surface vibrational modes of the sample rod), we do not expect any severe dependence of the heating rate 
on the wavelength.

Because the pulse width of the vaporization laser in on the order of $5 \mathrm{nsec}$, the width of the cluster pulse produced is very narrow. If the spatial extent of the laser beam is approximately $1 \mathrm{~mm}$, and the average stream velocity acquired by the clusters due to the He carrier pulse is on the order of $10^{5} \mathrm{~cm} / \mathrm{sec}$, then time width of the cluster distribution due solely to the spatial extent of the laser is :

$$
\Delta t=\frac{S_{\text {laser }}}{\left\langle\nu_{\text {cluster }}\right\rangle}=1 \mu \mathrm{sec}
$$

$$
\text { when } S_{\text {laser }}=1 \mathrm{~mm} \text { and }\left\langle v_{c \text { clussar }}>=10^{5} \frac{\mathrm{cm}}{\mathrm{sec}}\right. \text {. }
$$

This is true when pressure of He carrier gas is low, and is observed experimentally when the laser vaporization occurs too soon i.e. during the early part of the buffer gas pulse. Consider now a typical nozzle to skimmer distance of $4 \mathrm{~cm}$; given a stream velocity of $10^{5} \mathrm{~cm} / \mathrm{sec}$, the clusters will arrive at the skimmer within $40 \mu \mathrm{sec}$. If we assume that there is a $2 \%$ fluctuation in the stream velocity (i.e. $\left\langle\mathrm{v}_{\text {clusters }}\right\rangle=1 \pm 0.02 \times 10^{5} \mathrm{~cm} / \mathrm{sec}$ ), then the variation in the arrival time will be $\Delta t= \pm 1 \mu \mathrm{sec}$, which is comparable to the width of the clusters. This "timing jitter" which results from the relatively narrow distribution of the cluster plasma, can result in instabilities in our mass selected ion cluster beam. For example, as we will explain in section 2 below, mass selection of the ion beam is achieved by reversing the direction of a field formed by two parallel plates (Bi:AP), when the ion cloud is located mid-way through the plates. Variations in the arrival times of the ion cloud at the BMP will consequently cause instabilities in the intensities of the mass 
selected ion packets.

One way to "spread out" the pulse is by using a "waiting room", introduced by Smalley and coworkers ${ }^{13}$ and is shown in fig 10. This is a modified clustering channel whose volume is very large following the vaporization region, and whose exit hole to the vacuum chamber is a relatively small orifice. The residence time of the cluster/He pulse within this "waiting" room depends on the dimensions of the channel. For cylindrical geometries, where the ratios of the "waiting room" diameter (0.120") to the exit orifice $\left(0.060^{\prime \prime}\right)$ is $2: 1$, we have observed residence times between 50 and $100 \mu \mathrm{sec}$. This residence time allows additional He/cluster mixing and will cause the cluster distribution to spread out. The new width will be on the order of the residence time (50 to $100 \mu \mathrm{sec}$ ), which thus eliminates the "timing" problem introduced by the fluctuations in the stream velocity of the beam. Furthermore we should mention that using just an extended clustering channel ( 1 to $2 \mathrm{~cm}$ long, and $2 \mathrm{~mm} \mathrm{I.D.)}$, also results in a spreading out of the cluster pulse, comparable to the that of the "waiting" room. Since the two geometries produce slightly different cluster distributions, the preferred clustering channel is that which most readily generates the cluster of interest. In general, the waiting room is used when large clusters are being investigated.

The "waiting" room also provides additional time for the clusters to cool down to room temperature, due to the increased number of collisions that can occur between the He buffer gas and the cluster plasma. However, we have observed that the internal temperature distribution of the cluster pulse is not uniform. Specifically, we find that clusters generated within the leading part of the expansion yield vibrationally colder 
spectra. This might have to do with the way the "waiting" room functions. Initially the pressure of the He pulse within the channel is high (about $1 \mathrm{~atm}$ ), and so the flow of gas through the channel is hydrodynamic (viscous), yielding a supersonic expansion (cold clusters). ${ }^{14,15}$ As the buffer gas pressure within the "waiting" room drops, the expansion becomes less supersonic and more effusive, ${ }^{14,15}$ hence yielding internally hotter clusters.

\section{Mass Spectrometer}

In this region of the spectrometer, the anion beam is mass selected using a collinear time-of-flight mass spectrometer. ${ }^{16}$ This region consists of two differentially pumped regions and is illustrated in fig. 12. The region immediately behind the skimmer contains an ion stack lens used to accelerate the ions, and an electric field formed by two parallel plates called beam modulation plates (BMP), which are components of the mass spectrometer. The $2 \mathrm{~mm}$ skimmer also allows differential pumping of this region from the source region. This 1 st differential region is pumped using an 6" Edwards Diffstak; background pressures of $8 \times 10^{-8}$ Torr are achieved, while typical operating pressures vary between $1 \times 10^{-6}$ and $1 \times 10^{-5}$ Torr.

The initial region of the second differential constitutes an ion einzel lens used to correct for any spatial divergence of the ion beam. The einzel lens consists of three cylindrical stainless steel tubes (3" O.D.), which are mounted concentrically using two cylindrical centering pieces made of DELRIN. This 2 nd region is pumped using a $2 "$ Edwards Diffstak, and typical operating pressures are approximately $2 \times 10^{-7}$ Torr. A $5 \mathrm{~mm}$ ion einzel entrance slit also serves as means of differentially pumping the this region from 
the 1st differential. A pneumatic gate valve located in series with the 2 nd differential is used to isolate the mass spectrometer and source region from the UHV detector region. Located within the second differential region are a series of electrostatic ion optics used to improve or just manipulate the ion beam trajectory, and will be described throughout the sections that follow.

\section{a. Ion Acceleration}

Immediately after the skimmer the ion beam is accelerated coaxially to the desired energy using an ion stack lens. This ion stack consists of 9 concentric and parallel brass rings, whose dimensions are 1.750" 1.D, 2.500" O.D. and are 3/16" thick. Like the skimmer, the first ring electrode is at the float potential $V_{\text {noav }}$ and this voltage is dropped to ground uniformly across the stack; this is accomplished by connecting successive electrodes via $75 \mathrm{~K} \Omega$ resistors. A geometrically varying accelerating field has also been used in our spectrometer, however no change in the ion beam quality was observed.

When coaxial beam acceleration is desired one typically generates the accelerating field using an array of parallel flat grids or thin parallel plates with concentric apertures. To insure that the field equipotentials are flat one usually makes the dimensions of the apertures much smaller than the plate dimensions. There are two main reasons why we have chosen a counter intuitive ion stack geometry are:

a) By using a very large I.D. for the electrodes we avoid collisions between the neutral beam which diverges considerably behind the skimmer, and the electrodes. This minimizes the amount of particles scattering into the ion beam causing it to heat up. In addition, the large open space volume provided by the ring electrode geometry allows 
facile pumping of the region behind the skimmer, thereby maintaining a low chamber pressure; consequently this minimizes the number of collision between the ion beam and the background gas leading to colder ions.

b) The second reason which is less obvious, was observed by Jenny Loeser, and becomes apparent with the aid of fig. 13. In fig. 13(a) shown are the potential contours behind the skimmer, for a geometry where the accelerating field is generated by a series of small apertures; our preferred ring geometry is illustrated in fig. 13(b). The contour and ion beam trajectory simulations were obtained using the SIMION ion optics program. For the case (a) one notes that the potential contours near the first lens are severely curved creating large potential gradients. The energy of the ions emerging from the skimmer is equal to the Maxwell-Boltzmann kinetic energy of the neutral expansion, which is very small, typically about $0.5 \mathrm{eV}$. Hence the ions will follow the potential gradients due the fringe fields just described and undergo a "tight focusing". Focusing an ion beam to a very small volume results in serious repulsion between the ions which produces a highly diverging, non-collimated ion beam (fig. 13, (a)). This situation can be rectified, if one uses the ring electrode geometry of fig. 13(b). Since the potential contours are now relatively "flatter" in the region between the skimmer and the first lens, there is a only mild "focusing effect" on the ion beam, hence allowing the ions to be accelerated with a much smaller diverging effects. To compensate for this ion beam divergence we spatially focus the ions using the decelerating ion einzel lens located in the 2 nd differential region described earlier in this section.

\section{b. Beam Modulation Time of Flight (BMTOF)}


Mass selection of the ion beam is achieved using a collinear time of flight spectrometer developed by Bakker. ${ }^{16}$ The principles of this spectrometer are based on techniques of "ion sweeping", used by nuclear physicists in attempts to generate "short bursts" of ions from long or even continuous ion beams. A theoretical treatment of these "sweeping" techniques has been published by Fowler and Good, ${ }^{17}$ and the authors point out the associated problems and advantages. As Bakker himself points out "sweeping" is something you do with a "broom" and not with individual ions. Instead what beam modulated time of flight does is to create a kink in an ion beam which can then be interrogated and analyzed at some later time, thereby yielding information about the ion beam.

A Bakker type mass spectrometer consists of an electric field whose direction is perpendicular to the ion beam direction, and an exit slit. At some point in time $t_{0}$ the direction of the electric field is reversed very rapidly. Experimentally the electric field could be generated using the two beam modulation plates (BMP) described earlier, and maintaining each plate at a voltage of $+\mathrm{V}_{0}$ and $-\mathrm{V}_{0}$. The electric field direction can be reversed by flipping the polarity of the plates, i.e. modulating the voltage using some waveform. The effects of this voltage modulation can be understood with the aid of fig. 14. Ions that were traversing through the BMP before or after the field modulation will be deflected off axis, thus missing the exit slit (fig. 14, a and c). Ions that were located half way through the plates during the modulation, will be deflected upwards and downwards by the same amount, so the net result will have a negligible effect on their trajectory and will thus make through the exit slit to the detector. 
For voltage modulation waveforms that are very fast, i.e. the rise time of the waveform is much faster then the time it takes the ions to travel through the BMP, the mass resolution of the spectrometer is given by the following equation :

$$
R=\frac{V_{0} \cdot L^{2}}{2 \cdot D \cdot U \cdot(B+S)}
$$

where $U$ and $B$ are the energy and diameter of the ion beam respectively, $S$ the diameter of the exit slit, D is the BMP separation, and $\mathrm{L}$ is the distance between the BMP and the exit slit. Eq. 15 points out some of the advantages of beam modulation time of flight which are:

a) The resolution is independent of the ion mass.

b) The resolution is proportional to the square of the field free drift region, unlike conventional TOF where the relationship between the two quantities is linear.

In a Wiley-McLaren TOF mass spectrometer ${ }^{18}$ an energy spread must be introduced to the ion beam in order to spatially focus the individual ion packets at the detector. Such a procedure is not necessary in BMTOF, and hence it is a very suitable for applications where the energy spread in the ion beam must be small. As will become apparent from the discussion the follows, this would truly be the case if the electric field was modulated in the manner described above. However, this is not a trivial procedure experimentally and so a slightly modified scheme is used.

Shown in fig. 15 is the circuit used to modulate the voltage on the BMP. Using this circuit we initially maintain the plates at a potentials $\mathrm{V}_{0}$ and $\mathrm{V}_{d} / 2$ using a voltage divider. While maintaining one of plates always at $V_{d} / 2$, we reverse the field direction 
by dropping the voltage on the other plate to ground very rapidly $\left(\mathrm{V}_{0} \rightarrow 0\right)$. This occurs when the MOSFET switch goes into conduction. Unlike the ideal situation where one is switching the absolute polarity of the two plates, this procedure introduces an energy spread. This was first observed and explained by Prof. Dan Neumark and becomes apparent with the aid of fig. 16. Consider three ions all of which start out at three distinct potential contours (with respect to ground) shown in fig 16 (top). Following the field modulation the ions end up on potential contours that are different than before , fig. 16 (bottom). We calculate the energy difference between the two situations for the three distinct ions and these are

$$
\begin{aligned}
& \Delta E(A)=0.75 V_{0} \\
& \Delta E(B)=0.50 V_{0} \\
& \Delta E(C)=0.25 V_{0} .
\end{aligned}
$$

It is obvious that the energy gained (or lost) by the three ions is not identical and thus results in a spreading out of the ion packet (poor mass resolution). This energy spread is evidently more pronounced for wide ion beams and can be almost negligible when the ion beam diameter is small.

The energy spread in the ion beam just described, including any others that could have possibly been introduced in the source region during the ion generation, can be corrected (optionally) using a secondary (post-acceleration) acceleration of the individual ion packets (see fig. 16a). This spatial ion focusing is similar to the electron focusing 
described in chapter I (pg.5-6), except that the ions have large kinetic energies compared to the potential across the pulsed field. Rewriting eq. 9 of chapter I for the situation in hand, we find that the flight time $\left(t_{f}\right)$ that it takes the ion packet to reach the focus point is given by the expression

$$
t_{f}=\frac{\sqrt{2 m_{i o n}}}{e . F} \cdot\left\{\left(\sqrt{U_{i o n}+e \cdot S(i o n) \cdot F}-\sqrt{U_{i o n}}\right)+\frac{X_{f} e . F}{2 \cdot \sqrt{U_{i o n}+e \cdot S(i o n) \cdot F}}\right\}
$$

where S(ion) is the position of the ions in the acceleration field, $F$ is the field strength, and $U_{i o n}$ the ion beam energy. We can find the focus point for the ions whose initial positions were $s=s_{0}+\delta s$ by setting $\left(d t_{d} / d s\right)_{50}=0$, and this yields :

$$
X_{f}=\frac{U_{\text {ton }}}{e . F}+S(\text { ion })
$$

For a $1 \mathrm{keV}$ ion beam and a compressing field of $25 \mathrm{~V} / \mathrm{cm}$, eq. 17 estimates that the focus point will be appriximately $40 \mathrm{~cm}$, which agrees well with our experimental observations. In deriving eq. 18 we have assumed that the original ion energy spread from the BMP had manifested itself by spatially spreading out the beam, and that the "correcting" energy spread introduced by this secondary acceleration compensates for this. Without this ion focusing the mass resolution of the spectrometer is approximately 150 , while ion focusing increases the resolution to about 250 . This mass resolution improvement does of coarse come at the expense of narrow energy distribution of the ion packet, and thus the ion focusing is used depending on whether or not the energy or mass resolution is more critical for a particular experiment.

Experimentally the ion focusing (pulsed compression) field is produced by two 
concentric parallel round stainless steel plates, 0.50" I.D., 2.50" O.D.. The plates are mounted perpendicular and symmetrically to the ion beam direction (fig. 12). The plate farthest from the skimmer is maintained at ground potential, while the voltage on the other can pulsed negatively using the circuit of fig. 17. This creates a uniform electric field which accelerates the ions. Since the ion focusing field is located about $60 \mathrm{~cm}$ from the BMP, ions packets of different masses have already been formed; hence the field generation is timed such that the ion packet of interest is half way through the plates.

Often enough, it is desirable to have only ions of some particular mass enter the mass spectrometer region. This is done with an ion gate which consists of a pulsed deflection field mounted immediately after the ion focusing field (see fig. 12). It consists of two parallel stainless steel plates (1"x1", 1/16 thick), mounted parallel to the beam axis. The one plate is maintained at ground and the other pulsed at some voltage, hence creating a deflection field which deflects the undesired ions off axis thereby missing the exit slit. The pulsing circuit used is shown in fig. 19; the deflection field is normally ON until a TTL pulse triggers the MOSFET switch and turns it OFF by grounding the high voltage plate. in our setup, the TTL trigger pulse is the same used to trigger the ion focusing circuit of fig. 17. 


\section{Detector Region}

The detector region is illustrated in fig. 21 , and is the region where the anion of interest is photodetached and analysis of the ejected photoelectrons is performed. It is separated from the 2 nd differential using the $5 \mathrm{~mm}$ exit slit of the mass spectrometer, and is pumped using an Alcatel turbomolecular pump (TMP), $450 \mathrm{l} / \mathrm{sec}$, backed by a Varian TMP, $80 \mathrm{l} / \mathrm{sec}$, and is maintained at operating pressures of $2 \times 10^{-9} \mathrm{Torr}$.

Photodetachment of the anions is performed using an excimer pumped tunable dye laser (Lambda Physik EMG 202 and FL 3002); frequency doubling is possible using both KDP and BBO crystals. The laser power is monitored using a Gentec pyroelectric detector.

The theoretical treatment of the principles of our detection scheme have been thoroughly presented in chapter I. Introducing a small time delay after the photodetachment allows the ZEKE electrons to separate out from the energetic ones; a weak extraction field then allows us to extract and detect only ZEKE (threshold) photoelectrons as a function of wavelength. Since we are photodetaching a fast ion beam (500 or $1000 \mathrm{eV}$ ) the ZEKE electrons produced will be travelling with the same velocity as the ions (typically $5 \times 10^{6} \mathrm{~cm} / \mathrm{sec}$ ), and hence move a certain distance during the time delay as described in chapter I (pg. 3-5, eq. 6). Thus it is important that an extended coaxial extraction field be used for the photoelectrons.

The extraction field is produced by a array of 12 parallel molybdenum plates, $0.030^{\prime \prime}$ thick, $2.5 "$ outer diameter, and $0.5 "$ inner diameter, mounted symmetrically about the instrument axis. The first four plates are separated by $10 \mathrm{~mm}$ and are connected using 
$17 \Omega$ resistors so as to produce a uniform electric field parallel to the instruments axis. The first plate is pulsed between -5 and -10 Volts, while the fourth and successive plates, separated by $5 \mathrm{~mm}$ are grounded. These later plates act as a series of apertures used to discriminate against the off axis energetic electrons as described in chapter I. Electrons are photodetached between the first two plates; the inner diameter of the second and third plate are $3 / 4^{\prime \prime}$ in order to minimize the amount of surface area near the interaction region, thereby minimizing the amount of stray fields due to surface inhomogeneities. To further eliminate any surface effects, we apply a thin layer of graphite to the first four plates (AERODAG, made by Acheson co.), which creates a uniform conducting surface. Cleaning of the molybdenum plates is done using the procedure recommended by Rosebury. ${ }^{19}$ Following the last plate, there is a $60 \mathrm{~cm}$ drift region. To improve the ZEKE electron collection efficiency, a deceleiating einzel lens made of three cylindrical and concentric OFHC copper tubes (3.0" O.D.), has been installed in the drift region (fig. 21).

All the photoelectron optics mentioned are mounted inside two coaxial tubes from Hypernom, which is a material with a very large magnetic permeability. This provides magnetic shielding from the earch's magnetic field and is essential to the experiment. The physics behind the magnetic shielding has to do with the boundary condition of the perpendicular component of the magnetic filed at an interface, and the details are worked out in any electromagnetics text book. ${ }^{2}$

A deflection field located immediately after the drift region is used to deflect photoelectrons onto the electron detector, a Chevron-mounted pair of $45 \mathrm{~mm}$ micro- 
channel plates (Galileo) mounted perpendicular to the heam axis. The electron detector circuitry is shown in fig. 22. The deflection field voltage can be varied in order to ensure that all the deflected electrons are detected. Since the channel plates are at high voltages it is extremely important to shield the electron trajectory from this voltage! This is achieved by using a grounded solid sheet of stainless steel as shown in fig. 22 .

The ion detector is shown in fig. 23 and is a Chevron-mounted pair of microchannel plates (Galileo) located at the end of the machine. the grid in front of the detector is at ground when ions are detected, and can be biased to some negative voltage thereby repelling the anions and allowing only neutrals, generated during the photodetachment to be detected.

\section{Timing and Data Acquisition}

A diagramm of the data acquisition electronics is shown in fig. 18. All the timing in this experiment is controlled with a Stanford Research Systems DG 535 delay generator and several home built delay circuits. The timing sequence is :

Pulsed Valve $(\mathrm{T}) \rightarrow$ Vaporization Laser $(\mathrm{A}) \rightarrow \mathrm{BMP}(\mathrm{B}) \rightarrow$ Ion Focus and Gate $\rightarrow$ Photodetachment Laser (C) $\rightarrow$ Electron Extraction (D).

The ion signal, the laser power, and the preamplified (Ortec 9301) electron signal, are integrated by a LeCroy $2249 \mathrm{SG}$ gated A/D converter. All trigger pulses and analog signals going to the $\mathrm{A} / \mathrm{D}$ converter must be negative $(\geq-1.4 \mathrm{~V})$ and terminated to $50 \Omega$. The delays and widths of the gates used by this integrator are produced by the circuits shown in figs. 24a, 24b, 24c, and were designed and build by Jenny Loeser and Prof. Irene Waller. For the electron signal, the detection gate width can be adjusted during the 
experiment in order to achieve the maximum discrimination bstween ZEKE and energetic electrons. The information from the A/D converter (ion signal, laser power, and electron signal) is transferred through a DSP 6001 CAMAC controller to a personal computer where it is stored after each laser shot. The electron signal is normalized to the laser power and the ion signal. The repetition rate of the experiment is $20 \mathrm{~Hz}$ when the Nd:YAG laser is used for laser vaporization, and $50 \mathrm{~Hz}$ otherwise. The software for running the experiment and handselling the data were written by Jenny Loeser.

\section{Vacuum Interlock System}

If the pressure in any part of the spectrometer begins to rise above the safe operating limit, a home built interlock system which will take the appropriate measures in order to prevent any irreversible damage to the spectrometer. This interlock system is similar to the one build by Ricardo Metz for the photoelectron spectrometer operating in our laboratory. The circuit diagram is shown in fig. 25 and when set on auto-response its basic functions are:

a) If the foreline pressure of either the source or the first differential pressure rise above 0.1 Torr, the gate valve will shut, the bell will sound and the corresponding diffstack will turn off. In addition the float and detector (both ion and electron) power supplies will turn off. The "tripping" pressures can be set to any desired setting.

b) If the ionization gauge which is used to measure the pressure of the second differential turns off, the gate valve will shut and the bell will ring, the float and detector (both ion and electron) power supplies will turn off.

c) If a diffstack overheats it will turn itself off, the bell will sound, the gate valve 
will shut, the float and detector (both ion and electron) power supplies will turn off. Since the interlocks of the 6" and $2 "$ diffstacks are connected in series, if one fails the other will also. The reason we connected them in series was because the 2 " diffstack was a late addition to the instrument, and since the interlock systern was already installed, this was the most convenient solution. However to our knowledge, there is no disadvantage to having them in series.

d) If the detector foreline pressure rises higher then 0.1 Torr or the detector ionization gauge tums off, the gate valve will shut, the bell will sound, the TMP's will tum off, float, the ion and electron detector power supplies will tum off, and the detector chamber will vent to atmospheric pressure using nitrogen (or any desired gas that one hooks up to the vent valve). There is a manual valve in line with the solenoid vent valve which will prevent venting if it is shut.

c) Interlock bypassing is possible with the Bypass and Rough switches. When the Bypass switch is activated, then the situation a,b,c mentioned above are ineffective, while $d$ is still effective. When the Rough switch is active then situations $a, b, c$ are effective and $\mathrm{d}$ is ineffective.

\section{Photodetachment of $\mathrm{I}^{-}$}

$1^{-}$was chosen as the first test of the instrument. The electron binding energy of I- was previously determined to be $3.0591 \mathrm{eV}(405.3 \mathrm{~nm})$ by laser optogalvanic spectroscopy. ${ }^{20}$

$\mathrm{I}^{-}$was prepared by electron bombardmeni of a dilute mixture of $5 \% \mathrm{CF}_{3} \mathrm{l} / \mathrm{He} \mathrm{mix}$, expanded through our pulsed molecular beam; backing pressures of 20 to $40 \mathrm{psig}$ and 
a 0.030 " nozzle diameter were used and I- was formed by dissociative attachment (chap. II, sec. 2b). In fig. 26 , the threshold photodetachment spectrum we obtained for $\mathrm{I}^{-}$is presented. The peak in the spectrum is from the transition

$$
I-\left({ }^{1} S_{0}\right)+h v \rightarrow I\left({ }^{2} P_{3 / 2}\right)+e^{-} .
$$

Data were taken at $0.025 \mathrm{~nm}$ intervals, and the (FWFM) width of the peak is $3 \mathrm{~cm}^{-1}$. The peak rises sharply at the threshold for this transition and falls off more slowly to the lower wavelength. This "saw-tooth" peak shape results from the discrimination against the high energy electrons and is typical of the thresholds observed with this spectrometer.

\section{Photodetachment of $\mathrm{SH}^{-}$}

As a more interesting test of the capabilities of our instrument, we have measured the threshold photodetachment spectrum of $\mathrm{SH}^{-}$. Both $\mathrm{SH}$ and its anion are well characterized. ${ }^{21.22}$ The neutral and the anion have similar bond lengths, indicating that a $\Delta v=0$ transition will occur upon photodetachment of the anion to the ground state of SH. Sir.ce the rotational constants for both the neutral and the anion are fairly large, approximately $9.4 \mathrm{~cm}^{-1}$, it should be possible to resolve individual rotational transitions with our spectrometer.

The absolute photodetachment cross section for $\mathrm{SH}^{-}$was first measured by Steiner. ${ }^{23}$ Higher resolution measurements in an ion cyclotron resonance cell by Janousek and Brauman ${ }^{24}$, and more recently by Larson ${ }^{25}$, showed rotational transitions appearing as changes in the slope of the cross section. Breyer et al. ${ }^{26}$ studied $\mathrm{SH}^{-}$with fixed frequency photoelectron spectroscopy. At a resolution of 5-7 meV, they were able to observe rotational contours but no individual rotational transitions. 
In fig. 27 a rotational energy level diagram for the $\mathrm{SH}^{-}$, $\mathrm{SH}$ system is presented. Since the ejected photoelectron has spin $1 / 2$, the selection rules for each ion $\rightarrow$ neutral transition ar $\Delta J= \pm 3 / 2, \pm 1 / 2$, and there are hence a maximum of four possible transitions from a given rotational level in the anion to each spin-orbit state of the neutral..$^{25,27}$ For similar rotational constants in the anion and the neutral, transitions in the $\Delta J= \pm 1 / 2$ branches should be separated approximately by $B$, and those in the $\Delta J= \pm 3 / 2$ branches by $3 \mathrm{~B}$.

$\mathrm{SH}^{-}$was also prepared by electron bombardment of a free jet expansion of a $5 \%$ $\mathrm{H}_{2} \mathrm{~S} / 15 \% \mathrm{NF}_{3} / \mathrm{He}$ mixture. $\mathrm{F}^{-}$is produced by dissociative attachment of slow electron to $\mathrm{NF}_{3}$, which then yields $\mathrm{SH}^{-}$via the reaction :

$$
\mathrm{F}^{-}+\mathrm{H}_{2} \mathrm{~S} \rightarrow \mathrm{SH}^{-}+\mathrm{HF} \text {. }
$$

Figures $28 \mathrm{a}$ and $28 \mathrm{~b}$ show the threshold spectra for transitions to the $\mathrm{SH}\left({ }^{2} \Pi_{3 / 2}\right)$ ground state and the $\mathrm{SH}\left({ }^{2} \Pi_{1 / 2}\right)$ excited spin orbit multiplet, respectively. Individual rotational transitions are resolved, and the peak assignments are indicated in the figure. This is the first observation of clearly resolved rotational peaks in the direct photodetachment of a negative ion.

The intensities in both branches can be modelled fairly well by assuming a rotational Boltzmann temperature of $50.75 \mathrm{~K}_{\text {for }} \mathrm{SH}^{-}$. The apparent temperature is very sensitive to the expansion conditions, but we are certainly obtaining cooler ions than any other method used to study $\mathrm{SH}^{-}$to date. Several recent studies of ions and ion clusters have used an ion source similar to the one used here. Our results demonstrate that one can in fact obtain cold aniuns from this type of source even after skimming the beam and 
subsequent acceleration fairly close to the beam valve.

\section{Conclusion}

We have demonstrated the operation of a threshold photodetachment spectrometer with $3 \mathrm{~cm}^{-1}$ resolution. This instrument has been used to obtain a rotationally resolved photodetachment spectrum of a negative ion, $\mathrm{SH}^{-}$. In the chapters that follow we will apply this instrument to the study of the transition state in chemical reactions via negative ion photodetachment and the spectroscopy of mass selected carbon and silicon clusters. 


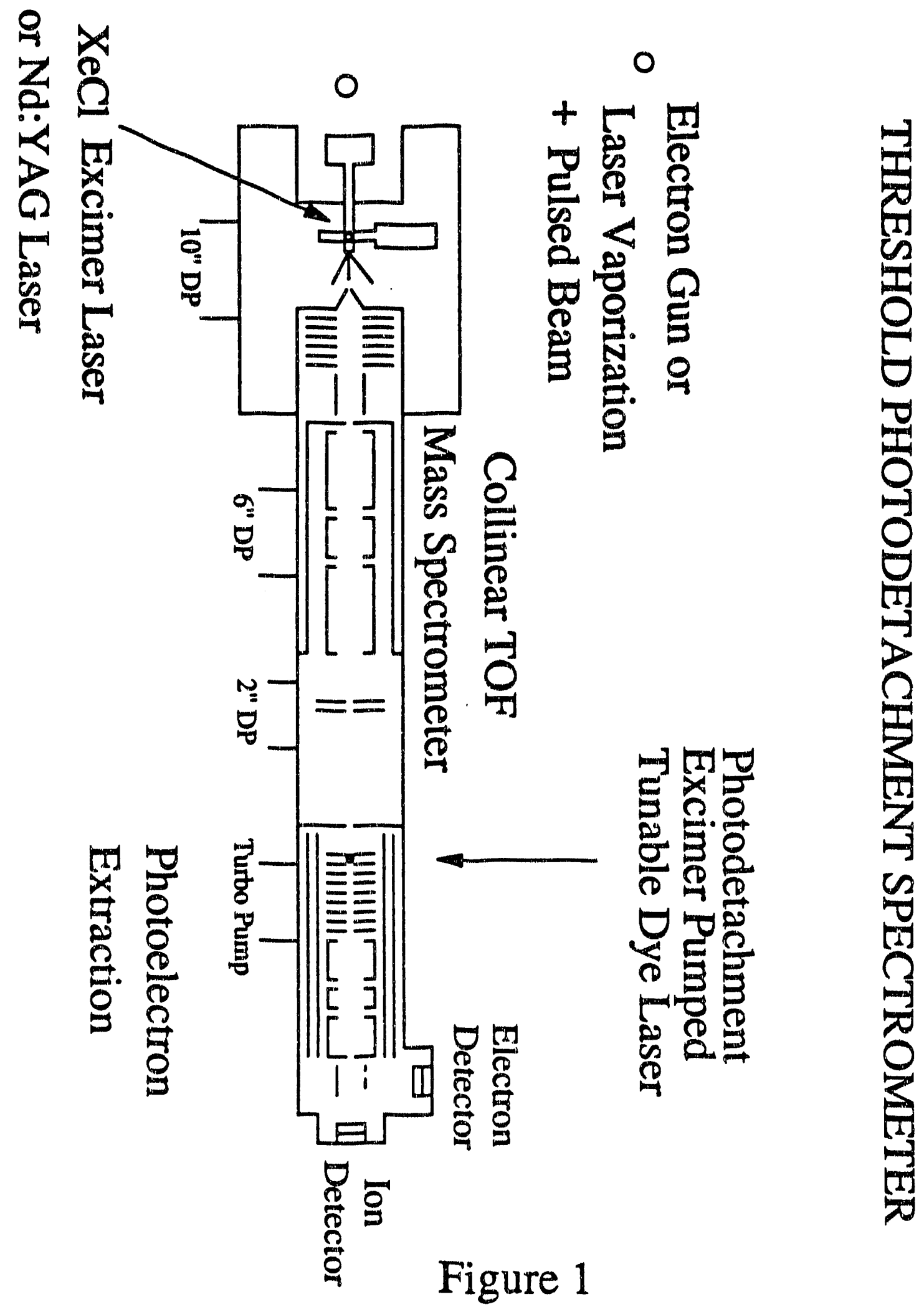




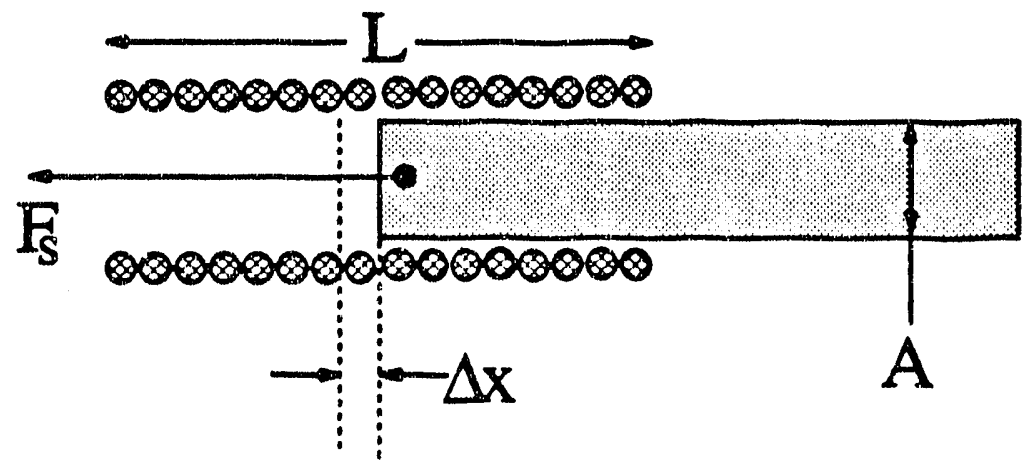

Figure 2 


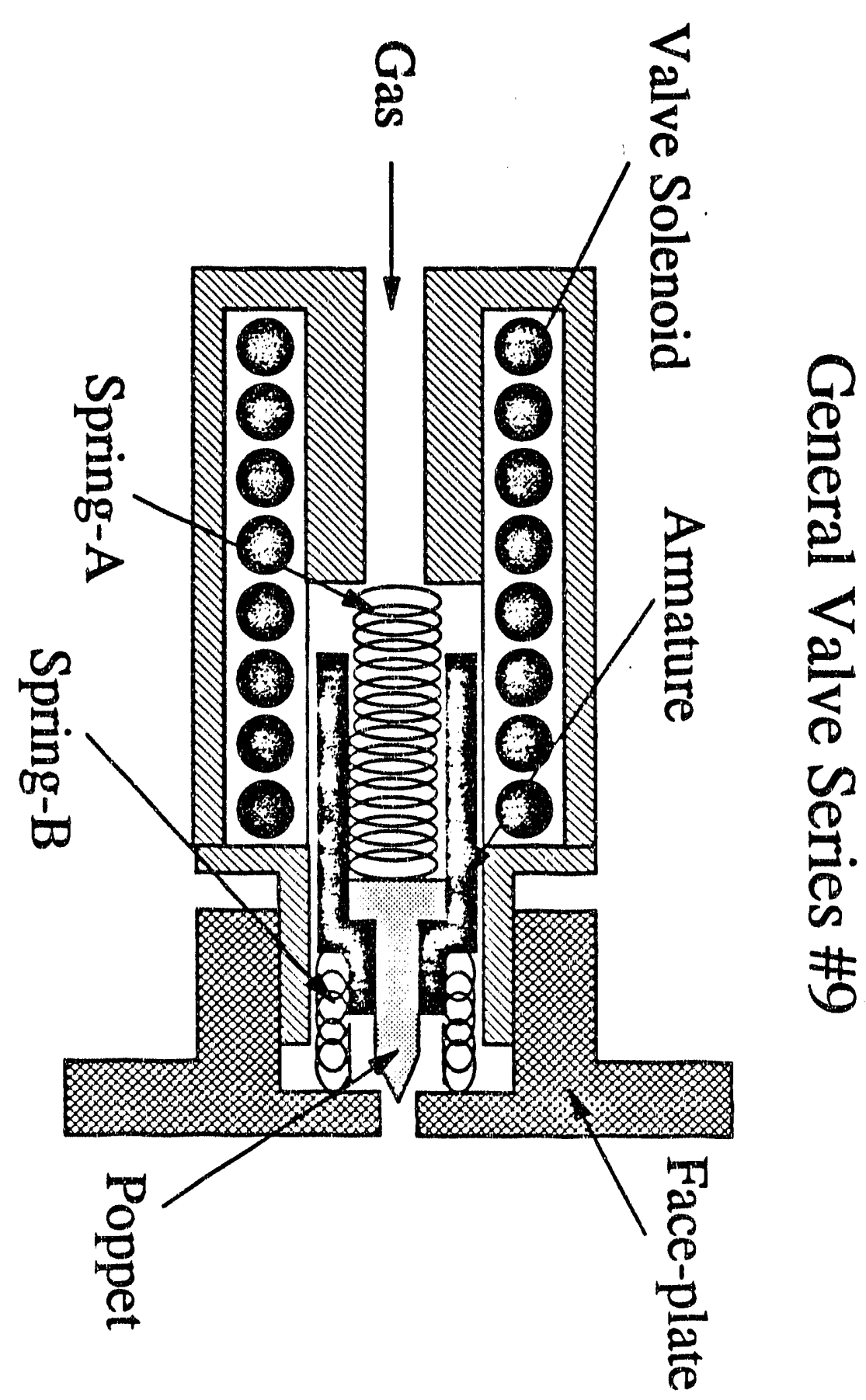

Figure 3 


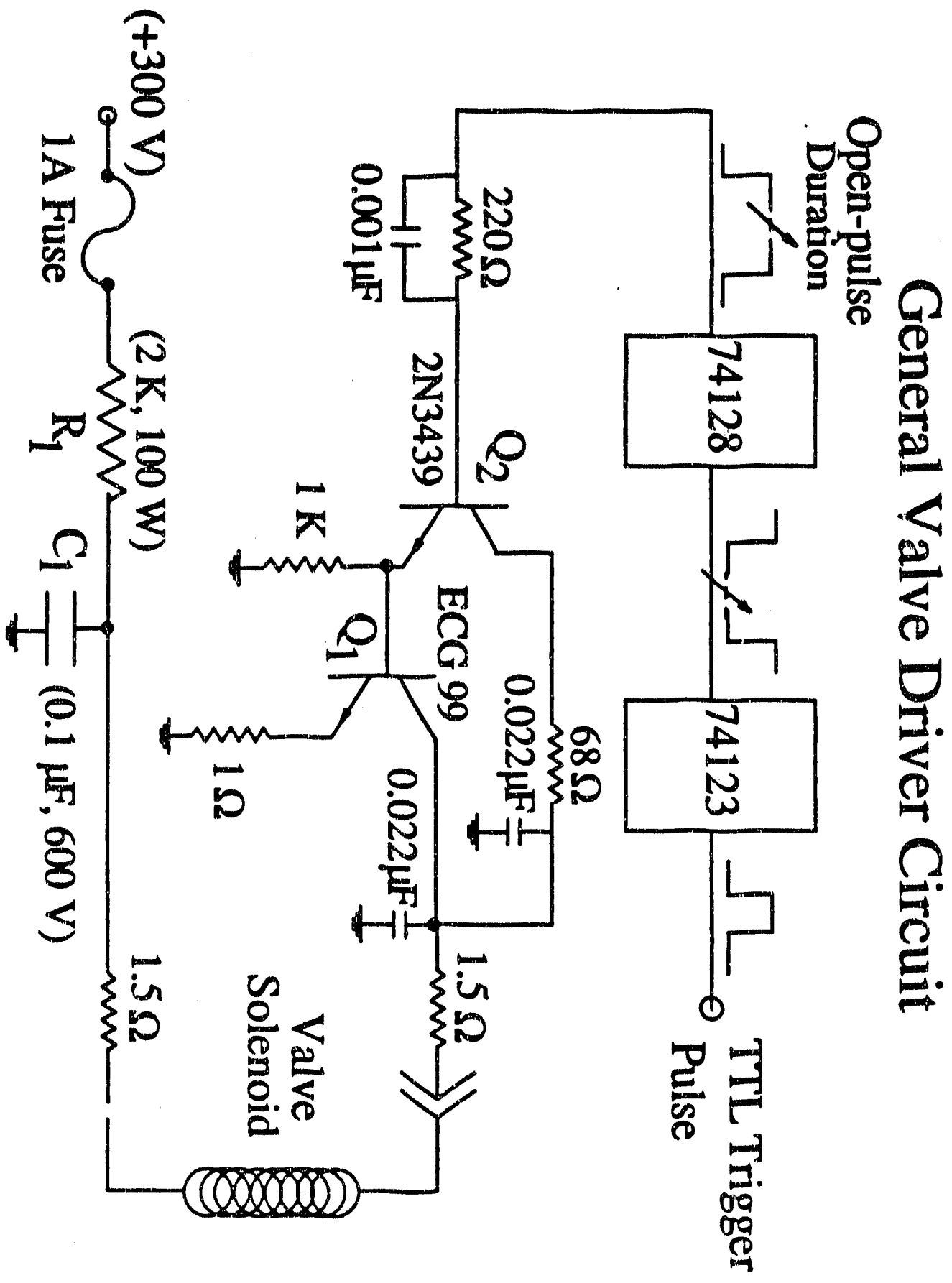

Figure 4 


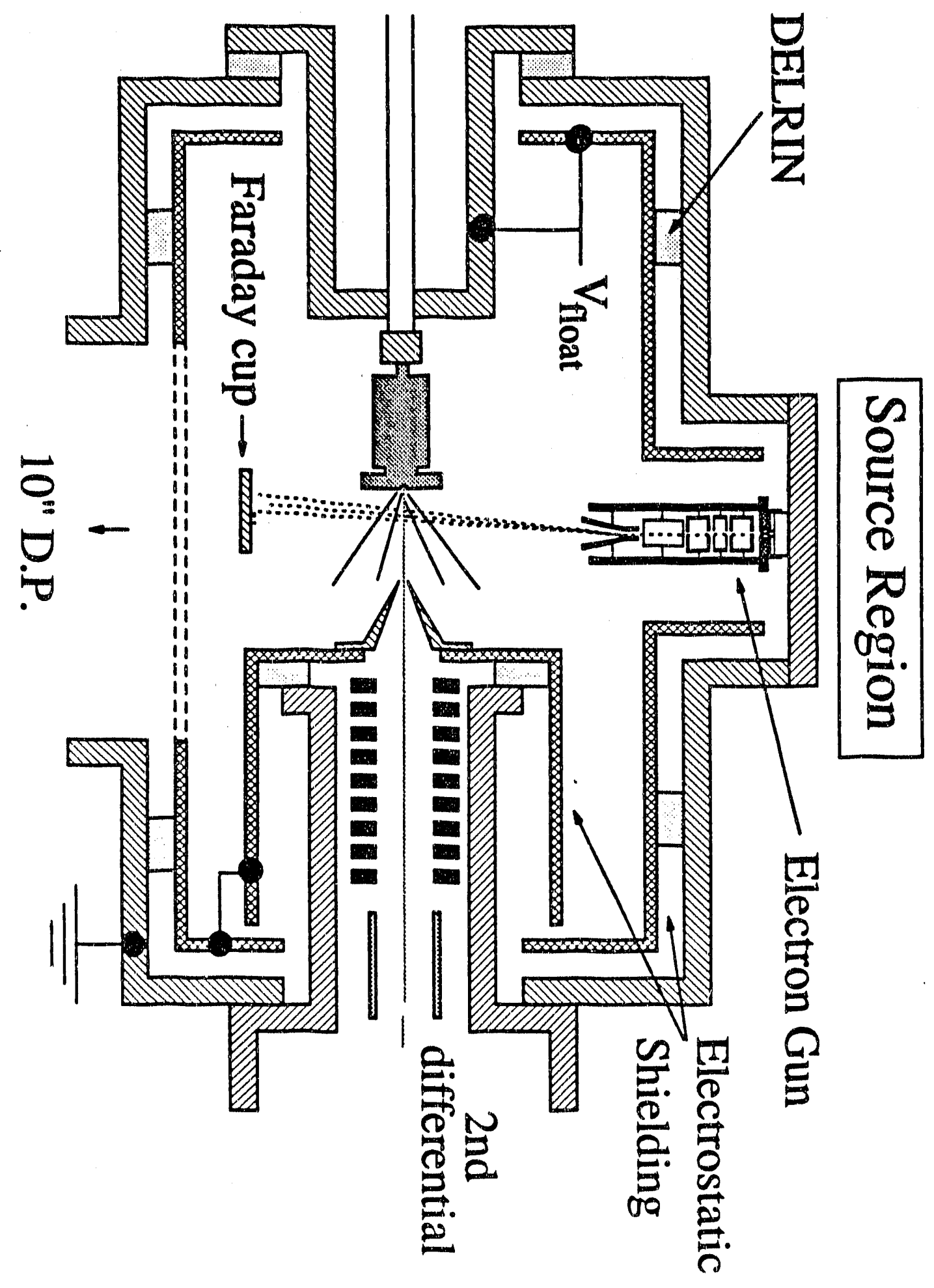

Figure 5 
Electron Gun

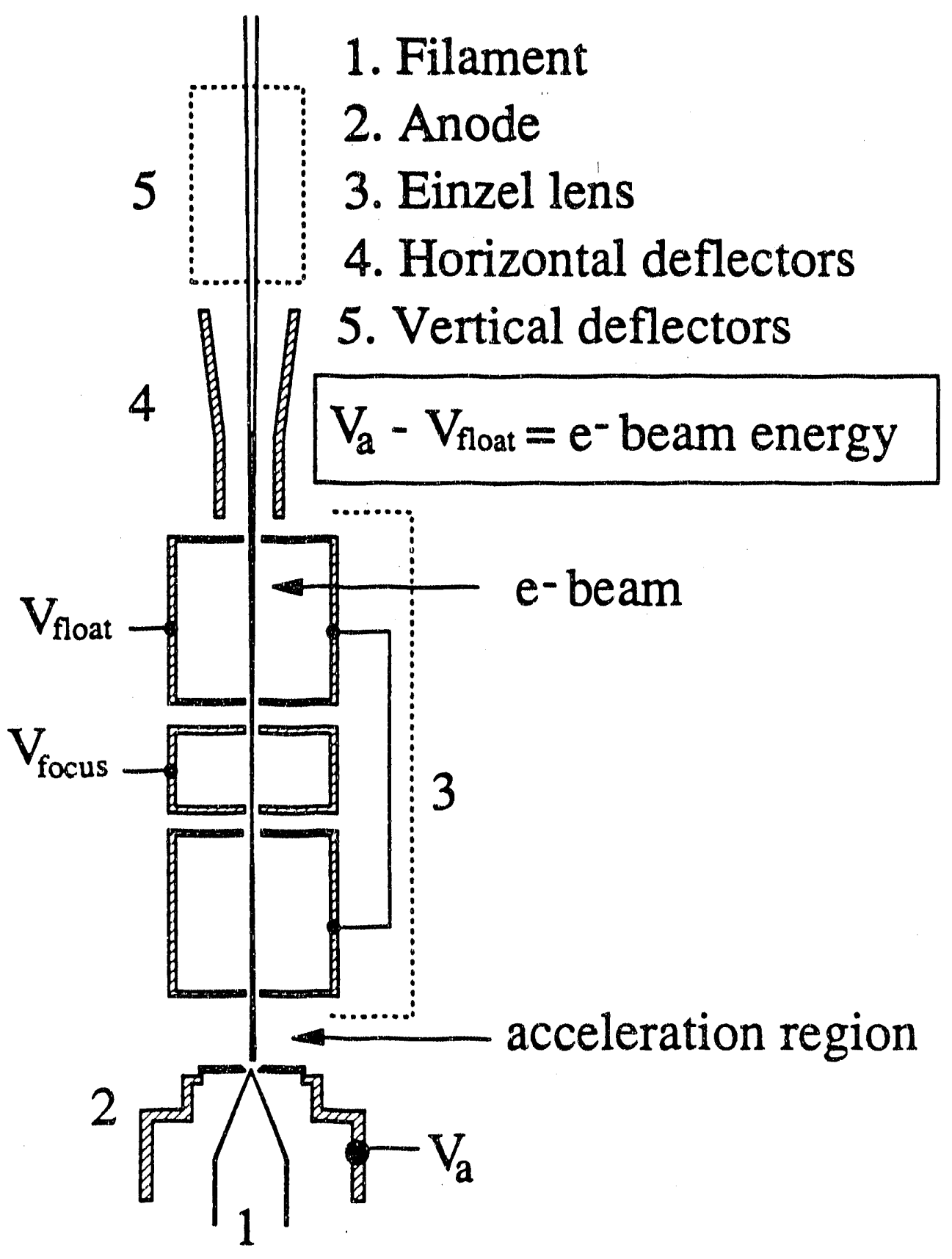

Figure 6 

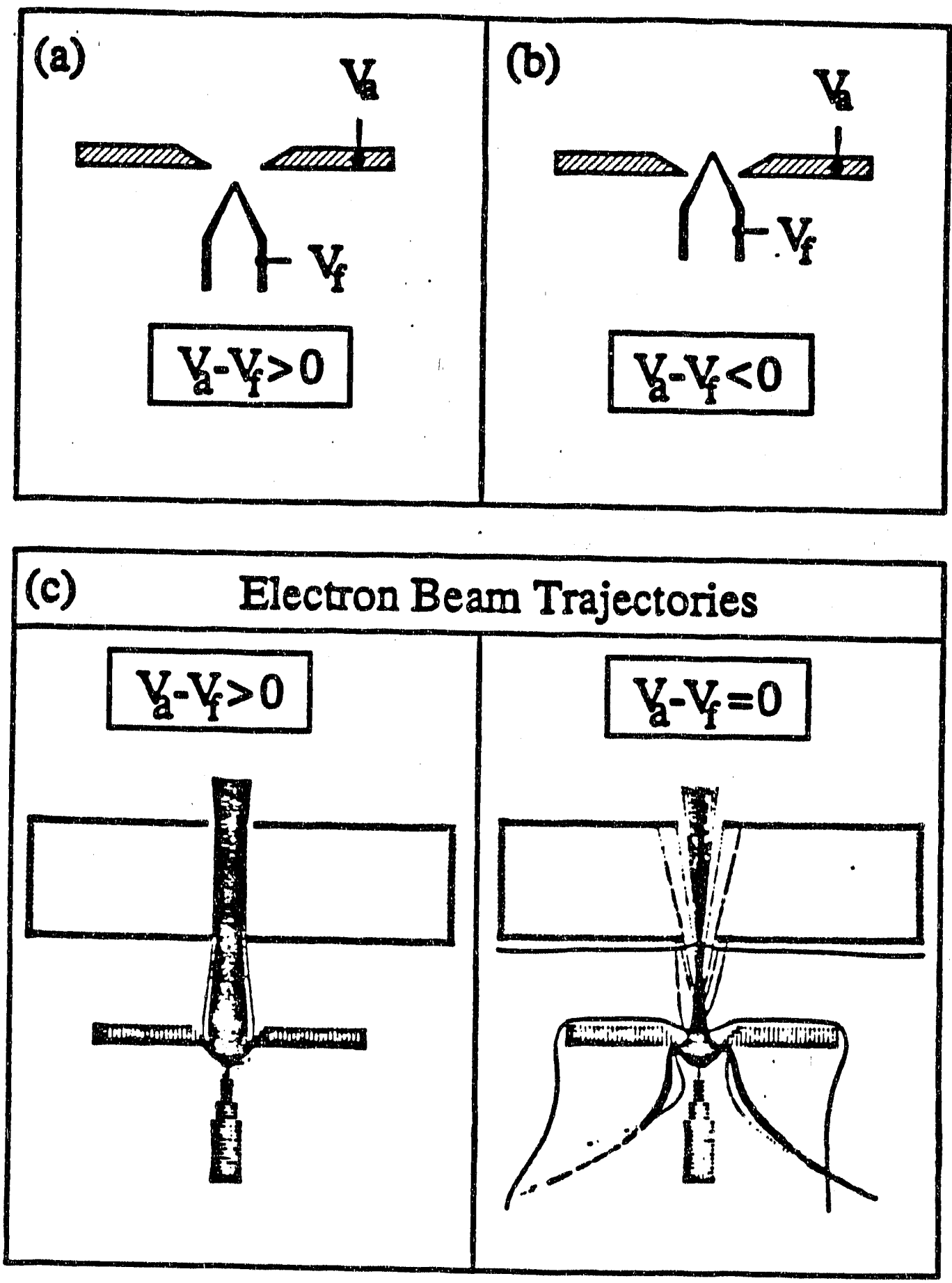

Figure 7 


\section{Isolation Transformer}

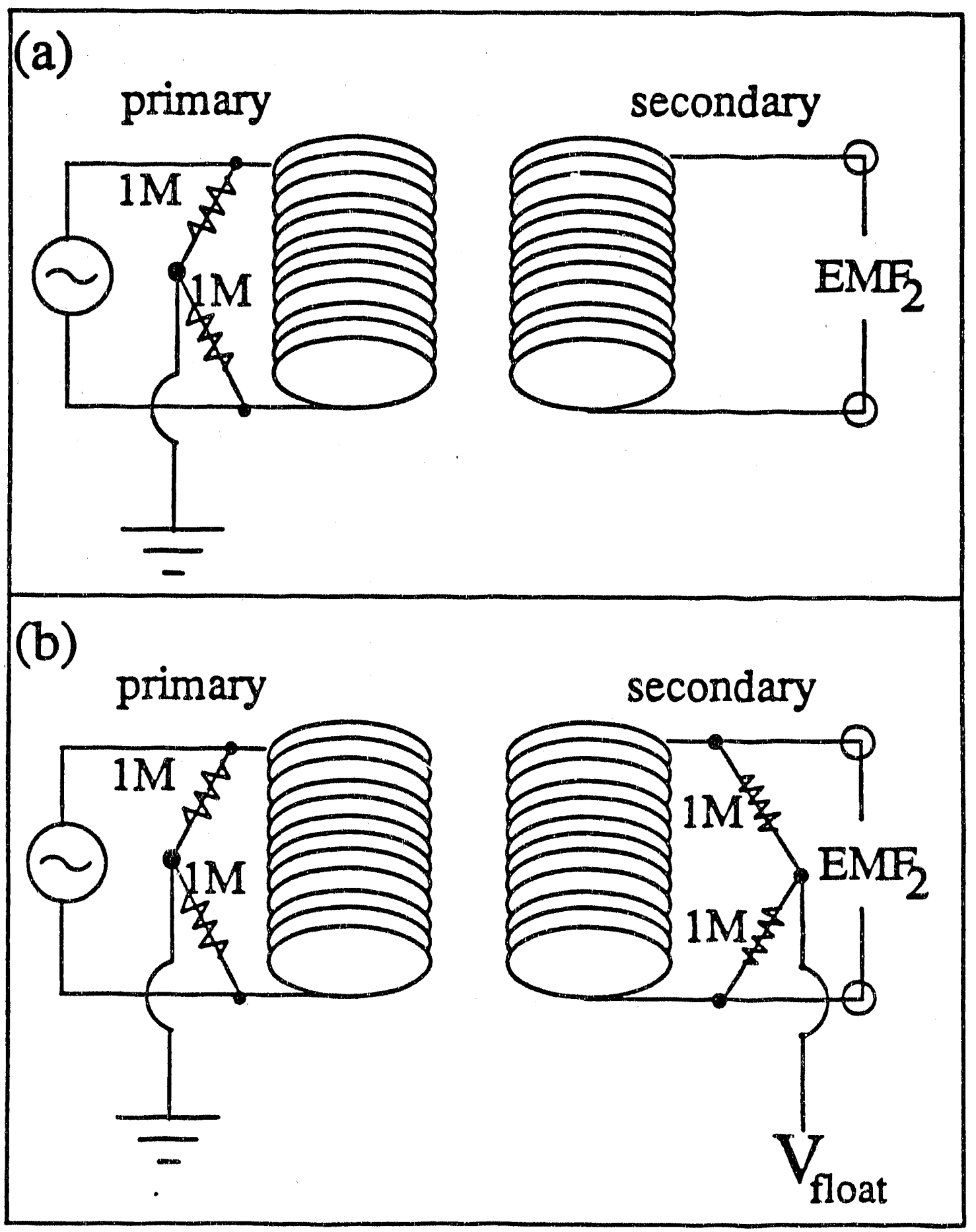

Figure 8 


\section{Source Floating Scheme}

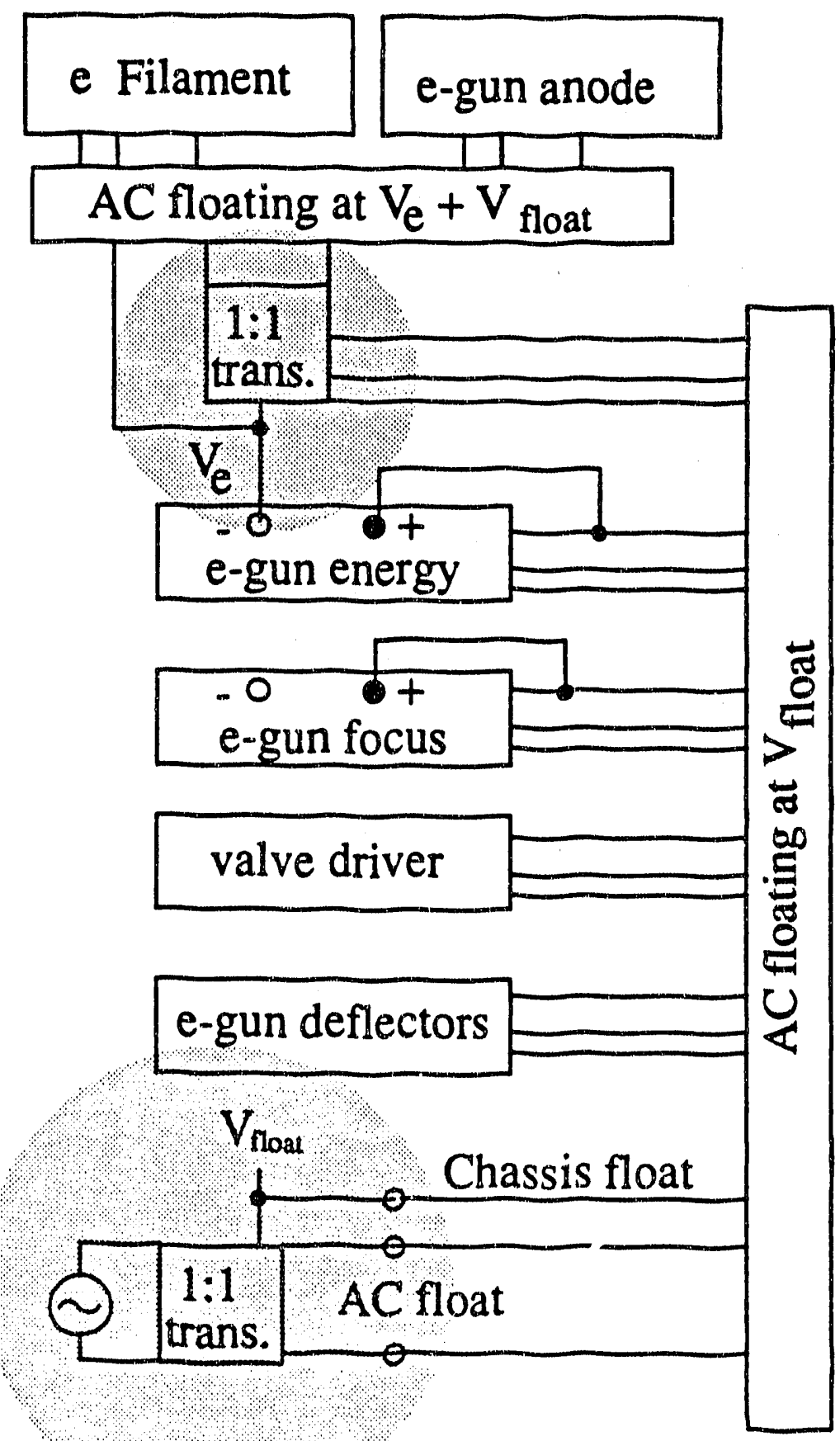

Figure 9 


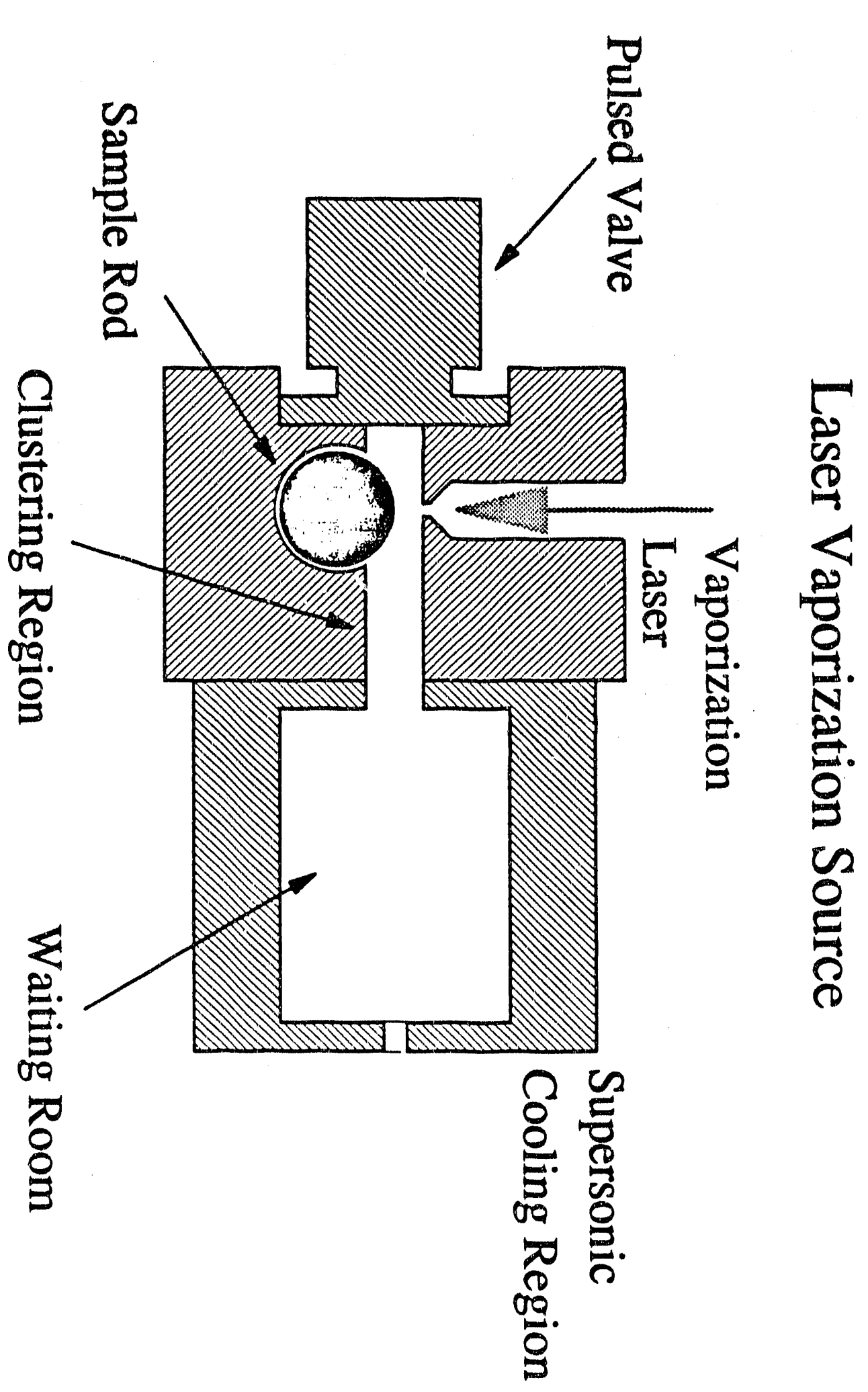

Figure 10 


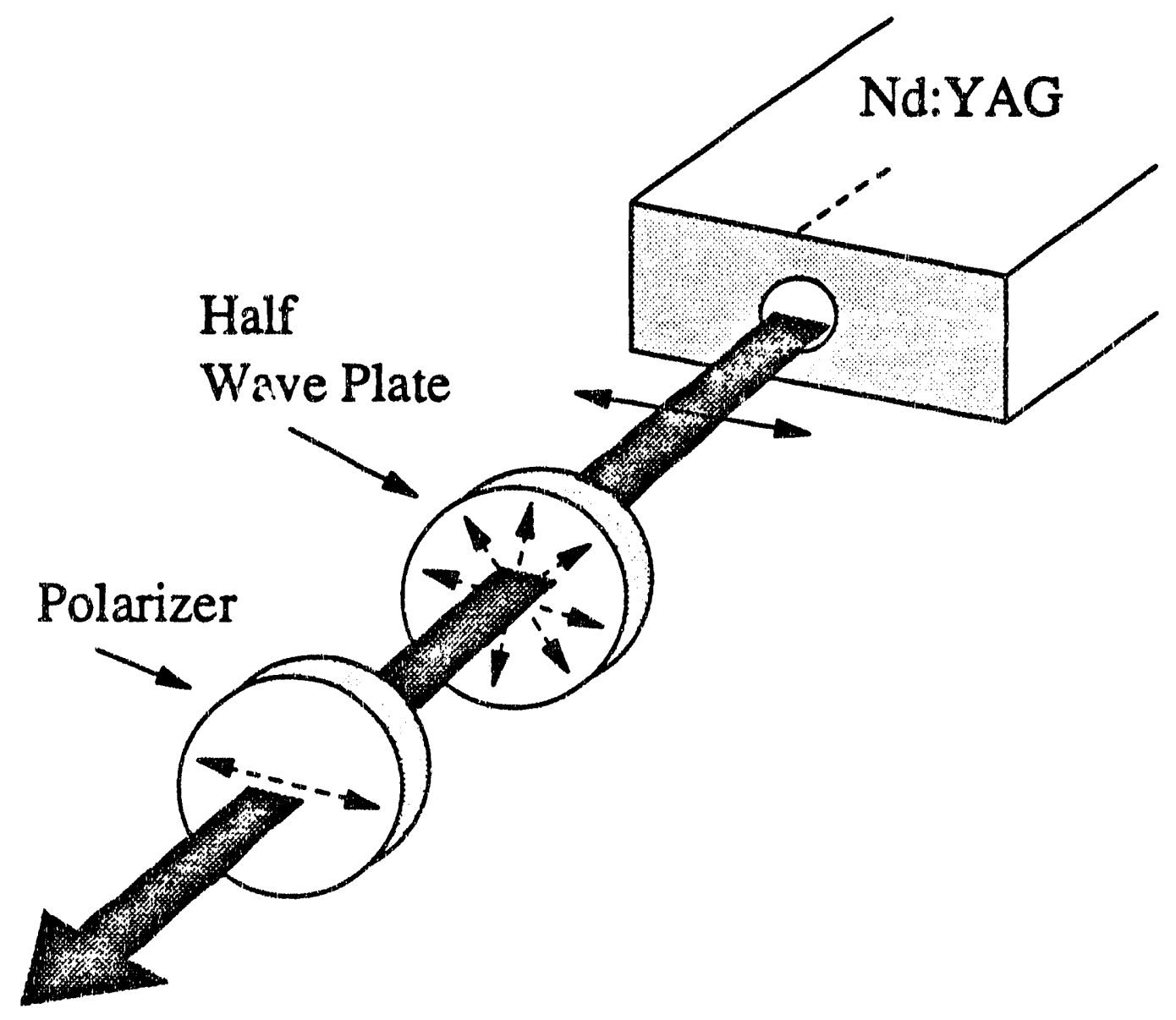

Figure 11 


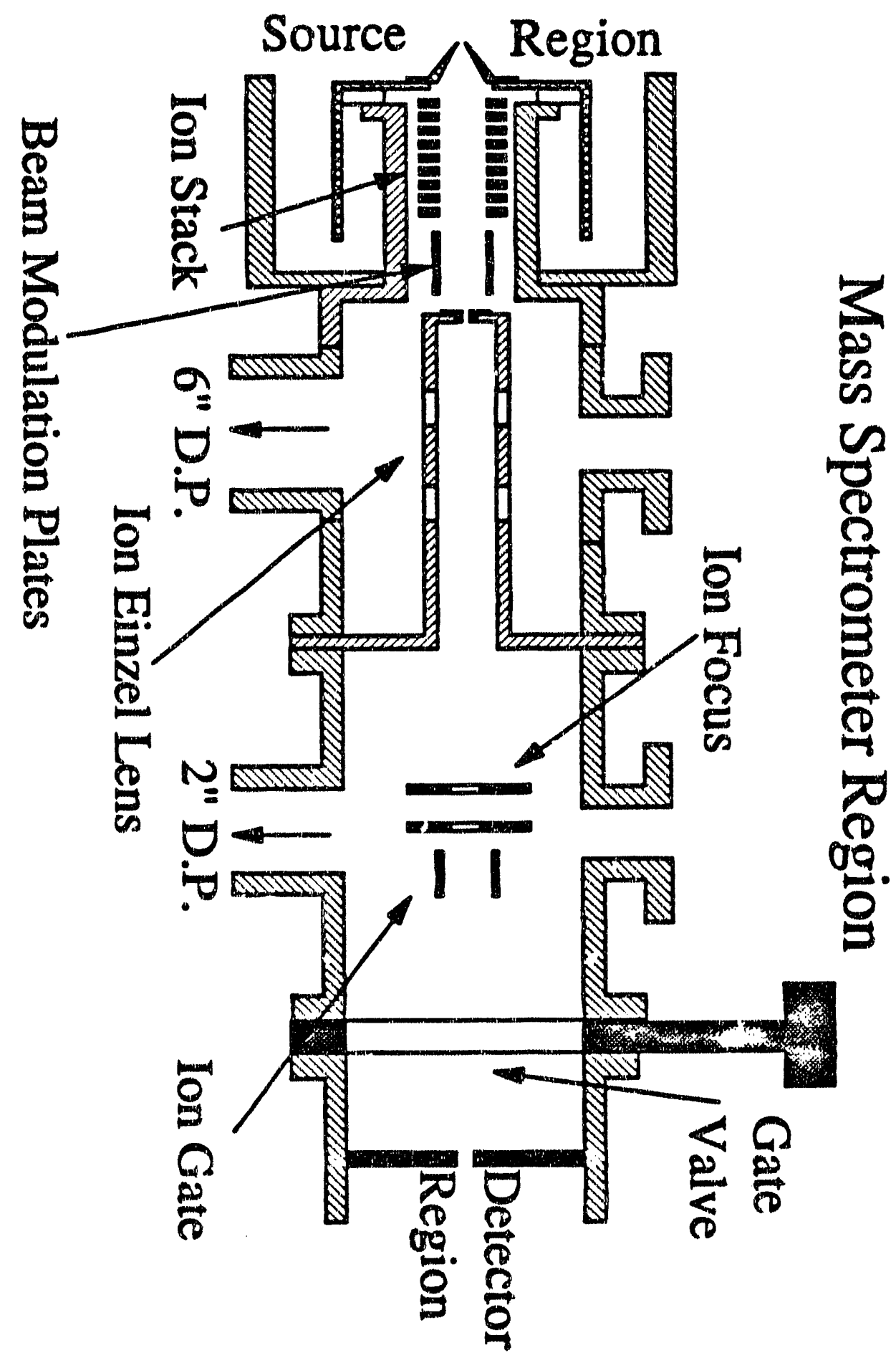

Figure 12 


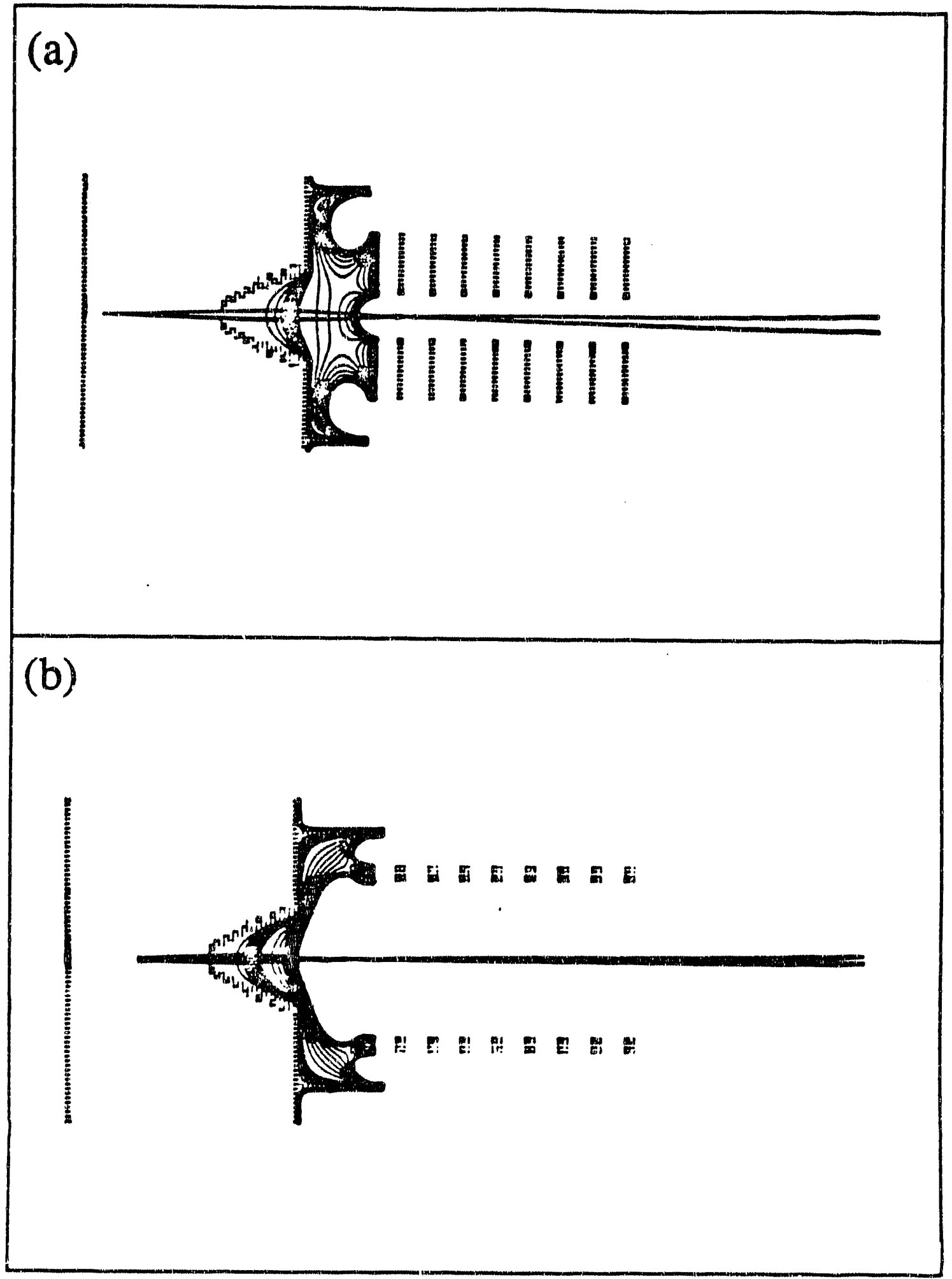

Figure 13 


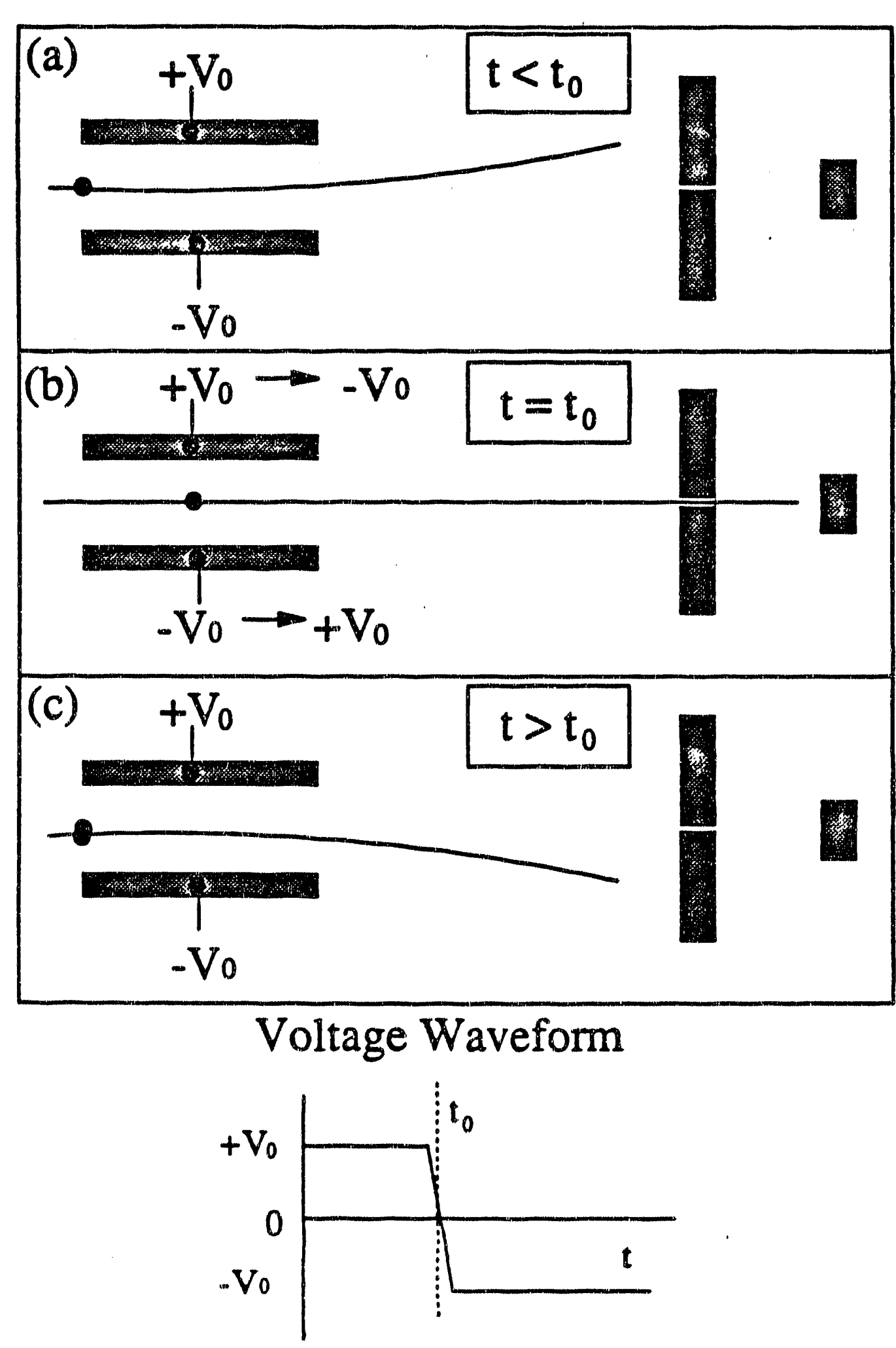

Figure 14 


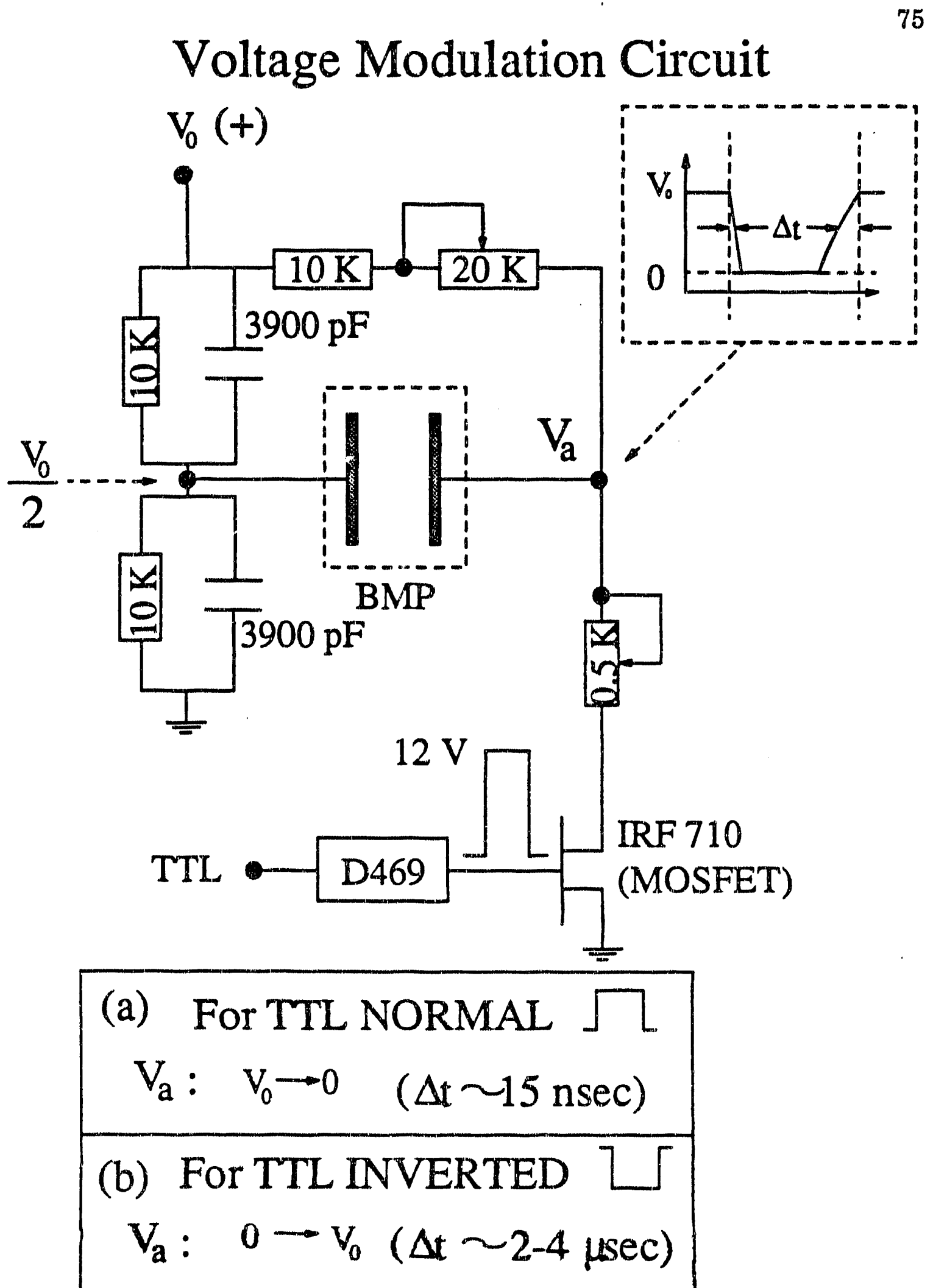

Figure 15 

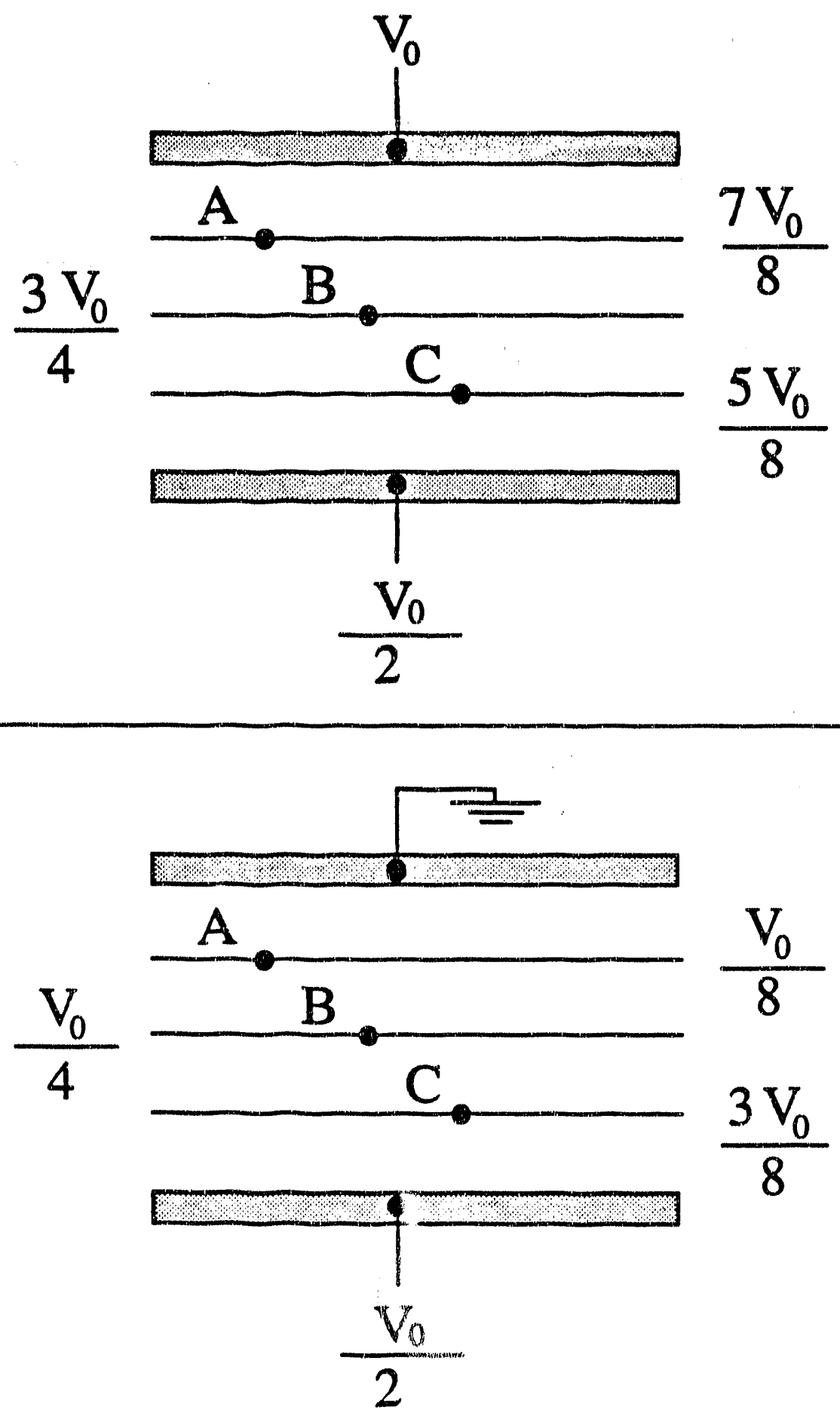

Figure 16 


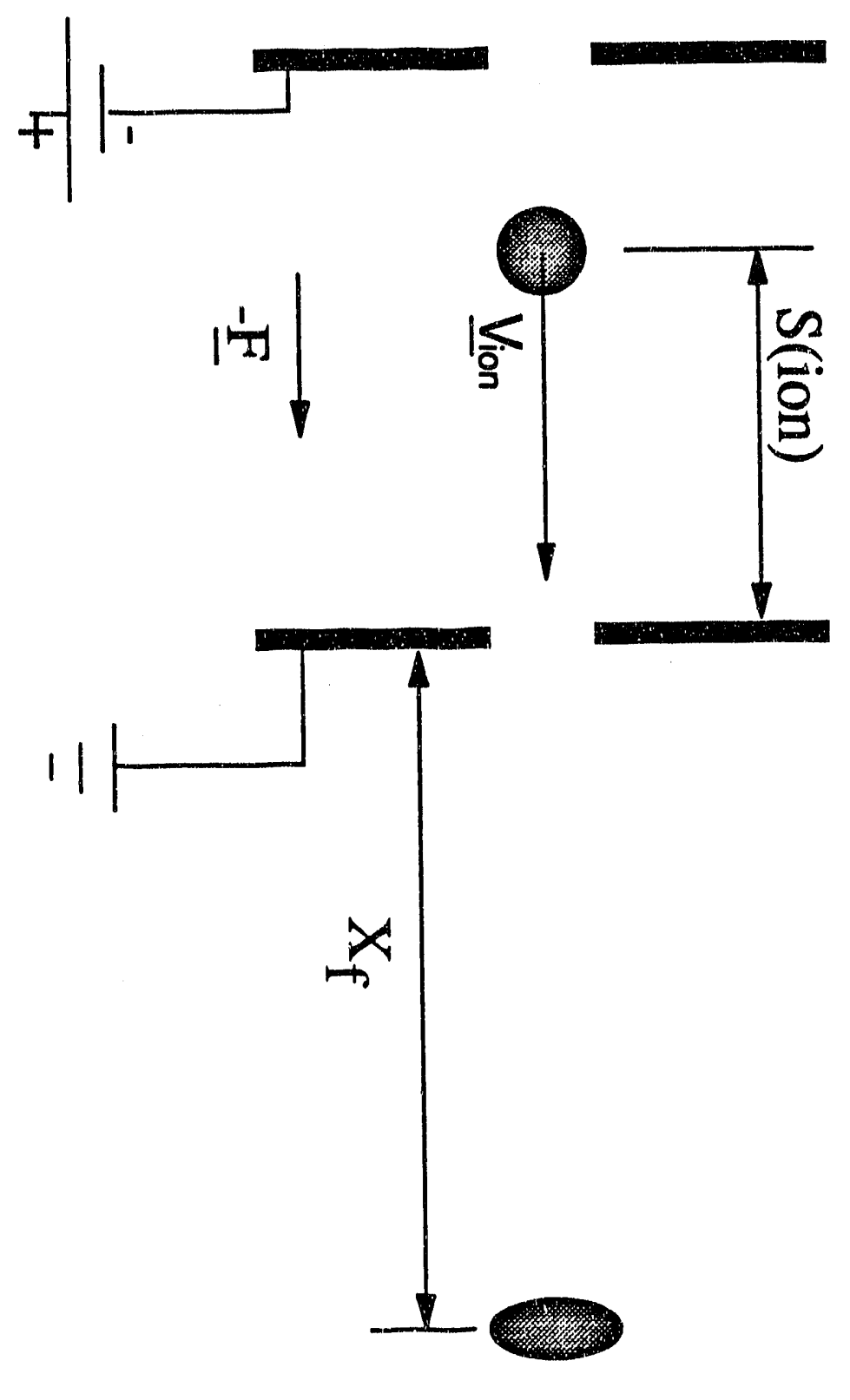

Figure 16a 


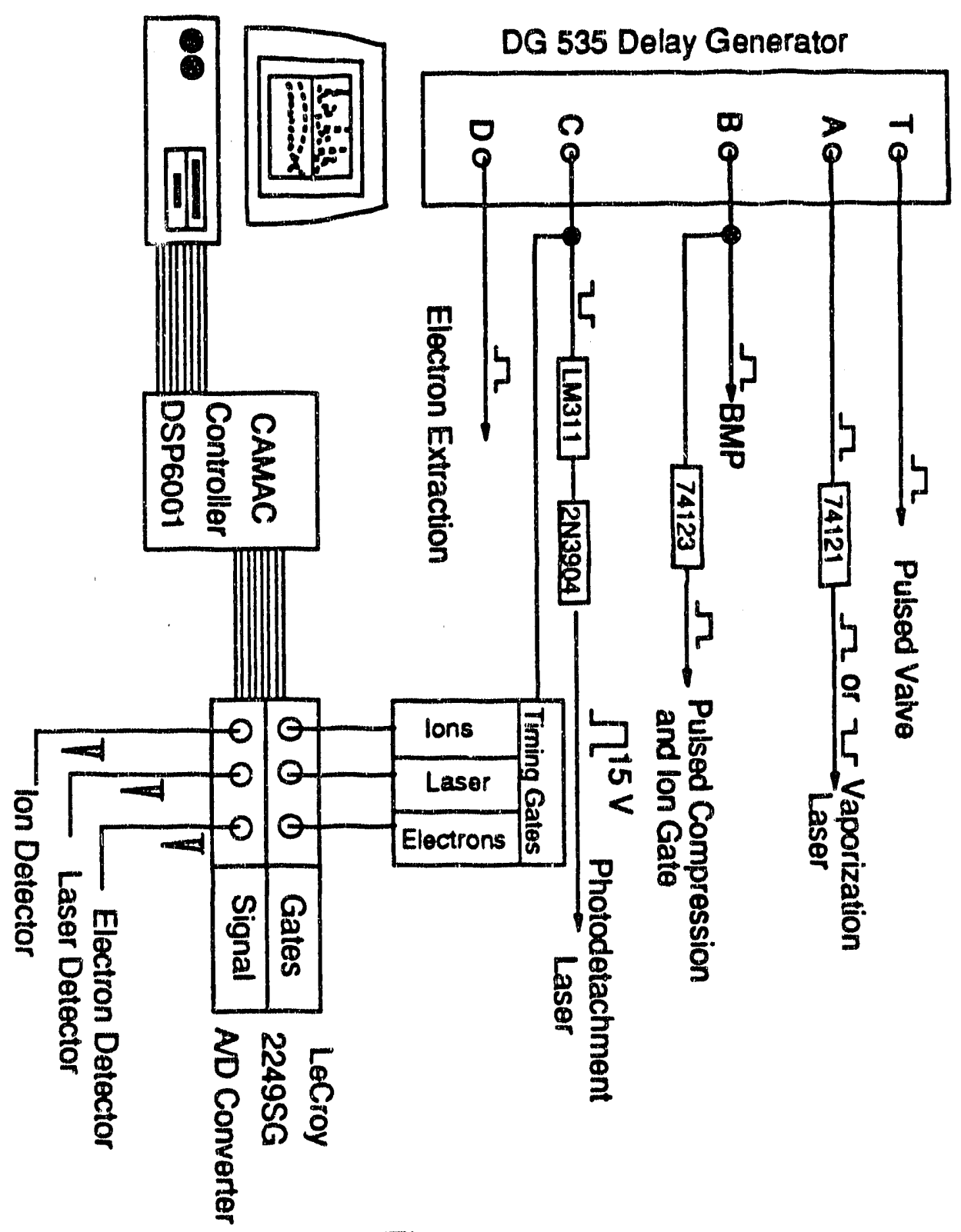

Figure 18 
Ion Gate Circuit

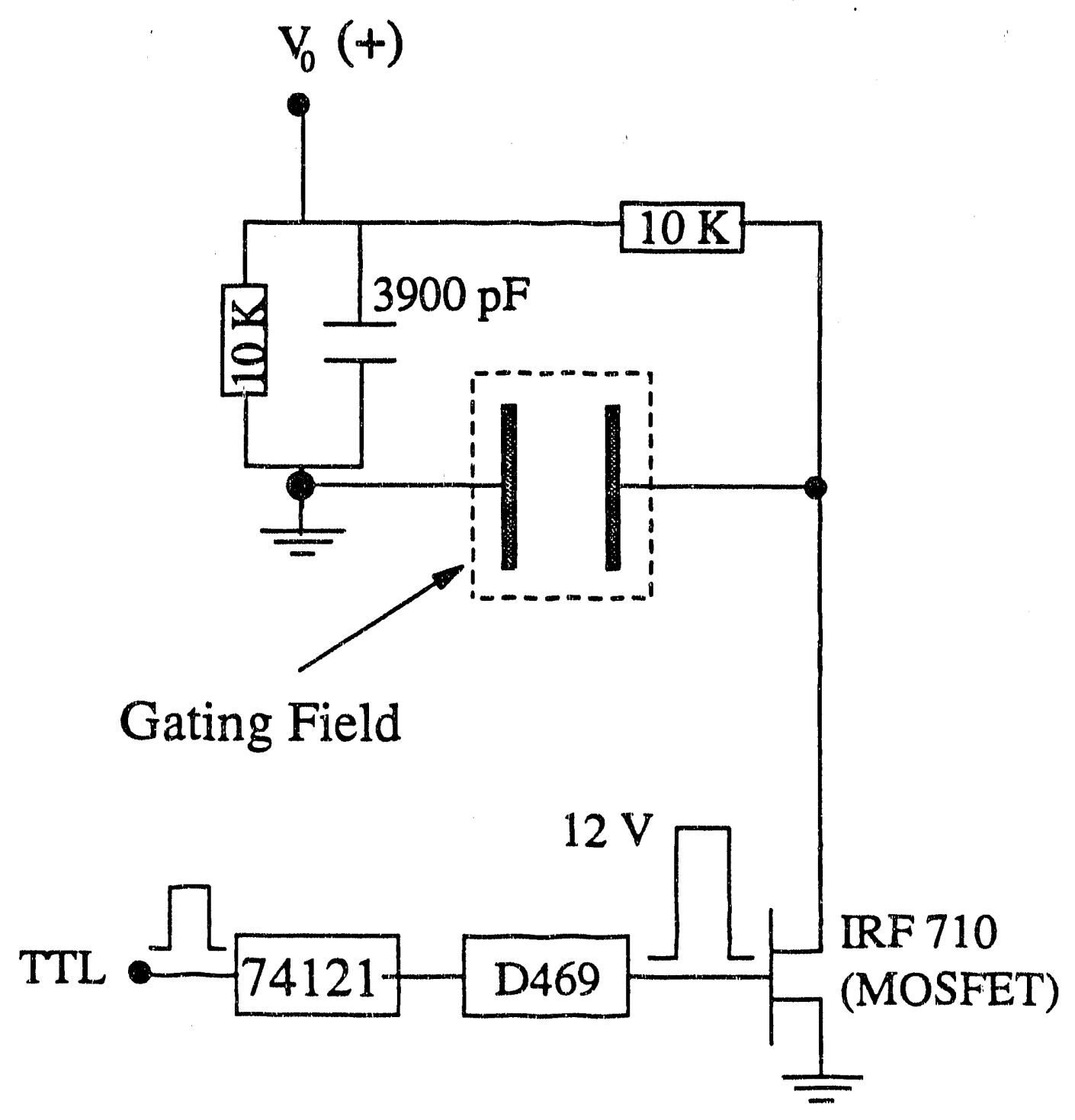

Figure 19 


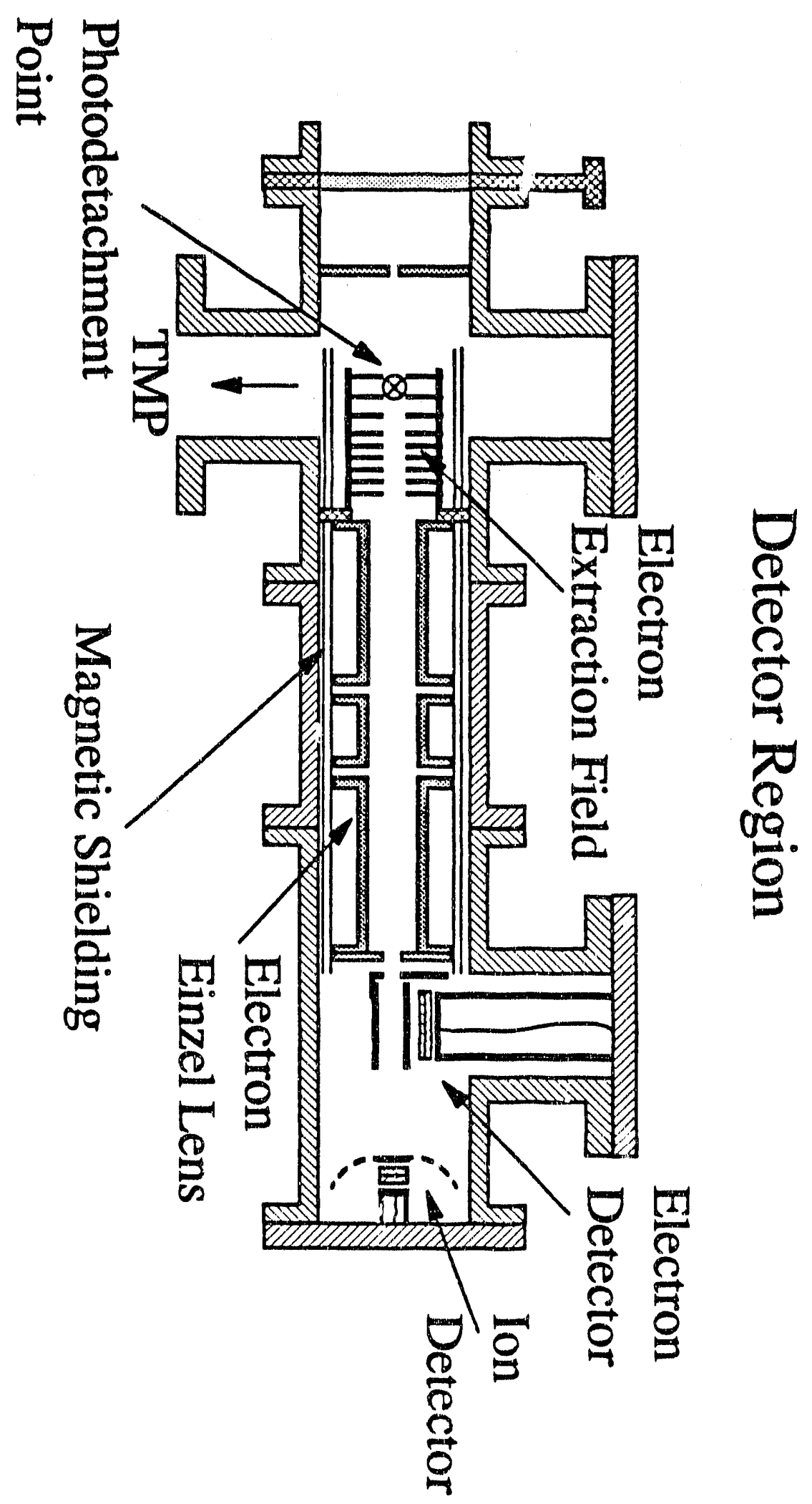

Figure 21 


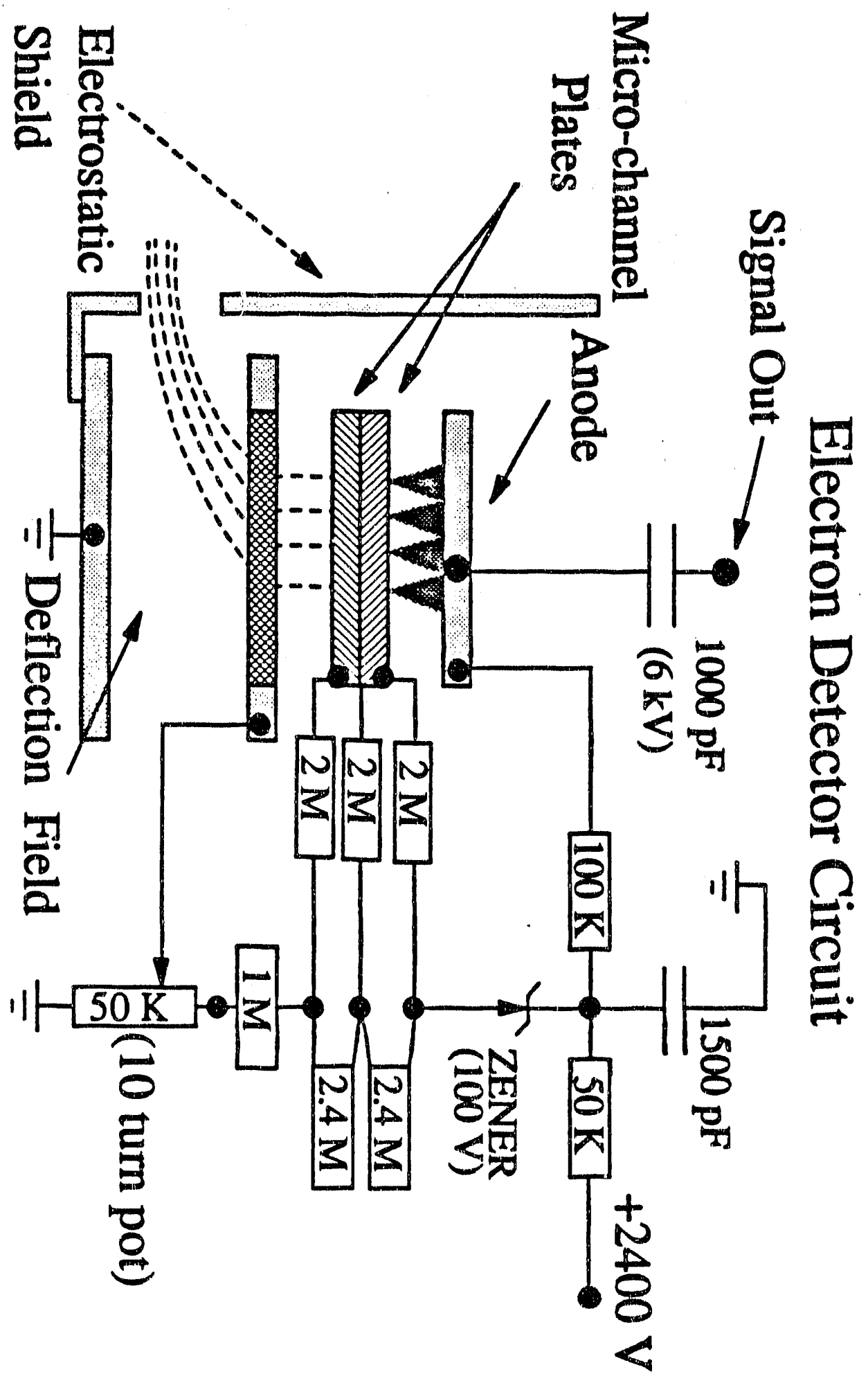

Figure 22 


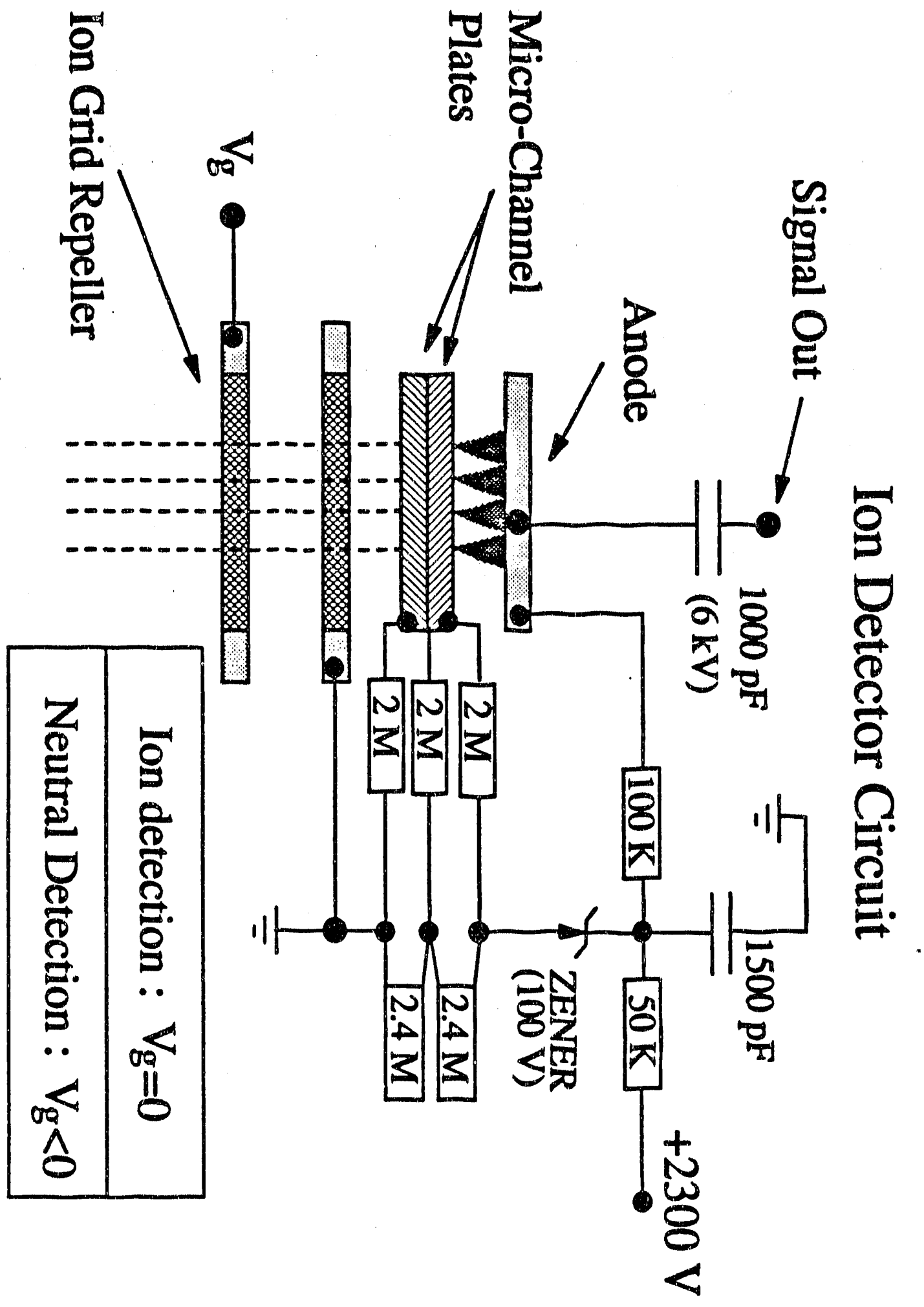

Figure 23 


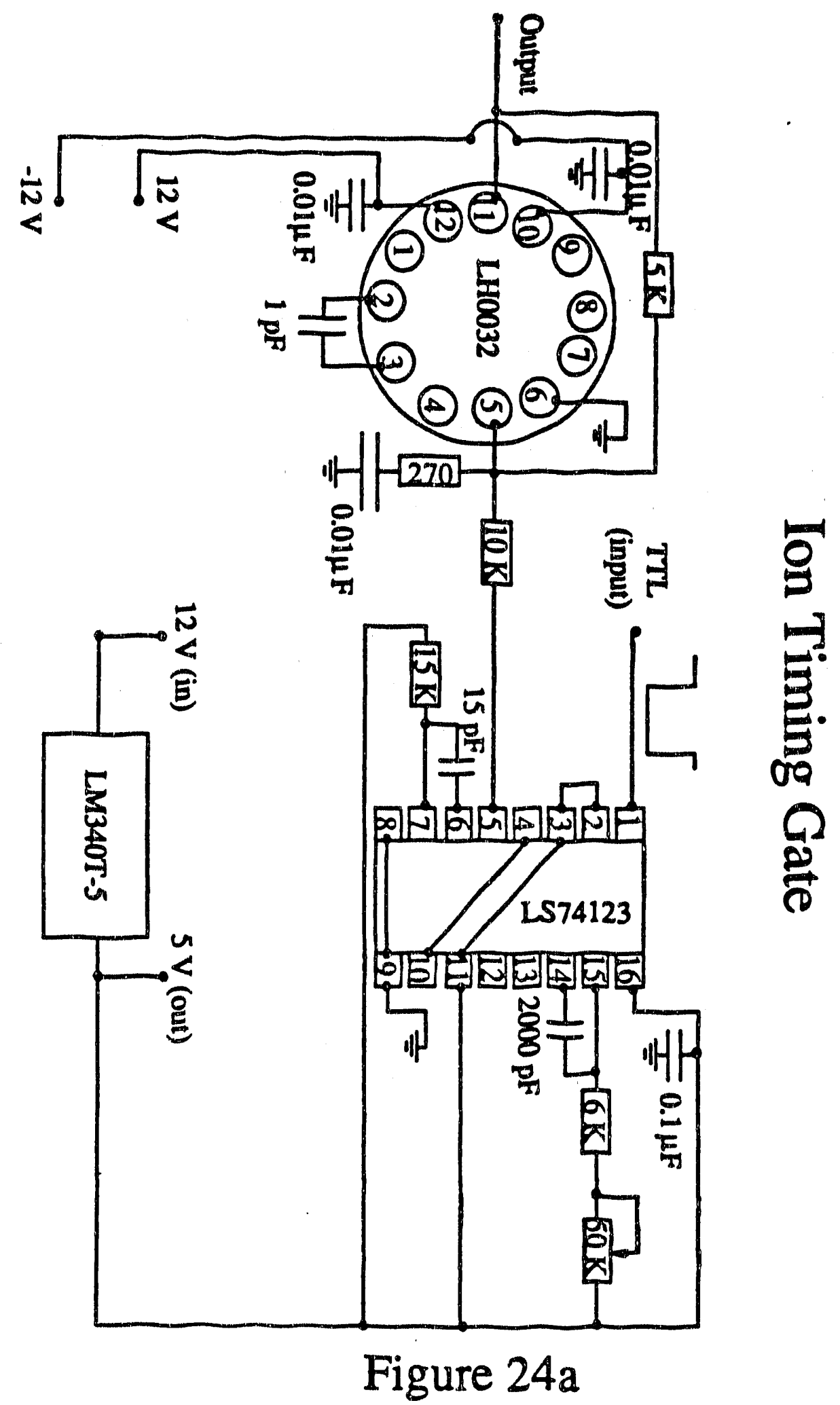




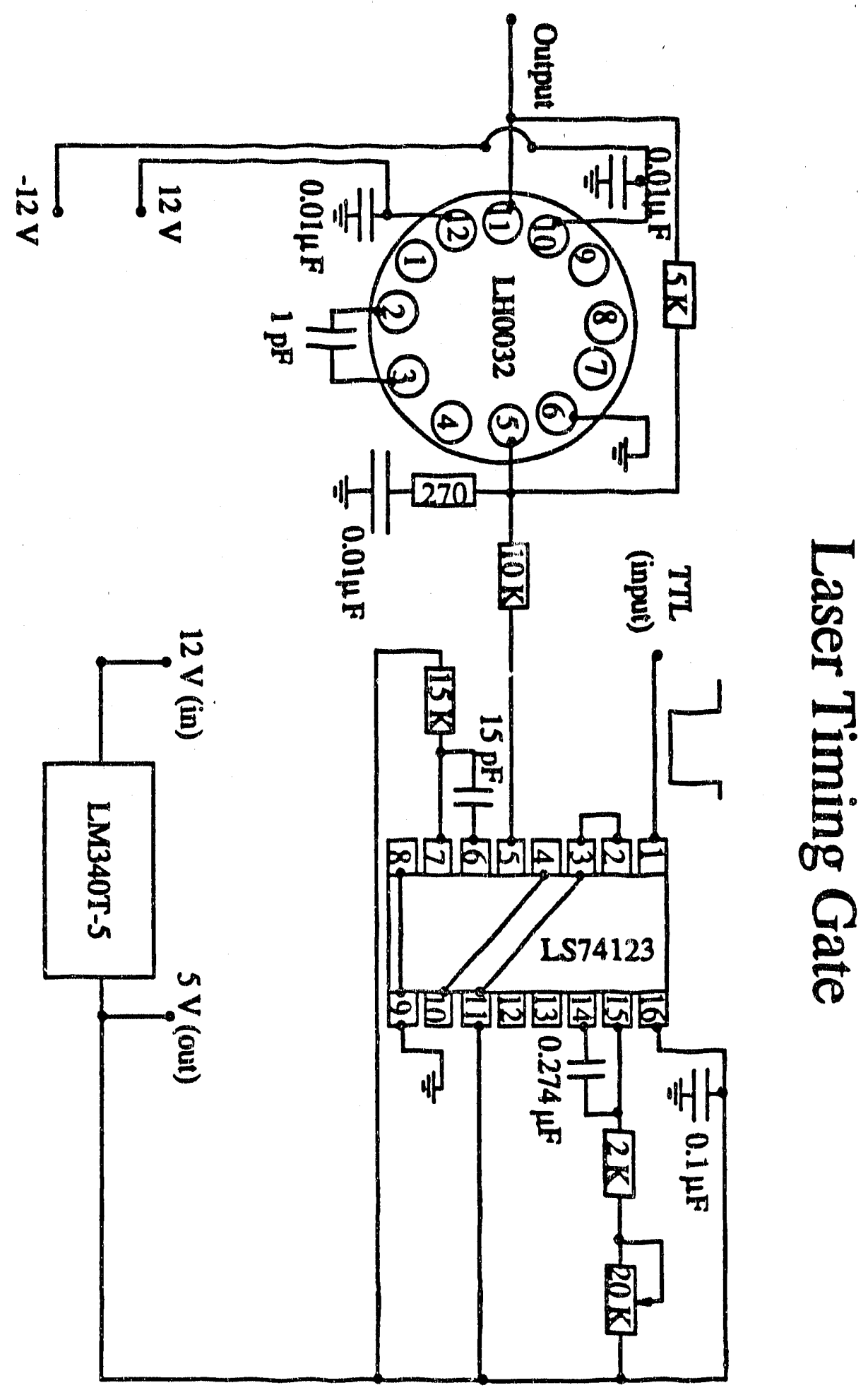

Figure 24b 

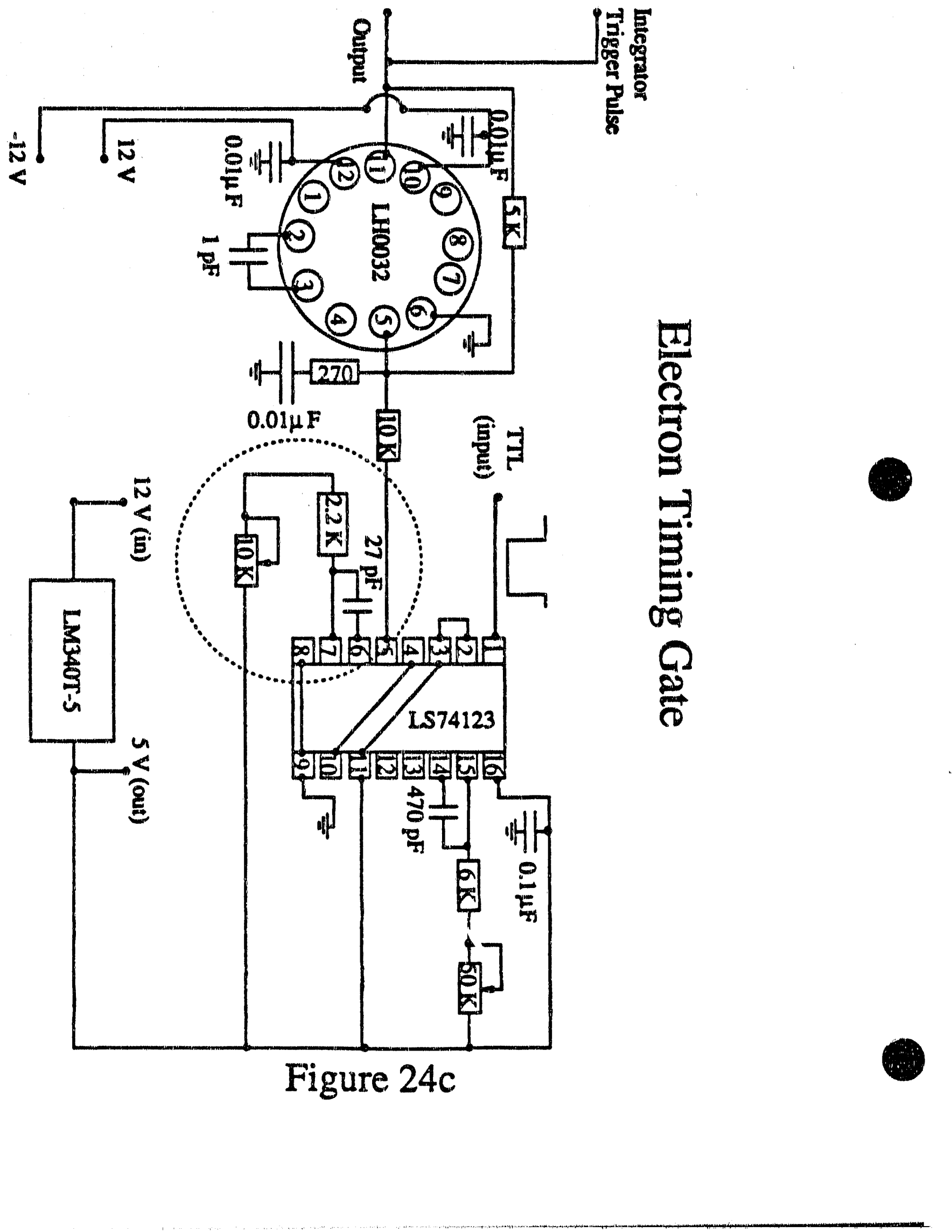

IS74123

言青

$$
\stackrel{3}{4}
$$




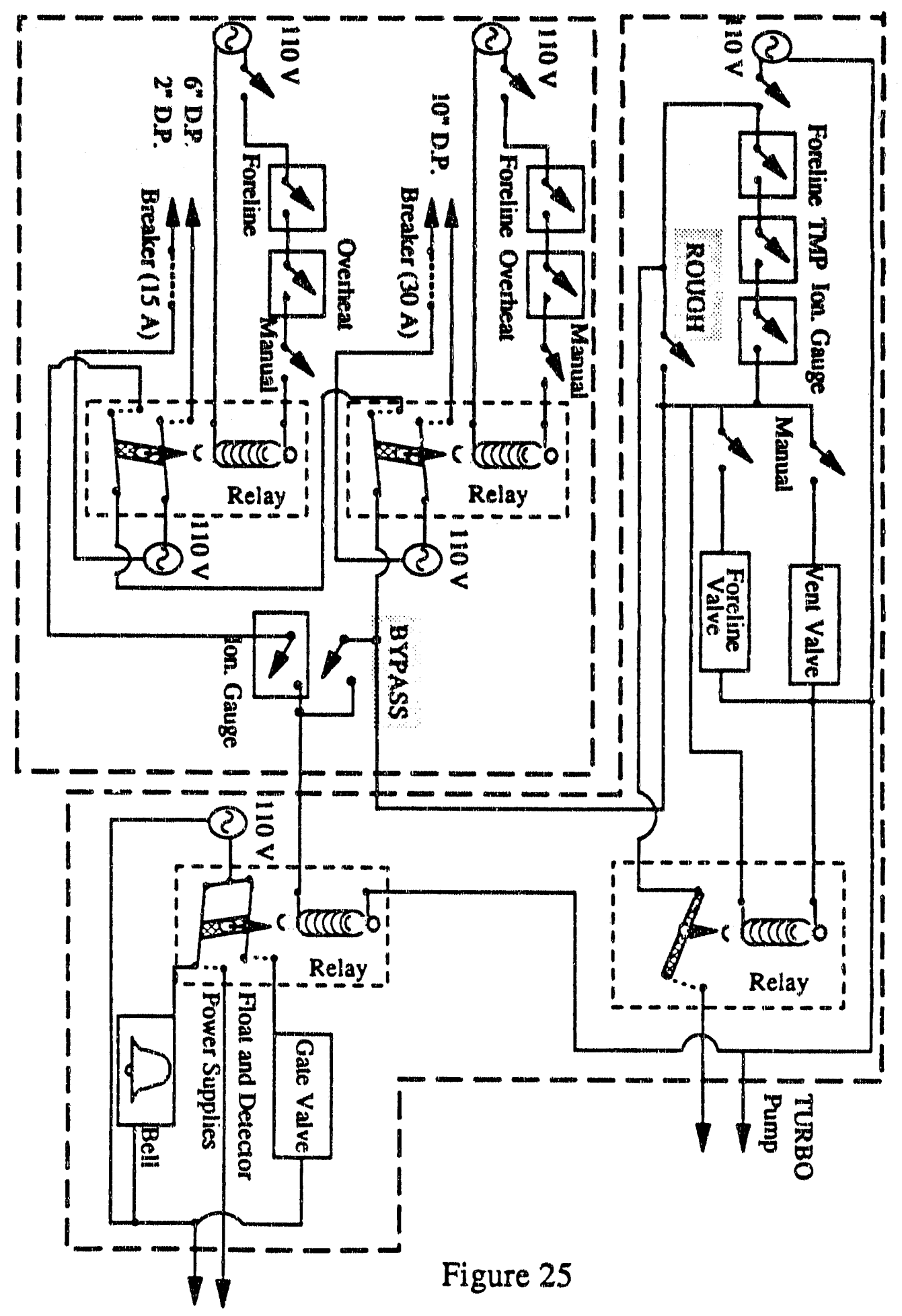




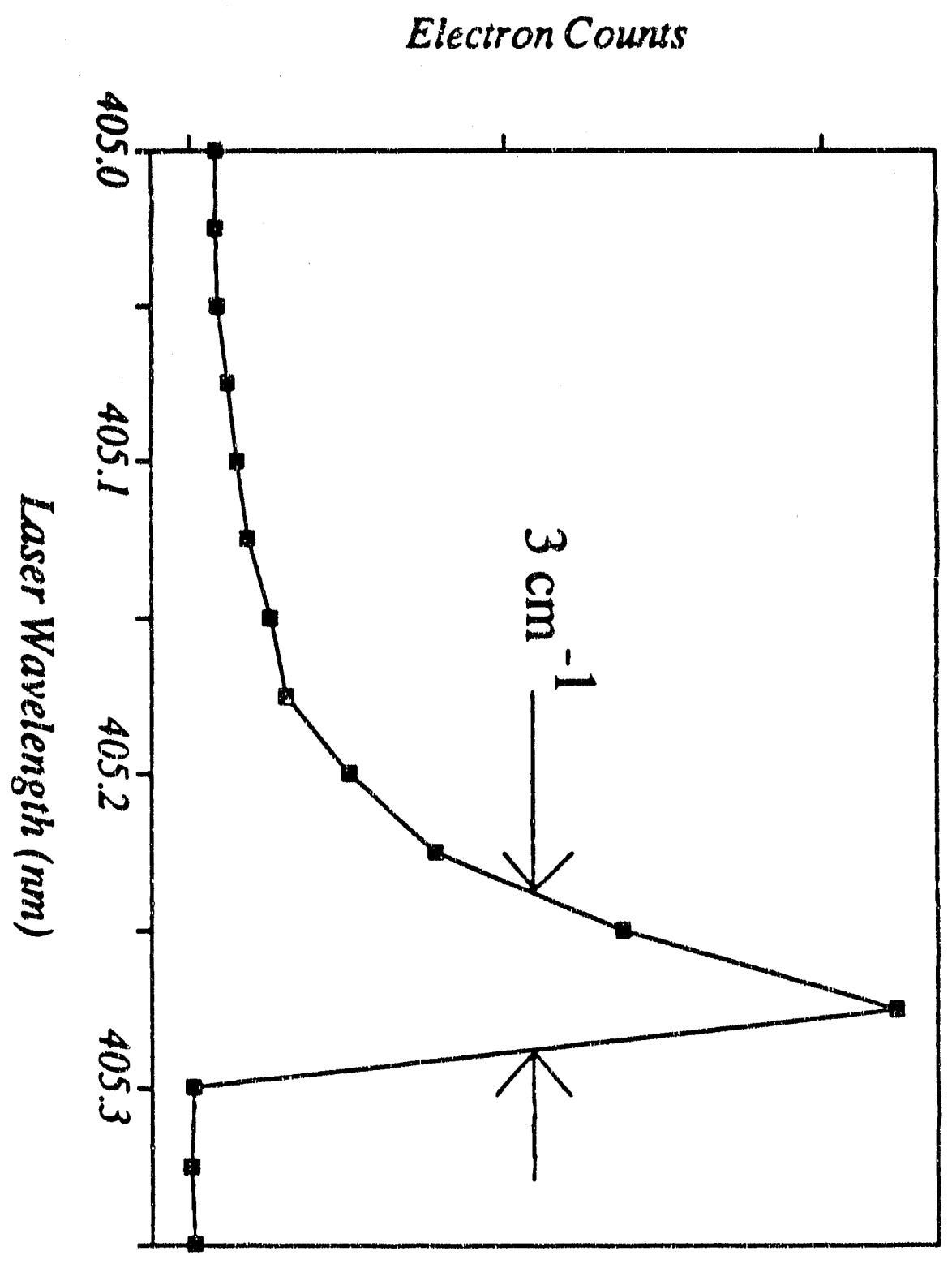

Figure 26 


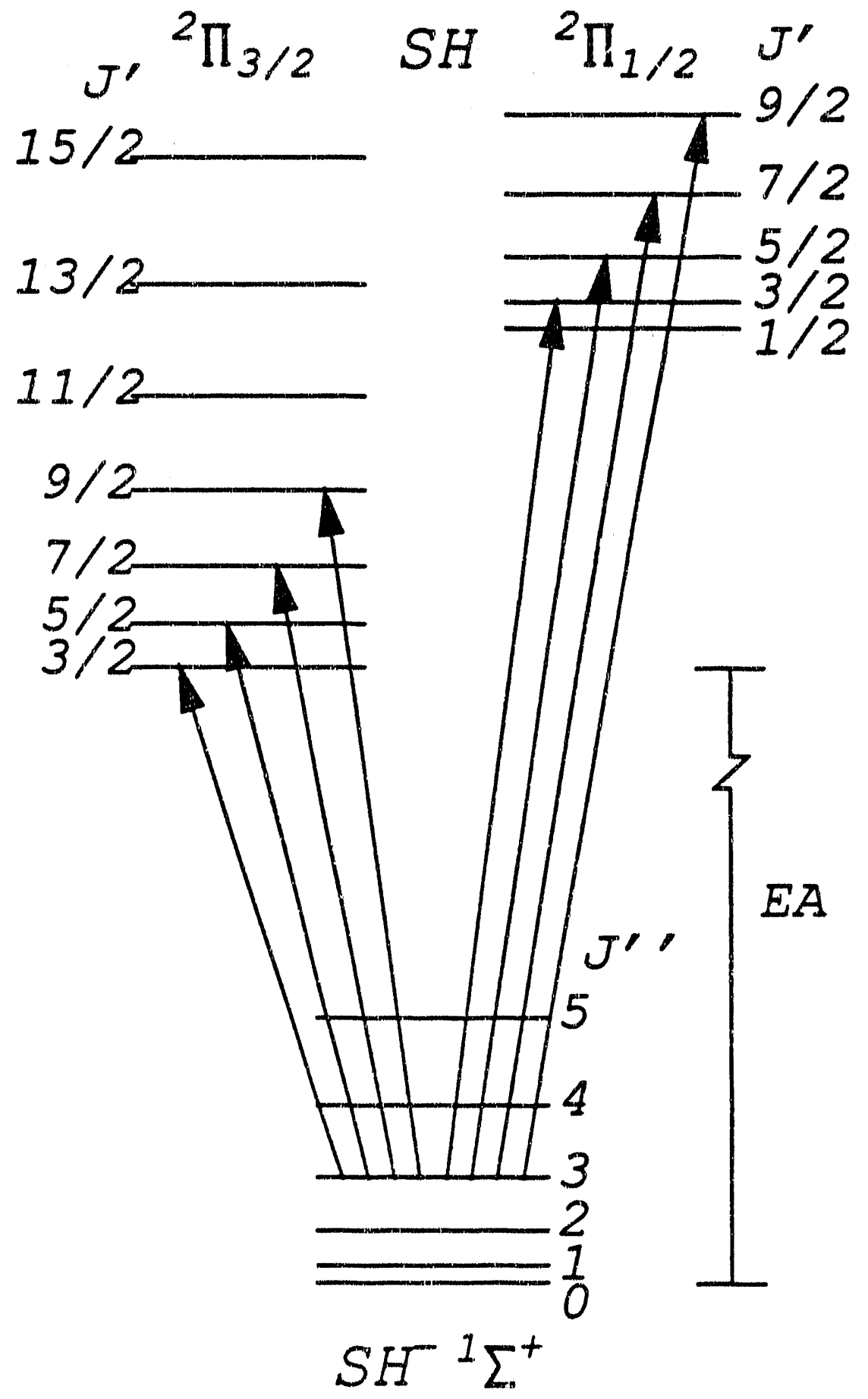

Figure 27 
Electron Counts

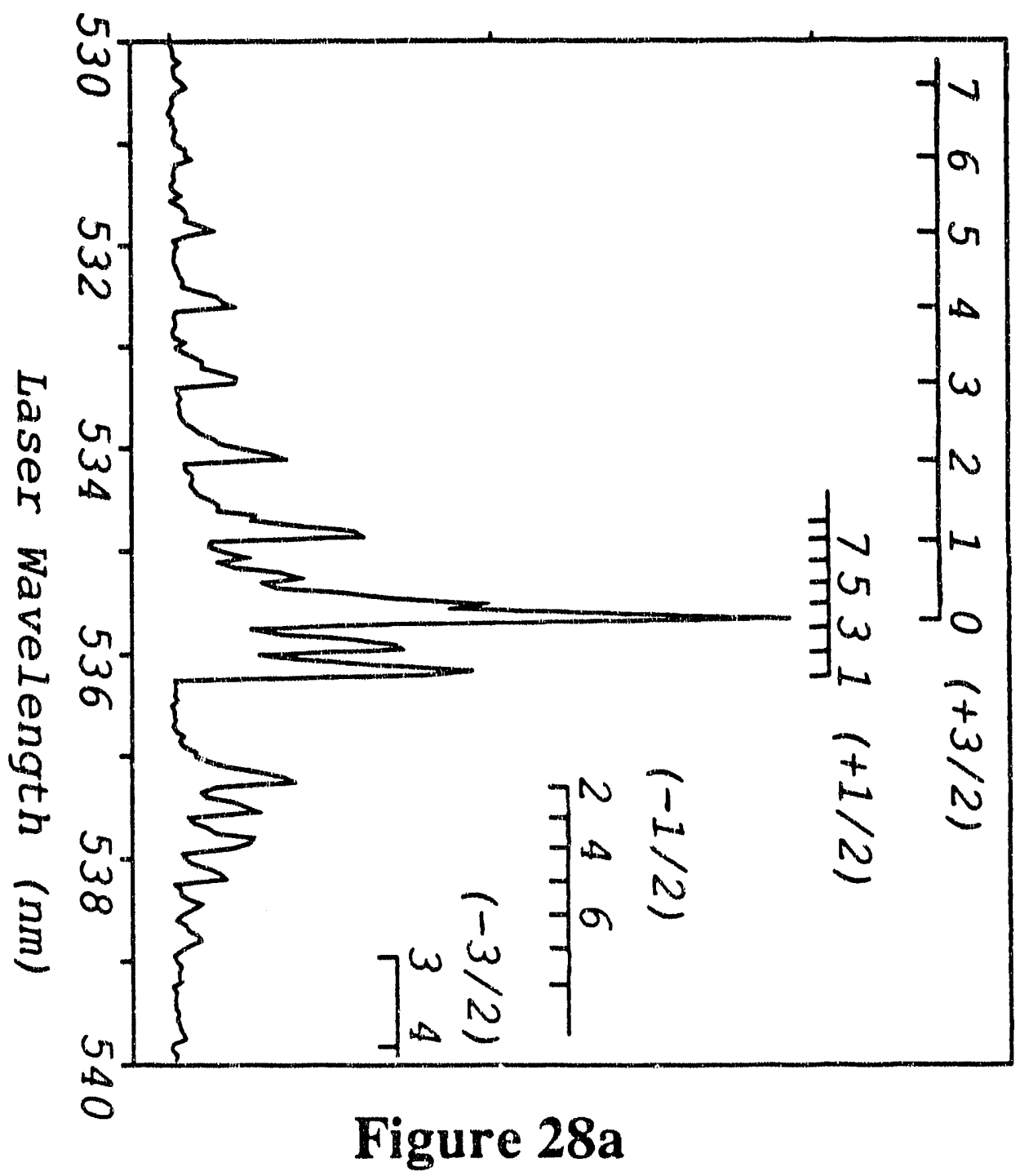




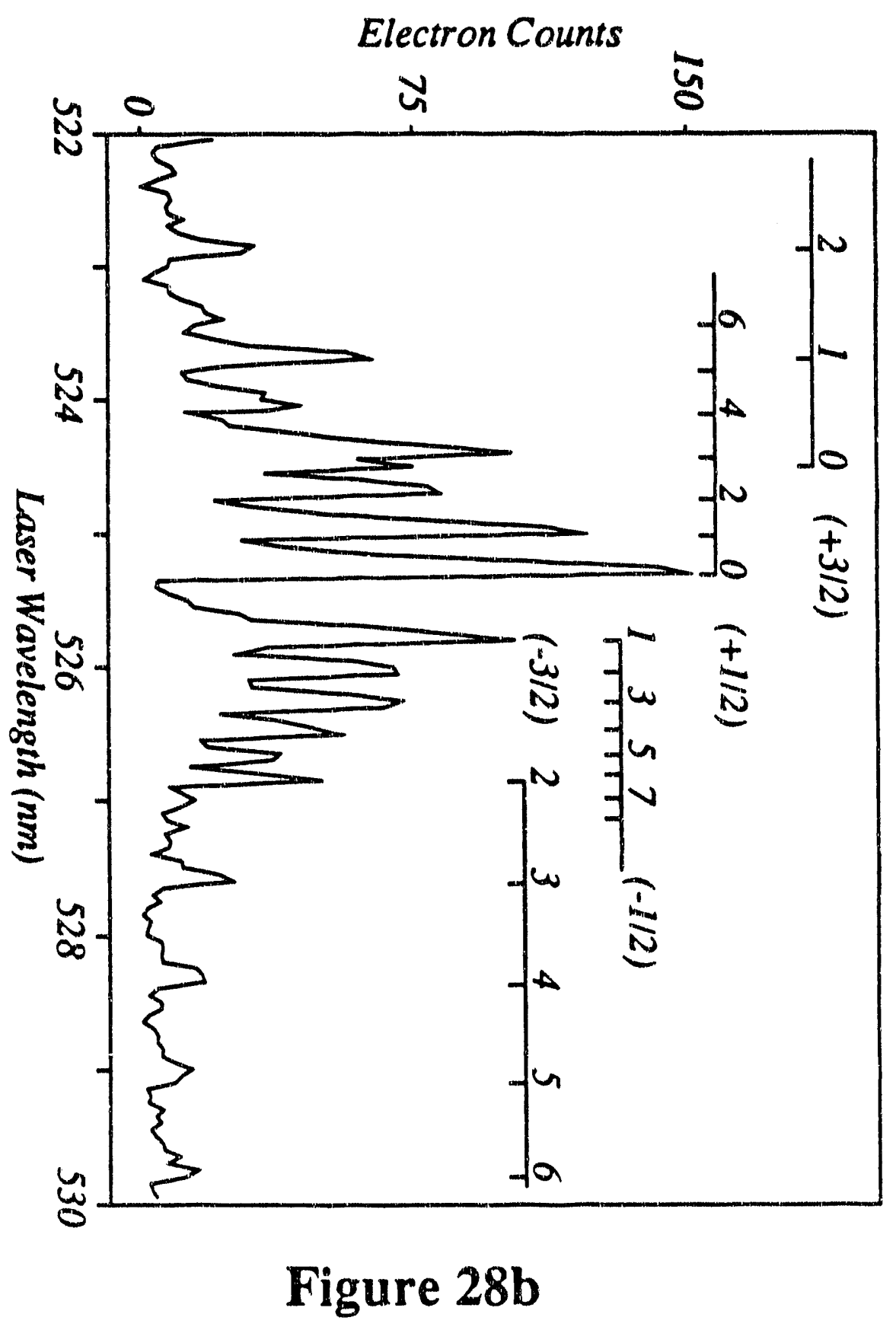




\section{References}

1. T.N. Kitsopoulos, I.M. Waller, J.G. Loeser, and D.M. Neumark, Chem. Phys. Lett. 159, 300 (1989).

2. J.R. Reitz, F.J. Milford, and R.W. Christy, "Foundations of Electromagnetic Theory" (Addison-Wesley Series in Physics, New York, 1980).

3. M.A. Johnson, M.L. Alexander, I. Hertel, and W.C. Lineberger, Chem. Phys. Lett. 12, 285 (1984).

4. SIMION PC/PS2 version 4.0, developed by Idaho National Engineering Laboratory.

5. H.S.W. Massey "Negative Ions" (Cambridge University Press, Cambridge, 1976).

6. B.M. Smimov "Negative Ions" ( McGraw-Hill Inc.,New York, 1982).

7. "Electron Molecule Interactions and Their Applications" edited by L.G. Christophorou (Academic Press Inc., New York, 1984).

8. J.F. O'Hanlon "A Users Guide to Vacuum Technology" (John Wiley and Sons, New York, 1980) pg. 135.

9. T.G. Dietz, M.A. Duncen, D.E. Powers, and R.E. Smalley, J. Chem. Phys. $\mathbf{7 4 , 6 5 1 1}$ (1981); V.E. Bondybey and J.H. English, J. Chem. Phys. 74, 6978 (1981).

10. J.F. Ready, "Effects of High Power Laser Radiation" (Academic, New York, 1971).

11. J. Bernholc and J.C. Phillips, J. Chem. Phys. 85, 3258 (1986).

12. E. Hecht and A. Zajac, "Optics" (Addison-Wesley Co., New York, 1979) pg. 246-250.

13. J.R. Heath, Y. Liu, S.C. O'Brien, Q.L. Zhang, R.F. Curl, F.K. Tittel, and R.E. Smalley, J. Chem. Phys. 83, 5520 (1985).

14. B. Kay, Ph.D. Thesis, University of Colorado, (1985). 
15. J.B. Anderson, R.P. Andres, and J.B. Fenn, "Molecular Beams", Edited by John Ross (John Wiley and Sons, New York, 1964).

16. M.B. Bakker, J. Phys. E 6, 785 (1973); 7364 (1974).

17. T.K. Fowler and W.M. Good, Nucl. Instrum. Meth. 7, 245 (1960).

18. W.C. Wiley and I.H. McLaren, Rev. Sci. Instr. 26, 1150 (1955).

19. F. Rosebury, "Electron Tube and Vacuum Techniques" (Addison-Wesley Co., 1964).

20. T.E.H. Walker, Chem. Phys. Lett 19, 493 (1973).

21. P.F. Bernath, T. Amano, and M. Wang, J. Mol. Spectr. 98, 20 (1983).

22. M. Gruebele, M. Polak, and R.J. Saykally, J. Chem. Phys. 86, 1698 (1987).

23. B. Steiner, J. Chem. Phys. 49, 5097 (1968).

24. B.K. Janousek and J.L. Brauman, Phys. Rev. A 23, 1673 (1981).

25. D.J. Larson, C.J. Edge, R.E. E.lmquist, N.B. Mansour, and R. Trainham, Physica Scripta T22, 183 (1988).

26. F. Breyer, P. Frey and H. Hotop, Z. Physik A 7, 300 (1981). 


\section{Chapter III}

Threshold photodetachment spectroscopy study of the transition state region

$$
\text { for the } \mathrm{I}+\mathrm{HI} \text { and } \mathrm{I}+\mathrm{DI} \text { reactions }
$$

\section{Introduction}

In recent years, we have developed a method for probing the transition state region of a neutral, bimolecular reaction via photodetachment of a stable negative ion which is similar in structure to the neutral transition state. ${ }^{1,2}$ In several cases, we have found that the photoelectron spectrum of the hydrogen-bonded anion $\mathrm{AHB}^{--}$exhibits resolved vibrational structure which can be assigned to an unstable [AHB] complex located near the transition state for the hydrogen transfer reaction $\mathrm{A}+\mathrm{HB} \rightarrow \mathrm{HA}+\mathrm{B}$. The results reported previously were obtained with a fixed-frequency negative ion photoelectron spectrometer. Here we report considerably higher resolution results for $\mathrm{HH}^{-}$, and $\mathrm{IDI}^{-}$ obtained on a recently constructed threshold photodetachment spectrometer. ${ }^{3}$ In this experiment, mass-selected ions are photodetached with a tunable pulsed laser, and only those photoelectrons produced with nearly zero kinetic energy are detected as a function of laser wavelength. The threshold photodetachment spectrum of $\mathrm{IHI}^{-}$shows considerably more structure than was observed in the lower resolution photoelectron spectrum. In particular, w observe narrow $\left(30 \mathrm{~cm}^{-1}\right)$ peaks due to long-lived states of the $[\mathrm{IHI}]$ complex which lie well above the $1+\mathrm{HI}(\mathrm{v}=0)$ asymptote.

\section{Experimental}

The high resolution threshold photodetachment spectrometer has been described in detail previously. ${ }^{3} \mathrm{IHI}^{-}$(IDI $)$anions are generated by expanding a $10 \%$ mixture of 
HI (DI) in either helium or argon through the 0.030 " diameter orifice of a pulsed molecular beam valve. Just outside the valve orifice, the molecular beam is crossed with a $1 \mathrm{keV}, 250 \mu \mathrm{A}$ electron beam. Negative ions are formed in the continuum flow region of the free jet expansion and internally cool as the expansion progresses; we have measured a rotational temperature of $75-100 \mathrm{~K}_{\text {for }} \mathrm{SH}^{-}$generated in this source (chapter II). The ions are accelerated coaxially to $500 \mathrm{eV}$ and mass-selected with a beammodulated time-of-flight mass spectrometer. ${ }^{4}$

The mass-selected $\mathrm{IHI}^{-}(\mathrm{IDI}-7)$ anions are photodetached using an excimer-pumped frequency-doubled tunable dye laser operating at $50 \mathrm{~Hz}$. Rhodamine 590,610 and 640 laser dyes were used and doubled with either KDP or BBO resulting in 3-5 mJ/pulse. The wavelength scale was calibrated using the optogalvanic effect in a hollow cathode neon discharge.

Our: photoelectron detection scheme is based on the design of Schlag and coworkers ${ }^{5}$ for threshold photoionization of neutrals. A delay of $180 \mathrm{~ns}$ subsequent to photodetachment allows the more energetic electrons and the threshold electrons to separate. A weak field is applied along the ion beam axis to extract the photoelectrons. Electrons produced with nearly zero kinetic energy are selectively detected using a combination of spatial and temporal filtering. The electron signal is normalized to the laser power and the total ion signal.

\section{Results and Discussion}

\section{a. Photodetachment of $\mathrm{IHI}^{-}$}

The new results should be compared to the $\mathrm{IHI}^{-}$photoelectron spectrum shown 
in Figure 1 which was obtained previously on our fixed-frequency spectrometer. ${ }^{2}$ In this spectrum, the ions were photodetached using the 4th harmonic of a Nd:YAG laser (266 $\mathrm{nm}$ or $4.66 \mathrm{eV}$ ). The resolution of the instrument is $8 \mathrm{meV}$ for $0.65 \mathrm{eV}$ electrons and degrades as $E^{3 / 2}$ at higher electron kinetic energy. Three peaks of varying widths are observed; these are assigned to transitions to the $v_{3}{ }^{\prime}=0,2$, and 4 antisymmetric stretch levels of the $[\mathrm{IH}]$ complex. All three peaks appear to lie above the $\mathrm{I}+\mathrm{HI}(\mathrm{v}=0)$ asymptote (which occurs at an electron kinetic energy of $0.86 \pm 0.13 \mathrm{eV}^{6}$ ) and therefore correspond to levels of the [IHI] complex with sufficient energy to dissociate. The spacing between the peaks is substantially less than the vibrational frequency in diatomic $\mathrm{HI}$, indicating that photodetachment of $\mathrm{IHI}^{-}$accesses the transition state region of the I + HI potential energy surface where the $\mathrm{H}$ atom is interacting strongly with the two I atoms.

A vital question in the interpretation of this spectrum is whether the peak widths in Figure 1 are determined solely by the lifetime of the various [IHI] $v_{3}$ levels or are instead envelopes for unresolved fine structure. Using a model potential energy surface for the $\mathrm{I}+\mathrm{HI}$ reaction, approximate simulations of the $\mathrm{IHI}^{-}$photoelectron spectrum by ourselves $^{2}$ and more sophisticated simulations by Schatz ${ }^{7,8,9}$ and Bowman ${ }^{10}$ have suggested that higher resolution studies of the $\mathrm{v}_{3}{ }^{\prime}=2$ and 4 peaks in Figure 1 might reveal progressions of closely spaced metastable symmetric stretch levels of the [IHI] complex. These levels are of considerable interest since they are responsible for the sharp resonance structure seen in scattering calculations on heavy + light-heavy reactions. ${ }^{11}$

The threshold photodetachment spectra of the three peaks in Figure 1 are shown 
in Figures 2-4. The horizontal axis at the bottom of each plot shows the photodetachment wavelength $\lambda$ in nanometers. For ease of comparison with Figure 1, the top axis shows the corresponding electron kinetic energy (eKE), in electron volts, that would result in a photoelectron spectrum at $266 \mathrm{~nm}$; the two axes are related by eKE $=1240(1 / 266-1 / \lambda)$.

The threshold photodetachment spectrum of the $v_{3}{ }^{\prime}=2$ peak (Figure 3) reveals three partially resolved peaks spaced by approximately $100 \mathrm{~cm}^{-1}(12.4 \mathrm{meV})$. This frequency is in the range expected for symmetric stretch levels of the [IHI] complex. In fact, simulations of transitions to the $v_{3}{ }^{\prime}=2$ level of [IHI] are quite similar in appearance to the experimental result; they also show a symmetric stretch progression of three peaks decreasing in intensity towards lower wavelength. ${ }^{7,10}$ The experimental peak widths of approximately $100 \mathrm{~cm}^{-1}(12.4 \mathrm{meV})$ are considerably broader than the predicted resonance widths of $16 \mathrm{~cm}^{-1}(2 \mathrm{meV}){ }^{7}$ Nonetheless, the correspondence between the experimental and simulated spectra strongly suggests we are seeing a progression in quasi-bound [IHI] symmetric stretch states.

Further evidence for this comes from Figure 4 which shows the threshold photodetachment spectrum to $\mathrm{v}_{3}{ }^{\prime}=4$ levels of [IHI]. Four well-resolved peaks are evident in this spectrum. The peaks are substantially narrower than those in Figure 3; peaks A and a are $30 \mathrm{~cm}^{-1}$ wide, and peak B is $45 \mathrm{~cm}^{-1}$ wide. Peaks A and a are separated by 134 $\mathrm{cm}^{-1}$, peaks $\mathrm{a}$ and $\mathrm{b}$ by $130 \mathrm{~cm}^{-1}$, and peaks $\mathrm{A}$ and $\mathrm{B}$ by $93 \mathrm{~cm}^{-1}$. This pattern suggests that peaks $\mathrm{a}, \mathrm{b}$ and $\mathrm{A}$ form one progression, and peaks $\mathrm{A}$ abd $\mathrm{B}$ form a second.

The intensities of peaks $a$ and $b$ are sensitive to the ion source operating conditions. Matrix isolation studies on $\mathrm{IHI}^{-}$have yielded a value of $130 \mathrm{~cm}^{-1}$ and 126 
$\mathrm{cm}^{-1}$ for the $0 \rightarrow 1$ and $1 \rightarrow 2$ symmetric stretch transitions in the ion respectively. ${ }^{12}$ We therefore assign peaks $a$ and $b$ to hot band transitions originating from the $v_{1}{ }^{\prime \prime}=1$ and $v_{1}{ }^{\prime}=2$ symmetric stretch levels of the ion and terminating in the same level of the neutral as peak $\mathrm{A}$. The a/A intensity ratio yields a vibrational temperature of $100 \mathrm{~K}$ for the $\mathrm{IHI}^{-}$ions.

Peaks $A$ and $B$ belong to a second progression and are assigned to transitions between the $v_{1} "=0$ level of the ion and two different symmetric stretch levels of the [IHI] complex. This assignment is consistent with the noticeably different peak widths which imply that the transitions are to two distinct levels of the complex with different lifetimes. The widths yield lower bounds of 180 and 120 fs for the upper state lifetimes of $A$ and $B$, respectively. Since the symmetric stretch vibration of $[\mathrm{IHI}]$ is strongly coupled to the dissociation coordinate of the complex, it seems reasonable that the higher lying symmetric stretch level of the complex (peak B) should have a shoner lifetime. Similar trends have been observed in collinear scattering calculations on the $\mathrm{Cl}+\mathrm{HCl}$ and $\mathrm{F}+\mathrm{DBr}$ reactions. ${ }^{13}$ Note that a substantial portion of the width of peak $\mathrm{A}$ is due to the experimental resolution, so the upper state lifetime for this transition may be considerably longer.

The symmetric stretch levels of the $[\mathrm{IHI}]$ complex with $\mathrm{v}_{3}{ }^{\prime}=4$ lie $0.49 \mathrm{eV}$ above the $\mathrm{I}+\mathrm{HI}(\mathrm{v}=0)$ asymptote compared to $0.30 \mathrm{eV}$ for the levels with $\mathrm{v}_{3}{ }^{\prime}=2$. The peak. widths in Figures 3 and 4 , however, indicate that the lifetimes of the $v_{3}{ }^{\prime}=4$ levels are considerably longer than the $v_{3}^{\prime}=2$ levels. Although this result may seem surprising, the $V_{3}$ antisymmetric stretch vibration is expected to be poorly coupled to the dissociation 
coordinate of the [IHI] complex. In fact, wn the I + HI surface used in the simulations, the vibrationally adiabatic potential for the $v_{3}=4$ level of [IH] has a deeper well than the adiabatic potential for the $v_{3}=2$ level, suggesting that states of the complex with $v_{3}$ $=4$ are more effectively 'trapped' in the transition s‘ate region. In his three-dimensional simulations of the $\mathrm{IDI}^{-}$photoelectron spectrum, Schatz ${ }^{7,9,14}$ found that transitions to [IDI] symmetric stetch levels with $v_{3}{ }^{\prime}=4$ were indeed narrower than those with $v_{3}{ }^{\prime}=$ 2.

The threshold photodetachment spectrum for the transition to $v_{3}{ }^{\prime}=0$ is shown in Figure 4. It consists of a progression of broad peaks spaced by an interval that increases from $160 \mathrm{~cm}^{-3}$ to $200 \mathrm{~cm}^{-1}$ towards lower laser wavelength. This is quite different from the $100 \mathrm{~cm}^{-1}$ interval seen in the other spectra. In contrast to the $v_{3}^{\prime}=2$ and 4 states of [IHI], photodetachment to $v_{3}{ }^{\prime}=0$ is expected to primarily access direct scattering $I+H I$ wavefunctions rather than quasi-bound resonances. ${ }^{7,10}$ In light of this, the observation of any structure in the photodetachment spectrum is interesting. A possible explanation suggested by Schatz's $\mathrm{IHI}^{-}$simulation, arises from the correspondence between the peak positions in Figure 4 and the energetic thresholds for the asymptotic levels $I+H I(v=0$, $j=11-16)$. The threshold for these asymptotic states line up reasonably well with the peaks in the photodetachment spectrum as indicated in Figure 2. His assumes that the threshold for the $\mathrm{I}+\mathrm{HI}(\mathrm{v}=0, \mathrm{j}=0)$ is $3.79 \mathrm{eV}(327.14 \mathrm{~nm})$, well within the uncertainty in the experimental value of $3.80 \pm .13 \mathrm{eV} .^{15}$ Our results therefore indicate that photodetachment to the $v_{3}{ }^{\prime}=0$ level of [IHI] is enhanced near the thresholds for formation of $\mathrm{HI}(\mathrm{v}=0)$ in high rotational states. 
In his three-dimensional simulation of the $\mathrm{IHI}^{-}$photoelectron spectrum, Schatz ${ }^{7,9}$ also observed peaks in the transitions to $v_{3}{ }^{\prime}=0$ levels of the complex which correspond to asymptotic I + HI rotational energy levels. However, his results show significant intensity down to the $I+H I(j=0)$ level. The apparent drop in signal for $j<11$ may indicate that on the real $\mathrm{I}+\mathrm{HI}$ potential energy surface, the scattering wavefunctions wiich asymptotically correlate to $I+H I(v=0, j<11)$ do not have much amplitude in the region of the surface probed by photodetachment. Classical trajectory and quantum scattering calculations on model $\mathrm{Cl}+\mathrm{HCl}$ potential energy surfaces predict that rotational excitation of $\mathrm{HCl}(\mathrm{v}=0)$ dramatically increases its reactivity. ${ }^{16}$ A quasiclassical trajectory study of the $\mathrm{Br}+\mathrm{HBr}$ system also showed that the reaction rate was greatly enhanced for $\operatorname{HBr}(v=0, j>10) .{ }^{17}$ Our results are consistent with this type of effect occurring in I $+\mathrm{HI}$; that is, if relatively few $I+H I(v=0, j<11)$ collisions lead to reaction, one might expect that the corresponding scattering wavefunctions will not penetrate into the transition stare region.

\section{b. Photodetachment of IDI $^{-}$}

The photoelectron spectrum of IDI- obtained at $2.66 \mathrm{eV}$ is shown in Figure $5^{2}$. The appearance of the spectrum is similar to that of $\mathrm{IHI}^{-}$; the origin of the spectrum does not appear to have shifted while the peak splittings are now smaller. This supports our assignment of this spectral features to transitions involving the antisymmetric stretch which corresponds to the fast $H(D)$ motion. Once again the vital question we would like to answer is whether or not the spectral peak widths are solely determined by lifetime or is their underlying unresolved structure (resonances), analogous to those observed in the 
$\mathrm{IHI}^{-}$photodetachment spectra.

The threshold spectrum for the transition to the $v=2$ and 4 antisymmetric stretch level of [IDI] is shown in fig. 7 and 8 . The $v=2$ spectrum consists of two partially resolved peaks separated by $270 \mathrm{~cm}^{-1}$; however the position of the higher energy peak corresponds very closely to the IHI $(v=2)$ peak of fig. 2 . Hence we believe that this additional structure in our $\mathrm{IDI}^{-}$spectrum is due to $\mathrm{IHI}^{-}$contamination in our precursor anions, which our mass spectrometer could not resolve very efficiently. The width (FWHM) of the peak that does correspond to the [IDI] $\mathrm{v}=2$ level is about $160 \mathrm{~cm}^{-1}$.

The threshold spectrum for the transition to the $v_{3}=4$ level of [IDI] also consists of two peaks. Psak a is located about $140 \mathrm{~cm}^{-1}$ to the red of peak $A$, and is sensitive to the source conditions. We assign this to a hot band transition from the $v^{\prime \prime}=1$ symmetric stretch level of the anion to the ground vibrational state of the neutral and the frequency compares well to the $124 \mathrm{~cm}^{-1}$ value for the $\mathrm{IDI}^{-}$symmetric stretch observed in a matrix. ${ }^{12}$ Peak $A$ is $60 \mathrm{~cm}^{-1}$ wide, is considerably narrower than the peak corresponding to the transition to the $v_{3}{ }^{\prime}=2$ [IDI] level. We observe that the peak widths for the spectra of figures 7 and 8 are comparable to the $100 \mathrm{~cm}^{-1}$ and $25 \mathrm{~cm}^{-1}$ widths of the peaks in the [IH] $v=2$ and 4 spectra of fig. 3 and 4 . This strongly suggests that we are observing transitions to symmetric stretch levels bound by the $v_{3}{ }^{\prime}=2$ and 4 adiabatic potential of [IDI] respectively. However, unlike the [IHI] spectrum, we do not see a progression in this mode in either level.

One possibility is that the well depth of the adiabatic potentials are too shallow and thus support only one level. Alternatively, lets assume that the symmetric stretch 
frequency for $[\mathrm{IDI}]$ is similar to the $[\mathrm{IHI}]$ i.e. about $100 \mathrm{~cm}^{-1}$. Then for the transition to the $v_{3}{ }^{\prime}=2$ level of $[\mathrm{IDI}]$, it could be very difficult to resolve two peaks separated by this amount if their widths are $\geq 160 \mathrm{~cm}^{-1}$. On the other hand, the absence of a symmetric stretch progression in the transition to the $v_{3}{ }^{\prime}=4$ level of [IDI], would imply that the Franck-Condon overlap to higher symmetric stretch levels is very small and are consequently not observed. In addition, the fact that peak $\mathrm{A}$ of fig. 8 is only 2.5 times broader than the corresponding transition in the $\mathrm{IHI}^{-}$threshold spectrum (peak $\mathrm{A}$, fig. 4), leads us to assign this peak to a resonance.

In the $\mathrm{IDI}^{-}$spectra, as in the $\mathrm{IHI}^{-}$spectra, the peak widths get narrower as the antisymmetric stretch level increases. Hence the absence of structure due to quasi-bound resonances in the transition to the $v_{3}{ }^{\prime}=0$ level of [IDI] (fig. 6) is not disappointing but rather reassuring. However in his three-dimensional simulation of the $\mathrm{IDI}^{-}$photoelectron spectrum, Schatz ${ }^{7,14.9}$ does observe transitions to the $v_{3}^{\prime}=0$ level of [IDI] which correspond to asymptotic I + DI rotational levels, similar to those observed in the $\mathrm{IHI}^{-}$ $v=0$ threshold spectrum (fig. 2). Unfortunately our $\mathrm{IDI}^{-}, \mathrm{v}=0$ spectrum shows no evidence of such direct scattering resonances. Part of this might have to do with the magnitude of the rotational constant of DI is approximately half that of $\mathrm{HI}$; this implies that the average separation between the high rotational levels $(j \geq 7)$ will be on the order of 80 to $100 \mathrm{~cm}^{-1}$. Consequently, if the life time of these resonances are similar to those in [IH], they would correspond to peak widths of $100 \mathrm{~cm}^{-1}$, hence resolving individual transitions to these hindered rotor states will be extremely difficult.

\section{Conclusion}


The high resolution threshold photodetachment spectra of $\mathrm{IHI}^{-}$reveals progressions in closely-spaced symmetric stretch levels of the [IHI] complex which lie well above I $+\mathrm{HI}(\mathrm{v}=0)$. The narrow peaks observed in the spectra demonstrate the existence of the relatively long-lived metastable levels of the [IH] complex which lead to resonances in the I+ HI reaction. We also observe structure associated with I + $\mathrm{HI}(\mathrm{v}=0, \mathrm{j})$ asymptotic levels for $\mathrm{j}>11$ which may incicate enhanced reactivity for rotationally excited states of $\mathrm{HI}(\mathrm{v}=0)$. The spectra presented here represent a significant step towards the spectroscopic characterization of the transition state region in a chemical reaction. The threshold photodetachment spectra of $\mathrm{IDI}^{-}$also showed evidence of resonances due to quasi-bound symmetric stretch levels, but the overall assignment of the observed spectrum is less certain. 


\section{Figure Captions}

1. The fixed-frequency (at $4.66 \mathrm{eV}, 266 \mathrm{~nm}$ ) photoelectron spectrum of $\mathrm{IHI}^{-}$reveals a progression in the antisymmetric stretch mode of the [IHI].

2. The threshold photodetachment spectrum of $\mathrm{IHI}^{-}$for the transition to the $\mathrm{v}_{3}{ }^{\prime}=0$ antisymmetric stretch level of [IHI].

3. Threshold photodetachment spectrum of $\mathrm{IHI}^{-}$for the transition to the $\mathrm{v}_{3}{ }^{\prime}=2$ antisymmetric stretch level of the [IHI].

4. Threshold photodetachment spectrum of $\mathrm{IHI}^{-}$for the transition to the $\mathrm{v}_{3}{ }^{\prime}=4$ antisymmetric stretch level of the [IHI].

5. Photoelectron spectrum of $\mathrm{IDI}^{-}$obtained at $266 \mathrm{~nm}(4.66 \mathrm{eV})$ revealing a progression in the antisymmetric stretch mode of the [IDI] complex.

6. Threshold photodetachment spectrum of $\mathrm{IDI}^{-}$for the transition to the $\mathrm{v}_{3}{ }^{\prime}=0$ antisymmetric stretch level of [IDI].

7. Threshold photodetachment spectrum of $\mathrm{IDI}^{-}$for the transition to the $\mathrm{v}_{3}^{\prime}=2$ antisymmetric stretch level of [IDI].

8. Threshold photodetachment spectrum of $\mathrm{IDI}^{-}$for the transition to the $\mathrm{v}_{3}{ }^{\prime}=4$ antisymmetric stretch level of [IDI]. 

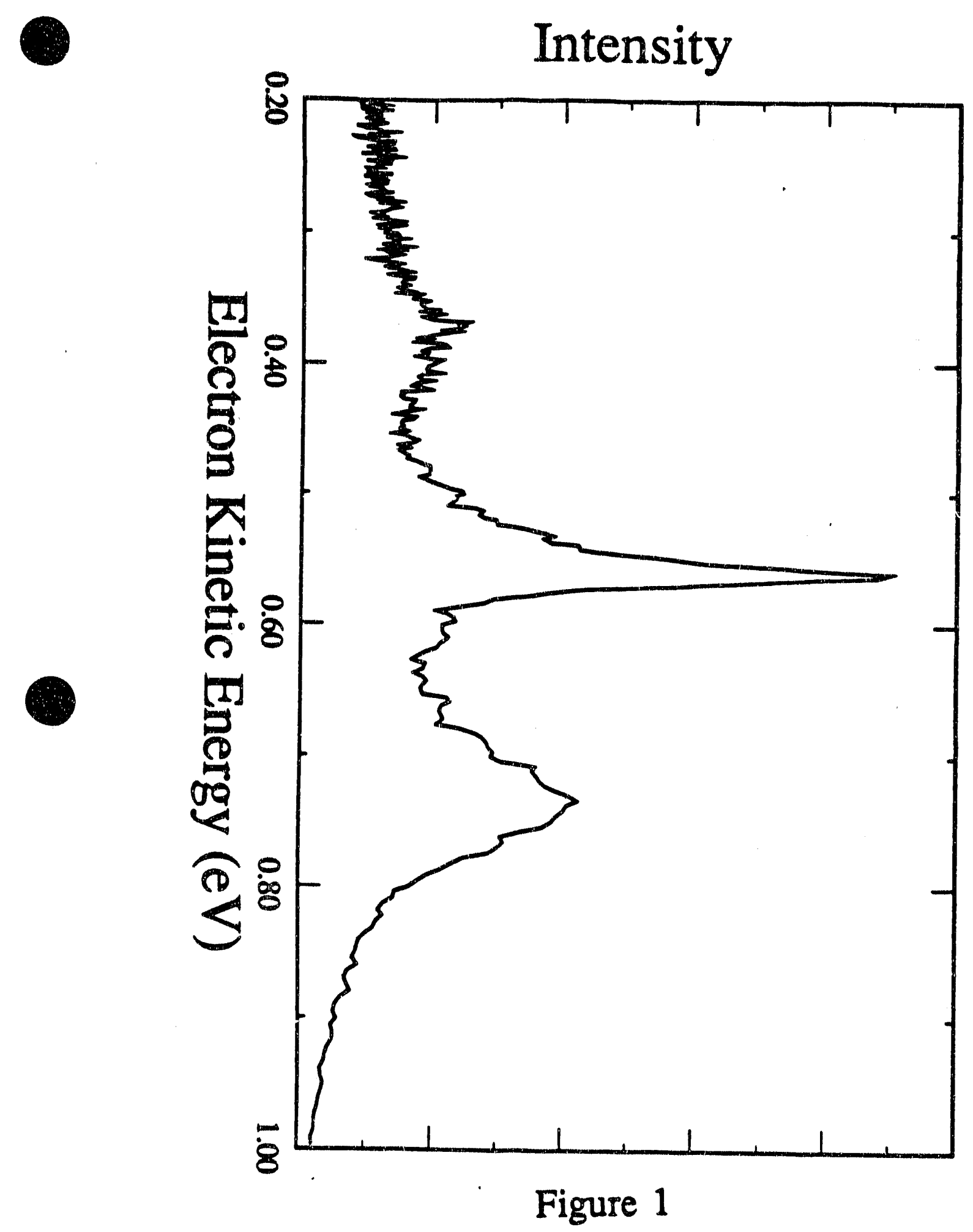
106

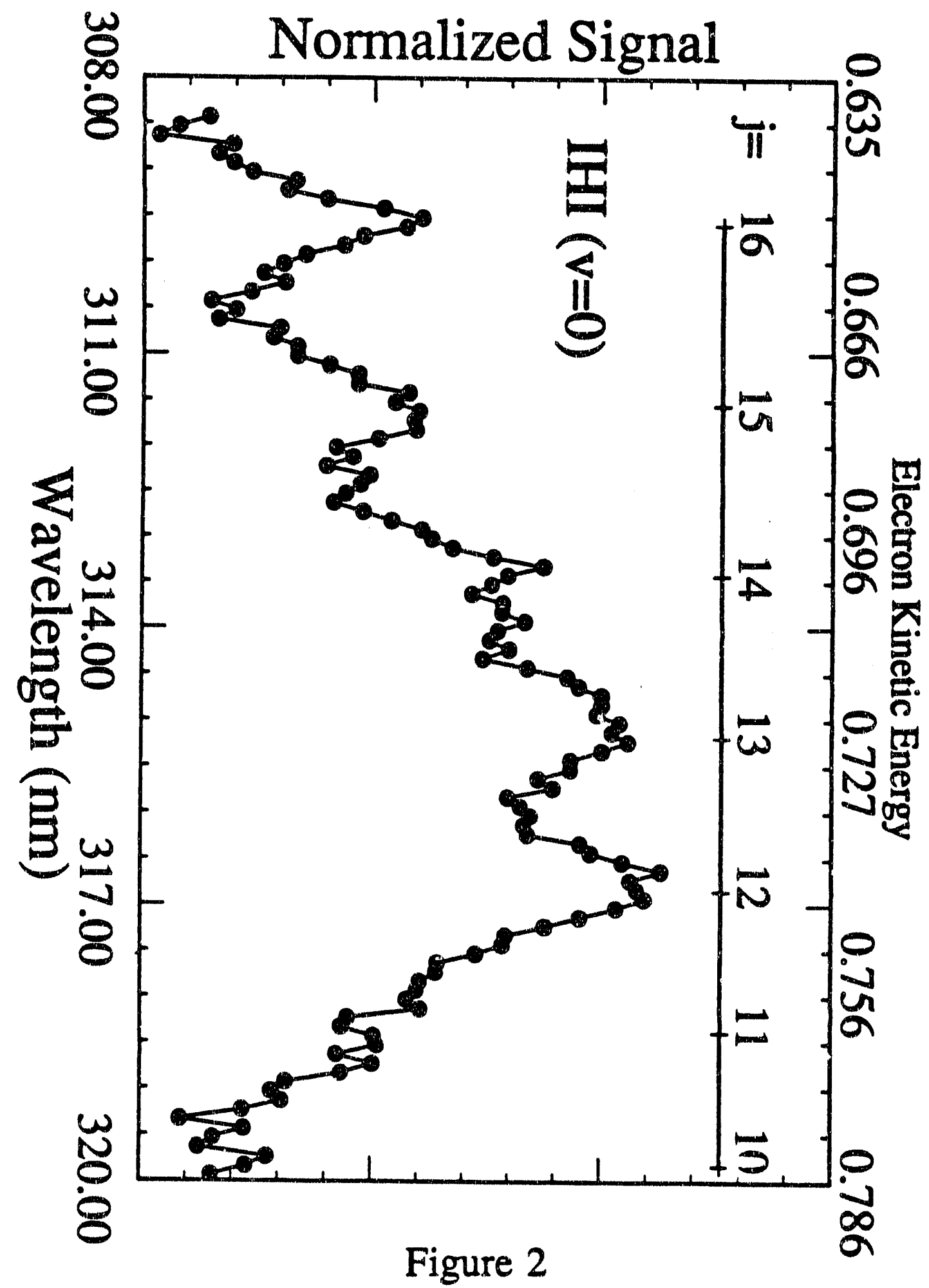




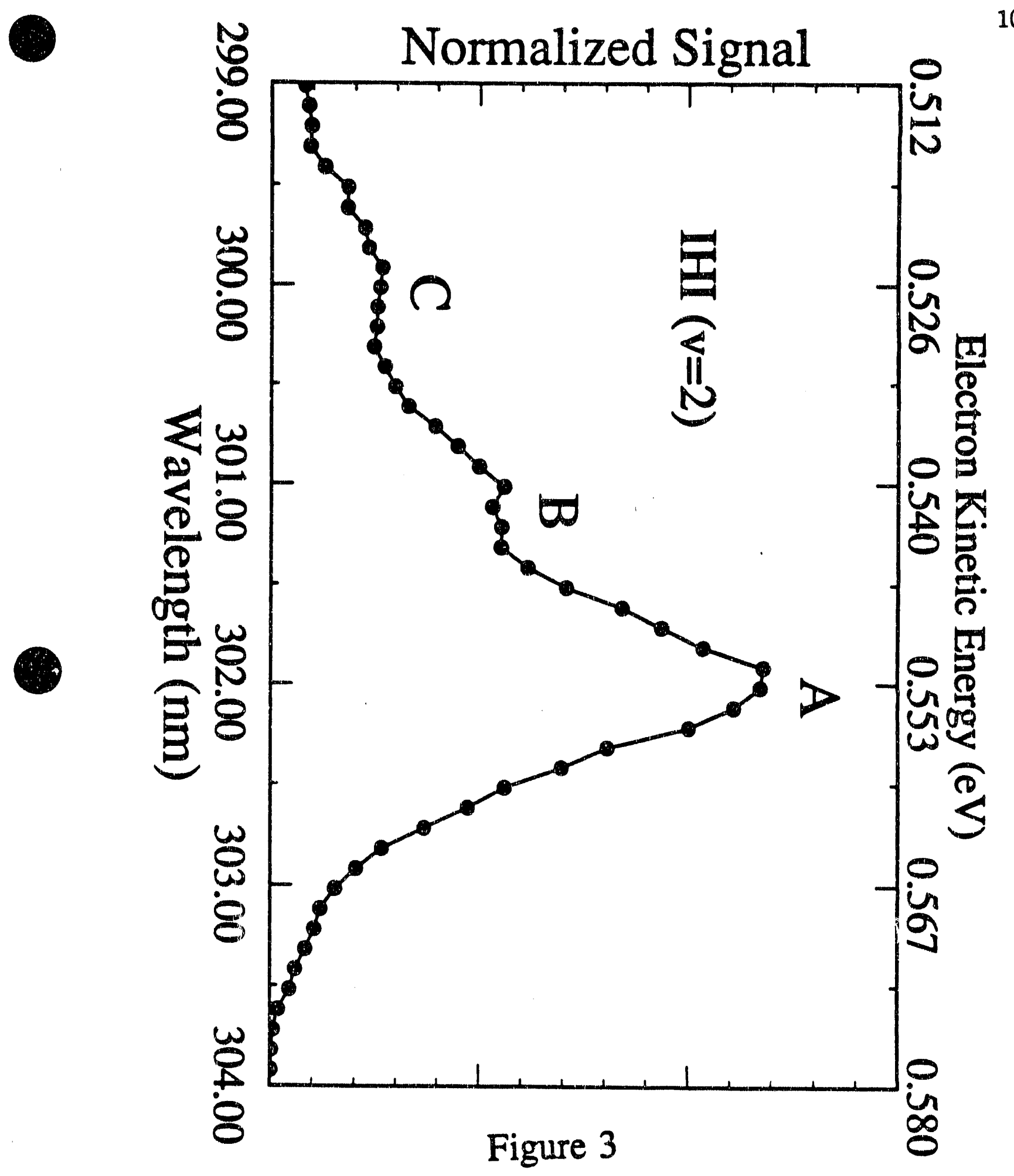




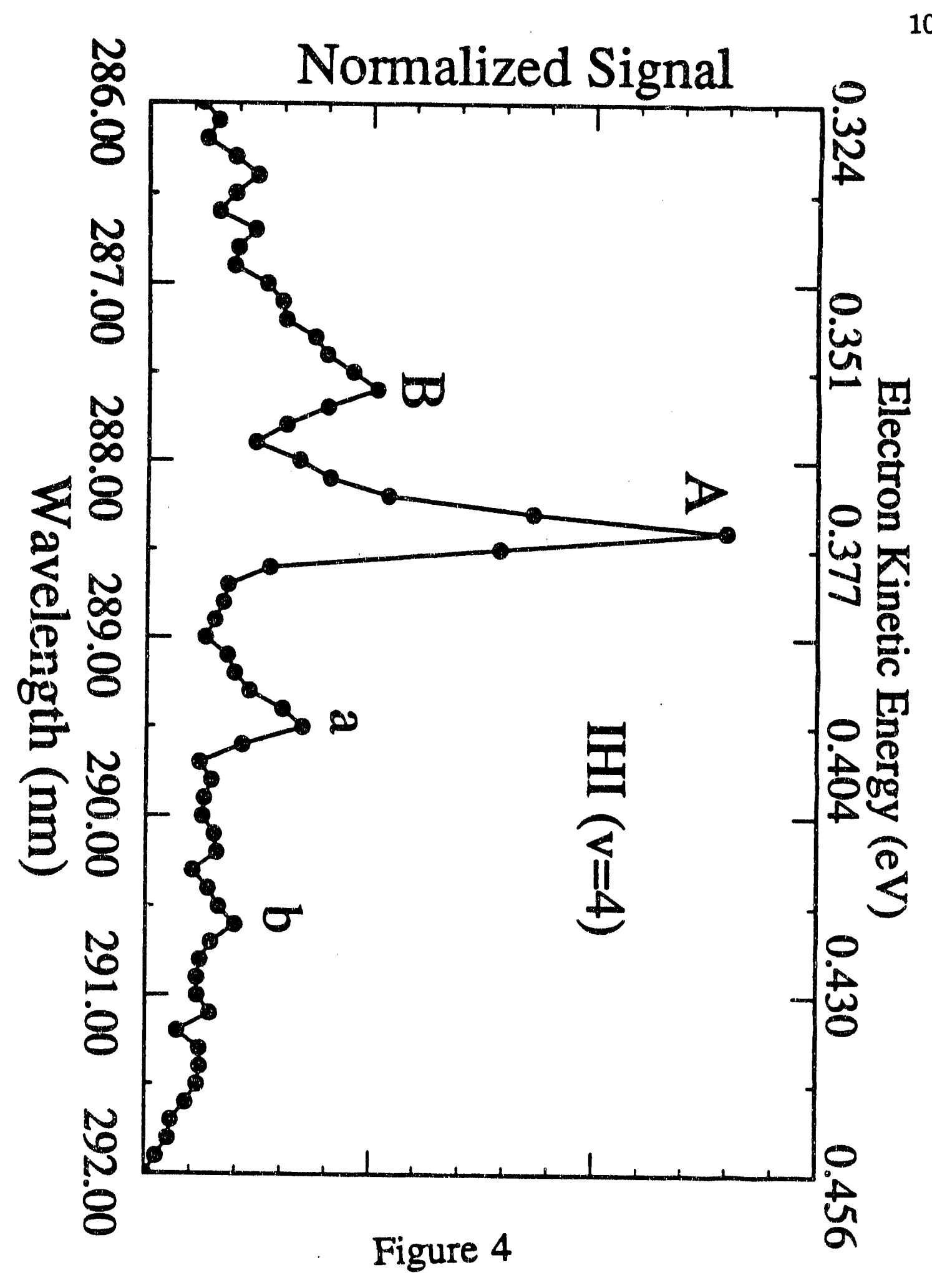

108 


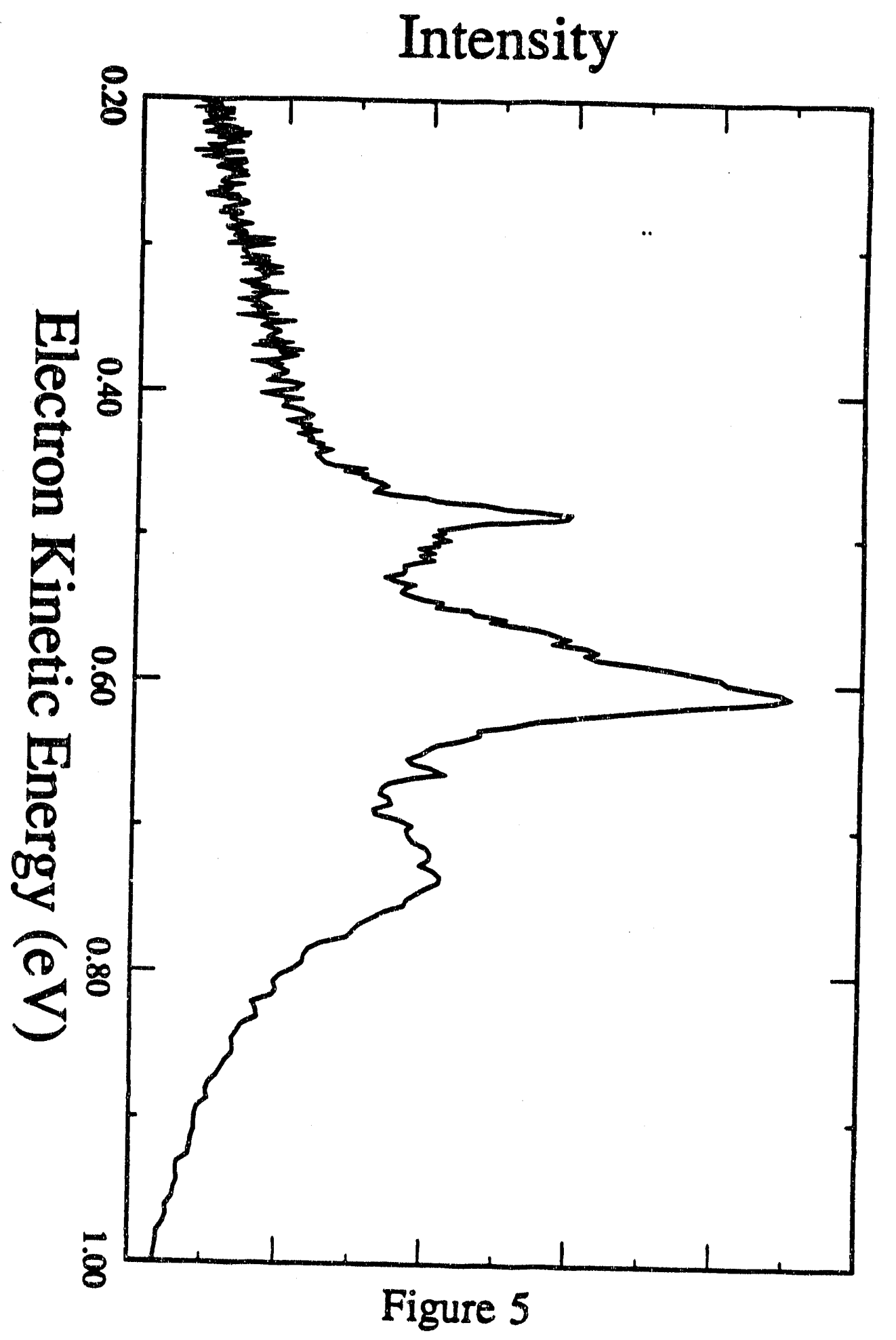




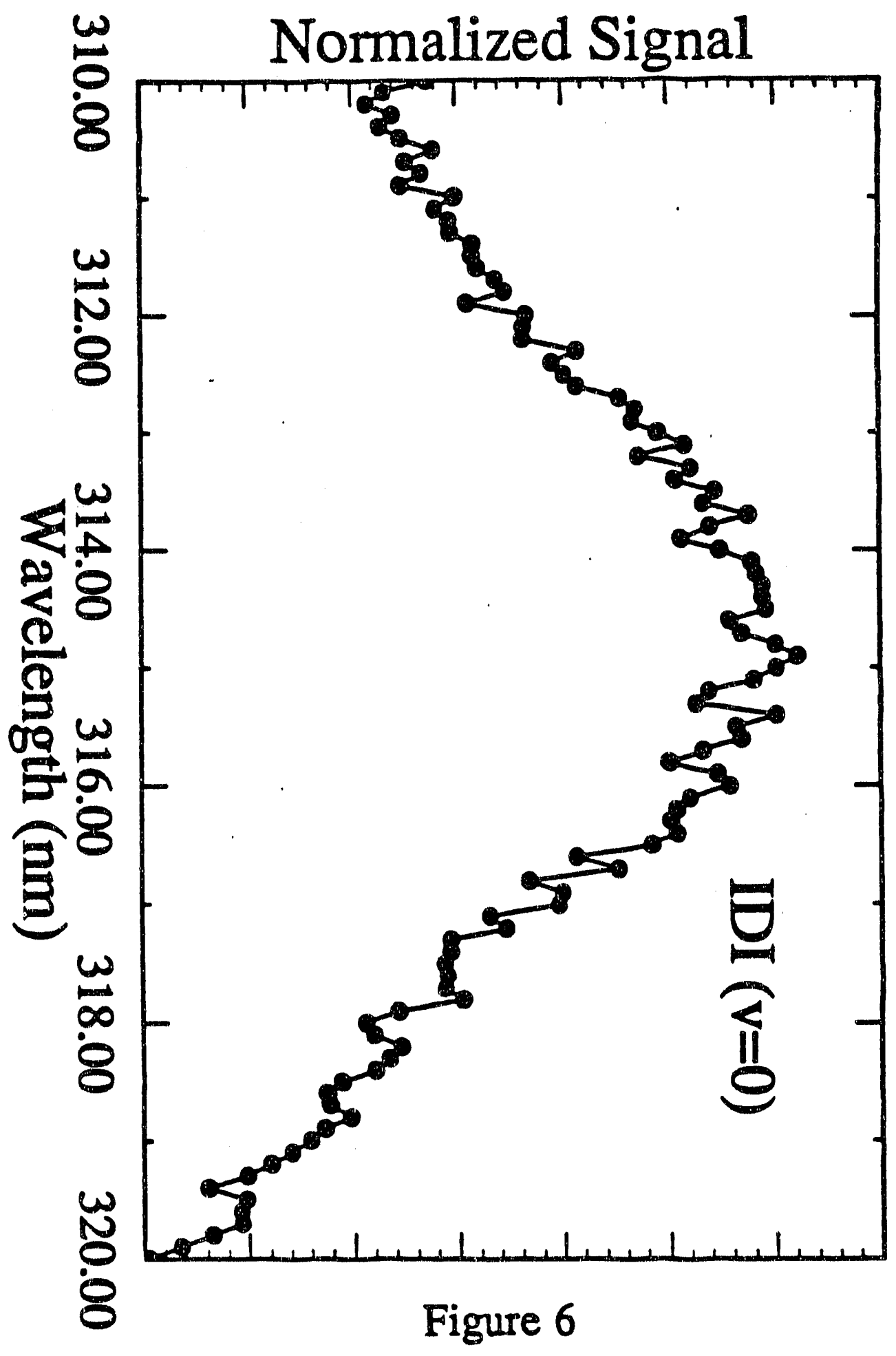




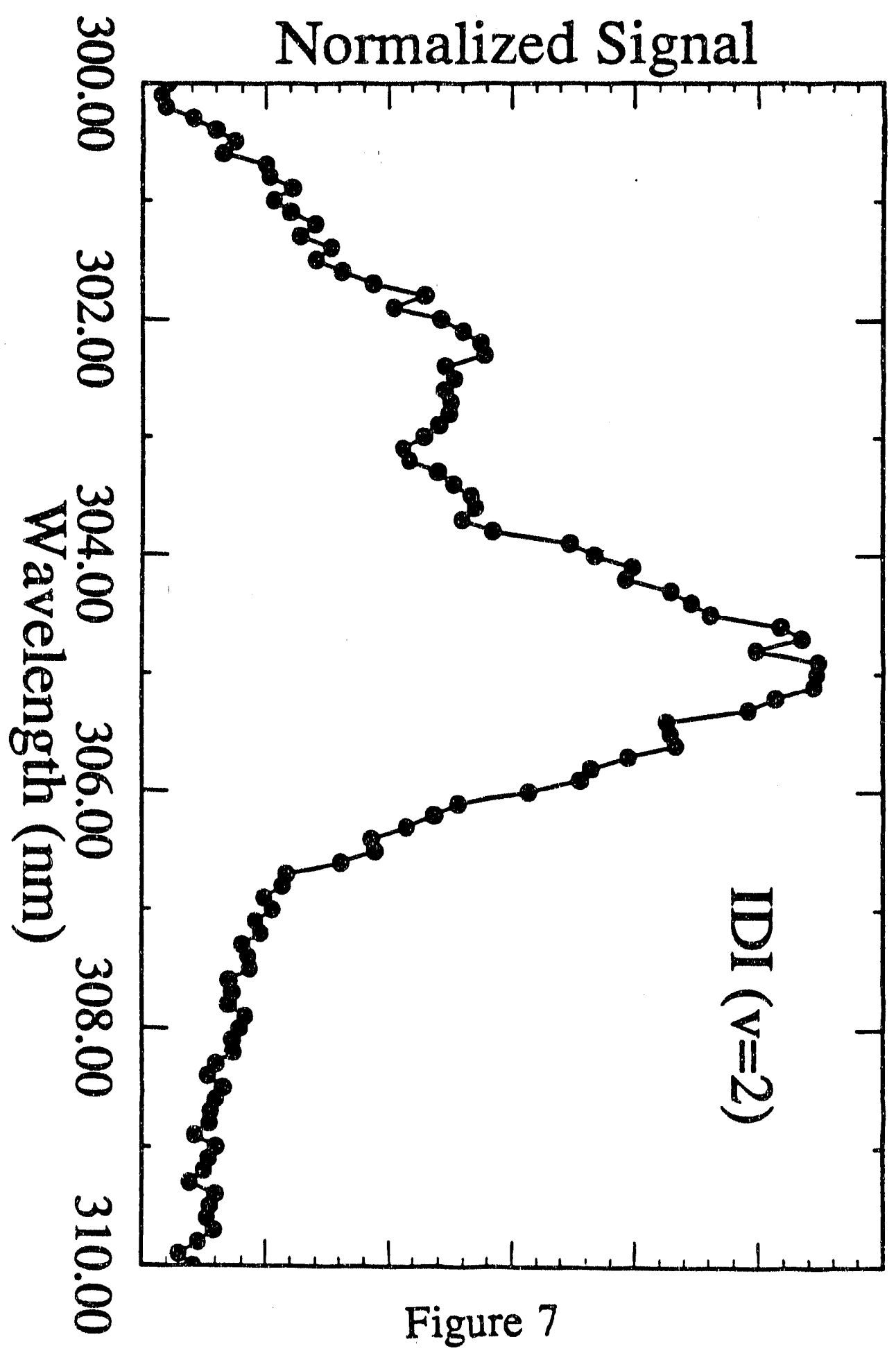




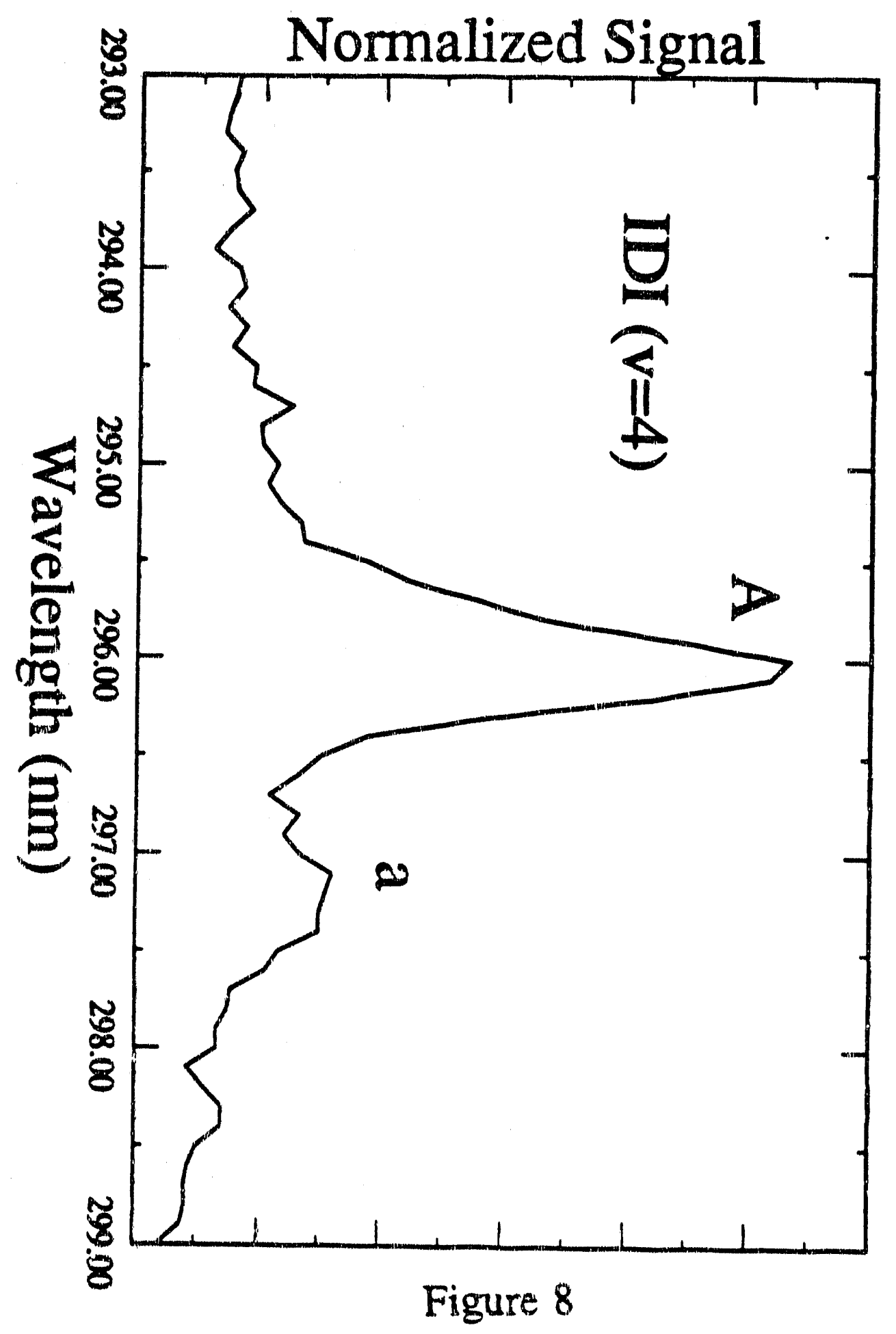




\section{References}

1. Metz, R.B.; Kitsopoulos, T; Weaver, A.; Neumark, D.M. J. Chem. Phys. 1988, 88, 1463. Metz, R.B.; Weaver, A.; Bradforth, S.E.; Kitsopoulos, T.N.; Neumark, D.M.; J. Phys. Chem. to appear 2/90.

2. Weaver, A.; Metz, R.B.; Bradforth, S.B.; Neumark, D.M. J. Phys. Chem. 1988, 92, 5558.

3. Kitsopoulos, T.N.; Waller, I.M.; Loeser, J.G.; Neumark, D.M. Chem. Phys. Lett. 1989 59,300

4. Bakker, J.M.B. J. Phys. E 1973, 6, 785 (1973). Bakker, J.M.B. J. Phys. E 1974, 7, 364.

5. Müller-Dethlefs, K.; Sander, M.; Schlag, E.W. Z. Naturforsch. 1984, 39a, 1089; Sander, M.; Chewter, L.A.; Müller-Dethlefs, K.; Schlag, E.W. Phys. Rev. A 1987, 36, 4543.

6. Derived from the laser photon energy $(4.66 \mathrm{eV})$, the electron affinity of Iodine ( 3.0591 eV) Hotop, H.; Linegerger, W.C. J.Phys. Chem. Ref. Data 1985, 14, 731, and the enthalpy of dissociation of $\mathrm{IHI}^{*}$ into $\mathrm{I}^{-}+\mathrm{HI}(\mathrm{v}=0)(0.74 \pm 0.13 \mathrm{eV})$ Caldwell, G; Kebarle, P. Can. J. Chem. 1985, 63, 1399.

7. Schatz, G.C. J. Chem. Phys. 1989, 90, 4847. Schatz, G.C. J. Chem. Phys. submitted.

8. Schatz, G.C. Israel J. Chem. 1989, 29, 361.

9. Schatz, G.C. J. Phys. Chem. 1990, 94, 6157.

10. Bowman, J.M.; Gazdy, B. J. Phys. Chem. 1989, 93, 5129.

11. Romelt, J. Chem. Phys. 1983, 79, 197; Bondi D.K., Connor J.N.L., Manz J., Romelt, J. Mol. Phys. 1983, 50, 467; Kaye J.A. and Kuppermann A., Chem. Phys. Lett. 1984, 88, 
2758; Schatz G.C. Chem. Phys. Lett. 1988 409.

12. Ault, B.S. Acc. Chem. Res. 1982, 15, 103. Ellison, C.M.B.; Ault, B.S. J. Phys. Chem. 1979, 83, 832.

13. Schatz, G.C. private communication. Bowman, J.M. private communication. Gertitschke, P.L.; Manz, J.; Römelt, J.; Schor, H.H.R. J. Chem. Phys. 1985, 83.

14. Schatz, G.C. J. Chem. Soc.-Fara. Disc. 1990, 86, 1729.

15. Caldwell G. and Kebarle P. Can. J. Chem. 1985, 63, 1399.

16. Schatz, G.C. Chem. Phys. Lett. 1988, 151, 409. Amaee, B.; Connor, J.N.L.; Whitehead, J.C.; Jakubetz, W.; Schatz, G.C. Fara. Disc. Chem. Soc. 1987, 84, 387.

Kornweitz, H.; Broida, M.; Persky, A. J. Phys. Chem.1989, 93, 251. Schatz, G.C. J. Chem. Phys. 1989, 90, 3582.

17. White, J.M.; Thompson, D.L. J. Chem. Phys. 1974, 61, 719. 


\section{Chapter IV}

\section{Study of the low-lying electronic states of $\mathrm{Si}_{2}$ and $\mathrm{Si}_{2}{ }^{-}$using negative ion photodetachment techniques}

\section{Introduction}

The spectroscopy of elemental clusters has become the focus of much experimental and theoretical research in recent years. ${ }^{1,2,3,4}$ A primary goal of these studies is to determine how chemical bonding in a cluster evolves as the number of atoms is varied. A full understanding of bonding in a cluster requires the characterization not only of its ground electronic state, but also of its low-lying excited electronic states. This is a formidable challenge due to the likely presence of a large number of low-lying electronic states in a many-atom cluster. ${ }^{5,6,7,8}$ As a first step towards understanding the nature of electronic states in larger clusters, we report here the results of two types of photodetachment experiments on $\mathrm{Si}_{2}{ }^{-}$which yield a detailed picture of the low-lying electronic states of $\mathrm{Si}_{2}$ as well as the lowest two electronic states of $\mathrm{Si}_{2}{ }^{-}$.

The electronic structure of $\mathrm{Si}_{2}$ is complex. It has three low-lying molecular orbital configurations: $(3 p) \sigma_{B}{ }^{2}(3 p) \pi_{u}{ }^{2}, \sigma_{g}{ }^{1} \pi_{u}{ }^{3}$ and $\sigma_{B}{ }^{0} \pi_{u}{ }^{4}$. The two triplet and four singlet states arising from these configurations are shown in Figure 1. Ab initio calculations predict the $\mathrm{X}^{3} \Sigma_{\mathrm{g}}\left(\sigma_{\mathrm{g}}{ }^{2} \pi_{\mathrm{u}}{ }^{2}\right)$ to be the ground state and the $\mathrm{D}^{3} \Pi_{\mathrm{u}}\left(\sigma_{\mathrm{g}}{ }^{1} \pi_{\mathrm{u}}{ }^{3}\right)$ state to lie only $20-100 \mathrm{meV}$ above, ${ }^{9,10,11,12,13,14}$ this interval is very sensitive to the level of the calculation. The $a^{1} \Delta_{g}, b^{1} \Pi_{v}$, and $c^{1} \Sigma_{g}^{+}$singlet states are predicted to lie between 0.4-0.9 eV above the ground state, with the $\mathrm{d}^{1} \Sigma_{\mathrm{g}}{ }^{+}$state slightly higher (1.1-1.5 eV). Experimentally, electronic transitions originating from the $X^{3} \Sigma_{8}^{\cdot}$ and $D^{3} \Pi_{u}$ states have been observed by several 
investigators, ${ }^{15,16}$ but transitions between the two states have not been seen. The only previous experimental value for the D-X splitting, $53 \pm 15 \mathrm{meV}$, was obtained from the $\mathrm{Si}_{2}-$ photoelectron spectroscopy work of Ellison and co-workers ${ }^{17}$. Within the singlet manifold, absorption bands originating from the $c^{1} \Sigma_{g}{ }^{+}$state have been observed, ${ }^{18}$ and an emission band due to the the $d^{1} \Sigma_{\varepsilon}^{+}-b^{1} \Pi_{u}$ transition has been seen. ${ }^{19}$ However, prior to the work reported here, the $a^{1} \Delta_{8}$ state had not been observed, the energy of the $c^{1} \Sigma_{g}^{+}$ state relative to the other low-lying singlet states was not known, and the energy of the entire manifold of singlet states relative to the triplet manifold had not been determined.

The silicon dimer anion $\mathrm{Si}_{2}$-also has two low-lying electronic states: the ${ }^{2} \Sigma_{B}{ }^{+}$state from the $\sigma_{\mathrm{B}} \pi_{\mathrm{u}}{ }^{4}$ configuration and the ${ }^{2} \Pi_{\mathrm{u}}$ state from the $\sigma_{\mathrm{B}}{ }^{2} \pi_{\mathrm{u}}{ }^{3}$ configuration. $A b$ initio calculations predict these states to be nearly degenerate..$^{20,21}$ If we assume that the ground electronic state $\mathrm{Si}_{2}$ - results from adding one electron to the LUMO of the $\mathrm{X}^{3} \Sigma_{\mathrm{g}}$. state of $\mathrm{Si}_{2}$ (the $\pi_{u}$ molecular orbital), we would expect the ${ }^{2} \Pi_{u}$ to be the ground state. Again, the only prior experimental probe of this splitting is Ellison's $\mathrm{Si}_{2}{ }^{-}$photoelectron spectrum; ${ }^{17}$ from his analysis, he concluded that the ${ }^{2} \Pi_{u}$ state is the ground state and the ${ }^{2} \Sigma_{\mathrm{g}}^{+}-{ }^{2} \Pi_{\mathrm{u}}$ splitting is $117 \pm 16 \mathrm{meV}$

In order to sort out the electronic states in $\mathrm{Si}_{2}{ }^{-}$and $\mathrm{Si}_{2}$, we have studied $\mathrm{Si}_{2}{ }^{-}$ using two forms of photodetachment spectroscopy: fixed-frequency photoelectron spectroscopy and threshold photodetachment spectroscopy. Negative ion photodetachment is a powerful method for studying clusters ${ }^{22,23,24,25,26}$ since one can easily massselect the species of interest prior to spectroscopic investigation. In addition, since the selection rules for photodetachment are different from those of optical spectroscopy, one 
can probe electronic states that are "dark" (forbidden) in emission or absorption experiments. ${ }^{27}$ In particular, all of the $\mathrm{Si}_{2}$ electronic states in Figure 1 are accessible from either the ${ }^{2} \Pi_{w}$ or ${ }^{2} \Sigma_{8}{ }^{+}$states of $\mathrm{Si}_{2}{ }^{-}$. The combination of the two experiments is quite powerful. Although the energy resolution of the threshold photodetachment spectrometer is considerably higher than that of the photoelectron spectrometer, not all of the allowed photodetachment transitions are observed in the threshold spectrometer (see below).

The two experiments have enabled us to determine the energies of the six $\mathrm{Si}_{2}$ electronic states in Figure 1, as well as the splitting between the two low-lying electronic states of the anion. We find that the $\mathrm{X}^{3} \Sigma_{\mathrm{g}}{ }^{+}$and ${ }^{2} \Pi_{\mathrm{u}}$ states are indeed the ground states of $\mathrm{Si}_{2}$ and $\mathrm{Si}_{2}{ }^{-}$, respectively. The $\mathrm{D}^{3} \Pi_{\mathrm{u}}-\mathrm{X}^{3} \Sigma_{\mathrm{B}}{ }^{+}$interval in $\mathrm{Si}_{2}$ is $83 \pm 10 \mathrm{meV}$, and the ${ }^{2} \Sigma_{B}^{+}-{ }^{2} \Pi_{u}$ interval in $\mathrm{Si}_{2}-$ is $24 \pm 10 \mathrm{meV}$. In addition, we determined spectroscopic constants (vibrational frequencies, bond lengths and spin orbit splittings) for both the triplet and singlet states of $\mathrm{Si}_{2}$, as well as for the ${ }^{2} \Sigma_{\mathrm{g}}{ }^{+}$and ${ }^{2} \Pi_{u}$ states of $\mathrm{Si}_{2}{ }^{-}$.

\section{Experimental}

Two instruments were used in this study: a time-of-flight (TOF) photoeiectron spectrometer ${ }^{28}$ and a threshold photodetachment spectrometer. ${ }^{29}$ In both instruments, $\mathrm{Si}_{2}-$ anions are generated by laser vaporization. The anions are mass-selected in a timeof-flight mass spectrometer. The anion of interest is subsequently photodetached and the resulting photoelectrons are detected using one of the schemes illustrated in Figure 2 and described in more detail below.

The silicon negative ion clusters in both spectrometers are generated with a laser 
vaporization/pulsed molecular beam source. ${ }^{30}$ The output of a $\mathrm{XeCl}$ excimer laser (308 $\mathrm{nm}, 5-15 \mathrm{~mJ} / \mathrm{pulse}$ ) is focused onto the surface of a rotating and translating silicon rod. The resulting plasma is entrained in a pulse of He from a pulsed solenoid valve $(0.05 \mathrm{~cm}$ diameter orifice), and expanded through a "clustering channel" into the source vacuum chamber. This arrangement produces negative ions in sufficient quantity directly; no further means of attaching electrons to neutral clusters is needed.

In the photoelectron spectrometer, a Wiley-McLaren time-of-flight mass spectrometer is used for mass separation. ${ }^{31}$ The anions are extracted from the source region by applying a pulsed $100 \mathrm{~V} / \mathrm{cm}$ field perpendicular to the molecular beam axis. The anions are accelerated to $1000 \mathrm{eV}$, and the ions separate into bunches according to mass as they traverse a $140 \mathrm{~cm}$ flight tube. In the threshold photodetachment spectrometer, the cluster beam passes through a $2 \mathrm{~mm}$ skimmer into a lower pressure, differentially pumped region before any acceleration occurs. The anions are accelerated to $1000 \mathrm{eV}$ and mass-selected using a time-of-flight mass spectrometer based on Bakker's design. ${ }^{32}$ Based on the observed 'hot bands' (see below), the anions produced in the threshold spectrometer source were colder than in the photoelectron spectrometer source. This may have resulted from not applying any acceleration fields in the relatively high pressure source region in the threshold spectrometer, but may also be due to slightly different source operating conditions used in the two instruments. $\quad$ I $n \quad t h$ e photoelectron spectrometer, the mass-selected $\mathrm{Si}_{2}{ }_{2}$ anions are photodetached with a fixedfrequency pulsed laser. A small fraction $\left(10^{-4}\right)$ of the ejected photoelectrons are collected at the end of a $100 \mathrm{~cm}$ field-free flight tube and are energy-analyzed by time of flight. 
The electron kinetic energy distribution yields the transition energies between the anion and neutral (Figure 2, top). The energy resolution of this instrument is $8 \mathrm{meV}\left(64 \mathrm{~cm}^{-1}\right)$ for electrons with $0.65 \mathrm{eV}$ kinetic energy and degrades as $(\mathrm{KE})^{3 / 2}$. This is sufficient to resolve vibrational features in the photoelectron spectrum. The spectra here were obtained with the third harmonic of a Nd:YAG laser at $355 \mathrm{~nm}, 20 \mathrm{~Hz}$ repetition rate and each spectrum was signal averaged for 80,000 laser shots. Spectra were obtained at two polarization angles, $\theta=0^{\circ}$ and $90^{\circ}$, where $\theta$ is the angle between the electric vector of the laser light and the direction of electron detection.

In the threshold photodetachment spectrometer, $\mathrm{Si}_{2}{ }^{-}$was photodetached using an excimer-pumped tunable dye laser operating at $50 \mathrm{~Hz}$. Only those photoelectrons with nearly zero kinetic energy are collected (Figure 2, bottom). In principle, this yields the same information as photoelectron spectroscopy. However, the energy resolution is considerably higher. This particular threshold electron detection scheme was developed by Müller-Dethlefs et al. for neutral photoionization. ${ }^{33}$ Our implementation of this to threshold photodetachment of anions has been described previously. ${ }^{29}$ Gantenfor et al. ${ }^{34}$ have recently used threshold photodetachment to study $\mathrm{Au}_{2}-$. The resolution of our instrument is $3 \mathrm{~cm}^{-1}$ at best, but for optimum signal-to-noise, the threshold spectra in this work were obtained at an estimated resolution of $15 \mathrm{~cm}^{-1}$. At this resolution, we observe spin-orbit fine structure in addition to vibrational suructure, but individual rotational transitions are not resolved.

The threshold spectra were signal averaged for 1000 laser shots at each laser frequency. In order to probe the spectral region of interest (435 nm to $595 \mathrm{~nm}$ ), four 
different laser dyes were used : Coumarin 440 (423 nm - 460 nm), Coumarin 460 (440 $\mathrm{nm}-480 \mathrm{~nm})$, Coumarin $540(530 \mathrm{~nm}-580 \mathrm{~nm})$, and Rhodamine 6G $(580 \mathrm{~nm}-600 \mathrm{~nm})$.

\section{Results}

Our photoelectron spectra obtained at $355 \mathrm{~nm}(\mathrm{hv}=3.49 \mathrm{eV})$ and $\theta=0^{\circ}$ and $90^{\circ}$ are presented in Figures 3 and $4 .^{35}$ The electron kinetic energy (eKE) is related to the internal (vibrational + electronic) energy of the anion $\mathrm{E}^{(-)}$and the neutral $\mathrm{E}^{(0)}$ by

$$
\mathrm{eKE}=\mathrm{h} v-\mathrm{EA}-\mathrm{E}^{(0)}+\mathrm{E}^{(-)}
$$

where EA is the electron affinity of the neutral ground state. Peaks A-F are in the same energy range as the peaks seen by Ellison ${ }^{17}$ and correspond to transitions to the triplet states of $\mathrm{Si}_{2}$, while peaks G-L are due to transitions to higher lying singlet states.

Figures 3 and 4 show that varying the laser polarization changes the peak intensities in different ways. Peaks $D$ and $E$ are more intense at $\theta=0^{\circ}$ than at $90^{\circ}$, peaks $\mathrm{B}, \mathrm{C}$ and $\mathrm{F}$ are relatively insensitive to the laser polarization, while peaks $\mathrm{d}$ and $\mathrm{e}$ are not evident in the $\theta=0^{\circ}$ spectrum. The singlet band also shows three types of peaks. Peaks $\mathrm{G}, \mathrm{H}$ and $\mathrm{J}$ have maximum intensity at $\theta=0^{\circ}, I$ and $\mathrm{K}$ are most intense at $\theta=90^{\circ}$, and peak $\mathrm{L}$ has the same intensity in both polarizations. In Ellison's spectrum, a lower photon energy was used $(2.54 \mathrm{eV})$ and thus only four peaks (A-D) were observed. In addition, the D,d doublet in Figure 4 was not resolved.

The threshold photodetachment spectra are shown in Figures 5 and 6 . Several of the peaks in the photoelectron spectra appear as multiple peaks in the higher resolution threshold spectra. In the spectrum of Figure 5 , peak $B$ becomes a doublet $\left(B_{1}, B_{2}\right)$, while peak $C$ splits into $C_{1}$ and $C_{2}$ along with additional weak peaks labeled $c_{1}{ }^{\prime}$ and $c_{3}{ }^{\prime}$. In 
addition, peak $d$ appears as three intense transitions $\left(d_{1}, d_{2}, d_{3}\right)$ and a small peak $c_{2}$; peak e also becomes a triplet $\left(e_{1}, e_{2}, e_{3}\right)$. Peaks $D$ and $E$ in the photoelectron spectra are absent from the threshold spectrum. The significance of this observation will be discussed below.

Similar effects are observed for the singlet band. Peaks $\mathrm{G}$ and $\mathrm{H}$ become doublets at higher resolution, peak $\mathrm{J}$ remains a single peak, and peaks I and $\mathrm{K}$ are absent from the threshold spectrum. The peak positions for all the spectra are listed in Table 1. In all the threshold spectra, the peak widths are about $40 \mathrm{~cm}^{-1}(5 \mathrm{meV})$. This is most likely a combination of instrumental resolution and broadening from unresolved rotational transitions.

\section{Discussion}

In many small molecule photoelectron spectra, it is straightforward to distinguish transitions between different electronic states of the anion and neutral from vibrational progressions within a given electronic band. ${ }^{36}$ However, the predicted spacings between several of the $\mathrm{Si}_{2}$ and $\mathrm{Si}_{2}$ - low-lying electronic states are comparable to the known and calculated vibrational frequencies, most of which are around $500 \mathrm{~cm}^{-1}(60 \mathrm{meV})$. Thus, the key to understanding the $\mathrm{Si}_{2}-$ photoelectron spectra and threshold photodetachment spectra is to be able to sort out the overlapping electronic transitions and vibrational progressions in these spectra.

The polarization dependence of the peaks in a photoelectron spectrum ${ }^{37}$ provides a means of distinguishing electronic from vibrational transitions. As described in the Results, the sets of peaks $(B, C, F),(d, e)$, and $(D, E)$ in the triplet region of the 
photoelectron spectra each have different angular distributions with respect to the electric field vector of the laser, suggesting that each set is due to a different neutral $\leftarrow$ anion electronic transition. The spacing between peaks $d$ and $e$ is identical to that between $D$ and $\mathrm{E}\left(64 \mathrm{meV}\right.$ or $\left.516 \mathrm{~cm}^{-1}\right)$, implying that each pair is a vibrational progression in the same electronic state of neutral $\mathrm{Si}_{2}$. Similarly, in the singlet region, peaks $(\mathrm{G}, \mathrm{H}, \mathrm{J})$, peaks $(\mathrm{I}, \mathrm{K})$, and peak $\mathrm{L}$ apparently arise from three different electronic transitions.

We now consider the transitions between $\mathrm{Si}_{2}{ }^{-}$and $\mathrm{Si}_{2}$ electronic states that could contribute to the triplet regich of the spectra. The experimental values of $r_{e}$, the equilibrium bond length, and $\omega_{\mathrm{e}}$, the harmonic vibrational frequency, are $2.246 \AA$ and 511 $\mathrm{cm}^{-1}$ for the $\mathrm{Si}_{2} \mathrm{X}^{3} \Sigma_{\mathrm{g}} \cdot$ state and $2.155 \AA, 539 \mathrm{~cm}^{-1}$ for the $D^{3} \Pi_{\mathrm{u}}$ state. ${ }^{15,38}$ Calculations by Bauschlicher yield a $D^{3} \Pi_{u}-X^{3} \Sigma_{B} \cdot$ gap of $440 \pm 100 \mathrm{~cm}^{-1}(55 \pm 12 \mathrm{meV})$. Other calculations by $B_{r u n a}{ }^{11}$ and McLean ${ }^{12}$ predict splittings of $20 \mathrm{meV}$ and $130 \mathrm{meV}$, respectively. In spite of the numerical disagreement surrounding this criplet gap, the consensus among theoreticians is that the $\mathrm{X}^{3} \Sigma_{\mathrm{B}}$ state is the ground state of the neutral dimer.

In a recent calculation by Raghavachari and Rohlfing ${ }^{21}$ on $\mathrm{Si}_{2}-\mathrm{r}_{\mathrm{e}}$ and $\omega_{\mathrm{e}}$ were found to be $2.124 \AA$ and $579 \mathrm{~cm}^{-1}$ for the ${ }^{2} \Sigma_{\mathrm{g}}{ }^{+}$state and $2.202 \AA, 539 \mathrm{~cm}^{-1}$ for the ${ }^{2} \Pi_{v}$ state. They also found the ${ }^{2} \Pi_{\mathrm{u}}$ state to lie $43 \mathrm{meV}$ below the ${ }^{2} \Sigma_{\mathrm{g}}{ }^{+}$state. However, as was the case in an earlier calculation by Bruna, ${ }^{20}$ the splitting and even the ordering of these states is uncertain.

In Figure 7, we have drawn an energy level diagram for the first two electronic states of the neutral and anion dimer. The ordering of the levels is based on the above $a b$ initio results. In a typical photoelectron spectrum, one only observes transitions 
between anion and neutral electronic states which involve removal of only one electron (one-electron transitions). According to this rule, three of the four possible transitions among these states are allowed; only the $X^{3} \Sigma_{\mathrm{g}} \leftarrow^{2} \Sigma_{\mathrm{B}}{ }^{+}$transition is forbidden. The three allowed transitions are labelled [R], [S] and [T] corresponding to the $D^{3} \Pi_{u} \leftarrow{ }^{2} \Pi_{u}, X^{3} \Sigma_{B}$. $\leftarrow{ }^{2} \Pi_{v}$ and $D^{3} \Pi_{u} \leftarrow{ }^{2} \Sigma_{g}{ }^{+}$transitions, respectively, where we have maintained the same notation used by Ellison. ${ }^{17}$ Thus, Figure 7 explains why three overlapping electronic transitions occur in the triplet region of the photoelectron spectrum. However, a unique identification of the peaks in the spectra with the three allowed transitions requires additional information obtained from the threshold photodetachment spectra.

The first point to consider is the reason why peaks $D$ and $E$ are absent from the threshold photodetachment spectra. The energy dependence of the photodetachment cross section near the threshold energy $E_{L h}$ for an neutral $\leftarrow$ anion transition is described by Wigner's law, ${ }^{39}$

$$
\sigma(E) \propto\left(E-E_{\mathrm{th}}\right)^{t+1 / 2}
$$

where $\ell$ is the orbital angular momentum of the detached electron. For $s$-wave $(\ell=0)$ detachment, the cross section goes as $\left(E-E_{t h}\right)^{1 / 2}$ and is therefore quite large just above the threshold energy. However, for $\ell \geq 1$ ( $p$-wave higher), $\sigma(E)$ is very small just above $E_{\mathrm{bh}}$. In our threshold photodetachment spectrometer, only those photoelectrons produced a few $\mathrm{cm}^{-1}$ above an neutral $\leftarrow$ anion transition are collected. Thus, only transitions that proceed via s-wave detachment are observed.

Reed et al. $^{40}$ have shown that one can predict if s-wave photodetachment can 
occur based on the symmetry of the molecular orbital in the anion from which photodetachment occurs. For $\mathrm{Si}_{\lambda}-$, which has $\mathrm{D}_{\text {cob }}$ symmetry, photodetachment from a $\pi_{u}$ orbital can go by an s-wave near threshold, whereas s-wave photodetachment from a $\sigma_{8}$ orbital is forbidden and only detachment via higher partial waves $(\geq 1)$ can occur. Figure 7 shows that the electronic transitions $[S]$ and $[T]$ involve the removal of a $\pi_{u}$ electron can therefore undergo s-wave photodetachment. On the other hand, in transition [R], a $\sigma_{8}$ electron is ejected and s-wave detachment cannot occur. Hence, the absence of peaks $D$ and $E$ in the threshold photodetachment spectrum means they are due to the electronic transition $[R]$, the $D^{3} \Pi_{u} \leftarrow{ }^{2} \Pi_{u}$ transition. Peaks $D$ and $E$ are separated by 516 $\pm 60 \mathrm{~cm}^{-1}$ in the photoelectron spectra, which is characteristic of a vibrational frequency in either $\mathrm{Si}_{2}$ or $\mathrm{Si}_{2}-$. The ab initio calculations discussed above predict a relatively small bond length change upon photodetachment, so the considerably greater intensity of peak $\mathrm{D}$ indicates it is the $v^{\prime}=0 \leftarrow v^{\prime \prime}=0$ vibrational transition associated with the electronic transition $[R]$, while $E$ is the $1 \leftarrow 0$ vibrational transition.

We can now assign the peaks that appear in the threshold photodetachment spectra. Consider the $d$ and e peaks in the spectrum of Figure 5. At the higher resolution in this spectrum, both peaks appear as triplets, with the peaks in each triplet evenly spaced by about $64 \pm 10 \mathrm{~cm}^{-1}$. This compares well with the experimentally determined $71.6 \mathrm{~cm}^{-1}$ splitting between the ${ }^{3} \Pi_{2},{ }^{3} \Pi_{1},{ }^{3} \Pi_{0}$ spin-orbit components (listed in order of ascending energy) of the ${ }^{3} \Pi_{u}$ electronic state. ${ }^{15}$ This triplet pattern is expected for the $\mathrm{D}^{3} \Pi_{u} \leftarrow{ }^{2} \Sigma_{r}^{+}$reutral $\leftarrow$ anion transition, (the spin-rotation splitting in the ${ }^{2} \Sigma_{\mathrm{g}}^{+}$will not be resolved in our spectra) and peaks $d$ and e are assigned to this electronic transition ([T] 
in Figure 7). The $d_{i}-e_{i}$ separation is $530 \pm 10 \mathrm{~cm}^{-1}$, and, based on the much greater intensity of $d$, peaks $d$ and $e$ are assigned to the $0 \leftarrow 0$ and $1 \leftarrow 0$ vibrational transitions associated with [T].

Peaks B and C each appear as doublets split by $122 \pm 10 \mathrm{~cm}^{-1}$ in Figure 5. The only unassigned neutral $\leftarrow$ anion transition in Figure 7 is $[S]$, the $X^{3} \Sigma_{g} \cdot \leftarrow{ }^{2} \Pi_{u}$ transition. This transition should yield doublets separated by the spin-orbit splitting between the ${ }^{2} \Pi_{3 / 2}$ and ${ }^{2} \Pi_{1 / 2}$ levels of the anion. We therefore assign peaks $B$ and $C$ to this transition and the observed $122 \mathrm{~cm}^{-1}$ interval to the spin-orbit splitting in the anion ${ }^{2} \Pi_{u}$ state. Based on the ordering of the ${ }^{3} \Pi_{\Omega}$ levels of $S i_{2}$, the ${ }^{2} \Pi_{3 / 2}$ level should be lower in energy. Peaks $B_{1}$ are a factor of 15 less intense than peaks $C_{i}$, so we assign peak $C$ to the $0 \rightarrow 0$ vibrational transition and peak $B$ to the $v^{\prime}=0 \leftarrow v^{\prime \prime}=1$ 'hot band' transition. This yields a vibrational frequency of $539 \pm 10 \mathrm{~cm}^{-1}$ for the anion ${ }^{2} \Pi_{u}$ state. The electron affinity of $\mathrm{Si}_{2}$ is given by the energy of peak $\mathrm{C}_{1}, 2.176 \pm 0.002 \mathrm{eV}$.

There are several additional small peaks in Figure 5 that we can assign. Peak $c_{2}$ lies $516 \mathrm{~cm}^{-1}$ above peak $C_{2}$. This is very close to the vibrational frequency of the $\mathrm{X}^{3} \Sigma_{\mathbf{g}}$ state of $\mathrm{Si}_{2}\left(v=509 \mathrm{~cm}^{-1}\right)$, and peak $c_{2}$ is therefore assigned to the $\mathrm{X}^{3} \Sigma_{\varepsilon}^{\cdot}\left(\mathrm{v}^{\prime}=1\right) \leftarrow{ }^{2} \Pi_{1 / 2}$ $\left(v^{\prime \prime}=0\right)$ transition. The partially resolved peaks $c_{1}{ }^{\prime}$ and $c_{3}{ }^{\prime}$ lie $528 \mathrm{~cm}^{-1}$ to the red of peaks $d_{1}$ and $d_{3}$ and are separated by the same interval. Peaks $c_{1}{ }^{\prime}$ and $c_{3}$ ' are therefore assigned to the $D^{3} \Pi_{u}\left(v^{\prime}=0\right) \leftarrow{ }^{2} \Sigma_{g}^{+}\left(v^{\prime \prime}=1\right)$ hot band transition, yielding $v_{01}=528 \pm 10$ $\mathrm{cm}^{-1}$ for the anion ${ }^{2} \Sigma_{g}{ }^{+}$state.

It is now clear that the energy level diagram of Figure 7 is qualitatively correct. From our assignments, we conclude that the ${ }^{3} \Pi_{\mathrm{u}}-{ }^{3} \Sigma_{\mathrm{g}} \cdot$ gap in $\mathrm{Si}_{2}$ is $83 \pm 10 \mathrm{meV}(669 \pm 80$ 
$\mathrm{cm}^{-1}$ ) which is higher than Bauschlicher's prediction' but lower than in McLean's calculation. ${ }^{12}$ For the anion, the ${ }^{2} \Pi_{u}$ state is established as the ground state and the ${ }^{2} \Sigma_{i}^{+}$${ }^{2} \Pi_{u}$ splitting is only $25 \pm 10 \mathrm{meV}\left(202 \pm 80 \mathrm{~cm}^{-1}\right)$. This is somewhat smaller than the values calculated by $\mathrm{Bruna}^{20}$ and Raghavachari. ${ }^{21}$

The discussion so far has centered on peak positions. We obtain additional information from the peak intensities, namely, the bond lengths in the anion electronic states and the anion vibrational temperature. This is possible because the bond lengths and frequencies for both the $X^{3} \Sigma_{B}$ and $D^{3} \Pi_{u}$ neutral states are known and the frequencies for the two anion states have been determined by our experiment. Consider first the $D^{3} \Pi_{v}$ $\leftarrow{ }^{2} \Sigma_{i}{ }^{+}$transition. Peaks $e_{i}, d_{i}$, and $c^{\prime}{ }_{i}$ are nominally the $1 \leftarrow 0,0 \leftarrow 0$, and $0 \leftarrow 1$ vibrational transitions associated with this electronic transition. In the absence of overlapping vibrational transitions, the $e_{1} / d_{i}$ intensity ratio is proportional to the ratio of Franck-Condon factors for the $1 \leftarrow 0$ and $0 \leftarrow 0$ transitions, one could therefore extract the bond length in the anion ${ }^{2} \Sigma_{B}+$ state from the $e_{i} / d_{i}$ ratio (assuming harmonic potentials throughout), and then obtain the anion vibrational temperature from the $c^{\prime} / d_{i}$ ratio.

In practice, some iteration is required in carrying out this procedure because the $2 \leftarrow 1$ and $1 \leftarrow 1$ sequence bands overlap the $1 \leftarrow 0$ and $0 \leftarrow 0$ transitions. Thus, in simulations of the spectrum, the $e_{/} / d_{i}$ intensity ratio also depends on the assumed value of the anion vibrational temperature. In addition, peaks $c^{\prime}$, and $c^{\prime}{ }_{3}$ are only partially resolved, so it is difficult to accurately determine their intensities. Our analysis yields a bond length of $2.116 \pm 0.005 \AA$ for the anion ${ }^{2} \Sigma_{\varepsilon}{ }^{+}$state and an approximate vibrational semperature of $500 \mathrm{~K}$. A similar analysis of the $X^{3} \Sigma_{8} \leftarrow{ }^{2} \Pi_{u}$ transition, using the known 
bond length of the $X^{3} \Sigma_{g}$ state and the $c_{2} / C_{2}$ intensity ratio gives $r_{e}=2.207 \pm 0.005 \AA$ for the ${ }^{2} \Pi_{u}$ state. This value of $r_{e}$ along with a vibrational temperature of $500 \mathrm{~K}$ yields reasonable intensities for peaks $B_{1}$ and $B_{2}$. Our geometries for both the ${ }^{2} \Sigma_{8}^{+}$and the ${ }^{2} \Pi_{u}$ states agree very well with $a b$ initio results on $\mathrm{Si}_{2}-21$ Figure 5 shows a simulation of the triplet band which includes all of the results discussed above.

From the $C_{1} / C_{2}$ (and $B_{1} / B_{2}$ ) ratio, we determine the ${ }^{2} \Pi_{1 / 2}{ }^{2} \Pi_{3 / 2}$ population ratio to be $1 / 2$, yielding a 'spir-orbit temperature' of $125 \mathrm{~K}$. Assuming the 'etachment cross sections out of the two spin-orbit states are the same, this result indicates that cooling of the spin-orbit states is more efficient than vibrational cooling in the cluster beam. This is a reasonable result as the energy interval is smaller for the spin-orbit states. All anion and neutral spectroscopic constants determined are summarized in Tables 2 and 3.

The anion properties determined from the analysis of the triplet band enable the assignment of the higher energy transitions to the singlet states of $\mathrm{Si}_{2}$. Figure 8 shows the one-electron transitions that can occur between the two anion electronic states and the $a^{1} \Delta_{g}, b^{2} \Pi_{u}, c^{1} \Sigma_{g}^{+}$, and $d^{1} \Sigma_{B}^{+}$states of $S i_{2}$. In Figure 10, we assume the dominant configuration for the $\mathrm{c}^{1} \Sigma_{\mathrm{g}}{ }^{+}$state to be the closed shell $\pi_{\mathrm{u}}{ }^{4}$ configuration and that for the $\mathrm{d}^{2} \Sigma_{\mathrm{g}}{ }^{+}$state to be the $\sigma_{\mathrm{g}}{ }^{2} \pi_{u}{ }^{2}$ open shell configuration (see below). Only the $\mathrm{a}^{\mathrm{a}} \Delta_{\mathrm{g}} \leftarrow{ }^{2} \Pi_{u}$, $b^{\prime} \Pi_{u} \leftarrow{ }^{2} \Sigma_{u}^{+}$, and $d^{1} \Sigma_{g}^{+} \leftarrow{ }^{2} \Pi_{u}$ neutral $\leftarrow$ anion transitions can ocsur by s-wave detachment and be observed in the threshold spectra. However. the $\mathrm{d}^{1} \Sigma_{\mathrm{g}}^{+}$state is predicted to lie above the energy range of both photodetachment spectra. ${ }^{11,12}$

In all transitions to singlet states of the neutral, any doublet peaks observed in the threshold spectrum must represent transitions from the ${ }^{2} \Pi_{v}$ state of the anion. We 
therefore assign the three equally spaced doublets between $480 \mathrm{~nm}$ and $454 \mathrm{~nm}$ in Figure 6 to a vibrational progression in the $a^{1} \Delta_{8} \leftarrow{ }^{2} \Pi_{u}$ electronic transition. The spacing between the doublets, $480 \pm 10 \mathrm{~cm}^{-1}$, is the vibrational frequency of the $\mathrm{a}^{1} \Delta_{8}$ sta., while the splitting within each doublet is identical to the spin-orbit splitting in the anion ${ }^{2} \Pi_{u}$ state determined from the triplet transitions. The $v^{\prime}=0 \leftarrow v^{\prime \prime}=0$ and $1 \leftarrow 0$ doublets correspond to peaks $\mathrm{G}$ and $\mathrm{H}$, respectively, in the photoelectron spectrum, while the smaller $2 \leftarrow 0$ doublet is not resolved in the photoelectron spectrum. This set of peaks represents the most extended vibrational progression seen in these spectra. Using the $2.216 \AA$ bond length for the anion ${ }^{2} \Pi_{u}$ state determined earlier, a Franck-Condon simulation similar to the one described above yields a bond length of $2.290 \pm 0.010 \AA$ for the $a^{1} \Delta_{g}$ state. This is the first time the $a^{1} \Delta_{g}$ state has been experimentally observed. The threshold spectrum also shows two peaks, I and i, separated by $547 \pm 10 \mathrm{~cm}^{-1}$. The remaining s-wave transition is the $b^{3} \Pi_{u} \leftarrow{ }^{2} \Sigma_{4}^{+}$transition, and these two peaks are assigned to the $0 \leftarrow 0$ and $1 \leftarrow 0$ vibrational transitions associated with this electronic transition. Using our bond length and vibrational frequency for the anion ${ }^{2} \Sigma_{u}^{+}$state, we determine $R_{e}=2.160 \pm 0.005 \AA$ for the $b^{\prime} \Pi_{u}$ state. This value agrees well with the experimental value of Davis $(2.16 \AA) ;^{19}$ he also found $v_{01}=540 \mathrm{~cm}^{-1}$ for the $b$ state, which agrees with our results. This supports our assignment of these peaks as well as our bond length estimate for the anion ${ }^{2} \Sigma_{\mathrm{g}}^{+}$state. Peaks $\mathrm{J}, \mathrm{K}$, and $\mathrm{L}$ appear in the photoelectron spectrum but not the threshold spectrum and are most likely due to p-wave transitions. Peaks I and $\mathrm{J}$ are separated by only $20 \mathrm{meV}$, which corresponds to the anion ${ }^{2} \Sigma_{g}^{+}-{ }^{2} \Pi_{u}$ splitting, so peak $J$ is assigned to the $0 \leftarrow 0$ peak of the $b^{1} \Pi_{u} \leftarrow-{ }^{2} \Pi_{u}$ transition. 
The transitions corresponding to peaks $I$ and $J$ involve removal of a $\pi_{u}$ and $\sigma_{g}$ electron, respectively, from the anion ${ }^{2} \Pi_{u}$ state and are therefore analogous to peaks $D$ and $d$ in the triplet region. In accord with this, we note that the $J / I$ and $D / d$ intensity ratios show similar dependence on the laser polarization angle in the photoelectron spectra.

We next consider transitions to the $c^{1} \Sigma_{8}{ }^{+}$and $\mathrm{d}^{1} \Sigma_{8}{ }^{+}$states of $\mathrm{Si}_{2}$. Davis ${ }^{19}$ found the $\mathrm{d}^{1} \Sigma_{\mathrm{g}}^{+}-\mathrm{b}^{1} \Pi_{\mathrm{u}}$ splitting to be $4900 \mathrm{~cm}^{-1}(0.608 \mathrm{eV})$, so transitions to the $\mathrm{d}^{1} \Sigma_{\mathrm{g}}^{+}$state would not appear in the $355 \mathrm{~nm}$ photoelectron spectrum (the TOF analyzer transmission is poor for eKE $<0.3 \mathrm{eV}$ ). Either peak $\mathrm{K}$ or $\mathrm{L}$ could be from a transition to the $\mathrm{c}^{1} \Sigma_{\mathrm{B}}^{+}$state, however. The question of whether the dominant $\mathrm{MO}$ configuration in the $\mathrm{c}^{\prime} \Sigma_{\mathrm{g}}^{+}$state is (a) $\pi_{u}{ }^{4}$ or (b) $\sigma_{\mathrm{g}}{ }^{2} \pi_{\mathrm{u}}{ }^{2}$ has been raised in the ab initio studies of Bruna ${ }^{11}$ and McLean. ${ }^{12}$ If (b) were the case, then the $c^{1} \Sigma_{g}^{+}$state would be accessible by a one-electron transition only from the anion ${ }^{2} \Pi_{u}$ state, and this can go by an s-wave. On the other hand, if $\pi_{u}{ }^{4}$ were the dominant configuration, this state could be reached by a one-electron transition from the anion ${ }^{2} \Sigma_{B}^{+}$state by p-wave detachment. Peaks $K$ and $L$ are both absent in the the threshold spectrum, which indicates they are both from p-wave detachment transitions. Assuming either $K$ or $L$ is a transition to the $c^{1} \Sigma_{g}{ }^{+}$state, this implies that $\pi_{u}{ }^{4}$ is the dominant $\mathrm{MO}$ configuration of the $\mathrm{c}^{1} \Sigma_{\mathrm{B}}^{+}$state, and that $\mathrm{K}$ or $\mathrm{L}$ is the $\mathrm{c}^{1} \Sigma_{\mathrm{B}}^{+} \leftarrow{ }^{2} \Sigma_{\mathrm{B}}^{+}$ transition.

We note that peak $F$ in the triplet band was not assigned to any of the transitions in Figure 7, and that it has the same dependence on laser polarization angle as peak $\mathrm{L}$. This suggests that peaks $F$ and $L$ are due to transitions to different spin multiplets of the same $\mathrm{Si}_{2}$ electronic state ( $F$ to the triplet, $\mathrm{L}$ to the singlet) and that peak $\mathrm{K}$ is the $\mathrm{c}^{1} \Sigma_{\mathrm{g}}{ }^{+} \leftarrow$ 
${ }^{2} \Sigma_{g}+$ transition. This assignment is speculative, however, in that it is not clear what neutral $\leftarrow$ anion transition leads to peaks $F$ and $L$.

We conclude by comparing the silicon dimer to the isovalent $\mathrm{C}_{2}$ and $\mathrm{SiC}$ molecules. Just as in the case of the silicon dimer, a long standing controversy about the low-lying electronic states of $\mathrm{C}_{2}$ was finally settled by Ballik and Ramsay, ${ }^{41}$ establishing the ${ }^{1} \Sigma_{g}{ }^{+}\left(\sigma_{g}{ }^{0} \pi_{u}{ }^{4}\right)$ as the ground state. The ${ }^{3} \Pi_{u}$ and ${ }^{3} \Sigma_{g}$ states were found to lie $0.089 \mathrm{eV}$ and $0.798 \mathrm{eV}$ above the ground state respectively. It appears that $C_{2}$ is small enough to allow the $\pi$ orbitals to interact very strongly leading to a ground state which maximizes $\pi$-bonding. In the case of the $\mathrm{SiC}$ radical, Brazier et. al. ${ }^{42}$ report a $0.558 \mathrm{eV}$ splitting between the $X^{3} \Pi_{u}\left(\sigma_{g}{ }^{1} \pi_{u}{ }^{3}\right)$ ground state and the $A^{3} \Sigma_{g} \cdot\left(\sigma_{g}{ }^{2} \pi_{u}{ }^{2}\right)$ first excited state, while the ${ }^{1} \Sigma_{B}^{+}$was found to be higher in energy than both of the triplet states. Thus, in SiC, for which the bond length is greater than $\mathrm{C}_{2}$, the $\pi$ orbital interaction has weakened while the $\sigma$ orbitals are becoming important in stabilizing the molecule. Extrapolating from this trend, one expects $\sigma$ bonding to have an even greater effect in the silicon dimer. Hence we expect the ${ }^{3} \Sigma_{\mathrm{g}}$ state to be the ground state, which agrees with the experimental results. The correlation of longer bond length with more $\sigma$ bonding holds for all the $\mathrm{Si}_{2}$ and $\mathrm{Si}_{2}$. electronic states; the $\sigma^{2}$ states $\left({ }^{2} \Pi_{u}, X^{3} \Sigma_{g}, a^{1} \Delta_{g}\right)$ have longer bond lengths than the $\sigma^{1}$ states $\left({ }^{2} \Pi_{u}, D^{3} \Pi_{u}, b^{1} \Pi_{u}\right)$.

\section{Conclusion}

Using negative ion photoelectron spectroscopy and threshold photodetachment, we have sorted out the low-lying electronic states in $\mathrm{Si}_{2}$ and $\mathrm{Si}_{2}{ }^{-}$. The complexity of the electronic structure of this diatom indicates that the spectroscopy of larger Si clusters may 
be quite challenging. Nonetheless, the combination of the two techniques used in this work presents a promising approach to this problem. We have already obtained vibrationally resolved photoelectron spectra of $\mathrm{Si}_{3}{ }^{-}$and $\mathrm{Si}_{4}{ }^{-43}$ and of several carbon cluster anions ${ }^{44}$, as well as the threshold photodetachment spectra of $\mathrm{C}_{5}{ }^{-}$which will be presented in the chapters that follow. 


\section{Figure Captions}

1. The six low-lying states resulting from the three possible valence orbital configurations of $\mathrm{Si}_{2}$.

2. Detection schemes for the fixed frequency photoelectron spectrometer and the threshold photodetachment spectrometer.

3. Photoelectron spectra of $\mathrm{Si}_{2}{ }^{-}$at $355 \mathrm{~nm}(3.49 \mathrm{eV})$, with the laser polarization parallel to the direction of electron detection.

4. Photoelectron spectra of $\mathrm{Si}_{2}-$ at $466 \mathrm{~nm}(4.66 \mathrm{eV})$, with the direction of the laser polarization perpendicular to the direction of electron detection.

5. Threshold photodetachment spectrum of $\mathrm{Si}_{2}{ }^{-}$showing the $\mathrm{X}^{3} \Sigma_{\mathrm{g}} \leftarrow{ }^{2} \Pi_{u}$ and the $D^{3} \Pi_{u} \leftarrow{ }^{2} \Sigma_{8}^{+}$neutral $\leftarrow$ anion electronic transitions. In the simulated spectrum shown, the peak widths were set at $0.005 \mathrm{eV}\left(40 \mathrm{~cm}^{-1}\right)$.

6. The threshold photodetachment spectrum of $\mathrm{Si}_{2}-$ showing the $\mathrm{a}^{\mathrm{d}} \Delta_{\mathrm{B}} \leftarrow^{2} \Pi_{u}$ and the $b^{\prime} \Pi_{u} \leftarrow{ }^{2} \Sigma_{g}^{+}$neutral $\leftarrow$ anion transitions.

7. Energy level diagram showing the one-electron transitions between the ${ }^{2} \Pi_{u}$ and ${ }^{2} \Sigma_{B}^{+}$ electronic states of $\mathrm{Si}_{2}{ }^{-}$and the triplet electronic states of $\mathrm{Si}_{2}$.

8. Energy level diagram showing the electronic transitions between the ${ }^{2} \Pi_{u}$ and ${ }^{2} \Sigma_{g}^{+}$ electronic states of $\mathrm{Si}_{2}{ }^{-}$and the singlet electronic states of $\mathrm{Si}_{2}$. Peaks in the spectra that correspond to each transition are shown in parentheses. The transition to the $\mathrm{d}^{1} \Sigma_{\mathrm{g}}{ }^{+}$state was not observed. 


\section{Table Captions}

1. Peak positions and assignments for the photoelectron and threshold photodetachment spectra of $\mathrm{Si}_{2}-$. Peaks appearing only in the photoelectron spectra are marked with an asterisk $\left(^{*}\right)$ and their energy uncertainty is $\pm 0.010 \mathrm{eV}$. The energy uncertainty of al! other peaks in the table is $\pm 0.001 \mathrm{eV}$.

2. Spectroscopic constants for the first two electronic states of $\mathrm{Si}_{2}-$.

3. Spectroscopic constants for the first five electronic states of $\mathrm{Si}_{2}$. 
Table 1:

\begin{tabular}{|c|c|c|}
\hline Peak & Position (eV) & $\mathbf{S i}_{2} \leftarrow \mathbf{S i}_{2}^{-}$ \\
\hline$A^{*}$ & 2.052 & ${ }^{3} \Sigma_{g} \cdot\left(v^{\prime}=0\right) \leftarrow{ }^{2} \Pi_{u}\left(v^{\prime \prime}=2\right)$ \\
\hline $\mathrm{B}_{2}$ & 2.095 & ${ }^{3} \Sigma_{\mathbf{g}}\left(v^{\prime}=0\right) \leftarrow{ }^{2} \Pi_{1 / 2}\left(v^{\prime \prime}=1\right)$ \\
\hline $\mathrm{B}_{1}$ & 2.109 & ${ }^{3} \Sigma_{8}\left(v^{\prime}=0\right) \leftarrow{ }^{2} \Pi_{3 / 2}\left(v^{\prime \prime}=1\right)$ \\
\hline $\mathrm{C}_{2}$ & 2.161 & ${ }^{3} \Sigma_{\mathbf{g}}\left(v^{\prime}=0\right) \leftarrow{ }^{2} \Pi_{1 / 2}\left(v^{\prime \prime}=0\right)$ \\
\hline$c_{1}^{\prime}$ & 2.169 & ${ }^{3} \Pi_{2}\left(v^{\prime}=0\right) \leftarrow{ }^{2} \Sigma_{g}^{+}\left(v^{\prime \prime}=1\right)$ \\
\hline $\mathrm{C}_{1}$ & 2.176 & ${ }^{3} \Sigma_{\mathbf{g}}\left(v^{\prime}=0\right) \leftarrow{ }^{2} \Pi_{3 / 2}\left(v^{\prime \prime}=0\right)$ \\
\hline$c_{3}^{\prime}$ & 2.185 & ${ }^{3} \Pi_{0}\left(v^{\prime}=0\right) \leftarrow{ }^{2} \Sigma_{g}^{+}\left(v^{\prime \prime}=1\right)$ \\
\hline$c_{2}$ & 2.225 & ${ }^{3} \Sigma_{\mathbf{B}}\left(v^{\prime}=1\right) \leftarrow{ }^{2} \Pi_{1 / 2}\left(v^{\prime \prime}=0\right)$ \\
\hline$d_{1}$ & 2.235 & ${ }^{3} \Pi_{2}\left(v^{\prime}=0\right) \leftarrow{ }^{2} \Sigma_{g}^{+}\left(v^{\prime \prime}=0\right)$ \\
\hline$d_{2}$ & 2.242 & ${ }^{3} \Pi_{1}\left(v^{\prime}=0\right) \leftarrow{ }^{2} \Sigma_{g}^{+}\left(v^{\prime \prime}=0\right)$ \\
\hline$d_{3}$ & 2.250 & ${ }^{3} \Pi_{0}\left(v^{\prime}=0\right) \leftarrow{ }^{2} \Sigma_{g}^{+}\left(v^{\prime \prime}=0\right)$ \\
\hline$D^{*}$ & 2.259 & ${ }^{3} \Pi_{0,1,2}\left(v^{\prime}=0\right) \leftarrow{ }^{2} \Pi_{1 / 2,3 / 2}\left(v^{\prime \prime}=0\right)$ \\
\hline$e_{1}$ & 2.301 & ${ }^{3} \Pi_{2}\left(v^{\prime}=1\right) \leftarrow{ }^{2} \Sigma_{B}^{+}\left(v^{\prime \prime}=0\right)$ \\
\hline$e_{2}$ & 2.309 & ${ }^{3} \Pi_{1}\left(v^{\prime}=1\right) \leftarrow{ }^{2} \Sigma_{\mathrm{g}}^{+}\left(v^{\prime \prime}=0\right)$ \\
\hline$e_{3}$ & 2.316 & ${ }^{3} \Pi_{0}\left(v^{\prime}=1\right) \leftarrow{ }^{2} \Sigma_{g}^{+}\left(v^{\prime \prime}=0\right)$ \\
\hline
\end{tabular}


Table 1 (continued):

\begin{tabular}{|c|c|c|}
\hline Peak & Position (eV) & $\mathrm{Si}_{2} \leftarrow \mathrm{Si}_{2}^{-}$ \\
\hline$E^{*}$ & 2.323 & ${ }^{3} \Pi_{0,1,2}\left(v^{\prime}=1\right) \leftarrow{ }^{2} \Pi_{1 / 2,3 / 2}\left(v^{\prime \prime}=0\right)$ \\
\hline$F^{*}$ & 2.335 & $?$ \\
\hline $\mathrm{G}_{1}$ & 2.598 & ${ }^{1} \Delta_{8}\left(v^{\prime}=0\right) \leftarrow{ }^{2} \Pi_{1 / 2}\left(v^{\prime \prime}=0\right)$ \\
\hline $\mathrm{G}_{2}$ & 2.611 & ${ }^{1} \Delta_{g}\left(v^{\prime}=0\right) \leftarrow{ }^{2} \Pi_{3 / 2}\left(v^{\prime \prime}=0\right)$ \\
\hline $\mathrm{H}_{1}$ & 2.658 & ${ }^{1} \Delta_{B}\left(v^{\prime}=1\right) \leftarrow{ }^{2} \Pi_{1 / 2}\left(v^{\prime \prime}=0\right)$ \\
\hline $\mathrm{H}_{2}$ & 2.672 & ${ }^{1} \Delta_{g}\left(v^{\prime}=1\right) \leftarrow{ }^{2} \Pi_{3 / 2}\left(v^{\prime \prime}=0\right)$ \\
\hline $\mathrm{M}_{1}$ & 2.715 & ${ }^{1} \Delta_{g}\left(v^{\prime}=2\right) \leftarrow{ }^{2} \Pi_{1 / 2}\left(v^{\prime \prime}=0\right)$ \\
\hline $\mathrm{M}_{2}$ & 2.724 & ${ }^{1} \Delta_{B}\left(v^{\prime}=2\right) \leftarrow{ }^{2} \Pi_{3 / 2}\left(v^{\prime \prime}=0\right)$ \\
\hline I & 2.745 & ${ }^{1} \Pi_{u}\left(v^{\prime}=0\right) \leftarrow{ }^{2} \Sigma_{B}^{+}\left(v^{\prime \prime}=0\right)$ \\
\hline $\mathrm{i}$ & 2.812 & ${ }^{1} \Pi_{u}\left(v^{\prime}=1\right) \leftarrow{ }^{2} \Sigma_{g}^{+}\left(v^{\prime \prime}=0\right)$ \\
\hline $\mathrm{J}^{*}$ & 2.753 & ${ }^{1} \Pi_{u}\left(v^{\prime}=0\right) \leftarrow{ }^{2} \Pi_{1 / 2.3 / 2}\left(v^{\prime \prime}=0\right)$ \\
\hline $\mathrm{K}^{*}$ & 2.789 & ${ }^{1} \Sigma_{g}^{+}\left(v^{\prime}=0\right) \leftarrow{ }^{2} \Sigma_{g}^{+}\left(v^{\prime \prime}=0\right)$ \\
\hline $\mathrm{L}^{\circ}$ & 2.825 & $?$ \\
\hline
\end{tabular}


Table 2:

\begin{tabular}{|c|c|c|c|c|}
\hline $\mathrm{Si}_{2}-$ State & $v\left(\mathrm{~cm}^{-1}\right)$ & $\mathrm{r}_{\mathrm{e}}(\AA)$ & $\mathrm{A}\left(\mathrm{cm}^{-1}\right)$ & $\mathrm{T}_{\mathrm{e}}(\mathrm{eV})$ \\
\hline${ }^{2} \Sigma_{\mathrm{g}}^{+}$ & $528 \pm 10$ & $2.116 \pm .005$ & - & $.025 \pm .010$ \\
\hline${ }^{2} \Pi_{u}$ & $533 \pm 5$ & $2.207 \pm .005$ & $-122 \pm 5$ & 0 \\
\hline
\end{tabular}

Table 3:

\begin{tabular}{|c|c|c|c|c|}
\hline $\mathrm{Si}_{2}$ State & $v\left(\mathrm{~cm}^{-1}\right)$ & $\mathrm{r}_{\mathrm{e}}(\AA)$ & $\mathrm{A}\left(\mathrm{cm}^{-1}\right)$ & $\mathrm{T}_{\mathrm{c}}(\mathrm{eV})$ \\
\hline $\mathrm{X}^{3} \Sigma_{\mathrm{B}}$ & $509 \pm 10$ & 2.246 & - & 0 \\
\hline $\mathrm{D}^{3} \Pi_{u}$ & $536 \pm 5$ & 2.115 & $-64 \pm 10$ & $.083 \pm .010$ \\
\hline $\mathrm{a}^{1} \Delta_{\mathrm{g}}$ & $486 \pm 10$ & $2.290 \pm .010$ & - & $.435 \pm .001$ \\
\hline $\mathrm{b}^{1} \Pi_{\mathrm{u}}$ & $540 \pm 10$ & $2.160 \pm .005$ & - & $.593 \pm .010$ \\
\hline $\mathrm{c}^{1} \Sigma_{\mathrm{g}}^{+}$ & $365^{*}$ & $2.16-2.31^{*}$ & - & $.637 \pm .010$ \\
\hline
\end{tabular}

* Ref. 18,19. 


\section{$\mathrm{Si}_{2}$}
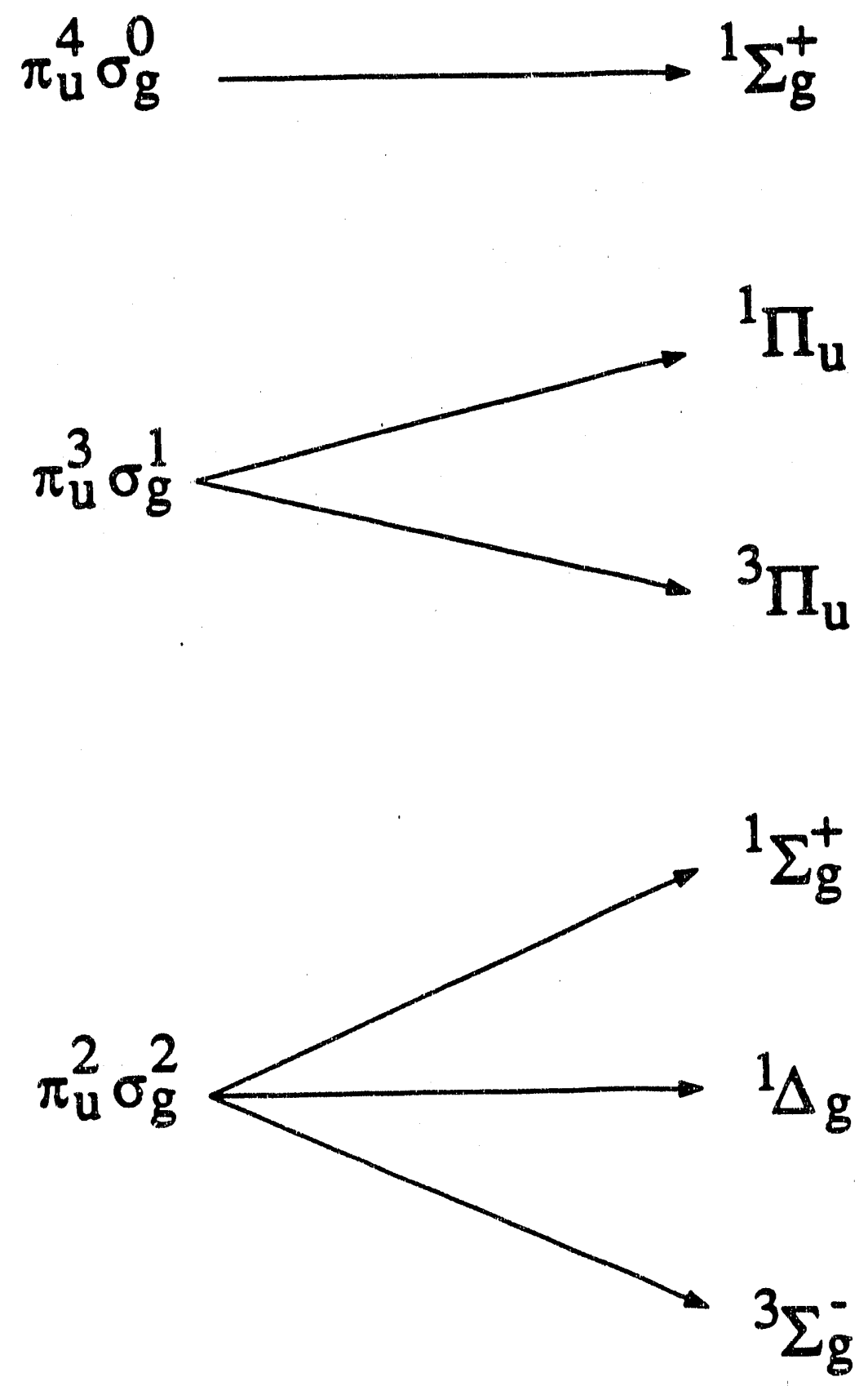

Figure 1 
Fixed Frequency Photoelectron Spectroscopy

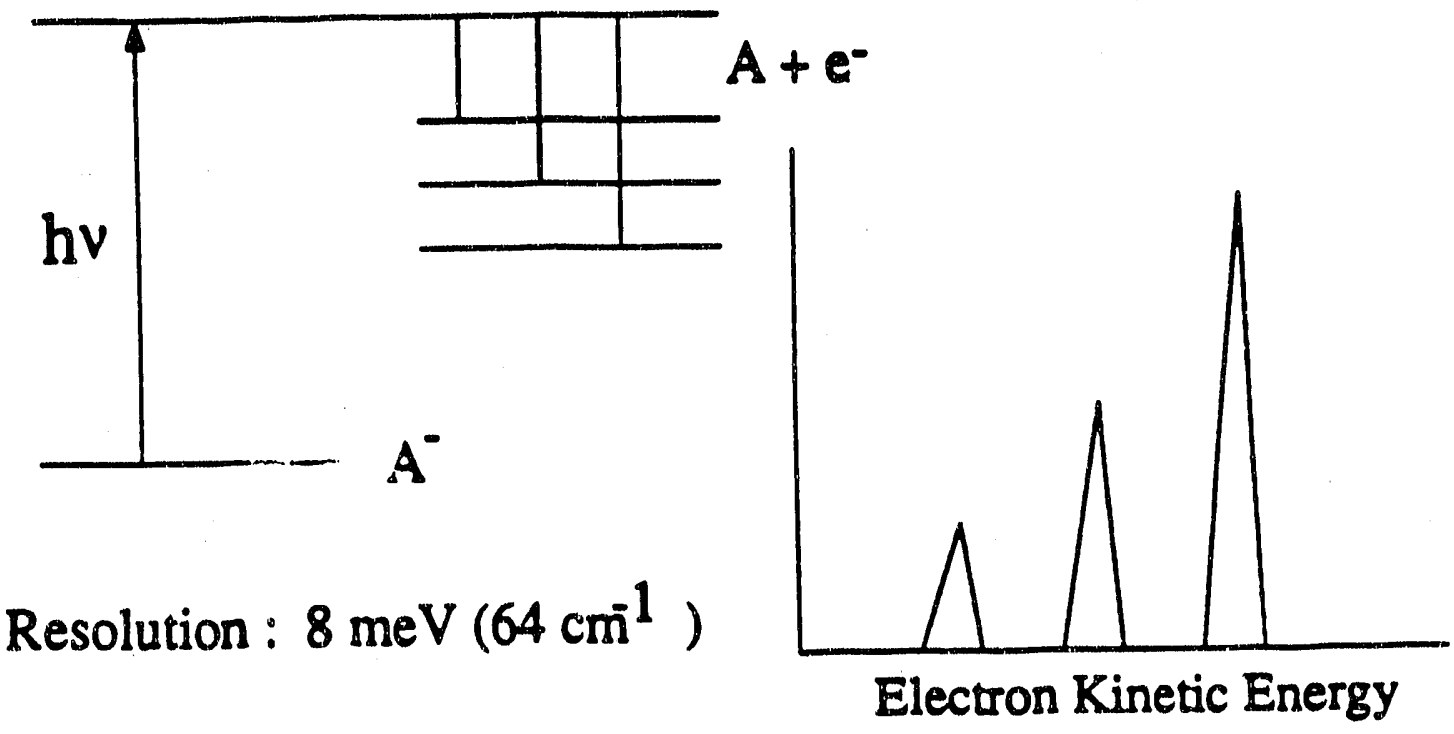

Threshold Photodetachment Spectroscopy
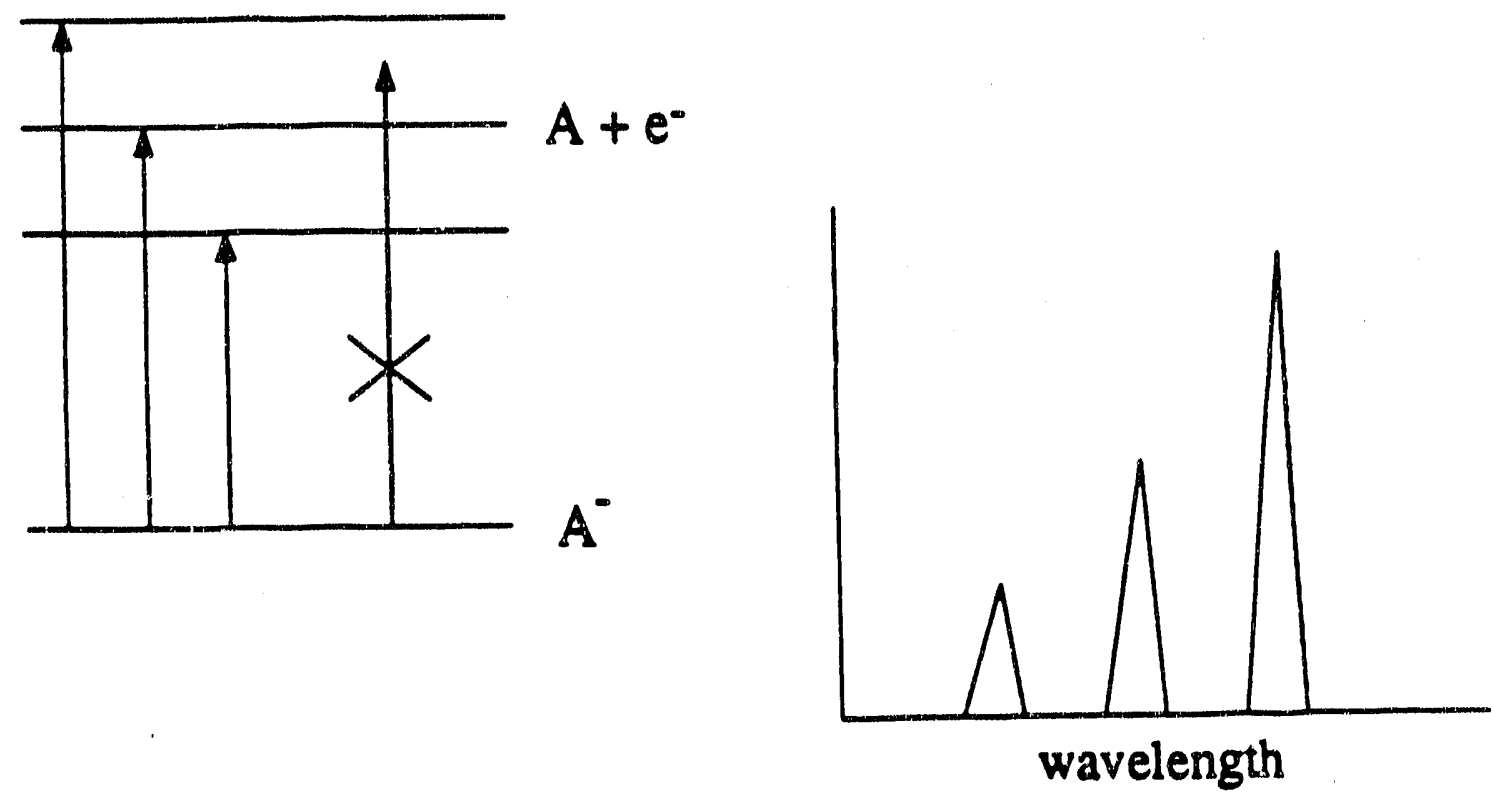

Resolution : $0.38 \mathrm{meV}\left(3 \mathrm{~cm}^{-1}\right)$

Figure 2 


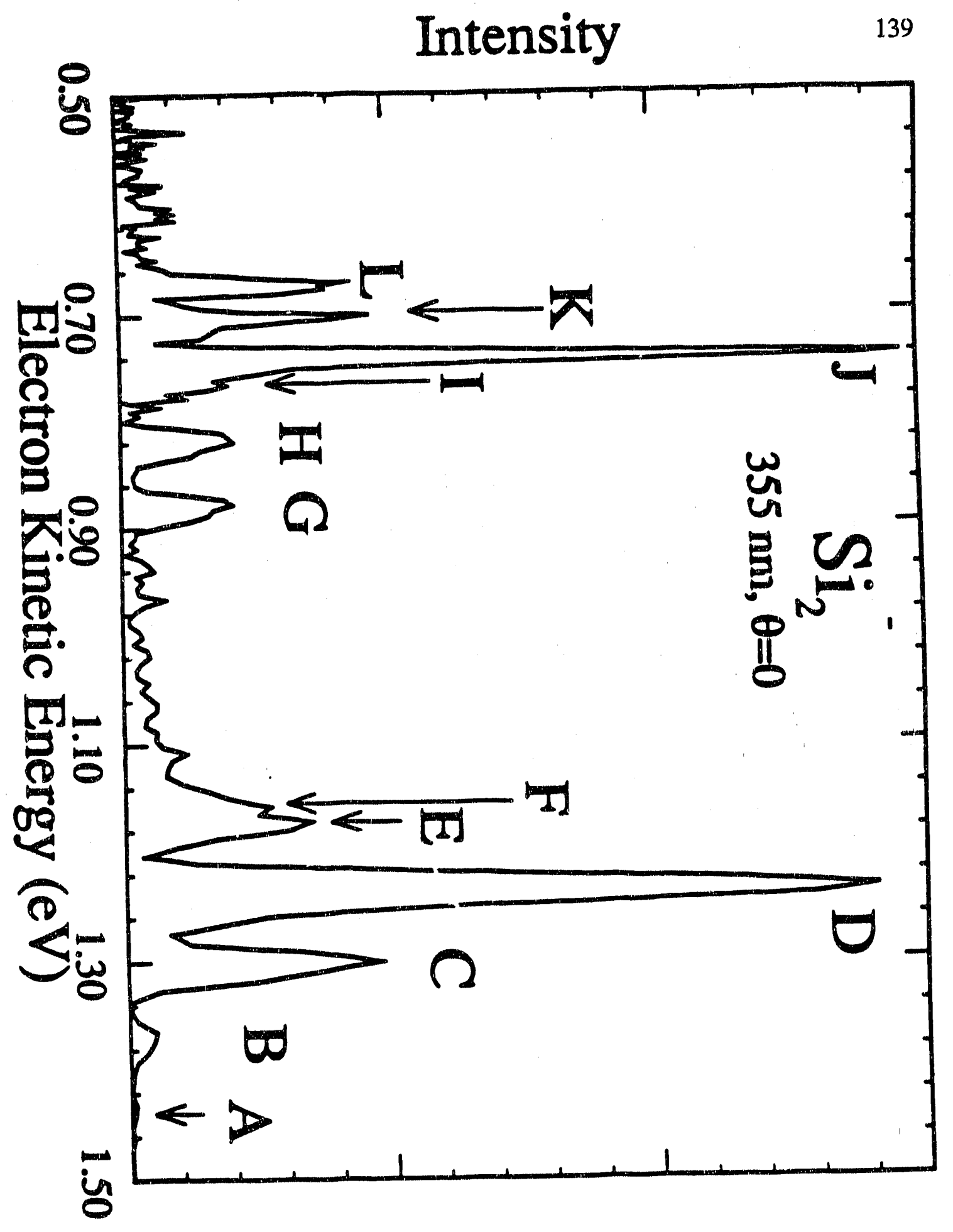

Figure 3 


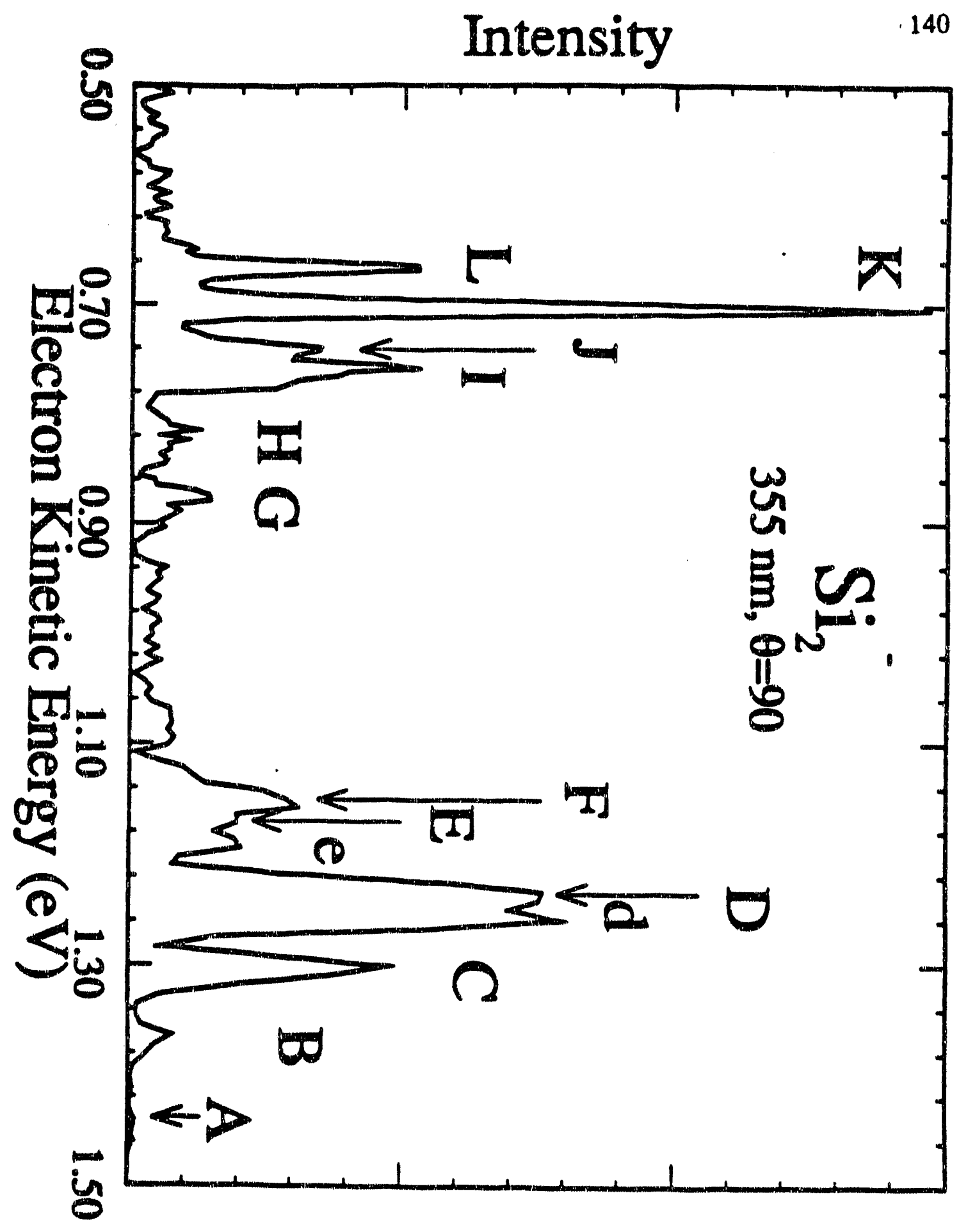

Figure 4 


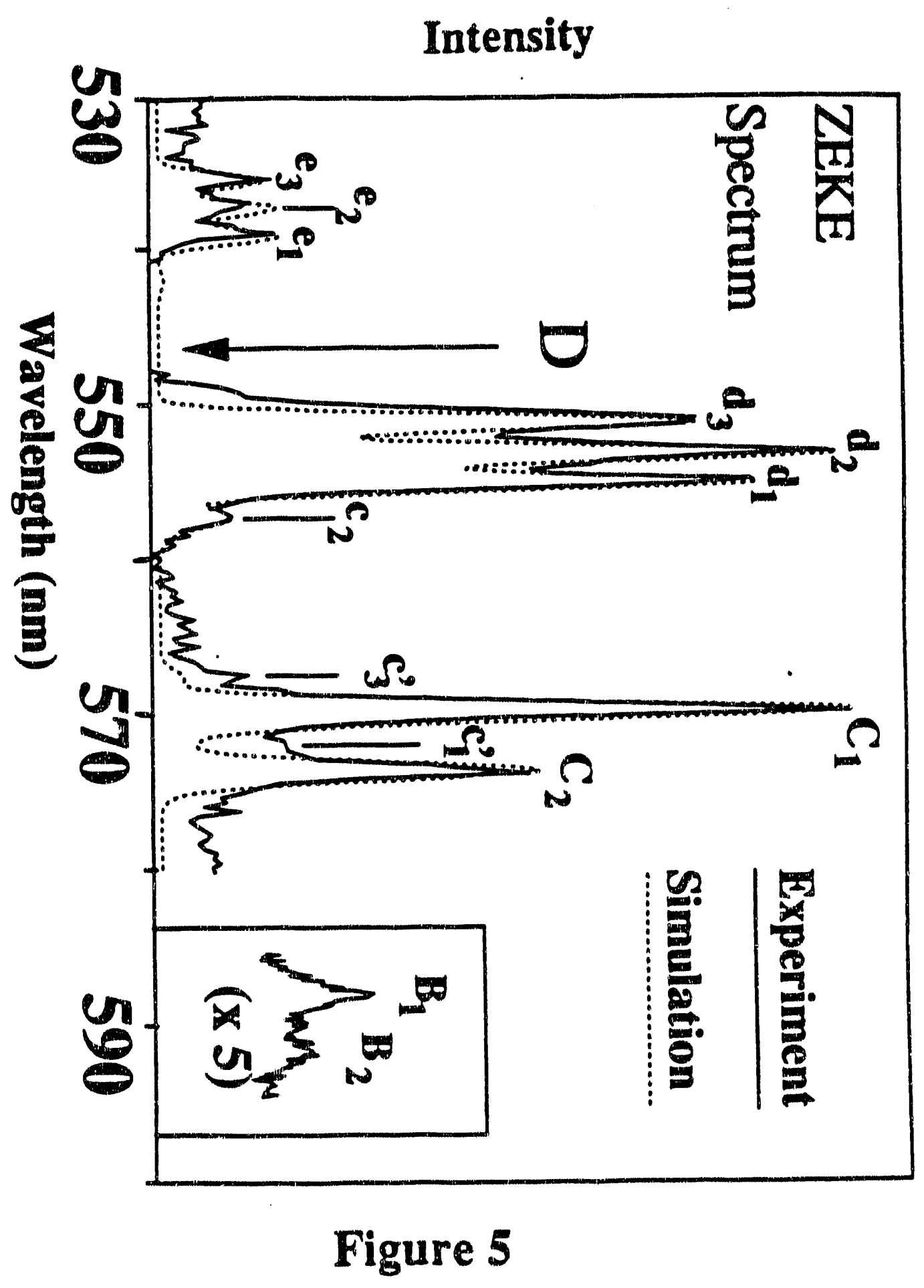




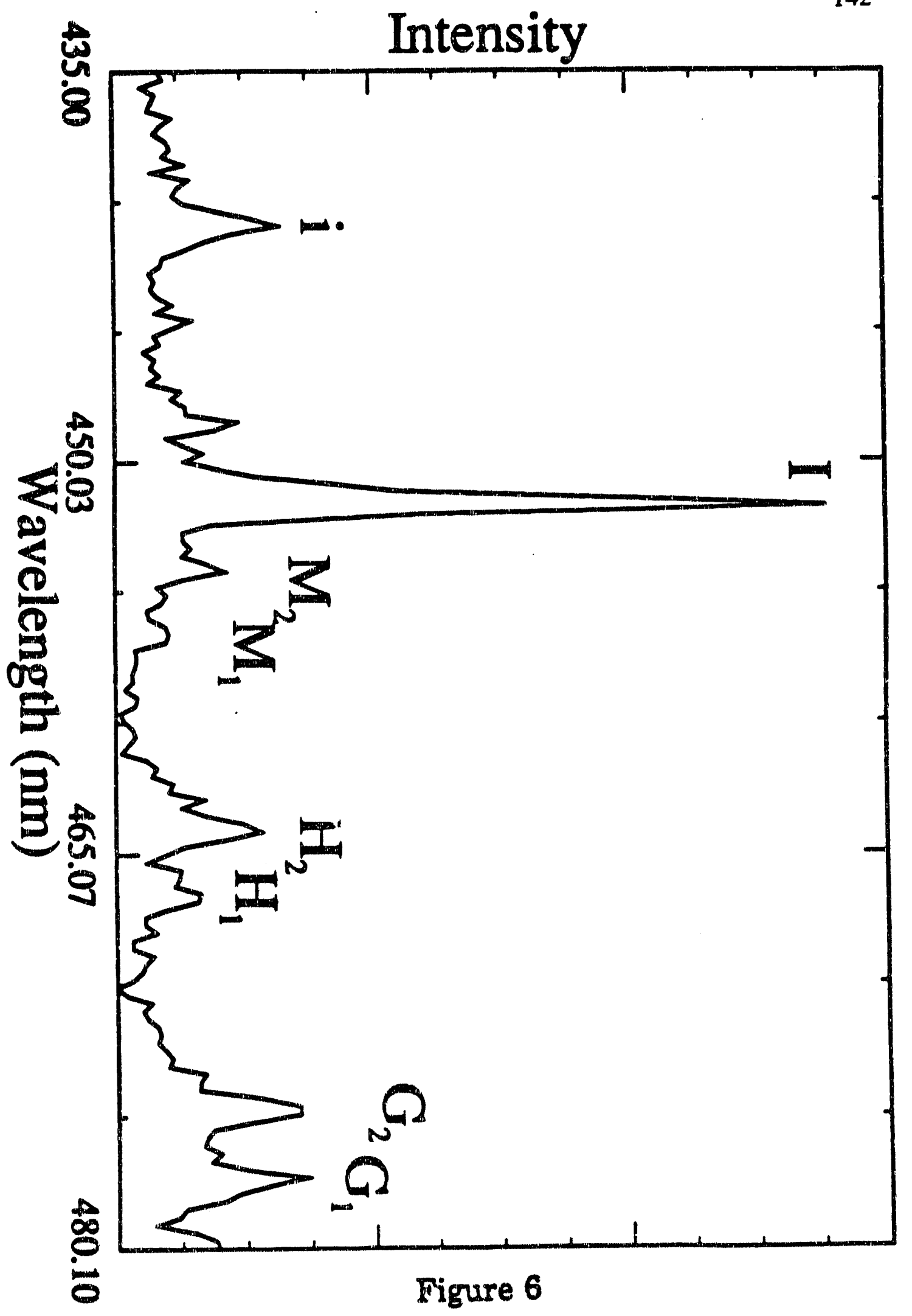




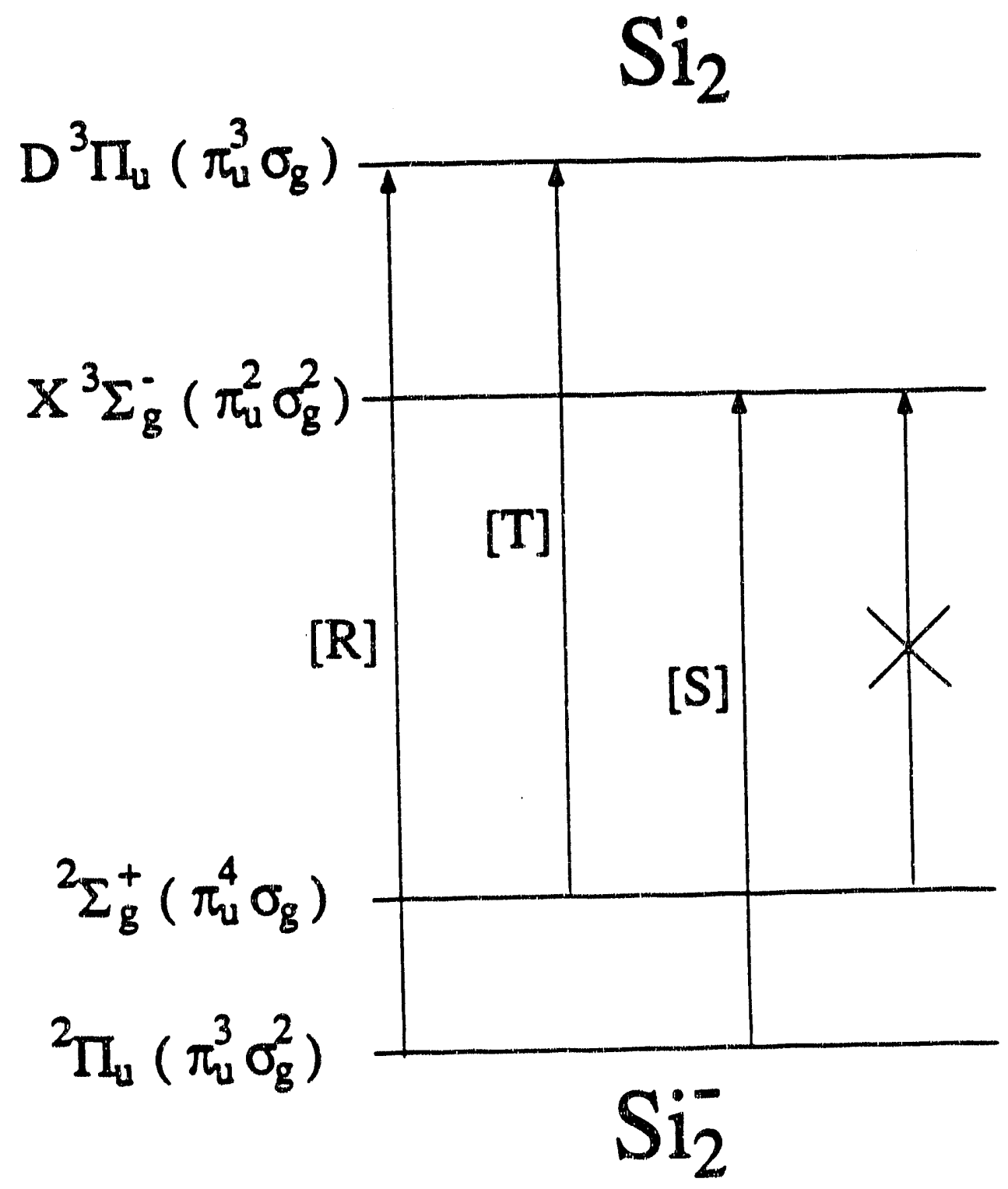

Figure 7 
$\mathrm{d}^{1} \Sigma_{\mathrm{g}}^{+}$

$\mathrm{c}^{1} \Sigma_{\mathrm{g}}^{+}$

$\mathrm{b}^{1} \Pi_{\mathrm{u}}$

${ }^{1}{ }^{1} \Delta_{g}$

$D^{3} \Pi_{u}$

$\mathrm{X}^{3} \Sigma_{\mathrm{g}}^{-}$
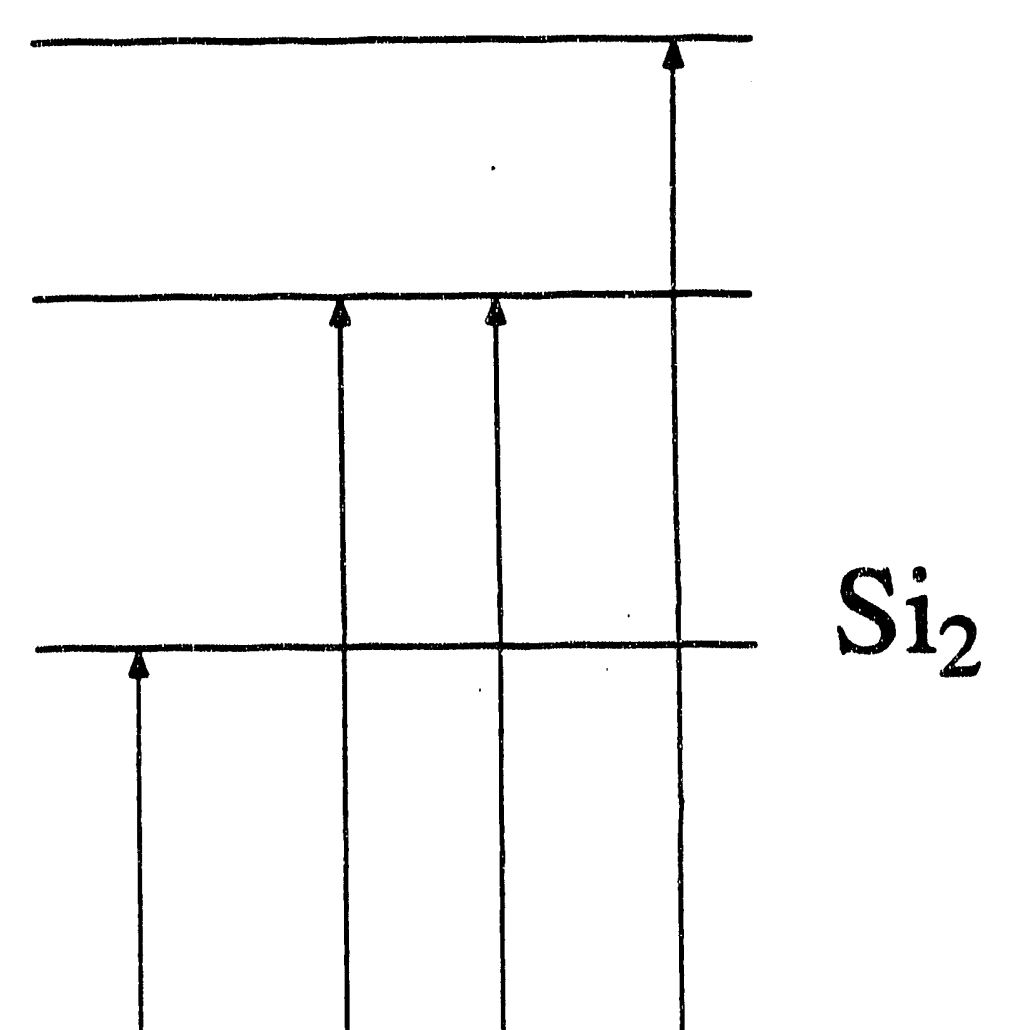

${ }^{2} \Sigma_{\mathrm{g}}^{+}$

${ }^{2} \Pi_{u}$

$\mathrm{Si}_{2}^{-}$

Figure 8 


\section{References}

1. Metal Clusters, edited by M. Moskovits (Wiley, New York, 1986).

2. M.D. Morse, Chem. Rev. 86, 1049 (1986).

3. Physics and Chemistry of Small Clusters, edited by P. Jena, B. K. Rao, and S. N. Kanna, NATO Advanced Science Institutes Series 158 (Plenum Press, New York, 1987).

4. W. Weltner and R.J. Van Zee, Chem. Rev. 89, 1713 (1989).

5. K. Balasubramanian, Chem. Phys. Lett. 125, 400 (1986).

6. K. Raghavachari, J. Chern. Phys. 83, 3520 (1985).

7. G. Pacchioni and J. Koutecky, J. Chem. Phys. 84, 3301, 1986; V. Bonacic-Koutecky,

P. Fantucci, and J. Koutecky, J. Chem. Phys. 93, 3802 (1990).

8. H. Basch, Chem. Phys. Lett. 136, 289 (1987).

9. C.W. Bauschlicher, Jr., and S.R. Langhoff, J. Chem. Phys. 87, 2919 (1987).

10. K. Raghavachari, J. Chem. Phys. 84, 5672 (1986).

11. P.J. Bruna, S.D. Peyerimhoff, and R.J. Buenker, J. Chem. Phys. 72, 5437 (1980).

12. A.D. McLean, B. Liu, and G.S. Chandler, J. Chem. Phys. 80, 5130 (1984).

13. H.P. Lüthi and A.D. McLean, Chem. Phys. Lett. 135, 352 (1987).

14. F. Müller-Plathe and L. Laaksonen, Chem. Phys. Lett. 160, 175 (1989).

15. A.E. Douglas, Can. J. Phys. 33, 801 (1955).

16. I. Dubois, and H. Leclercq, Can. J. Phys. 49, 3053 (1971); D.E. Milligan, and M.E. Jacox, J. Can. Phys. 52, 2594(1969); R.D. Verma, and P.A. Warsop, Can. J. Phys. 41, 152 (1963); A. Lagerquist, and C. Malmberg, Physica Scripta 2, 45 (1970).

17. M.R. Nimlos, L.B. Harding, and G.B. Ellison, J. Chem. Phys. 87, 5116 (1987).

18. I. Dubois and H. Leclerca, J. Phys. B 14, 2807 (1981); Can. J. Phys. 49, 3053 (1971). 
19. S.P. Davis and J.W. Brault, J. Opt. Soc. Am. B 4, 20 (1987).

20. P.J. Bruna, H. Dohmann, J. Anglada, V. Krumbach, S.D. Peyerimhoff, and R.J. Buenker, J. Mol. Struct. 93, 309 (1983).

21. K. Ragavachari and C.M. Rohlfing, J. Chem. Phys. 94, 3670 (1991).

22. J. Ho, K.M. Ervin, and W.C. Lineberger, J. Chem. Phys. 93, 6987 (1990).

23. O. Cheshnovsky, S.H. Yang, C.L. Pettiette, M.J. Craycraft, Y. Liu, and R.E. Smalley, Chem. Phys. Lett. 138, 119 (1987).

24. L. A. Posey, M. J. DeLuca, and M. A. Johnson, Chem. Phys. Lett. 131, 170 (1986);

L. A. Posey and M. A. Johnson, J. Chem. Phys. 88, 5383 (1988).

25. K. M. McHugh, J. G. Eaton, G. H. Lee, H. W. Sarkas, L. H. Kidder, J. T. Snodgrass, M. R. Manaa, and K. H. Bowen, J. Chem. Phys. 91, 3792 (1989).

26. G. Ganteför, M. Gausa, K.H. Meiwes-Broer, and H.O. Lutz, Z. Phys. D 9, 253 (1988). 27. D.G. Leopold, K.K. Murray, A.E. Stevens Miller, and W.C. Lineberger, J. Chem. Phys. 83, 4849 (1985).

28. R.B. Metz, A. Weaver, S.E. Bradforth, T.N. Kitsopoulos, and D.M. Neumark, J. Phys. Chem. 94, 1377 (1990).

29. T.N. Kitsopoulos, I.M. Waller, J.G. Loeser, and D.M. Neumark, Chem. Phys. Lett. 159, 300 (1989).

30. T.G. Dietz, M.A. Duncan, D.E. Powers, and R.E. Smalley, J. Chem. Phys. 74, 6511 (1981); V.E. Bondybey, and J.H. English J. Chem. Phys. 74, 6978 (1981).

31. W.C. Wiley and I.H. McLaren, Rev. Sci. Instr. 26, 1150 (1955).

32. J.M.B. Bakker, J. Phys. E 6, 785 (1972); 7, 364 (1974).

33. K. Müller-Dethlefs, M. Sander, and E.W. Schlag, Z. Naturforsch. 39a, 1089 (1984); 
Chem. Phys. Lett. 12, 291 (1984); M. Sander, L.A. Chewter, K. Müller-Dethlefs, and E.W. Schlag, Phys. Rev. A 36, 4543 (1987).

34. G.F. Gantefor, D.M. Cox, and A. Kaldor, J. Chem. Phys. 94, 854 (1990).

35. A preliminary analysis of the photoelectron spectra was presented in the MRS Proceedings, Fall 1990 Meeting.

36. D.G. Leopold and W.C. Lineberger, J. Chem. Phys. 85, 51 (1986).

37. J. Cooper and R.N. Zare, J. Chem. Phys. 48, 942 (1968).

38. K.P. Huber, and G. Herzberg, Molecular Spectra and Molecular Structure IV, Constants of Diatomic Molecules, (Van Nostrand Reinhold, New York, 1979).

39. E.P. Wigner, Phys. Rev. 73, 1003 (1948).

40. K.J. Reed, A.H. Zimmerman, H.C. Andersen, and J.I. Brauman, J. Chem. Phys. 64, 1368 (1976).

41. E.A. Ballik and D.A. Ramsay, Astrophys. J. 137, 84 (1963); and also Astrophys. J. 137, 61 (1963).

42. C.R. Brazier, L.C. O'Brien, and P.F. Bernath, J. Chem. Phys. 91, 7384 (1989).

43. T.N. Kitsopoulos, C.J. Chick, A. Weaver, and D.M. Neumark, J. Chem. Phys. 93, 6108 (1990).

44. D. W. Arnold, T. N. Kitsopoulos, and D. M. Neumark, to be published. 


\section{Chapter V}

\section{Vibrationally resolved photoelectron spectra of $\mathrm{Si}_{3}{ }^{-}$and $\mathrm{Si}_{4}{ }^{-}$}

\section{Introduction}

The study of elemental clusters has received a great deal of experimental attention in recent years. A major goal of this effort is to understand how the structure and properties of a cluster depend on its size. However, little is known about the spectroscopy of clusters with three or more atoms. For example, spectra showing resolved vibrational features have been observed for only a handful of such clusters in the gas phase. ${ }^{1.23,45,6.7}$ The measurement of vibrational frequencies is an important first step towards understanding the nature of chemical bonding in a cluster.

Negative ion photoelectron spectroscopy offers a promising experimental technique for the study of size-selected elemental clusters. In a typical experiment, a mass-selected beam of negative cluster ions is photodetached and the resulting electron kinetic energy distribution is analyzed, thereby probing the electronic and vibrational states of the neutral cluster formed by photodetachment. This technique offers the considerable advantage of eliminating any ambiguity concerning the size of the cluster under investigation, and has been used by Lineberger, ${ }^{5,8}$ Smalley, ${ }^{9,10,11}$ Bowen, ${ }^{12}$ Meiwes-Bröer, ${ }^{13}$ and their coworkers to study polyatomic metal and semiconductor clusters. However, the resolution of a photoelectron spectrometer is, at best, $50-100 \mathrm{~cm}^{-1}$, and only Lineberger's spectra ${ }^{5}$ of $\mathrm{Pd}_{3}-$ and $\mathrm{Pt}_{3}-$ have shown resolved vibrational structure.

In this chapter we present photoelectron spectra of $\mathrm{Si}_{3}{ }^{-}$and $\mathrm{Si}_{4}{ }^{-}$which show resolved vibrational progressions in several electronic states of the neutral clusters. The 
photoelectron spectra of these anions were first reported by Smalley, ${ }^{9}$ but the resolution of his spectra was around $1200 \mathrm{~cm}^{-1}$. Our spectra are obtained at considerably higher resolution, thereby enabling the observation of vibrational structure. The interpretation of these spectra is aided by the ab initio calculations on small neutral Si clusters which have been performed by several investigators, ${ }^{14,15,16,17,18,19,20,21}$ and by recent calculations on Si cluster anions by Raghavachari and Rohlfing. ${ }^{22}$

\section{Experimental}

The instrument used in these studies is a modified version of our negative ion time-of-flight photoelectron spectrometer described in detail elsewhere. ${ }^{23}$ The instrument is similar to that first described by Johnson and co-workers. ${ }^{24} \mathrm{~A}$ beam of cold anions is mass-selected using a time-of-flight mass spectrometer, and the mass-selected anions are photodetached with a pulsed laser. A small fraction $\left(10^{-4}\right)$ of the ejected photoelectrons is energy-analyzed by time-of-flight. The electron energy resolution is 8 $\mathrm{meV}\left(64 \mathrm{~cm}^{-1}\right)$ for electrons with $0.65 \mathrm{eV}$ of kinetic energy and degrades as $(\mathrm{KE})^{3 / 2}$. The spectra below were obtained with third and fourth harmonics of a Nd:YAG laser $(20 \mathrm{~Hz}$ repetition rate) at $355 \mathrm{~nm}(3.49 \mathrm{eV})$ and $266 \mathrm{~nm}(4.66 \mathrm{eV})$, respectively, and each was signal-averaged for 250,000 laser shots. The laser polarization was set at the 'magic angle' $\left(\theta=54.7^{\circ}\right)$ with respect to the direction of electron detection. This eliminates any intensity effects due to anisotropy in the electron angular distribution.

The silicon cluster anions are generated with a laser-vaporization/ pulsed molecular beam source. $^{25}$ In this source, the output of a $\mathrm{XeCl}$ excimer laser (308 nm, 5-15 $\mathrm{mJ} / \mathrm{pulse}$ ) is focused onto the surface of a rotating and translating silicon rod. The 
resulting plasma is entrained in a gas pulse of He from a pulsed beam valve and passes through a $0.25 \mathrm{~cm}$ diameter, $1.25 \mathrm{~cm}$ long channel before expansion into the vacuum chamber. The He backing pressure behind the pulsed valve $(0.05 \mathrm{~cm}$ diameter orifice) is typically 100 psig. This source configuration produces $\mathrm{Si}_{\mathrm{n}}$ - clusters with $\mathrm{n} \leq 15$.

\section{Results and Discussion}

The photoelectron spectra of $\mathrm{Si}_{3}$ - obtained at $355 \mathrm{~nm}$ and $266 \mathrm{~nm}$ are shown in Figures 1a and $1 \mathrm{~b}$. The $355 \mathrm{~nm}$ spectrum shows two distinct bands, labelled $\mathrm{X}$ and $\mathrm{B}$. The $\mathrm{X}$ band shows a resolved vibrational progression of nine peaks with an average spacing of $360 \pm 40 \mathrm{~cm}^{-1}$, while the B band consists of a single sharp (20 meV FWHM) peak. In the $266 \mathrm{~nm}$ spectrum, a band between the $\mathrm{X}$ and $\mathrm{B}$ bands becomes apparent. This band, labelled A, consists of five peaks with an average spacing of $480 \pm 40 \mathrm{~cm}^{-1}$. In addition, two more bands labelled $C$ and $D$ appear at the higher photon energy. The C band shows extended, irregular vibrational structure, while the $\mathrm{D}$ band consists of two peaks separated by $480 \mathrm{~cm}^{-1}$. We expect the cluster anions to be sufficiently cold that the peak spacings in each band correspond to vibrational frequencies in the neutral cluster, rather than to anion frequencies due to 'hot band' transitions.

Assuming these transitions originate from the ground electronic state of $\mathrm{Si}_{3}{ }^{-}$, each band corresponds to a different electronic state of $\mathrm{Si}_{3}$, with the $\mathrm{X}$ band corresponding to the ground state. The estimated origin of each band is indicated by an arrow. From the $\mathrm{X}$ band origin in Figure 1a, we obtain $2.33 \mathrm{eV}$ for the electron affinity of $\mathrm{Si}_{3}$. This assumes that the weak signal at electron energies $>1.16 \mathrm{eV}$ is due to transitions from vibrationally excited $\mathrm{Si}_{3}-$. The energies of the excited $\mathrm{Si}_{3}$ electronic states relative to the 
ground state are then $\mathrm{A}(0.45 \mathrm{eV}), \mathrm{B}(0.89 \mathrm{eV}), \mathrm{C}(1.10 \mathrm{eV})$, and $\mathrm{D}(1.67 \mathrm{eV})$.

The photoelectron spectra of $\mathrm{Si}_{4}-$ obtained at $355 \mathrm{~nm}$ and $266 \mathrm{~nm}$ are shown in Figures $2 \mathrm{a}$ and $2 \mathrm{~b}$. We observe four bands corresponding to transitions to various electronic states of the neutral tetramer. The electron affinity of the $\mathrm{Si}_{4}$ ground state, labelled $\mathrm{X}$, is $2.15 \mathrm{eV}$, and the three excited states $\mathrm{A}, \mathrm{B}$, and $\mathrm{C}$ lie $0.81 \mathrm{eV}, 1.45 \mathrm{eV}$, and $2.01 \mathrm{eV}$, respectively, above the ground state. In the $355 \mathrm{~nm}$ spectrum, the $\mathrm{X}$ band is considerably narrower than the $\mathrm{A}$ band. The $\mathrm{X}$ band exhibits a partially resolved vibrational progression of three peaks with frequency $360 \pm 25 \mathrm{~cm}^{-1}$, and the $\mathrm{A}$ band shows a well-resolved progression of 10 peaks with frequency $300 \pm 25 \mathrm{~cm}^{-1}$. In the 266 $\mathrm{nm}$ spectrum, the $\mathrm{B}$ band shows irregular vibrational structure, while a four peak progression with frequency $450 \pm 65 \mathrm{~cm}^{-1}$ is observed in the $C$ band.

It is clear that assignment of the nature of the transition observed in our spectra from electronic sate energies and vibrational frequencies is impossible. However if we compare this limited spectroscopic information to previous ab initio calculations of the electronic energies and vibrational frequencies in neutral $\mathrm{Si}_{3}$ and $\mathrm{Si}_{4},{ }^{15,16,18,19}$ as well as the more recent calculated ground state geometries for $\mathrm{Si}_{3}{ }^{-}$and $\mathrm{Si}_{4}{ }^{-22}$ This comparison $^{26}$ resulted only in qualitative agreement between the theory and the experiment, so the definite assignment of the nature of the low-lying electronic states in the two clusters was far from definite. Recently, prompted by our results, Rohlfing and Raghavachari ${ }^{27}$ have recalculated the electronic state energies and geometries for $\mathrm{Si}_{3}$ and $\mathrm{Si}_{4}$, which agree not only qualitatively but also quantitatively as well. Both our experimental and these recent $a b$ initio are summarized in tables 1 and 2 . In the 
discussion that follows we will interpret our spectra using the results of this recent calculation. Although we cannot be certain which vibrational modes are responsible for the observed progressions, photoelectron spectra are usually dominated by progressions in totally symmetric modes, with the length of the progressions deternined by the geometry change between the anion and neutral.

We first consider the silicon tetramer. A planar rhombus geometry with $D_{2 h}$ symmetry is predicted for both the anion ${ }^{2} B_{2 g}$ and ncutral ${ }^{1} A_{g}$ ground states. The bond lengths differ by only $0.02 \AA$. The relatively short progression we observe in the $\mathrm{X}$ band is consistent with this small geometry change. In addition, the experimental frequency $\left(360 \mathrm{~cm}^{-1}\right)$ is close to the calculated frequency ${ }^{16}$ of $357 \mathrm{~cm}^{-1}$ for one of the totally symmetric modes of the neutral ${ }^{1} \mathrm{~A}_{\mathrm{g}}$ ground state. Thus, our spectra are consistent with the calculated anion and neutral ground states.

Rohlfing ${ }^{27}$ calculates the first excited state of $\mathrm{Si}_{4}$ to be a planar rhombic $\mathrm{D}_{2 \mathrm{~h}}$ species $\left({ }^{3} \mathrm{~B}_{3 \mathrm{u}}\right.$ term symbol) $0.99 \mathrm{eV}$ above the ground state which lies very close to the observed A-X spacing of 0.81 . The calculation also predicts that the diagonal bond length for this state will be $0.20 \AA$ longer than in the ground anion state. Hence, photodetachment to this state from the $\mathrm{X}^{2} \mathrm{~B}_{2 g}$ anion state should yield extended vibrational progressions, and band $\mathrm{A}$ is considerably more extended than the ground state progression. Thus, the A state appears to correspond nicely to the predicted ${ }^{3} A_{2}$ state. Further support comes from comparison of the predicted and observed vibrational frequencies. The calculated frequency for the totally symmetric mode of the ${ }^{3} \mathrm{~B}_{3 u}$ state $306 \mathrm{~cm}^{-1}$, and agrees remarkably well with the experimental A state frequency of $300 \mathrm{~cm}^{-1}$. 
The next excited state of $\mathrm{Si}_{4}$ predicted by Rohlfing and Raghavachari has a planar rectangular geometry $\mathrm{C}_{2 \mathrm{~h}}\left({ }^{3} \mathrm{~B}_{\mathrm{g}}\right.$ term symbol), and lies $1.65 \mathrm{eV}$ above the ground state and the vibrational frequencies for the three totally symmetric modes of this state are $167 \mathrm{~cm}$ ' ', $363 \mathrm{~cm}^{-1}$ and $567 \mathrm{~cm}^{-1}$. Once again this energy agrees with the B-X observed splitting, and the extended vibrational progression observed for this band is expected for a transition between a $D_{2 h}$ anion state and a $C_{2 h}$ neutral state. However the vibrational progression observed for this band is very irregular and not well resolved so the assignment of this band to an ${ }^{3} \mathrm{~B}_{\mathrm{g}}\left(\mathrm{C}_{2 h}\right)$ state is not definite.

We believe that there are two reasons for the congested appearance of band $\mathbf{B}$. It is possible that we are exciting several of the totally symmetric modes of this state, and since the predicted vibrational frequencies of these modes differ by about $150-200 \mathrm{~cm}^{-1}$, the resolution of our spectrometer will not allow us to resolve the closely separated transitions. Alternatively, since the calculation does predict the existence of a singlet excited electronic state at $1.70 \mathrm{eV}$, band $\mathrm{B}$ might could correspond to a transition to two distinct, close-lying electronic states of the neutral. Using similar argument to those presented above, we assign band $C$ to a transition to a ${ }^{3} B_{1 u}\left(D_{2 n}\right)$ neutral excited state.

The interpretation of the $\mathrm{Si}_{3}{ }^{-}$spectrum is more complicated. $\mathrm{Si}_{3}$ - is predicted ${ }^{22}$ to have a ${ }^{2} \mathrm{~A}_{1}$ ground state with $\mathrm{C}_{2 \mathrm{v}}$ symmetry and a bond angle of $66^{\circ}$. The calculations on $\mathrm{Si}_{3}$ predict a ${ }^{1} \mathrm{~A}_{1} \mathrm{C}_{2 v}$ ground state with a bond angle near $78^{\circ}$ and a low-lying ${ }^{3} \mathrm{~A}_{2}{ }^{\prime} \mathrm{D}_{3 \mathrm{~b}}$ excited state which lies between $0.03 \mathrm{eV}^{16}$ and $0.16 \mathrm{eV}^{19}$ above the ground state. This suggests that the $\mathrm{X}$ band consists of two overlapping electronic states, a definite possibility considering the irregular appearance of the band. However, the $\mathrm{X}$ band 
vibrational frequency of $360 \mathrm{~cm}^{-1}$ disagrees with the calculated frequencies, $148 \mathrm{~cm}^{-1}$ and $551 \mathrm{~cm}^{-1}{ }^{27}$ for the totally symmetric modes of the ${ }^{2} A_{1}$ ground state, and with the calculated value ${ }^{15}$ of $528 \mathrm{~cm}^{-1}$ for the totally symmetric mode in the ${ }^{3} \mathrm{~A}_{2}$ ' state. Similarly the calculation believes that the a state is a ${ }^{1} B_{2}$, however as for the ground state the agreement with the experiment is not impressive.

For band $B$ the agreement considerably better although not enough. The calculation predicts this state to be a ${ }^{3} A_{1}\left(C_{2 v}\right)$, with negligible bondlength and angle change with respect to the ground state of the anion. This explains the sharply peaked appearance of the band, however since no vibrational excitation of the state was observed, the assignment once again remains speculative.

\section{Conclusion}

The $\mathrm{Si}_{4}-$ photoelectron spectrum is qualitatively consistent with the predicted $\mathrm{D}_{2 \mathrm{~h}}$ ground state geometries for $\mathrm{Si}_{4}{ }^{-}$and $\mathrm{Si}_{4}$. The correspondence between experiment and theory is not as clear for the trimer or the excited states of the tetramer. Our results stimulate higher level $a b$ initio studies of these species, however the definite assignment of some the low-lying electronic states is not complete. On the experimental side, we plan to investigate these clusters at higher resolution $\left(3-5 \mathrm{~cm}^{-1}\right)$ using our negative ion threshold photodetachment spectrometer ${ }^{28}$ This should provide considerably more information on the vibrational frequencies and low-lying electronic states of small silicon clusters. 


\section{Figure Captions}

1. Photoelectron spectra of $\mathrm{Si}_{3}{ }^{2}$ at $355 \mathrm{~nm}(3.49 \mathrm{eV})$. Estimated band origins are indicated with arrows. The inset in shows the $\mathrm{X}$ and $\mathrm{A}$ bands magnified in intensity by a factor of 10. The A band origin is obtained from the $255 \mathrm{~nm}$ spectrum.

2. Photoelectron spectra of $\mathrm{Si}_{3}{ }^{3}$ at $266 \mathrm{~nm}(4.66 \mathrm{eV})$. Estimated band origins are indicated with arrows.

3. Photoelectron spectra of $\mathrm{Si}_{4}{ }_{4}$ at $355 \mathrm{~nm}(3.49 \mathrm{eV})$. Estimated band origins are indicated with arrows.

2. Photoelectron spectra of $\mathrm{Si}_{4}$ at $266 \mathrm{~nm}(4.66 \mathrm{eV})$. Estimated band origins are indicated with arrows.

Table Captions

1. Experimental and $a b$ initio spectroscopic constansts for $\mathrm{Si}_{3}{ }^{-}$and $\mathrm{Si}_{3}$.

2. Experimental and ab initio spectroscopic constants for $\mathrm{Si}_{4}{ }^{-}$and $\mathrm{Si}_{4}$. 
Table 1:

\begin{tabular}{|c|c|c|c|c|c|c|}
\hline & Peak & State & $\begin{array}{c}\mathrm{T}_{\mathrm{e}}(\mathrm{eV}) \\
\text { (ab initio) }\end{array}$ & $v\left(\mathrm{~cm}^{-1}\right)$ & $\begin{array}{c}\mathrm{T}_{\mathrm{e}}(\mathrm{eV}) \\
\text { Expt. }\end{array}$ & $V\left(\mathrm{~cm}^{-1}\right)$ \\
\hline \multirow{5}{*}{$\mathrm{Si}_{3}$} & $X$ & ${ }^{1} A_{1}\left(C_{2 v}\right)$ & 0.0 & 148,551 & 0.0 & 360 \\
\hline & A & - & - & - & 0.45 & 480 \\
\hline & B & ${ }^{3} A_{1}\left(C_{2 v}\right)$ & 0.87 & 325,523 & 0.89 & - \\
\hline & $\mathrm{C}$ & ${ }^{3} B_{1}\left(C_{2 v}\right)$ & 1.22 & 247,481 & 1.10 & - \\
\hline & $D$ & - & - & - & 1.67 & 480 \\
\hline
\end{tabular}

Table 2:

\begin{tabular}{|c|c|c|c|c|c|c||}
\hline & Peak & State & $\begin{array}{c}\mathrm{T}_{\mathrm{e}}(\mathrm{eV}) \\
(a b \text { initio })\end{array}$ & $v\left(\mathrm{~cm}^{-1}\right)$ & $\mathrm{T}_{\mathrm{e}}(\mathrm{eV})$ & $v\left(\mathrm{~cm}^{-1}\right)$ \\
\hline \multirow{6}{*}{$\mathrm{Si}_{4}$} & $\mathrm{X}$ & ${ }^{1} \mathrm{~A}_{8}\left(\mathrm{D}_{2 \mathrm{~h}}\right)$ & 0.0 & 357,489 & 0.0 & 360 \\
\cline { 2 - 7 } & $\mathrm{A}$ & ${ }^{3} \mathrm{~B}_{34}\left(\mathrm{D}_{2 \mathrm{~h}}\right)$ & 0.99 & 306 & 0.81 & 300 \\
\cline { 2 - 7 } & $\mathrm{B}$ & ${ }^{3} \mathrm{~B}_{\mathrm{g}}\left(\mathrm{C}_{2 \mathrm{~h}}\right)$ & 1.65 & $167,394,561$ & 0.89 & - \\
\hline
\end{tabular}


Intensity

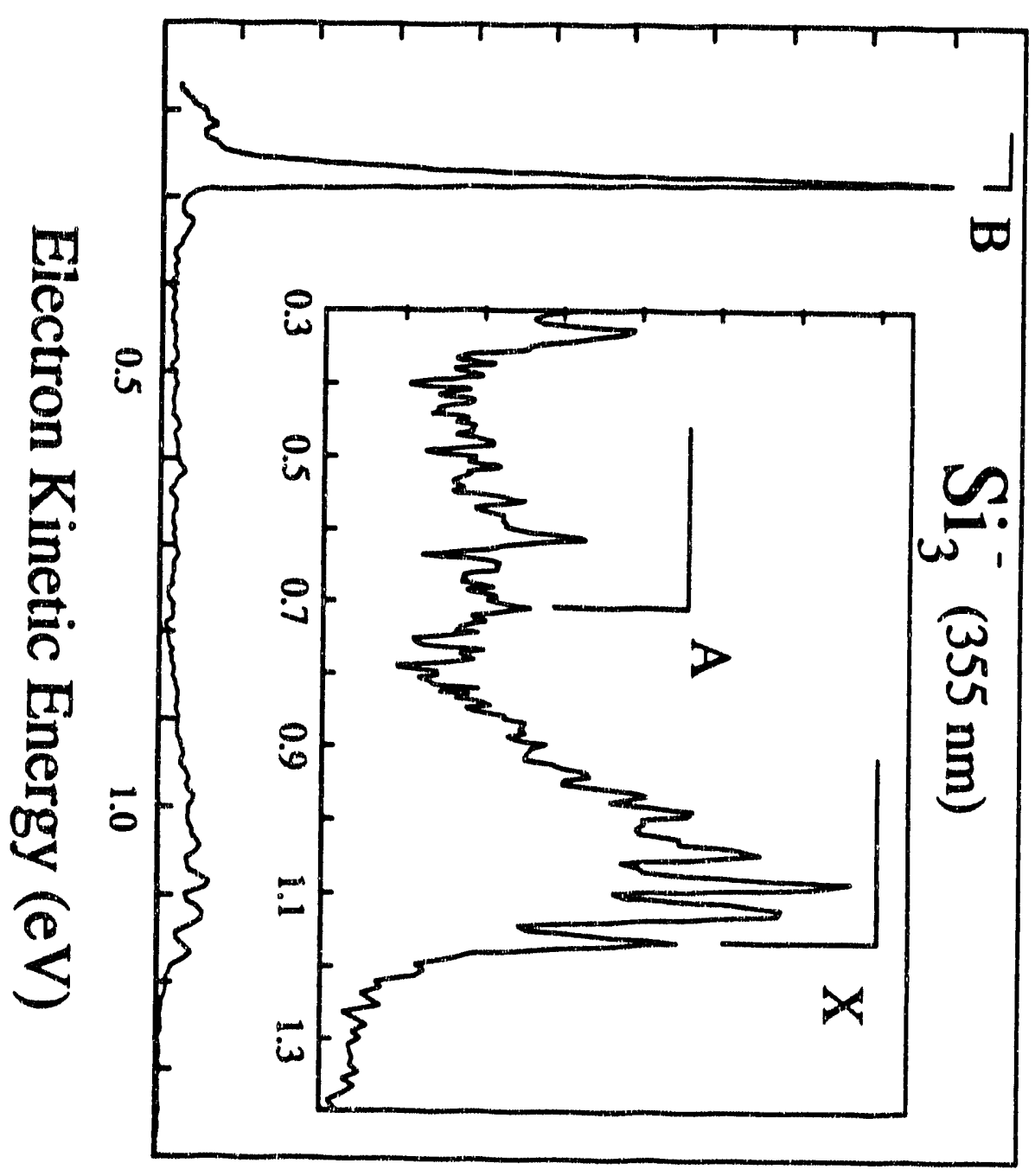

Figure 1 


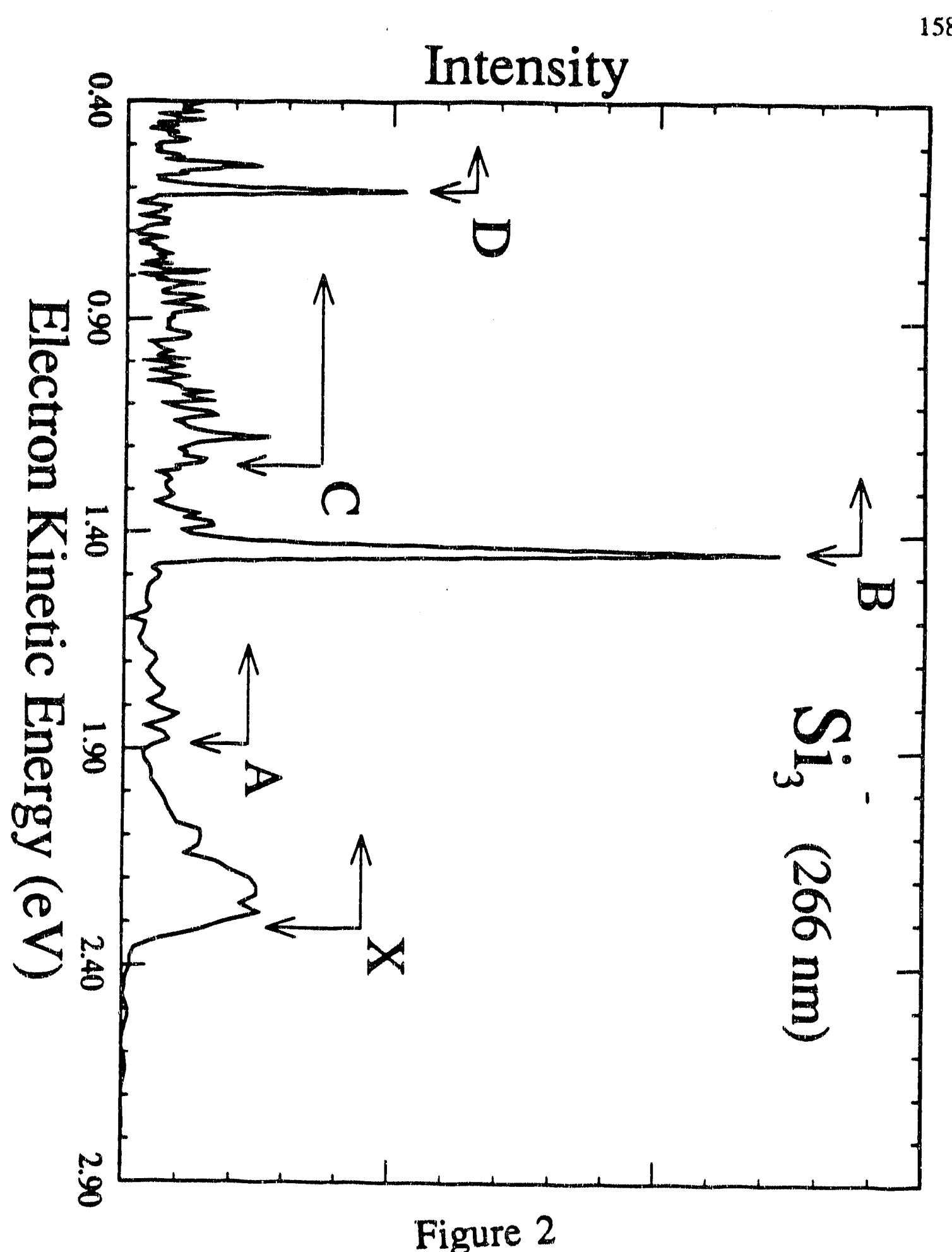




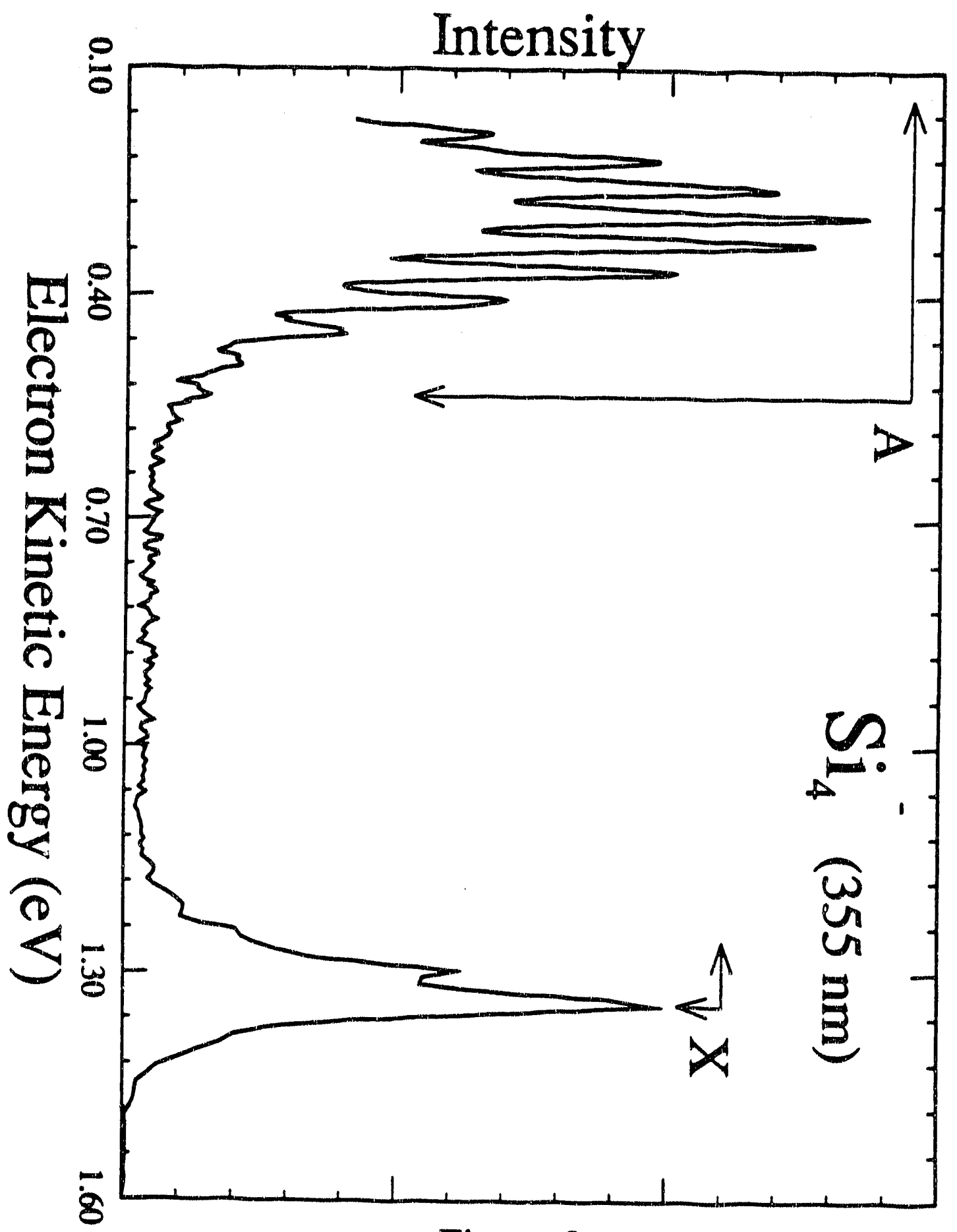

Figure 3 


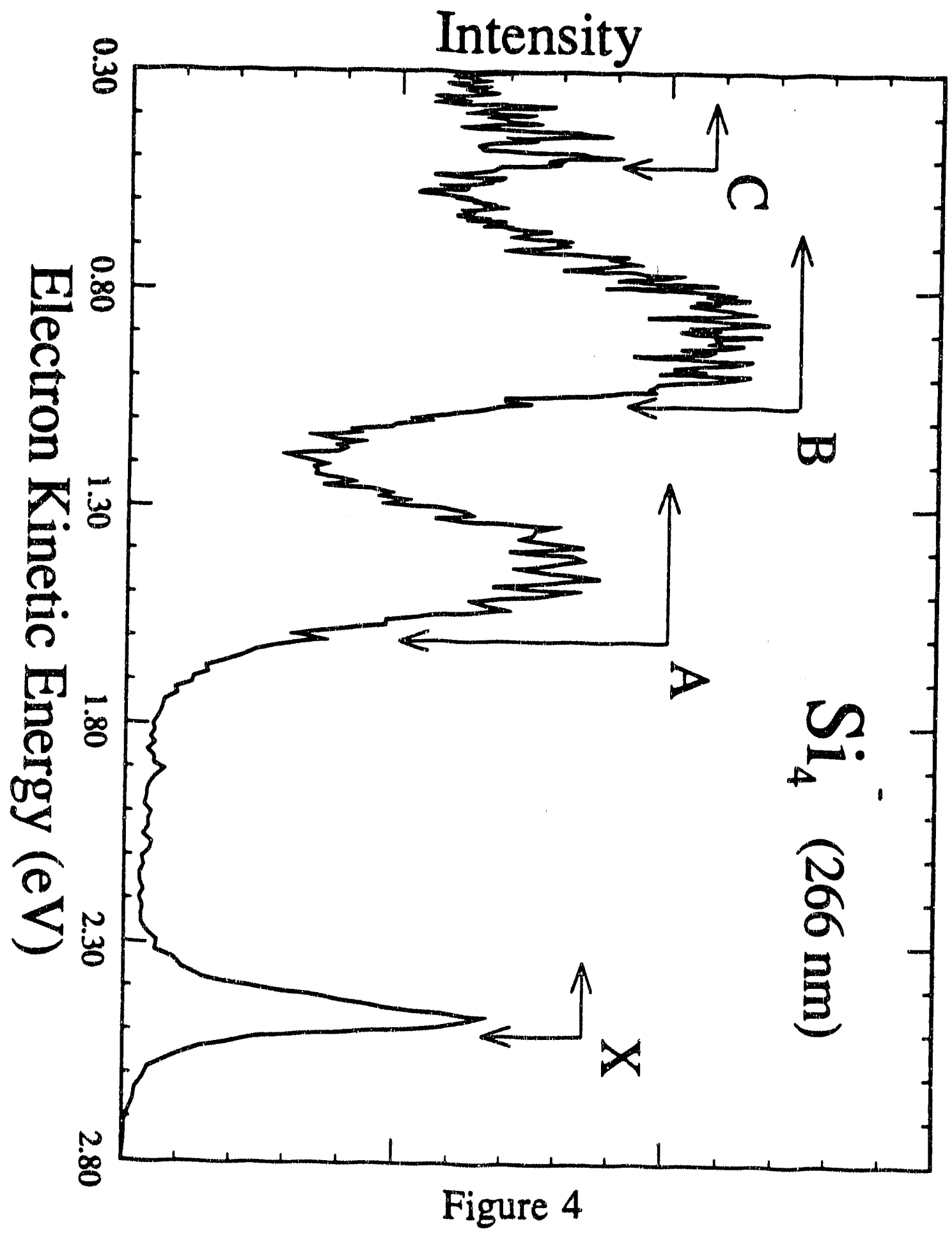




\section{References}

1. A. Herrmann, M. Hofmann, S. Leutwyler, E. Schumacher, and L. Wöste, Chem. Phys. Lett. 62, 216 (1979); G. Delacrétaz, E. R. Grant, R. L. Whetten, L. Wöste, and J. W. Zwanziger, Phys. Rev. Lett. 62, 2598 (1986).

2. M. D. Morse, J. B. Hopkins, P. R. R. Langridge-Smith, and R. E. Smalley, J. Chem. Phys. 79, 5316 (1983); W. H. Crumley, J. S. Hayden, and J. L. Gole, J. Chem. Phys. 84, 5250 (1986); E. A. Rohlfing and J. J. Valentini, Chem. Phys. Lett. 126, 113 (1986).

3. P. Y. Cheng and M. A. Duncan, Chem. Phys. Lett. 152, 341 (1988).

4. Z. Fu, G. W. Lemire, Y. M. Hamrick, S. Taylor, J.-C. Shui, and M. D. Morse, J. Chem. Phys. 88, 3524 (1988).

5. K. M. Ervin, J. Ho, and W. C. Lineberger, J. Chem. Phys. 89, 4514 (1988).

6. M. F. Jarrold and K. M. Creegan, Chem. Phys. Lett. 166, 116 (1990).

7. P. F. Bernath, K. H. Kinkle, and J. J. Keady, Science 244, 562 (1989); J. R. Heath, A. L. Cooksy, M. H. W. Gruebele, C. A. Schmuttenmaer, and R. J. Saykally, Science 244, 564 (1989); N. Moazzen-Ahmadi, A. R. W. McKellar, and T. Amano, J. Chem. Phys. 91, 2140 (1989).

8. D. G. Leopold, J. Ho, and W. C. Lineberger, J. Chem. Phys. 86, 1715 (1987).

9. O. Cheshnovsky, S. H. Yang, C. L. Pettiette, M. J. Craycraft, Y. Liu, and R. E. Smalley, Chem. Phys. Lett. 138, 119 (1987).

10. S. H. Yang, C. L. Pettiette, J. Conceicao, O. Cheshnovsky, and R. E. Smalley, Chem. Phys. Lett. 139, 233 (1987); S. Yang, K. J. Taylor, M. J. Craycraft, J. Conceicao, 
C. L. Pettiette, O. Cheshnovsky, and R. E. Smalley, Chem. Phys. Lett. 144, 431 (1988).

11. K. J. Taylor, C. L. Pettiette, M. J. Craycraft, O. Cheshnovsky, and R. E. Smalley, Chem. Phys. Lett. 152, 347 (1988).

12. K. M. McHugh, J. G. Eaton, G. H. Lee, H. W. Sarkas, L. H. Kidder, J. T. Snodgrass, M. R. Manaa, and K. H. Bowen, J. Chem. Phys. 91, 3702 (1989).

13. G. Gantefor, M. Gausa, K. H. Meiwes-Bröer, and H. O. Lutz, Z. Phys. D 9, 253 (1988); Faraday Discuss. Chem. Soc. 86, 197 (1988).

14. G. H. F. Diercksen, N. E. Gruner, J. Oddershede, and J. R. Sabin, Chem. Phys. Lett. 117, 29 (1985).

15. R. S. Grev and H.F. Schaefer, Chem. Phys. Lett. 119, 111 (1985).

16. K. Raghavachari, J. Chem. Phys. 83, 3520 (1985); J. Chem. Phys. 84, 5672 (1986).

17. D. Tomanek and M. A. Schluter, Phys. Rev. Lett. 56, 1055 (1986).

18. G. Pacchioni and J. Koutecky, J. Chem. Phys. 84, 3301 (1986).

19. K. Balasubramanian, Chem. Phys. Lett. 125, 400 (1986); 135, 283 (1987).

20. P. Ballone, W. Andreoni, R. Car, and M. Parrinello, Phys. Rev. Lett. 60, 271 (1988).

21. K. Raghavachari and C. M. Rohlfing, J. Chem. Phys. 89, 2219 (1988).

22. C. M. Rohlfing and K. Raghavachari,J. Chem. Phys. 94, 3670 (1991).

23. R. B. Metz, A. Weaver, S. E. Bradforth, T. N. Kitsopoulos, and D. M. Neumark, J. Phys. Chem. 94, 1377 (1990).

24. L. A. Posey, M. J. Deluca, and M. A. Johnson, Chem. Phys. Lett. 131, 170 (1986). 
25. T. G. Dietz, M. A. Duncan, D. E. Powers, and R. E. Smalley, J. Chem. Phys. 74, 6511 (1981); V. E. Bondebey and J. H. English, J. Chem. Phys. 74, 6978 (1981).

26. T.N. Kitsopoulos, C.J. Chick, A. Weaver, and D.M. Neumark, J. Chem. Phys. 93, 6108 (1990).

27. C.M. Rohlfing and K. Raghavachari, J. Chem. Phys. in press (1991).

28. T. N. Kitsopoulos, I. M. Waller, J. G. Loeser, and D. M. Neumark, Chem. Phys. Lett. 159, 300 (1989). 


\section{Chapter VI}

\section{Threshold photodetachment spectroscopy of $\mathrm{C}_{5}-$}

\section{Introduction}

The importance of carbon clusters in astrophysics and combustion has made them the focal point of intense experimental effort. Carbon clusters come in all shapes and sizes, from linear chains and closed ring structures, to spheroidal fullerenes like $C_{60}$. The spectroscopy of these intriguing species has been reviewed by Weltner and Van Zee. ${ }^{1}$ Although a great deal of progress has been made in understanding the physical and chemical properties of these clusters there remain many unanswered questions concerning the nature of the electronic, vibrational and rotational structure of these species.

Over the years negative ion photoelectron spectroscopy (PES) has offered a very promising technique for studying clusters. ${ }^{2,3,4,5}$ The first ultraviolet photoelectron spectra for carbon clusters $\mathrm{C}_{\mathrm{n}=2 \cdot 29}{ }^{-}$, were obtained by Smalley and co-workers ${ }^{6}$ at a resolution of about $1000 \mathrm{~cm}^{-1}$, which allowed electronic bands to be mapped out but no vibrational progressions. More recently, Arnold et al. ${ }^{7}$ have obtained vibrationally resolved photoelectron spectra of $\mathrm{C}_{\mathrm{n}=2 \cdot 11}$ - at a resolution of about $100 \mathrm{~cm}^{-1}$. This resolution is comparable to the lowest vibrational frequencies for these clusters, and not all features in the spectra were cleanly resolved. In this Communication, we report a considerably higher resolution $\left(6-10 \mathrm{~cm}^{-1}\right)$ photodetachment study of $\mathrm{C}_{5}$ - using threshold photodetachment spectroscopy. ${ }^{8,9}$ We observe considerably more vibrational structure than in the $\mathrm{C}_{5}$ - photoelectron spectrum and obtain evidence for a low-lying excited electronic state of $\mathrm{C}_{5}$. 


\section{Experimental}

The threshold photodetachment spectrometer used in this study is described in detail elsewhere. ${ }^{8,10}$ A beam of cold anion carbon clusters is generated in a laser vaporization/pulsed molecular beam source and is mass-selected using time of flight. The cluster of interest is then photodetached using a excimer-pumped tunable dye laser operating at $20 \mathrm{~Hz}$, and electrons with near zero kinetic energy (ZEKE) are collected as a function of photon energy. The selective detection of the ZEKE electrons is done using the method developed by Muller-Dethlefs et al. ${ }^{11}$ In the spectra shown here, the electron signal was averaged for 1000 laser shots at each wavelength and normalized to both the laser power and the ion current. Four different laser dyes were utilized to obtain the $\mathrm{C}_{5}$ spectrum: Stilbene $3(418 \mathrm{~nm}-440 \mathrm{~nm})$, Exalite $416(408 \mathrm{~nm}-427 \mathrm{~nm})$, DPS (397 nm $414 \mathrm{~nm})$ and BiBuQ $(375 \mathrm{~nm}-400 \mathrm{~nm})$.

\section{Results and Discussion}

Figure 1(a) shows the $\mathrm{C}_{5}{ }^{-}$threshold photodetachment spectrum in which the cluster beam was seeded in UHP helium; Figure 1(b) shows a smaller region of this spectrum taken with neon as the seed gas. Two types of peaks appear in Figure 1(a): peaks a - F are all between 10 and $30 \mathrm{~cm}^{-1}$ wide, while a series of broader peaks (FWHM $\sim 100 \mathrm{~cm}^{-1}$ ) appears at slightly higher energy. The two sets of peaks are labelled bands $\mathbf{X}$ and $\mathbf{a}$, respectively. Peaks in each band represent transitions between vibrational levels of $\mathrm{C}_{5}{ }^{-}$and $\mathrm{C}_{5}$. The peaks in band $\mathrm{X}$ can be further grouped together according to their shape: peaks a, A, D, and $\mathrm{E}$ which are doublets split by $22 \mathrm{~cm}^{-1}$, and peaks b, B, c, C and $F$ which are single peaks about $30 \mathrm{~cm}^{-1}$ wide. 
Band $\mathbf{X}$ is the lowest energy band observed and presumably results from transitions betweem the ground electronic states of the anion and neutral cluster. It is dominated by peak $A$, which is assigned to the $C_{5}\left(v^{\prime}=0\right) \leftarrow C_{5}{ }^{-}\left(v^{\prime \prime}=0\right)$ vibrational transition. The dominance of a single peak implies a small geometry change between the anion and the neutral cluster. Several high resolution infrared experiments ${ }^{12,13,24}$ have determined the structure of $C_{5}$ to be linear, in agreement with the prediction of Pitzer and Clementi, ${ }^{15}$ and our spectrum indicates $\mathrm{C}_{5}{ }^{-}$is linear as well. As mentioned above, peak A is actually two peaks separated by $22 \mathrm{~cm}^{-1}$. Molecular orbital considerations show that $\mathrm{C}_{5}$ has a ${ }^{1} \Sigma_{\mathrm{B}}{ }^{+}$ground state and that the LUMO in $C_{5}$ is a $\pi_{\mathrm{u}}$ orbital, so $\mathrm{C}_{5}{ }^{-}$is expected to have a ${ }^{2} \Pi_{u}$ ground state. We assign the $22 \mathrm{~cm}^{-1}$ splitting to the spltting between the ${ }^{2} \Pi_{1 / 2}$ and ${ }^{2} \Pi_{32}$ spin-orbit levels of the $C_{5} \cdot{ }^{2} \Pi_{4}$ state. The smaller $A_{2}$ peak observed at higher wavelength originates from the excited ${ }^{2} \Pi_{3 / 2}$ level and is less intense because the population of this level is lower in our cold ion beam. Based on the intensity ratio $A_{2} / A_{1}$, we obtain a 'spin-orbit temperature' of $60 \mathrm{~K}$ for the He seeded beam and $30 \mathrm{~K}$ for the Ne seeded beam. The transition energy of the larger $A_{1}$ peak, $2.854 \pm 0.001 \mathrm{eV}$, is the electron affinity of $\mathrm{C}_{5}$.

Considering the other peaks in band $\mathbf{X}$, we find that intensities for peaks $\mathrm{a}, \mathrm{b}$, and c vary considerably with the ion source conditions and we assign them to 'hot bands' originating from vibrationally excited $\mathrm{C}_{5}{ }^{-}$. These hot band transitions are absent from the $1 \mathrm{~b}$ spectrum suggesting, in accordance with the spin-orbit temperatures, that the clusters seeded in $\mathrm{Ne}$ expansions are colder than those seeded in He. The remaining peaks are transitions from $\mathrm{C}_{5}-\left(v^{\prime \prime}=0\right)$ to vibrationally excited $\mathrm{C}_{5}$. Their assignments, listed 
in Table I, are aided by $a b$ initio calculated vibrational frequencies ${ }^{16}$ and the infrared absorption work on $\mathrm{C}_{5}$ by Amano and co-workers. ${ }^{14}$ We observe transitions to the $\mathbf{v}^{\prime}=$ 1 level of the $v_{2}$ symmetric stretch and to the $v^{\prime}=2$ levels of the $v_{5}, v_{6}$, and $v_{7}$ bending modes (since the bending modes are not totally symmetric, only transitions to even levels from the $v^{\prime \prime}=0$ anion level can occur $\left.{ }^{17}\right)$. We obtain the following $C_{5}$ vibrational frequencies: $v_{2}=779 \mathrm{~cm}^{-1}, v_{5}=216 \mathrm{~cm}^{-1}, v_{6}=535 \mathrm{~cm}^{-1}, v_{7}=106 \mathrm{~cm}^{-1}$. These agree reasonably well with the $a b$ initio frequencies of Raghavachari and Binkley ${ }^{16}$ scaled by a factor of 0.89 , the suggested correction factor for Hartree-Fock calculations. ${ }^{18}$

From the hot band positions in our spectrum, we can attempt to learn about the vibrational frequencies of $\mathrm{C}_{5}-$. Assuming the spin orbit temperature of $60 \mathrm{~K}$ in Figure $1 \mathrm{a}$ is representative of the anion vibrational temperature, then, based on Raghavachari's recent calculation of the $\mathrm{C}_{5}{ }^{-}$vibrational frequencies, ${ }^{19}$ only the lowest frequency vibrational mode will have non-negligible excitation. This is the $v_{7} \pi_{u}$ bending mode, the frequency of which is calculated to be $162 \mathrm{~cm}^{-1} .{ }^{20}$ In our $C_{5}$ spectrum the $0 \leftarrow 0$ transition is by far the most intense, so we expect the largest hot band, peak a, to correspond to a $v_{7}{ }^{\prime}=1 \leftarrow v_{7}{ }^{\prime \prime}=1$ transition. Peaks a and $b$ are separated by twice the frequency of the $v_{7}$ mode in $C_{5}$. We therefore assign peak $b$ to the $v_{7}{ }^{\prime}=3 \leftarrow v_{7}{ }^{\prime \prime}=1$ transition. Peak a is located $94 \mathrm{~cm}^{-1}$ to the red of A from which we find $v_{7}=200 \mathrm{~cm}^{-1}$ for $\mathrm{C}_{5}{ }^{-}$. This value is close to but somewhat higher than the $a b$ initio value.

An interesting feature of band $\mathbf{X}$ is that the peak shapes vary considerably. Peak E, which corresponds to a transition involving the totally symmetric $v_{2}$ mode, is a doublet, split by the energy difference between the ${ }^{2} \Pi_{1,2}$ and ${ }^{2} \Pi_{3 / 2}$ anion spin orbit states. In 
contrast, peaks B, C, and F appear as singlets; each of these peaks corresponds to a transitions to the $v^{s}=2$ level of a degenerate bending mode of $C_{5}$. The width of these peaks is about $30 \pm 10 \mathrm{~cm}^{-1}$ and is considerably larger than the instrumental resolution under which the spectrum was obtained. We believe these peaks consist of closely spaced overlapping transitions and offer the following explanation.

For a linear molecule such as $C_{5}$ in a ${ }^{1} \Sigma_{8}^{+}$electronic state, the excitation of 2 quanta in a degenerate vibrational mode results in two states of symmetry $\Sigma$ and $\Delta$ with vibrational angular momentum $l=0$ and 2 , respectively. These are degenerate for a two dimensional harmonic oscillator, but anharmonicity can remove this degeneracy. If the splitting of these vibrational levels in $C_{5}$ is comparable to the spin orbit splitting in $C_{5}-$, then the $v^{\prime}=2 \leftarrow v^{\prime \prime}=0$ photcletachment transition could be composed of two overlapping pairs of transitions, namely the $\Sigma \leftarrow{ }^{2} \Pi_{1 / 2,3 / 2}$ and $\Delta \leftarrow{ }^{2} \Pi_{1 / 2,3 / 2}$ transitions, yielding a single broad feature in the spectrum. The second pair of transitions (to the $C_{5} \Delta$ state) is allowed only if there is vibronic coupling in the anion. ${ }^{21}$ The extent of this coupling is unknown, but we note that similar (although not identical) effects have been seen in the in the ${ }^{2} \Sigma \leftarrow{ }^{2} \Pi$ electronic absorption spectra of $\mathrm{NCO},{ }^{22} \mathrm{BO}_{2},{ }^{23}$ and the ${ }^{1} \Sigma \leftarrow ' \Pi$ absorption spectrum of $\mathrm{C}_{3}{ }^{24}$

Further insight into the nature of the vibrational levels of $C_{s}$ is provided by the intensity and shape of peak D. This peak is assigned to the transition to the $2 v_{7}+2 v_{5}$ level of $C_{s}$ on the basis of its position. However, this peak is nearly as intense as peaks $B$ and $C$, which are transitions to the $2 v_{7}$ and $2 v_{5}$ vibrational levels, respectively, whereas a simple normal mode picture would lead one so expect a combination band such as peak 
$D$ to be considerably less intense. One possible explanation of the anomalous intensity is the assignment of this peak to a Fermi type resonance; ${ }^{25}$ the component of the $2 v_{7}$ $+2 v_{5}$ level with $\Sigma$ symmetry and vibrational angular momentum $l=0$ can interact with the nearby $v_{2}=1$ symmetric stretch level, and peak $D$ therefore borrows intensity from peak $E$, the $v^{\prime}=1 \leftarrow v^{\prime \prime}=0$ transition. Peak $D$ is a doublet split by $22 \mathrm{~cm}^{-1}$, the anion spin-orbit splitting, indicating that only transitions to a single $C_{5}$ vibrational state are occurring. This is consistent with the idea of a Fermi resonance in which only transitions to the $l=0$ component of the $2 v_{7}+2 v_{5}$ level borrow intensity. Hence unlike peaks $B$, $C$, and F, peak D appears as a doublet, rather than an unresolved quartet.

In Figure 1a, we observe a second band labeled a at higher energy than band $\mathbf{X}$. The peaks are noticeably broader in band $a$, and the most prominent peaks $(G, H, I)$ do not appear to be transitions to vibrationally excited levels of the $C_{5}$ ground electronic state. Instead, band a appears to be a transition to an excited electronic state of $C_{5}$.

If this state is linear, one can speculate on its nature by considering the molecular orbitals of $C_{5}$ and $C_{5}-$. The valence molecular orbital configuration for $C_{5}$ is $\sigma_{8}{ }^{2} \sigma_{u}{ }^{2} \pi_{8}{ }^{4} \pi_{u}{ }^{1}$. Removal of an electron from the HOMO $\left(\pi_{u}\right)$ yields the $\mathrm{X}^{\prime} \Sigma_{g}^{+}$ground electronic state of C.s. Similarly, the removal of other electrons will result in the formation of excited neutral electronic states. As we have discussed elsewhere, ${ }^{8,10}$ in threshold photodetachment spectroscopy we can only observe transitions which involve s-wave photodetachment, where the orbital angular momentum of the ejected electron is zero. Reed et al. ${ }^{26}$ have shown that for $D_{- \text {r. }}$ symmetry, s-wave photodetachment occurs only when the photoelectron is detached from either a $\pi_{u}$ or $\sigma_{u}$ orbital. Hence, if band $a$ is a 
transition to a linear excited electronic state, it must involve the removal of a $\sigma_{u}$ electron. The lowest state from the resulting $\sigma_{g}{ }^{2} \sigma_{u}{ }^{1} \pi_{8}{ }^{4} \pi_{u}{ }^{1}$ configuration is the $a^{3} \Pi_{8}$ state.

This assignment offers a qualitative explanation of the broad peaks in band $\mathbf{a}$. Assuming Hund's case (a) coupling, the $a^{3} \Pi_{g}$ state will split into three spin orbit components; all 6 possible transitions from the ${ }^{2} \Pi_{u}$ anion state are allowed and should occur. If the magnitude of the spin orbit splitting in the $a^{3} \Pi_{g}$ state is such that adjacent $a^{3} \Pi_{0,1,2} \leftarrow{ }^{2} \Pi_{1 / 2,3 / 2}$ transitions are too close in energy to be resolved, the six transitions will appear as an unresolved single peak, in agreement with our spectrum.

On the other hand, the vibrational structure in band a, while not assigned, looks more complex than expected for a linear anion $\rightarrow$ linear neutral transition. In addition to the major peaks $\mathrm{G}, \mathrm{H}$, and $\mathrm{I}$, we cbserve an extended progression of smaller peaks with an average separation of $200 \pm 50 \mathrm{~cm}^{-1}$. Another point to consider is that an ab initio MRD CI calculations by Pacchioni and Kouteck $y^{27}$ on linear excited electronic states of $C_{s}$ found the ${ }^{3} \Pi_{\mathrm{g}}$ state to lie $2.56 \mathrm{eV}$ higher than the ${ }^{1} \Sigma_{\mathrm{g}}{ }^{+}$state, whereas band a begins about $0.26 \mathrm{eV}$ above band $\mathrm{X}$ in our spectrum. Whether this substantial discrepancy arises from inaccuracies in the calculation or from an incorrect interpretation of our spectrum remains to be seen.

Another explanation of the peaks in band $a$ is that they result from autodetachment from a metastable excited electronic state of $\mathrm{C}_{5}-$. Recently, Adamowicz ${ }^{28}$ has predicted the existence of such a state $3.08 \mathrm{eV}$ above the $\mathrm{C}_{5}{ }^{-}$ground state and is optically accessible $\left(a^{2} \Sigma_{g}\right.$ state with configuration $\left.\sigma_{B}{ }^{2} \sigma_{u}{ }^{1} \pi_{B}^{4} \pi_{u}{ }^{2}\right)$. The predicted energy is right in the middle of band a. However, there are two reasons we believe band a is not due to 
autodetachment. First of all, the major peaks in band a do appear in our lower resolution photoelectron spectrum, ${ }^{7}$ although the intensity of these peaks is higher in the thresh-id spectrum. Secondly, electrons generated by the decay of an autodetaching anion state are, in general, going to have more than $10 \mathrm{~cm}^{-1}$ of kinetic energy and are normally discriminated against by our detection scheme. To check for autodetachment we collected all the electrons generated by the photodetachment, but no structure was observed in the region of band a. We therefore believe band a is from direct detachment to an excited state of $\mathrm{C}_{5}$, but the nature of this state is open to question.

\section{Conclusion}

We have obtained the vibrationally resolved threshold photodetachment of $\mathrm{C}_{5}-$, and have presented a preliminary assignment which explains many of the features in our spectrum. Further analysis which involves modelling the potentials for both the $C_{5}$ and $\mathrm{C}_{5}$ - ground and excited electronic states is necessary. We hope that our results will prompt more detailed theoretical work which will help us with our analysis. This report demonstrates that threshold photodetachment spectroscopy is a powerful technique for studying polyatomic clusters. We have recently obtained the threshold photodetachment spectra for other carbon clusters and will report on this in the near future. 


\section{Figure Caption}

1. (Top) Threshold photodetachment spectrum of $\mathrm{C}_{5}{ }^{-}$obtained in a He expansion. (Bottom) A section of the threshold photodetachment spectrum of $\mathrm{C}_{5}$ - obtained in a $\mathrm{Ne}$ expansion.

\section{Table Caption}

1. Energies and transition assingments for the peaks appearing in band $X$ of the threshold photodetachment spectrum of $\mathrm{C}_{5}-$ 
Table I:

\begin{tabular}{|c|c|c|}
\hline Peak & Position $\left(\mathrm{cm}^{-1}\right)$ & $\mathrm{C}_{5} \leftarrow \mathrm{C}_{5}-$ Transition Assignment \\
\hline$a_{2}$ & 22,903 & ${ }^{1} \Sigma_{g}^{+}\left(v_{7}{ }^{\prime}=1\right) \leftarrow{ }^{2} \Pi_{3 / 2}\left(v_{\gamma}{ }^{\prime \prime}=1\right)$ \\
\hline$a_{1}$ & 22,925 & ${ }^{1} \Sigma_{\mathrm{B}}^{+}\left(v_{7}{ }^{\prime}=1\right) \leftarrow{ }^{2} \Pi_{1 / 2}\left(v_{7}{ }^{\prime \prime}=1\right)$ \\
\hline $\mathrm{A}_{2}$ & 22,997 & ${ }^{1} \Sigma_{B}{ }^{+}\left(v^{\prime}=0\right) \leftarrow{ }^{2} \Pi_{3 / 2}\left(v^{\prime \prime}=0\right)$ \\
\hline$A_{1}$ & 23,019 & ${ }^{1} \Sigma_{g}^{+}\left(v^{\prime}=0\right) \leftarrow{ }^{2} \Pi_{1 / 2}\left(v^{\prime \prime}=0\right)$ \\
\hline b & 23,139 & ${ }^{1} \Sigma_{8}^{+}\left(v_{7}^{\prime}=3\right) \leftarrow{ }^{2} \Pi_{u}\left(v_{7}{ }^{\prime \prime}=1\right)$ \\
\hline B & 23,231 & ${ }^{1} \Sigma_{g}{ }^{+}\left(v_{7}{ }^{\prime}=2\right) \leftarrow{ }^{2} \Pi_{u}\left(v_{y}{ }^{\prime \prime}=0\right)$ \\
\hline C & 23,451 & ${ }^{1} \Sigma_{g}^{+}\left(v_{s}^{\prime}=2\right) \leftarrow{ }^{2} \Pi_{u}\left(v_{s}{ }^{\prime \prime}=0\right)$ \\
\hline $\mathrm{D}_{2}$ & 23,641 & ${ }^{1} \Sigma_{8}^{+}\left(v_{7}^{\prime}=2, v_{5}^{\prime}=2\right) \leftarrow{ }^{2} \Pi_{3 / 2}\left(v_{7}^{\prime \prime}=0, v_{5}^{\prime}=0\right)$ \\
\hline$D_{1}$ & 23,663 & ${ }^{1} \Sigma_{g}^{+}\left(v_{7}{ }^{\prime}=2, v_{5}{ }^{\prime}=2\right) \leftarrow{ }^{2} \Pi_{1 / 2}\left(v_{7}{ }^{\prime \prime}=0, v_{5}{ }^{\prime}=0\right)$ \\
\hline$E_{2}$ & 23,777 & ${ }^{1} \Sigma_{B}^{+}\left(v_{2}^{\prime}=1\right) \leftarrow{ }^{2} \Pi_{3 / 2}\left(v_{2}{ }^{\prime}=0\right)$ \\
\hline$E_{1}$ & 23,798 & ${ }^{1} \Sigma_{8}{ }^{+}\left(v_{2}{ }^{\prime}=1\right) \leftarrow{ }^{2} \Pi_{1 / 2}\left(v_{2}{ }^{\prime \prime}=0\right)$ \\
\hline $\mathrm{F}$ & 24,089 & ${ }^{1} \Sigma_{g}^{+}\left(v_{6}^{\prime}=2\right) \leftarrow{ }^{2} \Pi_{u}\left(v_{6}{ }^{\prime \prime}=0\right)$ \\
\hline
\end{tabular}



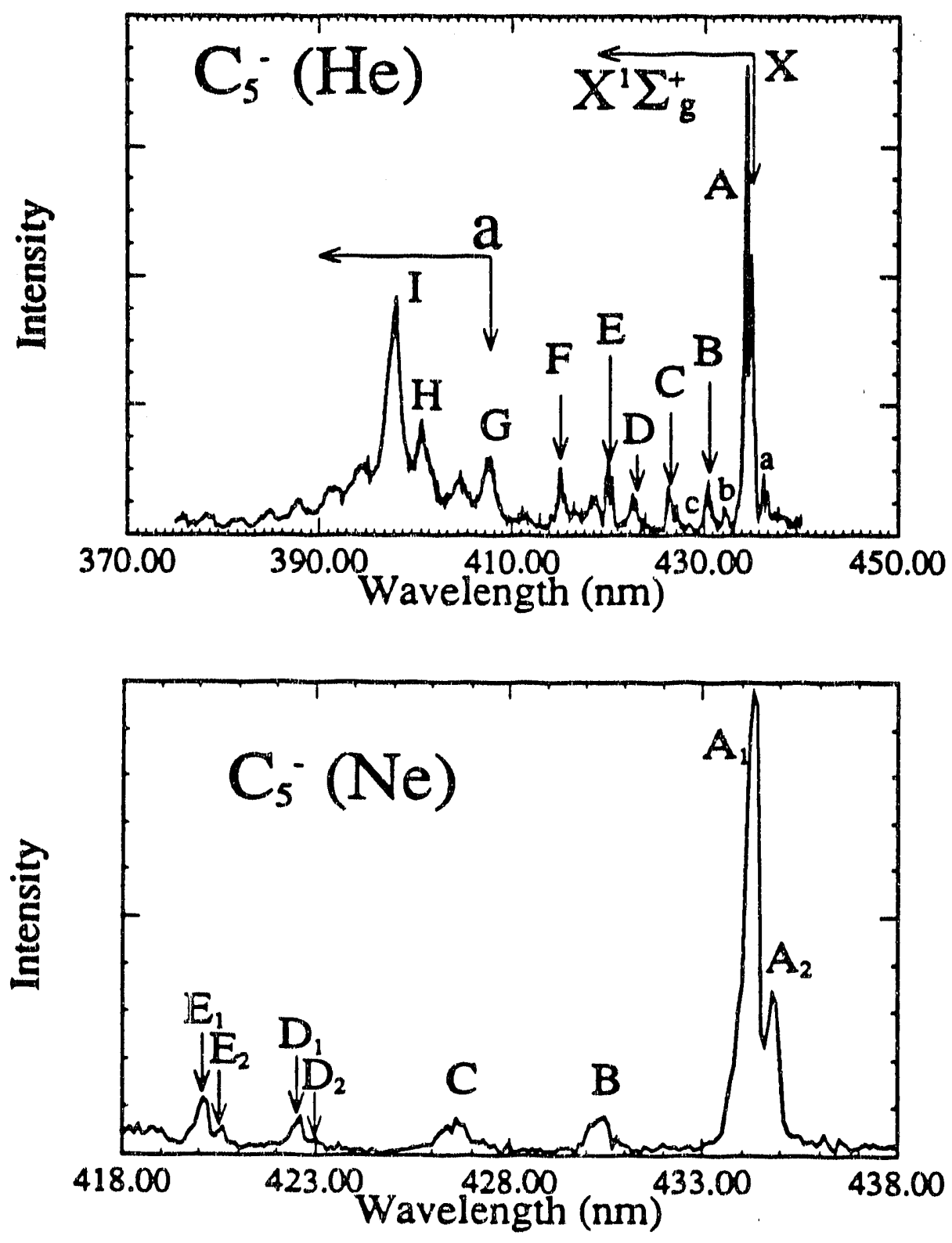

Figure 1 


\section{References}

1. W. Weltner,Jr. and R.J VanZee, Chem. Rev. 89, 1713 (1989).

2. J. Ho, K.M. Ervin, and W.C. Lineberger, J. Chem. Phys. 94, 3670 (1991).

3. L.A. Posey, M.J. Deluca, and M.A. Johnson, Chem. Phys. Lett. 131, 170 (1986); L.A. Posey and M.A. Johnson, J. Chem. Phys. 88, 5383 (1988).

4. K.M. McHugh, J.G. Eaton, G.H. Lee, H.W. Sarkas, L.H. Kidder, J.T. Snodgrass, M I.R. Manaa, and K.H. I owen, J. Chem. Phys. 91, 3792 (1989).

5. G. Ganteför, M. Gausa, K.H. Meiwes-Broer, and H.O. Lutz, Z. Phys. D 9, 253 (1989). 6. S. Yang, K.J. Taylor, M.J. Craycraft, J. Conceicao, C.L. Pettiette, O. Cheshnovsky, and R.E. Smalley, Chem. Phys. Lett. 144, 431 (1988).

7. D.W. Arnold, T.N. Kitsopoulos, and D.M. Neumark, (to be published)

8. T.N. Kitsopoulos, I.M. Waller, J.G. Loeser, and D.M. Neumark, Chem. Phys. Lett. 159 , 300 (1989).

9. G. F. Gantefor, D. M. Cox, and A. Kaldor, J. Chem. Phys. 94, 854 (1990).

10. T.N. Kitsopoulos, C.J. Chick, Y. Zhao, and D.M. Neumark, J. Chem. Phys. (1991) to appear.

11. K. Muller-Dethlefs, M. Sander, and E. W. Schlag, Z. Naturforsch. 39a, 1089 (1984).

12. J.R. Heath, A.L. Cooksy, M.H.W. Gruebele, C.A. Schmuttenmaer, and R.J. Saykally, Science 244, 564 (1989).

13. P.F. Bernath, K.H. Hinkle, and J.J, Keady, Science 244, 562 (1989).

14. N. Moazzen-Ahmadi, A.R.W. McKellar, and T. Amano, J. Chem. Phys. 91, 2140 (1989). 
15. K.S. Pitzer and E. Clementi, J. Am. Chem. Soc. 81, 4477 (1959).

16. K. Raghavachari and J. S. Binkley, J. Chem. Phys. 87, 2191 (1987).

17. G. Herzberg, Electronic Spectra of Polyatomic Molecules (Van Nostrand Reinhold, New York 1979).

18. J. A. Pople, H. B. Schlegel, R. Krishnan, D. J. DeFrees, J. S. Binkley, M. J. Frisch, R. A. Whiteside, R. F. Hout, and W. J. Herhe, Int. J. Quantum Chem. Symp. 15, 269 (1981).

19. K. Raghavachari, private communication.

20. Actually, the degeneracy of this mode is split slightly because of the $\Pi$ electronic state; the value given is the average.

21. Reference 17, p. 159.

22.P.S.H. Bolman, J.M. Brown, A. Carrington, I. Kopp, and D.A. Ramsay, Proc. R. Soc. Lond. A 343, 17 (1975).

23. J.W.C. Johns, Can. J. Phys. 39, 1738 (1961).

24. E.A. Rohlfing and J.E.M. Goldsmith, J. Opt. Soc. Am. B 7, 1915 (1990); F.J. Northrup and T.J. Sears, J. Opt. Soc. Am B 7, 1924 (1990).

25. G. Herzberg, Infrared and Raman Spectra (Van Nostrand Reinhold, New York 1979).

26. K.J. Reed, A.H. Zimmerman, H.C. Andersen, and J.I. Brauman, J. Chem. Phys. 64, $1368(1976)$

27. G. Pacchioni and J. Koutecký, J. Chem. Phys. 88, 1066 (1988).

28. L. Adar owicz, Chem. Phys. Lett. 180, 466 (1991). 

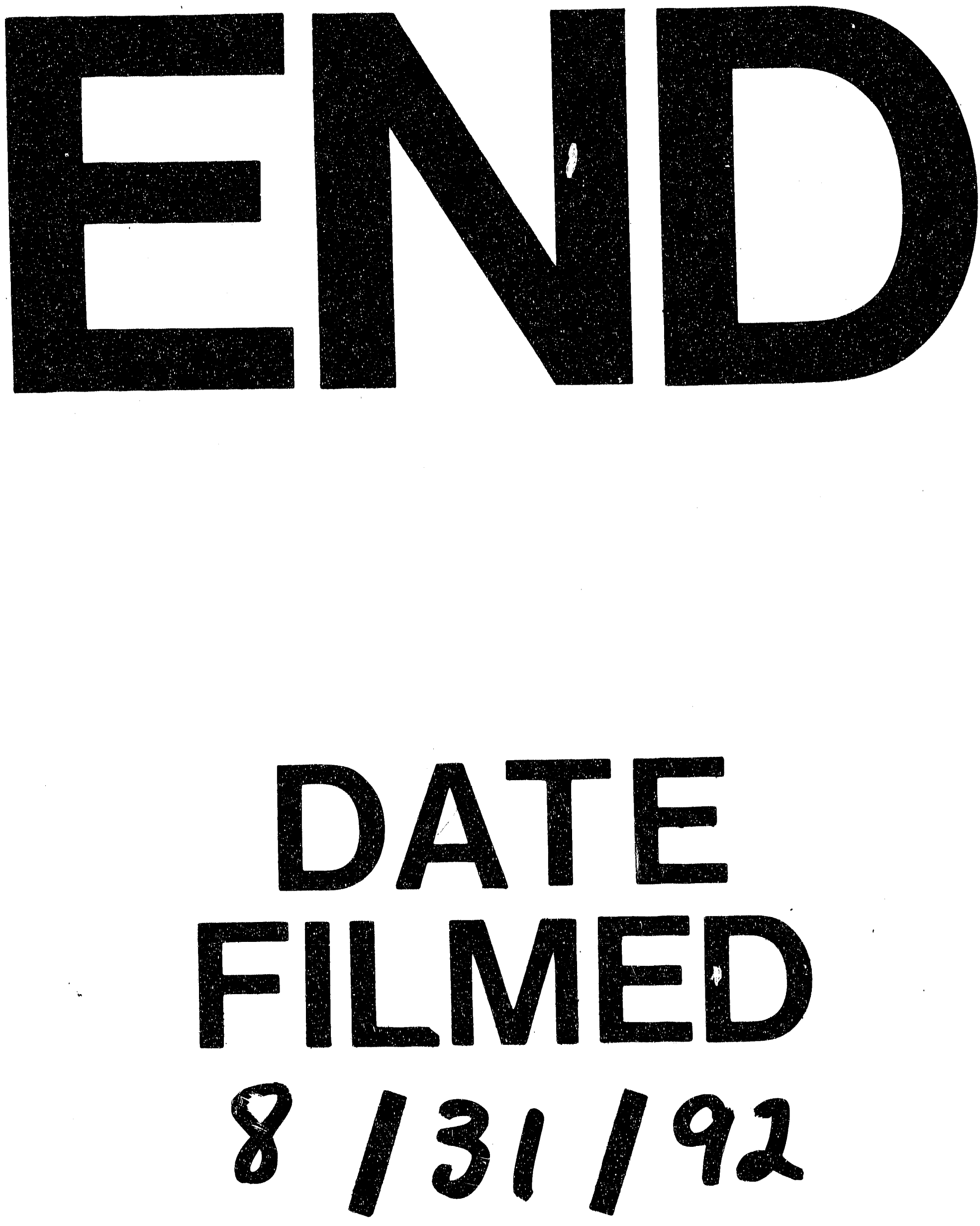

I 
